UNIVERSIDAD DE SALAMANCA

Departamento de Geología

Paleoceanografía de los sectores tropicales en los océanos Pacífico oriental y Qxlántico occidental auranke los últimos ciclos climáticos

Satsby Emperatriz Sópez Otálvaro

Jesis Dockral

Gulia, 2008 



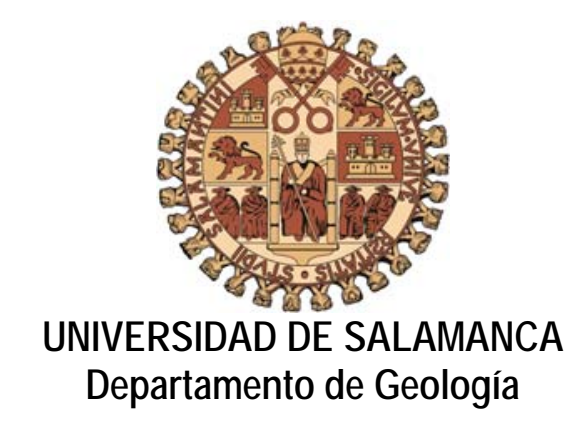

Paleoceanografía de los sectores kropicales en los océanos Pacifico oriental y Axtántico occidental aurank los ültimos ciclos climáticos

Memoria presentada por Gatsby Emperatriz Sópez Oxálvara para optar al grado de Doctor en Ciencias Seológicas por la Universidad de Salamanca

Directores de la Tesis:

Dr. Francisco Javier Sierro Sánchez

Profesor Titular del Departamento de Geología

Facultad de Ciencias

Universidad de Salamanca

Dr. José Abel Flores Villarejo

Catedrático del Departamento de Geología

Facultad de Ciencias

Universidad de Salamanca 

Dr. D. Francisco Javier Sierro Sánchez y Dr. D. José-Abel Flores Villerejo, Profesores del Área de Paleontología, en el Departamento de Geología de la Universidad de Salamanca

CERTIFICAN:

Que Dña. GATSBY EMPERATRIZ LÓPEZ OTÁLVARO ha realizado, bajo nuestra supervisión, en el Departamento de Geología de la Universidad de Salamanca el trabajo:

\title{
Paleoceanografía de los sectores tropicales en los océanos Pacífico oriental y Qulántico occidental aurank los últimos ciclos climáticos
}

La Doctoranda

Gatsby Emperatriz López Otálvaro

\author{
Los Directores
}

Dr. Francisco Javier Sierro Sánchez

Dr. José Abel Flores Villarejo 
La realización de esta tesis ha sido posible gracias a la beca de Formación de Personal Investigador (FPI) del Ministerio de Educación y Ciencia BES-2003-0010, concedida a la autora; así como a la financiación obtenida por los proyectos BTE2002-04670, CGL 2005-00642/BTE, CGL2006-10593/BTE, CGL2008-05560 del Ministerio de Educación y Ciencia, así como al Programa Consolider Ingenio “GRACCIE” (CSD2007-00067). También agradecemos a Junta de Castilla y León por el proyecto concedido al Grupo de Geociencias Oceánicas (Grupo de Excelencia GR34). Agradecemos también al Programa IODP (Integrated Ocean Drilling Program) e IMAGES por la realización de las campañas Leg 202 y PICASSO Cruise respectivamente, y por suministranos las muestras. Los datos de isótopos de oxígeno para los Testigos ODP 1240 y 1242 han sido facilitados por la Dra. Isabel Cacho y el Dr. Alan Mix respectivamente. 


\section{AGRADECIMIENTOS}

El tiempo pasa y como siempre llega el momento para todo, incluso para llevar a término esta etapa que gracias a Dios, viví con mucho gozo y entrega durante estos años de mi vida, el tiempo se me fue volando. Llegaron las personas, los lugares y los momentos del trabajo y del compartir en el tiempo de descanso o dificultad. Y aunque los sitios y las cosas dejaron huella y quizás puedan pasar, las personas estarán ahí siempre haciendo buena mella como si siempre fuera tiempo presente.

Siempre en mi memoria y con todo mi respeto, quiero nombrar a mis Profesores y Directores Dr. Francisco Javier Sierro Sánchez y Dr. José Abel Flores Villarejo, les expreso mi gratitud por el apoyo y la confianza para delegar en mi, tareas y compromisos académicos que me han permitido culminar este escalón, esencial para mi vida en todo el sentido de la palabra. Por abrirme las puertas de la Universidad de Salamanca con especial disponibilidad. Gracias por vuestra dirección, consejos y aquellas charlas amenas y sugerentes que me permitían desconectar o volver a tomar la ruta adecuada.

A la Dra. Maria Ángeles Bárcena Pernía, por sus conversaciones, las charlas y la orientación que brindó en todas las tareas de mi trabajo, y el compartir en algunos tiempos de ocio. A la ciudad de Salamanca por acogerme en medio de sus monumentos y hacerme sentir, sobretodo en las noches, en tiempos medievales ... sólo faltaron los vestidos y los carruajes.

A mi querido Profesor Dr. Luis Carlos Sánchez de Posada, Suco, por su deferencia y aquella confianza infinita que siempre me brindó desde mis lejanas tierras y desde aquellos tiempos sin aún conocerme. Y por toda aquella atención solicita por procurar mi bienestar en España. A tí junto con aquel equipo en la Secretaría del Departamento de Geología de la Universidad de Oviedo, Charo, Tina y Maria Jesús, que me brindaron su colaboración desde los mas de $10.000 \mathrm{Km}$ de distancia, siempre con diligencia y claridad para que pudiera iniciar mis estudios de Doctorado en la Universidad de Oviedo. Por supuesto, gracias infinitas también a la Universidad de Oviedo y toda su gente por aquella facilidad innata para acogerme y hacerme sentir siempre en mi casa. Al Dr. Daniel Arias Prieto, Decano de la Facultad de Geología de Oviedo, por su interés siempre diligente en permitir la experiencia profesional de los alumnos en la empresa. A mis Profesores del Departamento de Geología en Oviedo, especialmente en el área de Paleontología, Lis, Jenaro, Isabel, Montse, Susana, Miguel Arbizu por tanta amistad y tanta alegría. A Soto, mi Tutor cuando di comienzo a mi Doctorado en aquellas aulas, gracias por el cariño recibido y tu especial acogida. A Carlos Salvador y Andrés Cuesta que hicieron que 
todos los equipos informáticos y microscópicos siempre estuvieran disponibles para adelantar mi trabajo en aquellos tiempos, gracias por toda la confianza! Sin lugar a dudas gracias a Raúl Martín e Iván de la UniOvi, los ciberchicos.

Gracias de todo corazón también al Dr. Wolfgang Künt y al Dr. Hanno Kinkel en la Universidad de Kiel, a la Dra. Isabel Cacho en la Universidad de Barcelona, al Dr. Joan Grimalt en el Instituto de Química Ambiental del CSIC en Barcelona, y al Dr. Francis Groussette de la Universidad de Bourdeaux, por invitarme a sus centros científicos y por velar siempre por mi durante mis estancias. Todo siempre a la orden del día en sus laboratorios. Sin vosotros no hubiese sido posible el trabajo realizado en el corto tiempo y los muchos afanes. Al Institut für Geowissenschanften Christian-Albrechts-Universität zu Kiel, a GRC de Geociècies Marines a la Universitat de Barcelona, a I'Institut d'Investigacions Quimiques I Ambientals al CSIC a Barcelona y a la Universitat de Bourdeaux por brindarme un espacio totalmente acogedor para adelantar mi trabajo científico.

Con especial agradecimiento y deferencia me dirijo al Dr. Alan Mix de la Universidad de Oregon, al Dr. Jeremy Young del Museo de Historia Natural de Londres, al Dr. Mario Cachao de la Unviersidad de Lisboa, a la Dra. Lluisa Cros del Instituto de Ciencias del Mar en Barcelona, al Dr. Gabriel Filippelli de la Universidad de Indiana y a Nick Skinner por su amabilidad para atenderme y su interés en colaborar en mis labores científicas. Igualmente a mi amiga la Dra. Maria Angela Bassetti por su disponibilidad para orientarme en todo el sentido de la palabra, tanto a nivel académico como personal, y sobretodo, por su gran amistad, incondicional durante todos estos años pese a la distancia, gracias por tu gran apoyo! A la Dra. Sabine Becquey, con quien compartí mis primeros pasos en Salamanca y en Bourdeaux, su especial acogida, siempre y su solicitud para compartir.

Al personal de Hemeroteca y Préstamo de la Biblioteca Abraham Zacut de la Universidad de Salamanca, por tanta delicadeza en su orientación, en su servicio, en su cercanía, y por hacer propicio un especial espacio para adelantar mi labor científica.

A mis Profesores Dr. Michel Hermelin y al Dr. César Vásquez en Colombia, y a mi amigo Dr. César Mora, con aquellos consejos incansables por impulsarme y animarme con alegría en esta labor científica y de los nanofósiles calcáreos. A mi Universidad EAFIT en Medellín, que me preparó como Geóloga y me enseñó a volar, y que gracias a su eficiencia y su prestigio hicieron que la burocracia en España siempre fuera más fácil.

Al Jordi López en el CSIC en Barcelona por esa gran vocación de servicio, tanto conocimiento brindado con tanta sencillez, por la gran amistad y la facilidad que brindaba para orientarme y estar disponible siempre a pesar de la lejanía o de la hora. A la Belén Martrat, a la 
Montse Esparza y a la Patricia Alabart con tanta disponibilidad en todo momento, en sus tiempos de descanso e incluso en esta distancia entre el oriente y occidente español. Muchos kilómetros de por medio que no impidieron vuestra colaboración. $Y$ en general a todo el equipo de paleo y de salud del 321 y 306 del Instituto de Química Ambiental del CSIC y al equipo del GRC en la UB por ser tan descomplicados para brindar alegría y hacer planes, por esperarme con insistencia y paciencia hasta que pudiera salir del laboratorio para ir con vosotros, aunque eso significara esperar hasta el infinito. Y cómo no, a la ciudad de Barcelona, para todo condal.

A mis amigos de la Parroquia San Francisco en Oviedo, mis primeros amigos en España, por tantos planes juntos, por permitir que la amistad siempre se fortalezca en la distancia y porque aún seguís ahí. A los de mis queridos coros Cantabimus en Oviedo y Visboviscum en Salamanca por la amistad, las fantásticas presentaciones y los ensayos divertidos y bien trabajados. A Elena Iglesias, Julio Rodríguez y su familia, a Álvarito, a Ainhoa y su familia y todos los amigos tan especiales que encontré en el Colegio Mayor América de Oviedo. A la ciudad de Oviedo, mi primera casa en España, la que disfruté siempre, incluso sólo recordándola, una ciudad señorial y fantástica.

A mis amigos de San Martín, San Marcos y los Carmelitas, en especial al P. José que se preocupó y veló por mi estancia desde que pisé Salamanca, te agradezco inmensamente tu sentido del humor, tus consejos, las charlas amenas y tu gran disponibilidad para ayudarme en todo momento. Al P. Fernando, por estar siempre ahí, por su amistad sincera, por la cercanía regalada, por velar por mi bienestar y por soportar mis incordios. Al P. Miguel por tanta delicadeza, la chispa, las risas a todo dar, por regalarme a San Juan de la Cruz y por la dulce amistad. A las Siervas de María en Salamanca y a todos mis amigos en España y alrededor del mundo, por aquel compartir y por la amistad ferviente que en todo momento se nos regalaba. Vaya momentos!!!

Mi agradecimiento en grande para mis familiares y amigos en Colombia, los López Arango, Osorio Taborda, Granados Rojas, González Martínez, Ocampo Monsalve, Gil García, P. Iván Darío, P. Luis Eduardo, Silvio Valencia y familia, porque espiritualmente siempre estuvisteis en estas tierras conmigo, me acompañasteis y me seguisteis sin cansancio. $Y$ llena de buenos recuerdos, me dirijo a Reinel Solano, Hugo Ramos y Andrés Mantilla por la amistad a toda prueba, la cercanía, la compañía y la buena voluntad.

Con mención de honor desde siempre y para siempre a mi querida familia, la más cariñosa del mundo, en especial a ti madre que en todo momento me das rienda suelta para llevar a cabo mis propósitos, por tu orientación, tu espera incansable y tu compañía. A mi hermano, a mi padre, a mis tías y a los Dayon, ya nuestros Dayon ... A todos los he añorado 
siempre y gracias a Dios los he sentido siempre tan cerca, sin su apoyo y oraciones esta labor hubiera tomado otro rumbo. Es todo un gozo compartir con vosotros este logro que desde mucho tiempo atrás ya habíamos empezado a vivir emocionados. También pregunto a los pequeños Kiko y Valú, incondicionales y cariñosos siempre! A mi ciudad natal, Medellín, la ciudad mas hospitalaria, por siempre la ciudad de la Eterna Primavera en todo el sentido de la palabra!

Y a Tí, ya lo sabes, con quien tengo una linda historia de familia y a la vez de amistad desde siempre, y que has sido mi motor y mi freno cada vez que es necesario!

A todos aquellos que fueron testigos de este camino trazado en su día y todos los que han vivido de cerca aquellos acontecimientos y me regalaron su afecto y colaboración en el trabajo que dejo en estas páginas y también aquel trabajo que quedó por incluir en ellas. A mi país, Colombia, a quién dedico estas páginas. A todos mi más ferviente gratitud y mi admiración, no sabeis cuánto! Habéis sido una bendición para mi!!! 
¿Q ánde puedo ir lejos de $\mathcal{J}_{i}$ ? $\S_{i}$ cuando subo a la inmensidad, y cuando entro en lo pequeño, estás ahi! of. Salmo 139:8 


\section{TABLA DE CONIENIDOS}

Pág.

Agradecimientos

Resumen

i

Abstract

ix

Introducción

Objetivos

xvii

1 Variabilidad climática

$x \mathrm{xi}$

2 Los cocolitóforos

3 Área de Estudio

4 Materiales y Métodos

5 Variations in coccolithophorid production in the Eastern Equatorial Pacific at ODP Site 1240 over the last seven glacial-interglacial cycles

López-Otálvaro, G.E., Flores, J.A., Sierro, F.J., Cacho, I.

Published in Marine Micropaleontology ("in press")

doi:10.1016/j.marmicro.2007.11.009

6 Paleoproductivity and coccolith-derived carbonate contribution to sediments in the Eastern Tropical Pacific over the past $925 \mathrm{Ka}$ : ODP sites 1241 and 1242

López-Otálvaro, G.E., Flores, J.A., Sierro, F.J., Mix, A.C.

Submitted to Palaeogeography, Palaeoclimatology, Palaeoecology

7 Late Pleistocene paleoproductivity patterns during the last climatic cycle in the Guyana Basin: the calcareous nannoplankton clue

López-Otálvaro, G.E., Flores, J.A., Sierro, F.J., Cacho, I., Grimalt, J.O., Michel, E., Cortijo, E., Labeyrie, L.

Published in e-Earth ("in press")

http://www.electronic-earth-discuss.net/3/11/2008/eed-3-11-2008.html

8 Síntesis y Perspectiva Regional

9 Conclusiones

10 Apéndices 


\section{RESUMEN}

Esta Tesis Doctoral está enfocada al estudio de la variabilidad temporal y espacial de la señal de paleoproductividad y la topografía de la nutri-termoclina durante los últimos ciclos climáticos en los océanos Pacífico oriental y Atlántico occidental tropicales. Los mecanismos hidrológicos y atmosféricos que controlan estas señales están relacionados con la activación de las células de surgencia, la dinámica de las corrientes superficiales y subsuperficiales, la migración del frente ecuatorial y el desplazamiento latitudinal de la zona de convergencia intertropical ("Intertropical Convergence Zone" ITCZ).

En este estudio se analizó cuantitativamente la distribución temporal y espacial de las asociaciones de cocolitóforos y del carbonato asociado a estos organismos en las secuencias sedimentarias 1240 (estadios 15 a 1), 1241 (estadios 20 a 1) y 1242 (estadios 22 a 1), extraídas por el proyecto "Ocean Drilling Program" (ODP) 202 en el océano Pacífico oriental tropical. Adicionalmente se analizó la secuencia MD03-2616 (estadios 6 a 1) recuperada en la campaña PICASSO Cruise en el océano Atlántico occidental tropical. En términos generales, los sedimentos están compuestos principalmente por arcillas hemipelágicas con cantidades variables de nanofósiles calcáreos, foraminíferos, diatomeas y terrígenos, si bien los nanofósiles calcáreos son habitualmente el componente biogénico principal (Mix et al., 2003). Aunque algunos intervalos se caracterizan por una preservación deteriorada en los testigos 1242 y MD03-2616, tratando de enmascarando un poco el significado micropaleontológico de los perfiles de paleoproductividad y del carbonato derivado de los cocolitos, la preservación general de los ejemplares estudiados es buena a moderada, y realza el significado micropaleontológico de estos registros en todos los testigos estudiados.

La estratigrafía isotópica combinada con los datos paleomagnéticos, la bioestratrigrafía de cocolitóforos en el Pacífico oriental y de foraminíferos planctónicos en el Atlántico occidental y la estratigrafía del carbonato aportado por los cocolitos en los testigos 1241 y 1242, permitieron reconstruir el marco cronoestratigráfico de las secuencias sedimentarias estudiadas.

La estimación de la paleoproductividad superficial está basada en el cálculo del índice $\mathrm{N}$ el cual establece la relación entre los habitantes de la zona fótica superior ("small Noelaerhabdaceae, Gephyrocapsa oceanica y Emiliania huxleyi) y los habitantes de la zona fótica inferior (Florisphaera profunda). F. profunda habita la zona fótica inferior y prefiere aguas muy frías, ricas en nutrientes y con bajos niveles de luz solar, y por ello ha sido un taxón ampliamente utilizado como un indicador del desplazamiento vertical de la nutri-termoclina. Un incremento en la abundancia relativa de $F$. profunda revela la reducción en la productividad superficial debido a la profundización de la nutri-termoclina (lo cual se indica a través 
de valores del índice $\mathrm{N}$ cercanos a 0); y una reducción en su abundancia relativa indica el incremento en la producción superficial y la somerización de la nutri-termoclina (valores del índice $\mathrm{N}$ cercanos a 1). La reconstrucción de los registros del carbonato derivado de los cocolitos está basada en la densidad de la calcita, la longitud de los especímenes, una constante que depende de la forma de cada especie y el cálculo de las abundancias absolutas.

La variabilidad del perfil del carbonato derivado de los cocolitos sigue las variaciones del patrón del índice $\mathrm{N}$, lo cual indica que el perfil del carbonato derivado de los cocolitos puede ser utilizado como un nuevo indicador de paleoproductividad.

La variabilidad en la paleoproductividad y en la migración de la nutri-termoclina en el Pacífico oriental ecuatorial, no siguió un patrón glacial/interglacial durante los últimos $560 \mathrm{Ka}$, de acuerdo con el análisis de la asociación cocolitoforal. Esto sugiere que su dinámica estuvo influida por otros eventos no directamente vinculados a la escala glacial/interglacial, por ejemplo, los eventos análogos a "El Niño". Durante los períodos glaciales se registró un incremento de la paleoproductividad en la "warm pool", del Pacífico oriental tropical, se registró durante los glaciales, en los cuales la intensificación de los vientos alisios del noreste desarrollaron la surgencia costera en los golfos de Panamá, Papagayo y Tehuantepec, y evitaron la llegada masiva de aguas cálidas traídas por la contracorriente Norecuatorial desde el Pacífico occidental durante los últimos $925 \mathrm{Ka}$. Este patrón de paleoproductividad en el Pacífico oriental tropical coincide con el patrón glacial/interglacial de producción de carbonato típico en los océanos Pacífico e Índico. La máxima paleoproductividad producida por la máxima somerización de la nutritermoclina, se registró en la zona de divergencia ecuatorial del Pacífico (testigo 1240) durante los últimos $560 \mathrm{Ka}$. El registro micropaleontológico indica que la paleoproductividad fue disminuyendo hacia el norte de la cuenca de Panamá, en la "warm pool" del Pacífico oriental, indicando una profundización progresiva de la nutri-termoclina también hacia el norte de la cuenca; lo cual concuerda con la topografía actual de la nutri-termoclina en ese sector.

Adicionalmente, el análisis de la paleoproductividad en el Pacífico oriental tropical pone de manifiesto que la más alta paleoproductividad en los últimos $925 \mathrm{Ka}$, se encuentra en el período comprendido entre los estadios 22 al 6, indicando la máxima intensificación de las células de surgencia junto con el máximo desarrollo de los vientos alisios durante ese período. La mayor concentración del carbonato aportado por los cocolitos se registró durante este intervalo, y caracterizó de manera extraordinaria el evento midBrunhes (entre los estadios 15 a 8), indicando que este evento está asociado a un régimen altamente eutrófico donde la nutri-termoclina permaneció somera y facilitó una altísima paleoproductividad. Estas características oceanográficas y atmosféricas permitieron establecer una analogía con las condiciones actuales de "La Niña", y así referir los estadios 22 a 6 a un escenario similar al que causa "La Niña" hoy en día. Otra característica relevante en el mismo registro, es el descenso fuerte de la paleoproductividad 
en el estadio 5 en el Pacífico oriental ecuatorial y tropical, sugiriendo un ambiente más oligotrófico debido a un descenso drástico de la nutri-termoclina. Este escenario paleoceanográfico indica una disminución severa en la intensidad de los vientos alisios y el desplazamiento de la ITCZ hacia el ecuador. Esta relación paleoceanográfica-paleoatmosférica permite inferir condiciones análogas a "El Niño" actual durante el interglacial 5. Posteriormete, la paleoproductividad intentó recuperarse durante los estadios 4 al 1 en la zona de divergencia ecuatorial, aunque ésta no llegó a ser tan alta como lo fue durante los estadios 22 al 6, indicando de nuevo una somerización relativa de la nutri-termoclina causada por la intensificación de los vientos alisios y con ello, la afinidad a un evento análogo a "La Niña" actual. Sin embargo, en el sector de la "warm pool" en el Pacífico oriental tropical, la paleoproductividad no se incrementó durante los eventos 4 a 1, indicando que aunque los vientos alisios volvieron a intensificarse durante ese intervalo y favorecieron el aumento de la paleoproductividad en la zona de divergencia ecuatorial, éstos no fueron tan intensos como para suscitar el aumento en la paleoproductividad y la somerización de la nutri-termoclina en la "warm pool" durante ese intervalo.

En la cuenca de la Guayana, la paleoproductividad mostró cambios importantes durante el estadio 5 y de menor amplitud desde el estadio 4 hasta el Holoceno temprano, coincidiendo con la variabilidad en el patrón de insolación de verano a $65^{\circ} \mathrm{N}$. La dinámica paleoceanográfica en la cuenca de la Guayana sugiere una conexión interlatitudinal entre la insolación en el Hemisferio Norte, la ITCZ y la dinámica de los vientos alisios del sur durante el último ciclo climático. La señal micropaleontológica también permitió deducir que la paleoproductividad en la cuenca de la Guayana fue menor que en la cuenca de Panamá durante los últimos $155 \mathrm{Ka}$, indicando con ello que la nutri-termoclina ha estado más profunda en el Atlántico occidental que en el Pacífico oriental durante ese período.

De esta manera se deduce que la distribución temporal y espacial de los cocolitóforos estuvo estrechamente vinculada a la disponibilidad de nutrientes en el océano superficial como consecuencia de la intensificación de las células de surgencia durante el Pleistoceno medio y tardío en los océanos Pacífico oriental y Atlántico occidental tropicales. No obstante, no se descarta la influencia del aporte continental de nutrientes en la cuenca de la Guayana, si bien la asociación de cocolitóforos (autóctona y alóctona) y la ausencia de otros organismos como fitolitolitos y diatomeas de agua dulce, no sugieren la influencia de los flujos de los ríos Orinoco y Amazonas que descargan sus sedimentos en el testigo MD03-2616. 


\section{ABSTRACT}

This $\mathrm{PhD}$ Thesis is focused on the study of the temporal and spatial variability of the paleoproductivity record, the nutri-thermocline topography and all the mechanisms associated to them in the Eastern Tropical Pacific (ETP) and the Western Tropical Atlantic (WTA) over the last climatic cycles. These mechanisms are related to the strengthening of the upwelling cells, the dynamic of the surface and subsurface currents, the migration of the Equatorial Front and the latitudinal displacement of the Intertropical Convergence Zone (ITCZ). The purpose was carried out through the analysis of the calcareous nannoplankton assemblage along the sedimentary sequences from the Ocean Drilling Program (ODP) Leg 202, sites 1240 (Marine Isotope Events MIS 15 to 1), 1241 (MIS 20 to 1) and 1242 (MIS 22 to 1) recovered from the ETP. The calcareous nannoplankton assemblage from core MD03-2616 was also studied for paleoceanographical purposes along MIS 6 to 1. This last sequence was recovered by the PICASSO Cruise in the WTA. Sediments mostly consist of hemipelagic clays with variable amounts of calcareous nannofossils, foraminifers, diatoms and terrigenous matter, being the calcareous nannofossils the primary biogenic component (Mix et al., 2003). Although several intervals are characterized by deteriorated carbonate preservation at sites 1242 and MD03-2616, masking the micropaleontological signal, the overall preservational pattern varies between good and moderate, enhancing the micropaleontological significance of all the records studied.

The isotope stratigraphy, the Brunhes/Matuyama datum, the biostratigraphy of coccolithophores in the ETP, the biostratigraphy of planktonic foraminifers in the Western Tropical Atlantic (WTA) and the coccolith-derived carbonate stratigraphy at sites 1241 and 1242, allowed us to reconstruct the chronostratigraphic framework in all the sedimentary sequences studied.

Paleoproductivity is based on the $\mathrm{N}$ ratio estimations. This index establishes the relationship between the upper ("small Noelaerhabdaceae, Gephyrocapsa oceanica y Emiliania huxleyi) and lower (Florisphaera profunda) photic zone species. F. profunda inhabits the lower photic zone and prefers cooler and nutrient-rich waters with low light levels. In consequence its relative abundance can be used as a proxy for the vertical displacement of the nutri-thermocline. Increases in the relative abundance of $F$. profunda suggest lower surface productivity due to the deepening of the nutri-thermocline ( $\mathrm{N}$ ratio values close to 0 ). Decreases in its relative abundance indicate increasing productivities due to shoaling of the nutri-thermocline ( $\mathrm{N}$ ratio values close to 
1). The reconstruction of the coccolith-derived carbonate profiles is based upon the calcite density, the species-specific mean length, the shape constant and the absolute abundances. The variability of the coccolith carbonate rate (CAR) parallels that of the $\mathrm{N}$ ratio, suggesting a close relationship between paleoproductivity and the carbonate production. Therefore, we used the CAR record as a new proxy of paleoproductivity.

According to the analysis of the calcareous nannoplankton assemblage, the paleoproductivity variation and nutri-thermocline displacements in the Eastern Equatorial Pacific (EEP) do not follow a glacial/interglacial pattern over the past $560 \mathrm{Ka}$ (MIS 15 to 1). This suggests that the system was influenced by other events different to those related to the glacial/interglacial scale, such as the El Niño-like events. Paleoproductivity increased in ETP warm pool, during the glacial episodes, following a paleoproductivity pattern recorded in other regions of the Pacific and Indian oceans. Those events were characterized by the strengthening of the northeast Trade winds which favored the coastal upwelling in the Panama, Papagayo and Tehuantepec gulfs and avoid the arrival of the warm water regime of the North Equatorial Countercurrent (NECC) in the ETP over the past $925 \mathrm{Ka}$. The micropaleontological signal suggests that the maximum paleoproductivity and shallower nutri-thermocline occurred in the EEP (Site 1240) and indicates a northward deepening of the nutri-thermocline in the Panama basin with the lowest productivity values in the same direction (Site 1242), in agreement with modern oceanographic conditions in that region.

The paleoproductivity analysis in the ETP also suggests the highest paleoproductivity was recorded during MIS 22 to 6 (925 Ka to $128 \mathrm{Ka}$ ) when maximum strength of the Trade winds favored the intensification of the upwelling cells. The highest coccolith-derived carbonate concentration was recorded during that interval, characterizing the mid-Brunhes Event (MIS 15 to 8) and hence, suggesting that this period was related to a highly eutrophic setting. These oceanographic and atmospheric dynamic during MIS 22 to 6 allowed to establish a similarity with recent conditions prevailing during La Niña events. In addition, the paleoproductivity records suggest a strong deepening of the nutri-thermocline during MIS 5 in the ETP, which is linked to a more oligotrophic setting. That oceanographic setting is driven by a severe weakening of the Trade winds and the southward displacement of the ITCZ towards the equator, and hence, showing conditions similar to those prevailing today during EI Niño events. In contrast, during MIS 4 to 1 a relative increase in paleoproductivity linked to a shoaling of the nutri-thermocline, indicate the onset of the an eutrophic regime in the EEP caused by stronger Trade winds. This setting 
was related with La Niña-like events. However, paleoproductivity did not increase in the "warm pool" during the same interval (MIS 4 to 1), indicating that the Trade winds were not strong enough to allow a northward extension of the eutrophic regime in the ETP.

The paleoproductivity pattern in the Guyana Basin underwent stronger changes during MIS 5 compared to MIS 4 and the early Holocene and these changes coincide with insolation variations at $65^{\circ} \mathrm{N}$. The paleoceanographic dynamic in the Guayana Basin suggests an interlatitudinal connection between the Northern Hemisphere insolation, the ITCZ and the Southeast Trade wind dynamic during the last climatic cycle. The micropaleontological record also allowed us to infer that paleoproductivity was lower in the Guyana Basin than in the Panama Basin during the past $155 \mathrm{Ka}$, indicating that the nutri-thermocline was deeper in the WTA than in the ETP.

We thus can infer that the temporal and spatial distribution of the calcareous nannoplancton was closely linked to the availability of nutrients in the surface ocean in response to the intensification of the upwelling cells during the middle and late Pleistocene in the ETP and WTA. Although the calcareous nannoplankton assemblage (authoctonous and alloctonous) and the lack of phytoliths and fresh water diatoms did not suggest the influence of the Orinoco and Amazon rivers, the input of continental-derived nutrients to the WTA cannot be discarded. 


\section{INTRODUCCIÓN}

El estudio de la forma de las cuencas marinas, de las características de las corrientes oceánicas que empujan grandes masas de agua superficiales y profundas distribuyendo el calor interlatitudinalmente, y de la productividad biológica, son algunos de los objetivos de la ciencia que estudia la historia del océano: la Paleoceanografía. Esta ciencia está estrechamente relacionada con la dinámica atmosférica (que controla la intensidad y dirección de las corrientes marinas), y con el ciclo hidrológico del continente que arrastra sedimentos, vegetación y compuestos orgánicos e inorgánicos terrestres hacia el océano. La relación océano-atmósfera-continente se manifiesta a través de muchos indicadores de tipo micropaleontológico, sedimentológico, y geoquímico que han sido almacenados en el registro sedimentario a través del tiempo geológico (Wefer et al., 1999).

Así como la composición y la distribución de las comunidades planctónicas actuales en el océano global dependen de las condiciones físicas y biológicas de su hábitat; la composición florística de los sedimentos extraídos de los fondos oceánicos está relacionada con las condiciones paleoceanográficas, las cuales dependen directamente de la variabilidad climática (Andruleit et al., 2005).

Hoy en día el estudio de la productividad marina ha sido el centro de atención de muchas investigaciones paleoceanográficas. La productividad marina está limitada por la disponibilidad de nutrientes que llega través de los ríos o de la surgencia de aguas intermedias. La productividad marina regula el $\mathrm{CO}_{2}$ atmosférico y por tanto el clima global mediante la transferencia de $\mathrm{CO}_{2}$ atmosférico hacia el océano profundo (Philander, 1995). El océano tropical revela la más alta diversidad de la vida marina y por ello ofrece un interés especial desde el punto de vista paleoceanográfico. A ello se le agrega el papel relevante del trópico en la variabilidad climática de la Tierra (cf. Funnell et al., 1996).

La distribución temporal de los foraminíferos planctónicos en el océano Atlántico occidental tropical también ha cambiado en función de la variabilidad climática, tal como lo han puesto de manifiesto diversos autores (Ericson y Wollin, 1956; Kennett y Huddlestun, 1972; Prell y Damuth, 1978; entre otros) al menos durante el Cuaternario tardío. La composición de la asociación de esta comunidad zooplanctónica ha permitido correlacionar la estratigrafía secuencial de las Zonas Climáticas de Ericson a lo largo de ese sector del Atlántico (Ericson y Wollin, 1956). Dicha estratigrafía está basada en la aparición (durante los interglaciales) y desaparición temporal (durante los glaciales) del grupo de Globorotalia menardii (Ericson y Wollin, 1956) y de la especie Pulleniatina obliquiloculata (Kennett y Huddlestun, 1972) facilitando la afinación del modelo de edad elaborado a partir de la estratigrafía isotópica del $\delta^{18} \mathrm{O}$ en el testigo MD03-2616. 
Esta Tesis Doctoral se centra en el estudio de la paleoproductividad de los cocolitóforos y del carbonato aportado por estos organismos como un nuevo indicador de paleoproductividad, con la finalidad de reconstruir la evolución paleoceanográfica del océano superficial en los sectores tropicales del Pacífico oriental y Atlántico occidental durante el Pleistoceno medio y tardío.

El Pacífico oriental tropical es un área de alta productividad, responsable del $50 \%$ de la nueva producción primaria global (Chavez y Barber, 1987), controlada no sólo por la variabilidad estacional de sus características hidrológicas y atmosféricas, sino también por el impacto de la variabilidad interanual del fenómeno de "El Niño-Oscilación del Sur" ("El Niño Southern Oscillation", ENSO; Philander, 1995). E impacto del fenómeno de ENSO repercute en la variabilidad interanual de la temperatura de la Tierra y en los patrones de precipitación (Ropelewski y Halpert, 1987). Por ende, es una región de gran significado en el estudio del océano y del clima en el pasado. El Atlántico occidental es una región que además de las variaciones estacionales del océano y de la atmósfera, ha sido afectado por importantes descargas continentales de los ríos más importantes del mundo, Amazonas y Orinoco (Milliman y Meade, 1983) y además constituye una de las principales rutas de la transferencia de calor de las masas de agua superficiales, intermedias y profundas entre el Atlántico Norte y Sur (Rühlemann et al., 2001).

Siguiendo la estructura del manuscrito, el capítulo 1 hace referencia a la variabilidad climática en el Pleistoceno a escala orbital, milenaria e interanual, el fenómeno de ENSO y una revisión de los eventos característicos durante el último millón de años (Pleistoceno medio y tardío) en el océano global. Las referencias del Capítulo 1 también incluyen aquellas correspondientes a la Introducción de esta Tesis.

El capítulo 2 concierne a los cocolitóforos, la definición de los términos comúnmente utilizados, la clasificación, algunas consideraciones biológicas, biogeográficas, químicas y su utilidad en la investigación paleoceanográfica y paleoecológica.

El capítulo 3 describe las zonas de estudio dentro de los marcos geológico-tectónico y paleoceanográfico, mientras que el capítulo 4 corresponde a la descripción del material utilizado y los métodos de obtención de datos y de resultados.

Los capítulos 5,6 y 7 se refieren a los resultados y discusiones previamente publicados (o en fase de publicaión) como artículos científicos o enviados a revistas internacionales de reconocido prestigio, en los cuales la doctoranda es la autora principal. El capítulo 5 describe la evolución de la productividad y su relación con la migración de la nutri-termoclina y la dinámica atmosférica en el Pacífico oriental ecuatorial a lo largo de los últimos $560 \mathrm{Ka}$. Adicionalmente, el capítulo reseña con especial énfasis la sensibilidad de la asociación florística a los eventos "mid-Brunhes" y estadio 5, y a los eventos análogos a "El Niño" a lo 
largo de la secuencia sedimentaria estudiada. El capítulo 6 explica la influencia de la paleoproductividad y de la disolución del carbonato en el patrón glacial/interglacial del registro del carbonato aportado por los cocolitóforos en la "warm pool" del Pacífico oriental tropical, a través de los últimos $925 \mathrm{Ka}$. La señal micropaleontológica y la dinámica de la nutri-termoclina dan un soporte adicional a la teoría de la variabilidad glacial/interglacial de la producción del carbonato tradicionalmente observada en los océanos Pacífico e Índico; y permiten observar la sensibilidad de la asociación cocolitoforal al Evento "midBrunhes" y a los eventos análogos a "El Niño" durante los pasados 925 Ka en ese sector del Pacífico. El capítulo 7 expone los cambios de la paleoproductividad y de la dinámica de la nutri-termoclina durante el último ciclo climático en la cuenca de la Guayana; la relación de está con la dinámica de los vientos alisios y de la zona de convergencia intertropical ("Intertropical Convergence Zone", ITCZ) y con la variabilidad de la insolación de verano del Hemisferio Norte.

En el capítulo 8 presentamos una síntesis de los resultados y discusiones de los artículos cientíicos contenidos en los capítulos 5, 6 y 7 y las líneas de investigación futuras. Establece una comparación interlatitudinal e inter-cuenca de los sectores tropicales estudiados enfocada en la topografía de la nutritermoclina y en los cambios de la productividad durante el último ciclo climático. Adicionalmente se incluye una síntesis sobre las consideraciones paleoecológicas de los principales taxones identificados en este estudio. Este capitulo resume el aporte científico de esta Tesis Doctoral a los objetivos científicos planteados por las campañas oceanográficas a las cuales pertenece el material estudiado ("Ocean Drilling Program" ODP 202 y PICASSO). Por último, el capítulo 9 presenta las conclusiones más relevantes de esta Tesis Doctoral.

Los apéndices incluyen el listado de los taxones estudiados en esta investigación (Apéndice A), las notas taxonómicas y las diferencias morfológicas usadas para al género Gephyrocapsa (Apéndice B), las fotografías de los taxones de interés realizadas con microscopio electrónico y petrográfico (Apéndice $\mathrm{C}$ ), y el índice de los acrónimos utilizados (Apéndice D). 


\section{OBJ EIIVOS}

El objetivo general que enmarca la realización de esta Tesis Doctoral consiste en explicar la forma en la que los sectores tropicales del Pacífico oriental y Atlántico occidental, han respondido a las perturbaciones del clima. Los registros sedimentarios obtenidos están caracterizados por una alta tasa de sedimentación, lo cual ha permitido disponer de series resolutivas que han puesto en marcha una serie de objetivos a escala orbital (decenas a centenas de miles de años).

Objetivos particulares:

1. Establecer un modelo biocronológico en los sedimentos del Pleistoceno medio y tardío en los sectores tropicales del Pacífico oriental y Atlántico occidental:

a. Definir los biohorizontes de cocolitóforos a partir de su estudio cuantitativo en el océano Pacífico oriental tropical, apoyados en la estratigrafía isotópica $\delta^{18} O$ y los datos paleomagnéticos aportados por otros grupos científicos.

b. Establecer la Zonación Climática del océano Atlántico occidental en la cuenca de la Guayana a partir del análisis cualitativo de los foraminíferos planctónicos en el testigo MD03-2616.

C. Obtener un modelo de edad para el testigo MD03-2616 en el Atlántico occidental basado en la estratigrafía isotópica $\delta^{18} 0$ del foraminífero bentónico Uvigerina peregrina, la Zonación Climática calibrada para la cuenca de la Guayana y la bioestratigrafía de los cocolitóforos en el mismo testigo.

2. Estudiar la evolución de la dinámica atmosférico-hidrográfica del océano superficial en relación con la productividad de los cocolitofóros, la contribución de su carbonato aportado al sedimento oceánico (y por ende, su participación e importancia en el ciclo del $\mathrm{CO}_{2}$ ) y la migración vertical de la nutri-termoclina en los sectores tropicales del Pacífico oriental y Atlántico occidental a lo largo del Pleistoceno medio y tardío. 
a. Estimar los flujos (tasa de acumulación) de las especies de cocolitóforos identificadas y la masa de carbonato aportada al océano profundo mediante la agrupación de especies por rangos biométricos, como nueva temática en los estudios paleoecológicos.

b. Evaluar la preservación del carbonato de cocolitos y foraminíferos mediante el empleo de técnicas cualitativas como la observación directa de los individuos a través de los microscopios petrográfico, electrónico y estereoscópico, y del índice CEX' de cocolitos.

c. Identificar los patrones de variabilidad climática que han controlado la producción de los cocolitóforos y de su carbonato aportado, y la migración vertical de la nutri-termoclina a lo largo del Pleistoceno medio y tardío, haciendo especial énfasis en el Evento "mid-Brunhes" y el estadio isotópico 5.

d. Determinar la influencia de perturbaciones climáticas análogas a aquellas que son producidas a escalas de tiempo menores que las orbitales como lo es "El Niño - la Oscilación del Sur" (ENSO).

3. Elaborar una interpretación sintética de los resultados obtenidos y una comparación interlatitudinal de la evolución del océano superficial en las cuencas de Panamá y de la Guayana. 


\section{Variabilidad climática}

1.1. Introducción

1.2. El registro climático en los sedimentos marinos: los isótopos de oxígeno

1.3. La escala Orbital: La teoría de Milankovitch

1.4. La escala milenaria

1.5. La escala decadal: "El Niño - la Oscilación del Sur"

1.6. Variabilidad climática durante el Cuaternario

1.7. Eventos análogos al fenómeno de "El Niño" durante el Pleistoceno

Referencias 


\subsection{Introducción}

El sistema climático terrestre ha sido controlado por diversos mecanismos que han actuado a diferentes escalas del tiempo geológico: los procesos tectónicos, los cambios orbitales de la Tierra, cambios en la radiación del sol, variaciones en la dinámica oceánica, etc.

Los procesos tectónicos se generan por el calor en el interior de la Tierra y afectan la geografía de la superficie terrestre. Ellos se manifiestan a través del movimiento de las placas tectónicas, el levantamiento de las cordilleras y la apertura y cierre de las cuencas oceánicas. Su intervalo de tiempo comprende un rango de varios millones de años. Es la escala de tiempo más larga en la variabilidad climática de la Tierra. Los cambios orbitales terrestres se producen como consecuencia de cambios periódicos en el sistema solar. Estos cambios afectan a la radiación que la Tierra recibe del Sol en el tiempo y el espacio. Los parámetros orbitales cambian entre decenas a cientos de miles de años. La variación de la radiación solar también ocasiona cambios en la cantidad de radiación que llega a la Tierra, y se establece en escalas decadales, seculares y milenarias, correspondiendo a las escalas más cortas en la variabilidad climática. La investigación realizada en esta tesis doctoral concierne a la escala temporal orbital y milenaria, sobre las cuales se hará una breve reseña explicativa.

\subsection{E registro climático en los sedimentos marinos: los isótopos de oxígeno}

Las primeras aplicaciones de los isótopos estables del oxígeno sobre esqueletos calcáreos fueron realizadas con foraminíferos fósiles en sedimentos marinos y los resultados obtenidos permitieron correlacionar la composición isotópica con la profundidad en que las diferentes especies habitaban (Emiliani, 1954; Van Donk, 1977 y Bé, 1977).

Aunque los fundamentos de la termometría isotópica se basaban en las variaciones del cociente 180/160 en función de la temperatura a la que precipitaba el carbonato cálcico (Urey, 1947), posteriormente se observó que los cambios de estado y las variaciones de salinidad del agua introducian variaciones isotópicas mucho mayores, en otras palabras el fraccionamiento isotópico era significativamente superior que el estrictamente debido a la temperatura de 
calcificación. Estas diferencias son debidas a que las moléculas de agua con isótopos ligeros $\left({ }^{16} \mathrm{O} \circ \mathrm{H}\right)$ tienen una mayor presión de vapor (volatilidad) que las constituidas con isótopos pesados ( ${ }^{18} 0$ o D -deuterio). Así, durante el proceso de condensación, se extrae el isótopo pesado y el agua restante que no se condensa se enriquece más en el isótopo ligero. Las últimas fases de condensación se producen a temperaturas más frías, las cuales se alcanzan a altas latitudes (en los polos), produciendo mínimos valores isotópicos respecto a aquellos alcanzados a bajas latitudes.

Los fenómenos de condensación y evaporación solo alteran la composición isotópica de la capa superficial del océano temporalmente pues las aguas continentales tardan poco tiempo en volver al mar. Durante los episodios glaciales, que duraron varios miles de años, la prolongada permanencia de las aguas continentales en forma de hielo, pobres en los isótopos pesados, alteró la composición isotópica del agua de todos los océanos de la tierra (Criss, 1999) (Fig. 1.1)

La notación delta (ס; Craig 1953) se utiliza debido a la poca abundancia relativa de los isótopos pesados (del 1\% para el carbono hasta el $0.01 \%$ en el oxígeno y deuterio).

$$
\delta^{18} O=\left[\frac{\left({ }^{18} \mathrm{O} /{ }^{16} \mathrm{O}\right) \mathrm{m}}{\left({ }^{18} \mathrm{O} /{ }_{16} \mathrm{O}\right) \mathrm{ref}}-1\right] * 1000
$$

Los valores se expresan en tanto por mil para que los valores sean más comprensibles, donde "m" corresponde a la muestra y "ref" a un patron de referencia, que actualmente es el valor isotópico medio del agua de mar (tomado como cero en la escala delta). En trabajos antiguos y algunos recientes se utiliza como referencia el PDB ("PeeDee Belemnite").

Basándose en este principio de fraccionamiento de los isótopos del agua, las aguas superficiales del océano se enriquecen más en los isótopos pesados cuando las tasas de evaporación son más altas y el efecto contrario tiene lugar por las aportaciones fluviales de aguas continentales (Broecker y Van Donk, 1970).

Las series de tiempo del registro de $\delta^{18} O$ de las conchas de los foraminíferos han generado un registro importante de los cambios del clima. El $\delta^{18} \mathrm{O}$ en los foraminíferos está en función de la 
temperatura y del $\delta^{18} O$ del agua en el cual se forma. El $\delta^{18} O$ del agua del mar es una función del volumen de hielo global y de la salinidad del agua (Lisiecki y Raymo, 2005).

a
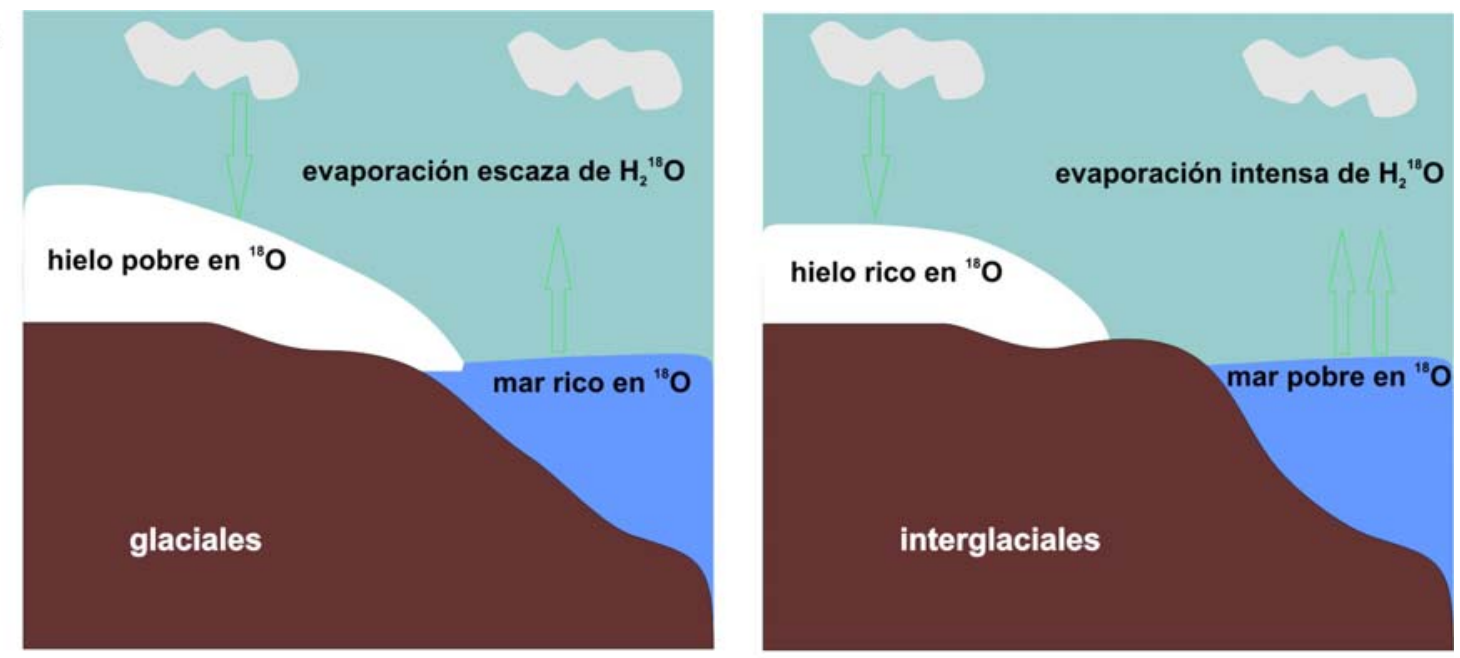

b

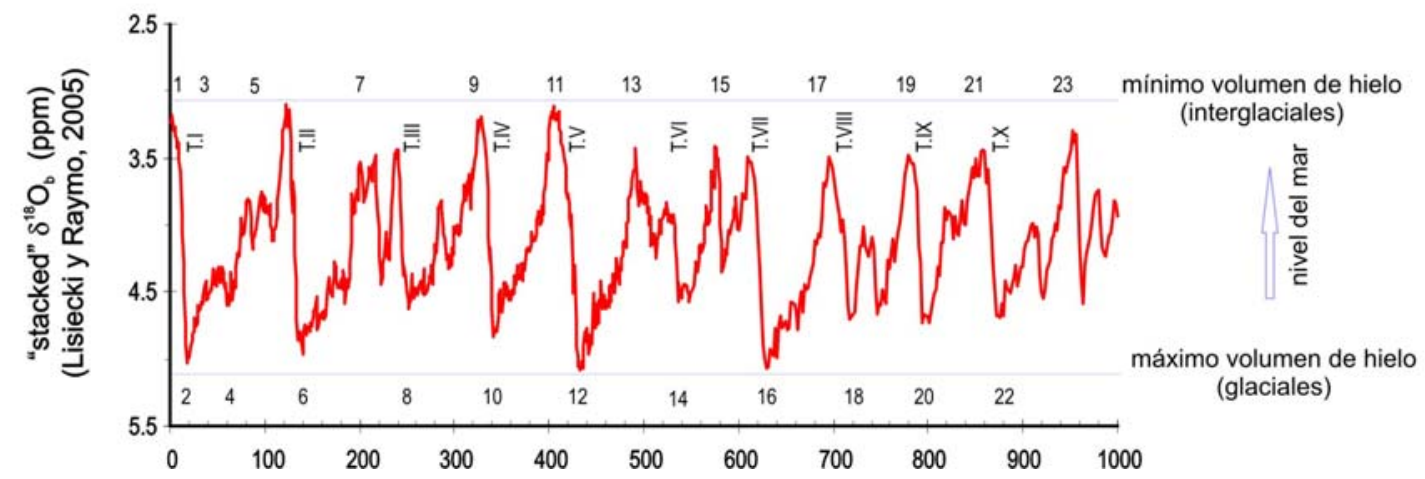

Fig. 1.1. a) Fraccionamiento isotópico de los isótopos de oxígeno durante los períodos glaciales e interglaciales. Adoptada de Uriarte-Cantolla (2003). b) Señal isotópica $\delta^{18} 0$ "stack" durante los eventos glaciales (altos valores de $\delta^{18} \mathrm{O}$ ) e interglaciales (bajos valores de $\delta^{18} \mathrm{O}$ ). Los números impares sobre el registro del $\delta^{18} \mathrm{O}$ corresponden a los eventos glaciales, los números pares corresponden a los eventos interglaciales, y los números romanos corresponden a las terminaciones (T) durante el último millón de años (1 Ma=1000 Ka) (Lisiecki y Raymo, 2005).

Los registros isotópicos han sido muy conocidos por sus variaciones de gran amplitud y las fluctuaciones rápidas de la temperatura del océano, la cual se ha reconstruido a partir de ellos (Shackleton et al., 2004).

La señal isotópica del $\delta^{18} 0$ indica que durante el proceso glacial/interglacial (expansión/fusión del hielo), un aumento gradual en los valores isotópicos indica una progresiva acumulación de hielo en los polos y/o un enfriamiento gradual que culmina en un máximo glacial (máximo volumen de hielo). Ese máximo isotópico y de volumen de hielo es seguido por un descenso isotópico brusco que identifica una deglaciación rápida o calentamiento (terminaciones) que da paso a un período interglacial (Kennett, 1982) (Fig. 1.1). 
Debido a la similitud observada en los registros marinos del $\delta^{18} O$ y en la naturaleza global de la señal del volumen de hielo, las medidas del $\delta^{18} 0$ también son utilizadas para situar el registro climático marino en una escala de tiempo común. Los registros "stack" son perfiles en los que se ha hecho un promedio de los registros de $\delta^{18} \mathrm{O}$ de varios testigos con la finalidad de mejorar la relación señal/ruido del registro climático, calibrar las características isotópicas para facilitar la comparación y definir un marco cronológico estándar. La influencia de la variabilidad local y regional puede perturbar en cierta medida la señal del registro climático. Por ello, los registros bentónicos del $\delta^{18} \mathrm{O}$ producen una mejor señal isotópica que la de los planctónicos porque el océano profundo es más uniforme en temperatura y salinidad que el agua superficial (Lisiecki y Raymo, 2005).

La serie isotópica "stack" más ampliamente usada ha sido la construida por el SPECMAP ("Mapping Spectral Variability in Global Climate Project"; Imbrie et al., 1984) a partir de 5 registros de foraminíferos planctónicos. Este registro se extiende sobre los últimos $\sim 750 \mathrm{Ka}$. Algunos estudios apoyan la estructura básica del SPECMAP, sin embargo en la actualidad, se dispone de registros de $\delta^{18} \mathrm{O}$ más largos en el tiempo y de más amplia resolución (Shackleton et al., 1990; Pisias et al., 1990; Raymo, 1997; Huybers y Wunsch, 2004, Lisiecki y Raymo, 2005; entre otros). El perfil LR04 de Lisiecki y Raymo (2005) contiene 57 registros bentónicos calibrados según la técnica de correlación gráfica y se extiende sobre los últimos $5 \mathrm{Ma}$ a muy alta resolución. EI LR04 apoya el SPECMAP sobre los últimos $625 \mathrm{Ka}$; es la primera curva que se extiende más allá de los $2.5 \mathrm{Ma}$, y ha podido definir 24 estadios isotópicos adicionales en el Plioceno inferior.

Esas variaciones en el volumen del hielo y en la temperatura identificadas en la estratigrafía isotópica han permitido estudiar las variaciones climáticas en la Tierra documentadas por los ciclos Milankovitch, las cuales han sido examinadas en los estudios de los sedimentos marinos (Emiliani y Shackleton, 1974; Imbrie et al., 1993).

\subsection{La escala Orbital: La teońa de Milankovitch}

La teoría orbital fue mencionada inicialmente por el matemático francés Joseph Adhemar en 1842, pero no tuvo acogida, luego el geólogo escocés James Croll la retomó, y por último Milutin Milankovitch la impulsó como teoría. Sin embargo, no fue aceptada hasta que Hays et al (1976) 
la aplicó a las variaciones de temperatura registradas en sedimentos marinos de aguas profundas de edades que comprenden los últimos $450 \mathrm{Ka}$. Estos autores identificaron series cíclicas en la señal de temperatura, las cuales de manera paralela a la variación orbital, indicaban el retroceso y el avance del volumen del hielo sobre inmensas superficies. Actualmente se han señalado algunas evidencias en las que se constata que los gases invernadero $\left(\mathrm{CO}_{2}\right.$, vapor de agua y metano), han desempeñado un papel importante en la amplificación de los efectos orbitales, por ello la asociación de la periodicidad de $100 \mathrm{Ka}$ en el clima aún no es del todo clara.

Las alteraciones de la órbita terrestre debidas a la atracción gravitatoria ejercida por otros planetas, se reflejan como cambios en la distribución e intensidad de la radiación solar que incide sobre la Tierra, causando variaciones en el clima a diversas escalas temporales que van desde de miles a cientos de miles de años. Milutin Milankovitch, astrofísico serbio (1879-1958) calculó la radiación solar recibida por la Tierra de acuerdo con las estaciones y la latitud, y su influencia sobre la expansión y la retracción de los hielos. Milankovitch calculó las variaciones temporales de la excentricidad, la oblicuidad y la precesión (Maslin et al., 2001) (Fig. 1.2). De los tres parámetros orbitales descritos por Milankovitch la excentricidad es el único que afecta a la insolación neta anual sobre el planeta, los otros dos solo afectan a la distribución latitudinal y estacional de la radiación.

La excentricidad (e): es un parámetro que define la desviación de la órbita de la tierra alrededor del sol, respecto a un círculo, se calcula mediante el cociente entre la distancia del centro a uno de los focos (c) y el semieje mayor de la elipse (a) e=c/a. Una órbita más excéntrica presenta valores próximos a 1, mientras que una órbita circular presenta valores e=0 (Paillard, 2001; Fig. 1.2a, 1.2d). Los cambios en la excentricidad influyen en la variación de la distancia que separa la Tierra del sol, y con ello el calor recibido por la Tierra. Los ciclos de excentricidad se producen aproximadamente cada 100 y $400 \mathrm{Ka}$. Cuando la órbita es más excéntrica, la radiación solar que recibe la Tierra es mayor en el perihelio (entre un 20\% a 30\%) que en el afelio (cuando la Tierra está más alejada del sol; Lutgens y Tarbuck, 1995; Maslin et al., 2001).

La oblicuidad $(\varepsilon)$ es la inclinación del eje de rotación de la tierra respecto el plano de la eclíptica (plano que contiene la órbita elíptica alrededor del sol; Fig. 1.2). En la actualidad toma un valor de $23.44^{\circ}$, oscila entre $21.8^{\circ}$ y $24.4^{\circ}$ y tiene una periodicidad próxima a los $41 \mathrm{Ka}$. La oblicuidad 
es la responsable de la estacionalidad del clima terrestre y la variación que experimenta tiene su mayor impacto sobre la insolación de las regiones polares (Maslin et al., 2001).

El eje rotacional de la Tierra describe un círculo alrededor de la vertical sobre la eclíptica, y ésto es lo que se conoce como la precesión de los equinoccios, la cual puede asemejarse a los giros de un trompo alrededor de la vertical. La precesión se produce por el momento de fuerza ejercido por la atracción gravitatoria combinada de los objetos que forman el sistema solar. La precesión tiene dos componentes, la precesión axial y la precesión elíptica. La precesión axial ocurre debido al torque (momento de fuerza) establecido entre sol y los planetas en el área ecuatorial, causando la rotación del eje. La precesión elíptica ocurre cuando la órbita elíptica cambia y con ella la fecha del perihelio. La combinación de estos parámetros da lugar a ciclicidades de 19-23 Ka. La unidad de estos dos componentes hace que el periheliio coincida con la estación de verano en cada hemisferio aproximadamente cada $21.7 \mathrm{Ka}$, dando lugar a la precesión de los equinoccios (Maslin et al., 2001).

Los equinoccios corresponden a las épocas del año en las que el eje de rotación de la tierra se encuentra perpendicular respecto a la dirección del sol y coinciden con el inicio de las estaciones de primavera y otoño alternativamente en cada hemisferio, durante esta época ambos hemisferios reciben la misma cantidad de energía del sol. Los solsticios, o inicio de las estaciones invernal y estival, tienen lugar cuando el eje de rotación está en la misma dirección del sol, durante esta época el hemisferio que apunta hacia el sol, recibe más radiación que el que se encuentra en dirección opuesta. El efecto de la precesión es el continuo avance de la fecha de los equinoccios en el calendario en cada año sideral sucesivo. Si la tierra describiera una órbita circular alrededor del sol la precesión no tendría efecto sobre el clima. Tratándose de una órbita elíptica, la distancia al sol y por tanto la radiación recibida, varían a lo largo de todo el recorrido, tomando los valores mínimos durante el afelio y máximos durante el perihelio. Así pues, la precesión hace que las diferentes estaciones del año reciban diferente radiacion solar en función de la fase del ciclo de precesión en que se encuentren. En la actualidad, en el hemisferio norte (sur), el equinoccio de primavera (otoño) ocurre el 20 de marzo $\left(\lambda=0^{\circ}\right.$, longitud solar, posición en la órbita de traslación), el solsticio de verano (invierno) el 21 de Junio $\left(\lambda=90^{\circ}\right)$, el equinoccio de otoño (primavera) el 22 de setiembre $\left(\lambda=180^{\circ}\right)$ y el solsticio de invierno (verano) el 21 de diciembre $\left(\lambda=270^{\circ}\right)$ (Fig. 1.2d). Actualmente, la Tierra pasa por el perihelio a principios de enero, mientras que pasa por el afelio a principios de julio. 

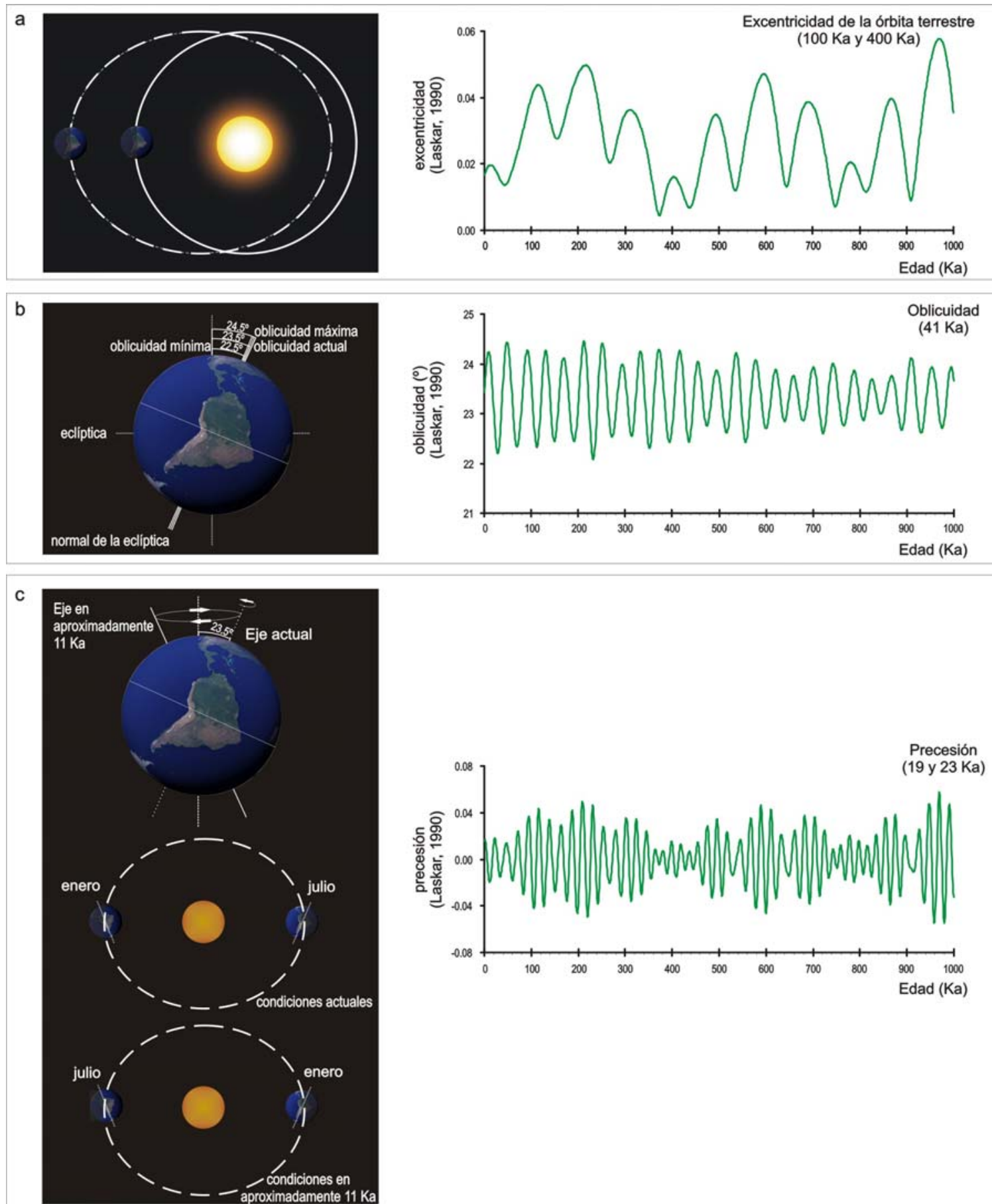

Fig. 1.2. Variables astronómicas que han influido en los cambios de la órbita de la Tierra. A la izquierda los parámetros orbitales: a) Variación de la excentricidad de la Tierra, desde una órbita más excéntrica (línea punteada) a una menos excéntrica (línea continua). b) Oblicuidad (inclinación del eje de la Tierra) actualmente y en aproximadamente $11 \mathrm{Ka} .24 .5^{\circ}$ y $22.5^{\circ}$ representan los valores máximo y mínimo de variación. c) Precesión de la Tierra en la actualidad y en $11 \mathrm{Ka}$ aproximadamente. Las figuras de la izquierda fueron adoptadas de Nolan Atkins (http://apollo.Isc.vsc.edu/classes/met130/). A la derecha, el registro de cada variable a lo largo del último 1 Ma según el método Laskar (1990) calculado a través del programa Analyseries 1.1 (Paillard et al., 1996). 


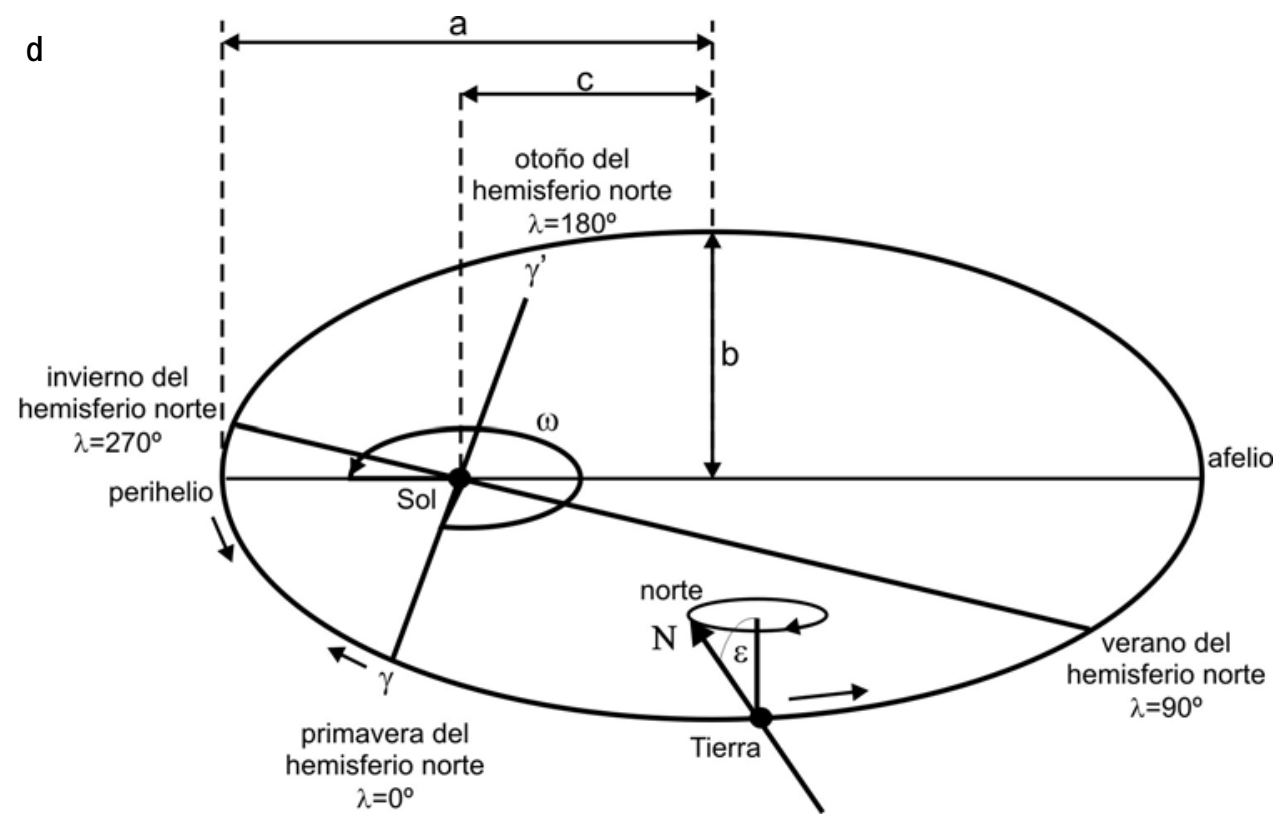

Fig. 1.2. Variables astronómicas que han influido en los cambios de la órbita de la Tierra (continuación). d) La excentricidad (e) está definida como e=c/a, donde a es el semieje mayor y c es la distancia entre el foco y el centro de la elipse. El semieje menor $b$ está definido por el teorema de Pitágoras $\left(a^{2}=b^{2}+c^{2}\right)$. El valor de la excentricidad actual es e $=0.0167$, indicando que la orbita actual de la Tierra es muy cercana a la forma circular. El ángulo actual de la oblicuidad es $\varepsilon=23.44^{\circ}$, indicando el ángulo que se forma en la intersección del plano ecuatorial de la Tierra con el plano de la eclíptica: la intersección está definida por la línea $\gamma-\gamma^{\prime}$ y la posición de los equinoccios y solsticios. La cofiguración actual hace que la Tierra esté más cercana al Sol (perihelio) $\sim 3$ de enero, unas pocas semanas después del comienzo del invierno en el hemisferio norte (o solsticio de invierno, $\lambda=270^{\circ}, 21$ de diciembre). Esta posición, relativa a la primavera del hemisferio norte (o equinoccio vernal $\gamma, \lambda=0^{\circ}$ ) está medida por el ángulo $\omega . \gamma$, tiene un período de $25.8 \mathrm{Ka}$, mientras que el perihelio varía con períodos de $100 \mathrm{Ka}$ ó $400 \mathrm{Ka}$. Así, las variaciones del $\gamma$ y del perihelio generan un período de precesión que varía entre $23 \mathrm{Ka}$ y $19 \mathrm{Ka}$. Actualmente, el equinoccio vernal $\left(\lambda=0^{\circ}\right)$ es el 20 de marzo, el solsticio del hemisferio norte $\left(\lambda=90^{\circ}\right)$, es el 21 de junio; el equinoccio de otoño $\left(\lambda=180^{\circ}\right)$ es el 22 de septiembre; y el solsticio de invierno $\left(\lambda=270^{\circ}\right)$ el 21 de diciembre. Estas fechas están asociadas a la variación de la radiación solar que llega a la Tierra causada especialmente por los cambios en la excentricidad y en el ángulo $\omega$. Modificada de Paillard (2001).

En estas condiciones y atendiendo sólo a la radiación recibida del sol, los inviernos boreales deberían ser más suaves que los australes y los veranos australes más calurosos que los boreales (Paillard, 2001). El parámetro de la precesión está acoplado directamente con la excentricidad, manifestándose esta última en la modulación de amplitud de la precesión que se puede apreciar claramente como una ondulación envolvente con una frecuencia próxima a los $100 \mathrm{Ka}$.

La combinación de los efectos de excentricidad, oblicuidad y precesión permite realizar el cálculo de insolación para cualquier latitud en el tiempo. La máxima variación en la radiación solar experimentada en la Tierra durante los últimos $600 \mathrm{Ka}$, es equivalente a la diferencia de la cantidad de radiación del verano recibida a los $65^{\circ} \mathrm{N}$ y la recibida a los $77^{\circ} \mathrm{N}$. Sin embargo, cada parámetro orbital tiene un efecto diferente según la latitud (Maslin et al., 2001). 


\subsection{La escala milenaria}

Los primeros registros de la última glaciación obtenidos a través de los testigos de hielo, demostraron que hubo una intensa variabilidad a escala milenaria, caracterizada por eventos cálidos abruptos (interestadiales) que duraron desde cientos a miles de años. Estas oscilaciones se conocen como oscilaciones Dansgaard/Oeschger (D-O) y fueron descritos por primera vez en los testigos de hielo de Groenlandia. (Dansgaard et al., 1993). También se han observado inversiones frías en la cuenca de Santa Bárbara (Weaver, 1999), en el Pacífico nororiental (Lund y Mix, 1998) y en el Atlántico tropical (Hughen et al. 1996). El último evento corresponde al Younger Dryas, el cual ocurrió entre 12.7 Ka y 11.65 Ka BP (Dansgaard et al., 1989) (Fig. 1.3).

Broecker et al. (1990) propusieron un mecanismo para explicar la variabilidad D-O. Este mecanismo está basado en los principios fisico-químicos de las masas de agua y son explicados a través de una cinta transportadora de sal y calor en el océano global, la Circulación Termohalina ("Termohaline Circulation" THC, o "Atlantic Meridional Overturning Circulation", AMOC). Los principales elementos de la densidad de las aguas hacen referencia a su temperatura y salinidad; cualquier cambio en ellas debido a las interacciones del océanoatmósfera, o debidas a la circulación oceánica misma, influirán en el comportamiento de la Circulación Termohalina (Warren, 1981). En el Atlántico, el flujo inter-hemisférico de las corrientes superficiales transporta aguas cálidas y relativamente salinas desde el trópico hacia el norte. Durante este trayecto las aguas superficiales desprenden calor a la atmósfera, aumentando la temperatura de los vientos que se dirigen hacia Europa. Cuando estas aguas superficiales llegan al Atlántico norte el agua ha incrementado su salinidad y se ha enfriado lo suficiente como para adquirir mayor densidad y hundirse en el océano profundo, dando lugar a la formación del Agua Noratlántica Profunda ("North Atlantic Deep Water", NADW) que luego fluye hacia la Antártida, como una cinta transportadora (Maslin et al., 2001) (Fig. 1.3).

En el Océano del Sur el Agua Antártica de Fondo ("Antartic Bottom Water", AABW) se forma en las costas "polinias", áreas donde los vientos que soplan del Antártico llevan el mar helado lejos de la ribera continental exponiendo las aguas superficiales a menor temperatura. Esto hace que se genere más hielo y el agua se torne más salada y por tanto más densa y pueda hundirse. De esta manera, se producen las aguas más frías y más saladas del océano. El agua que se hunde en la Antártida fluye hacia el norte y aflora en los océanos Pacífico e Índico donde no hay 


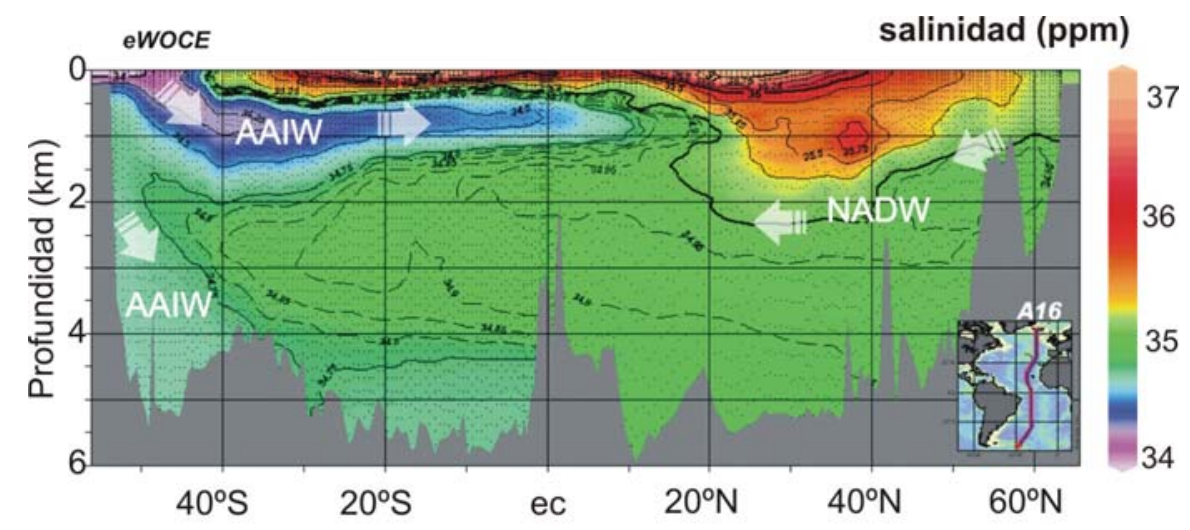

b

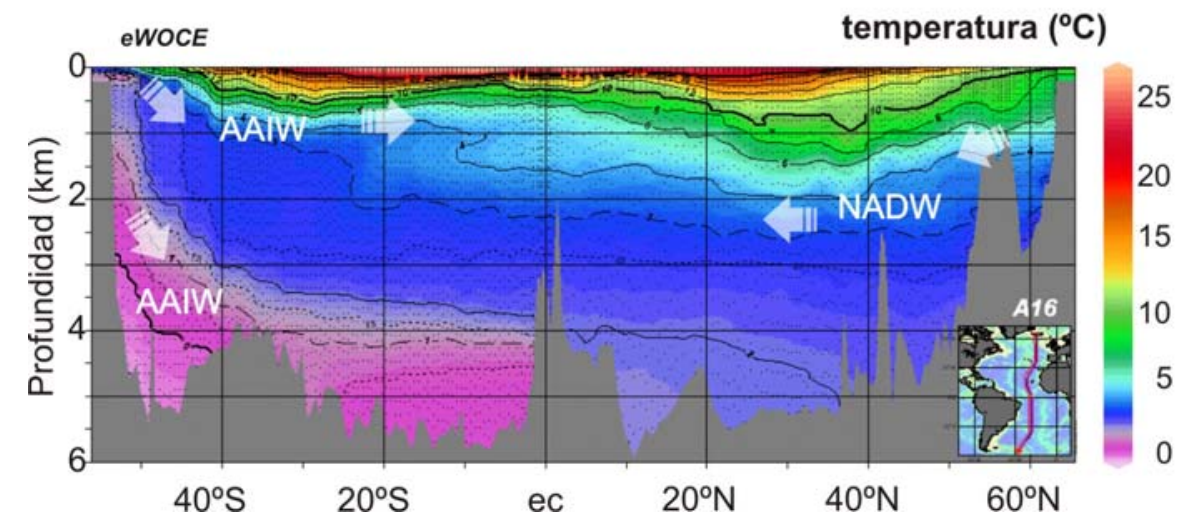

C

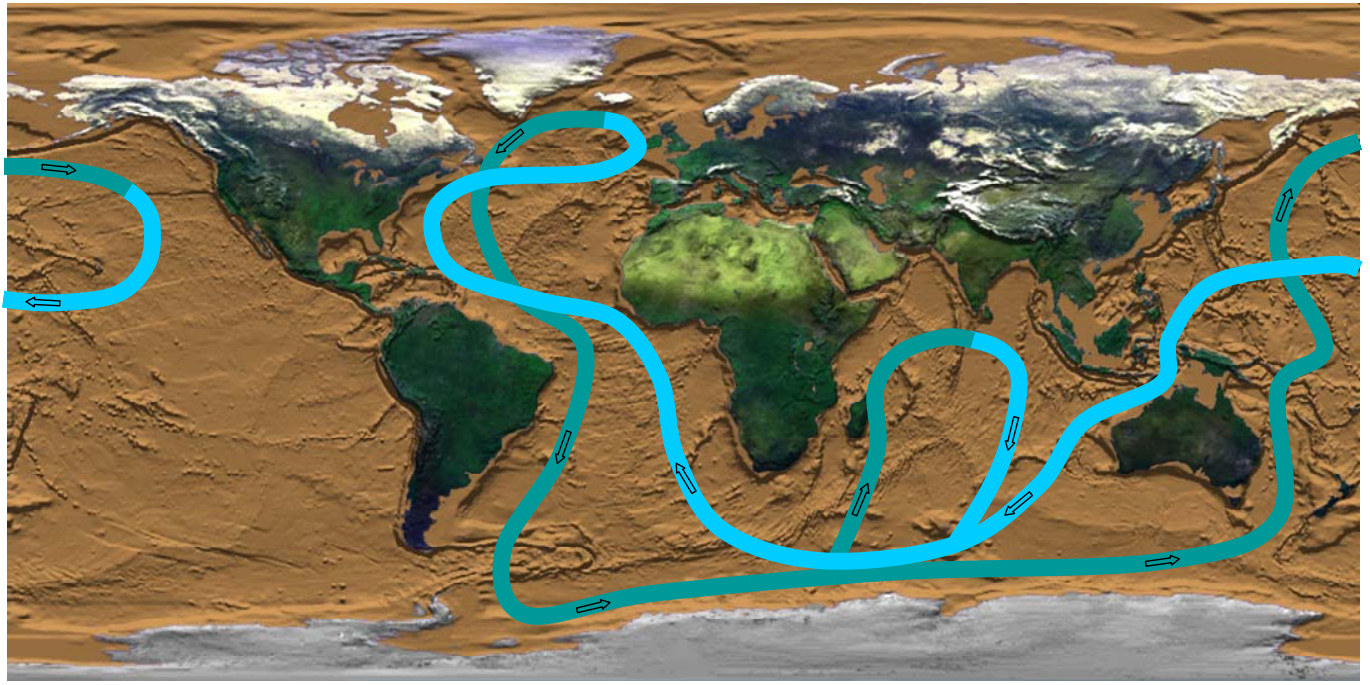

Fig. 1.3. a) y b): Corte interlatitudinal que muestra el hundimiento de las masas de agua y la formación de agua profunda en el océano Atlántico norte y sur, y algunas de sus propiedades fisico-químicas (temperatura y salinidad) en el transecto paleoceanográfico meridional A16 tomada del eWOCE (World Ocean Circulation Experiment) (Schlitzer, 2000). AAIW, Agua Antarctica Intermedia; NADW, Agua Profunda Noratlántica; AABW, Agua Antartica de Fondo. c) La circulación Termohalina. En el Atlántico Norte, las aguas superficiales relativamente más cálidas (cinta azul) se enfrían y se hunden para comenzar el proceso termohalino. El hundimiento de aguas frías y salinas en el Atántico Norte permite la producción del Agua Profunda Noratlántica (NADW, cinta verde), la cual fluye a través del océano Atlántico hacia el sur, transporta calor a través de los océanos Pacífico Norte, Índico y Atlántico en dirección norte. El recorrido de la circulación Termohalina fue adoptado de Bröecker y Denton (1989). Mapa de fondo de la figura 1c muestra la topografía del fondo del océano en falso color y fue tomado de http://visibleearth.nasa.gov. 
hundimiento de aguas. También fluye hacia el Atlántico, pero el flujo de la NADW es más intensa en ese sector (Burroughs, 2001).

Cuando esta cinta transportadora se interrumpe, cesa la formación de la NADW. Se han elaborado dos hipótesis o modelos para explicar este proceso. La primera por medio del desplazamiento del frente polar hacia menores latitudes, haciendo que las corrientes del sur se debiliten. Este mecanismo produce aguas muy frías pero poco densas impidiendo que el agua se hunda, como ocurrió durante el último glacial. La segunda se establece a través de la llegada de agua dulce, a través de la fusión de los casquetes de hielo, la descarga de los ríos o el aumento en la precipitación (Maslin et al., 2001). Durante la pausa de la cinta transportadora los vientos dominantes transportan aire frío hacia Europa. Este frío permanecerá hasta que las aguas del hemisferio sur se vuelvan tan saladas que predominen sobre las aguas más dulces del norte, fluyan violentamente y activen nuevamente la cinta transportadora (Alley, 2006). Así, la AABW fluye desde la Antártida hacia el Atlántico norte debajo de la NADW. De esta manera cuando la NADW para o disminuye su velocidad, la AABW se acelera y afecta el transporte de calor en la dirección opuesta (Maslin et al., 2001).

La THC se refiere entonces a la circulación oceánica a gran escala que es controlada por los gradientes de densidad, creados a partir de cambios en la temperatura y en el contenido de sal. Las corrientes superficiales controladas por el viento, llevan el calor del Atlántico ecuatorial hacia los polos favoreciendo la producción de la NADW (Fig. 1.3).

Heinrich (1988) observó la presencia de 6 intervalos con concentraciones anómalas de fragmentos líticos durante la última glaciación. Como esos detritos venían del continente (propiamente de Canadá, Bond et al., 1992), él concluyó que los líticos manifestaban 6 intervalos en los que aumentó el volumen de casquetes de hielo en el Atlántico Norte. Cada ciclo Heinrich estuvo conformado por dos fases. La primera corresponde al crecimiento del casquete de Laurentia en el continente y la segunda a su fusión, la cual le permitió expandirse hacia el estrecho de Hudson (McAyeal, 1993). Los eventos Heinrich revelaron una ciclicidad de $10 \mathrm{Ka}$, y aparecieron entre cada ciclo D-O (Bond et al., 1993; Broecker, 1994) (Fig. 1.4).

Bond et al. (1993) observaron que las oscilaciones D-O siguieron un patrón de "diente de sierra" ("saw-tooth pattern"), llamado ciclo Bond, el cual contiene oscilaciones D-O sucesivas que incluyen interestadiales que se van haciendo progresivamente más fríos. Cada ciclo Bond 
finaliza con un evento Heinrich, después del cual sucedió un calentamiento rápido haciendo comenzar nuevamente el proceso.

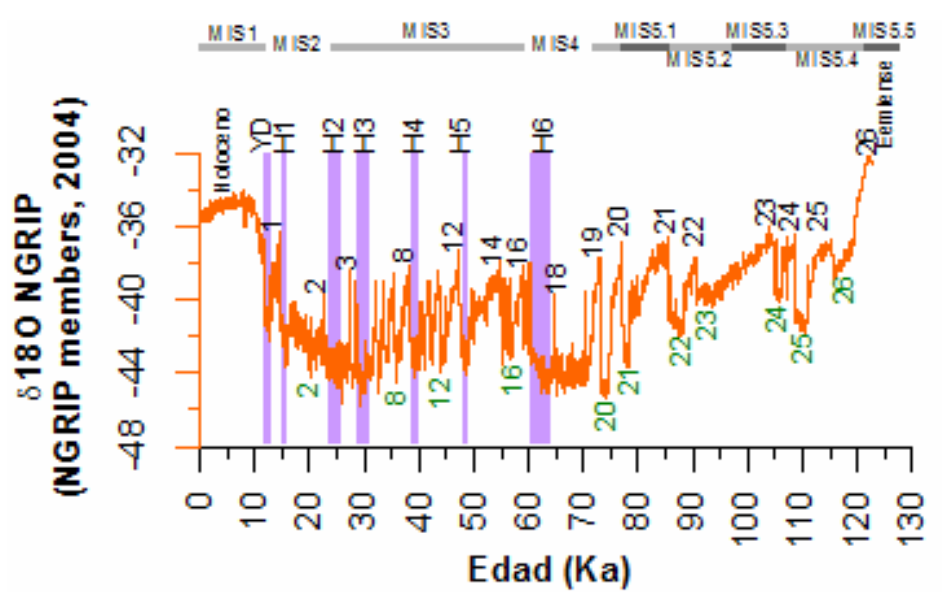

Fig. 1.4. Perfil del $\delta^{18} \mathrm{O}$ de las burbujas de aire del testigo de hielo NGRIP localizado en Groenlandia (NGRIP members, 2004). Los números en color negro al pie de la curva indican algunos interestadiales (eventos cálidos). Los números en color verde indican algunos estadiales (eventos fríos). YD: Evento Younger Dryas. H: Evento Heinrich (numerados del 1 al 6). MIS: "Marine Isotopic Stage", estadio isotópico marino. MIS par: evento glacial, MIS impar: evento interglacial.

\subsection{La escala decadal: "E Niño- la Oscilación del Sur"}

Los pescadores peruanos fueron los primeros en relatar un fenómeno que tenía lugar cada pocos años en torno a la Navidad y que consistía en la desaparición de la pesca en una zona habitualmente rica, junto con el estancamiento de aguas anormalmente cálidas. Ese fenómeno fué bautizado como "El Niño" por su proximidad a tan señalada festividad. Los eventos de "El Niño" están asociados no sólo a cambios anómalos en los patrones climáticos en el Pacífico oriental, también se asocia a cambios poco frecuentes alrededor del mundo: genera desastrosas consecuencias para la pesquería y provoca inundaciones catastróficas en la costa pacífica de Suramérica, mientras que Australia e Indonesia se ven afectadas por intensas sequías (Rasmusson, 1985); la frecuencia de huracanes aumenta en el Pacífico y disminuye en el Atlántico (Thurman \& Burton, 2001); al mismo tiempo las aves y la vida marina tradicionales desaparecen temporalmente (Philander, 1995).

Alternativamente, tenía lugar el descenso de las temperaturas superficiales por debajo del promedio en las aguas del Pacífico oriental, marcando el comienzo de la fase opuesta y complementaria a "El Niño", por lo que fué llamada la fase de "La Niña". En la fase de "La Niña", 
las diferencias en la presión superficial a lo largo del Pacífico Tropical se incrementan por encima del promedio (Philander, 1995).

La dinámica océano-atmósfera en el Pacífico tropical esta conducida por la célula de circulación de la Oscilación del Sur, llamada Circulación Walker (Fig. 1.5). La Circulación Walker afecta los vientos alisios surorientales que convergen en la célula de baja presión de Indonesia y Australia, se levantan y producen altas tasas de precipitación y altas temperaturas superficiales en el océano $\left(29^{\circ} \mathrm{C}-30^{\circ} \mathrm{C}\right)$ en el área de bajas presiones. Como consecuencia de esta dinámica, la termoclina en el Pacífico occidental se desarrolla hacia los $100 \mathrm{~m}$ de profundidad, mientras que en el Pacífico oriental la termoclina se hace somera $(\sim 30 \mathrm{~m})$ y proporciona temperaturas superficiales del agua entre $\operatorname{los} 22^{\circ} \mathrm{C}$ a $24^{\circ} \mathrm{C}$. Las diferencias de la temperatura superficial de las aguas y de la profundidad de la termoclina a ambos lados del Pacífico tropical resultan de la dinámica de los vientos alisios que proporcionan la surgencia de aguas en el Pacífico oriental y arrastran las aguas superficiales hacia el Pacífico occidental. El aire seco desciende sobre el área de altas presiones de Suramérica, y genera altas tasas de evaporación y un clima árido sobre la región (Fig. 1.5).

A raíz de las investigaciones de Gilbert Walker sobre el comportamiento de los monzones, se pudo precisar que había una serie de anomalías que estaba alterando la dinámica normal océano-atmósfera a lo largo del Pacífico tropical. Sus observaciones constataron que las intensas sequías durante el sistema de bajas presiones en la India y Australia eran coincidentes con escenarios inusuales de intensa precipitación relacionados con el sistema de altas presiones en el Pacífico tropical, y demostró la existencia de una fluctuación interanual irregular que llamó Oscilación del Sur, la cual involucraba cambios en los patrones de lluvias y vientos sobre los océanos Pacífico tropical e Índico (Philander, 1995). Posteriormente un grupo de oceanógrafos liderados por Bjerknes durante los años 60's, pudieron obtener datos suficientes para concluir que las anomalías de las temperaturas superficiales del océano en el Pacífico tropical se asociaban a la Oscilación del Sur, y empezaron a ser referidas como eventos "El Niño- la Oscilación del Sur" (“El Niño Southern Oscillation”, ENSO). Los eventos ENSO consisten de dos fases, una muy cálida asociada a "El Niño" que además precede a la fase muy fría asociada a "La Niña". Sus frecuencias se superponen a las variaciones estacionales en los patrones que rigen el océano y la atmósfera (Philander, 1995). El fenómeno ENSO está centrado a lo largo del ecuador y ocurre irregularmente a escala interanual (del orden de 1 a 10 años) (Philander, 1995; Wang y Fiedler, 2006) (Fig. 1.5). 


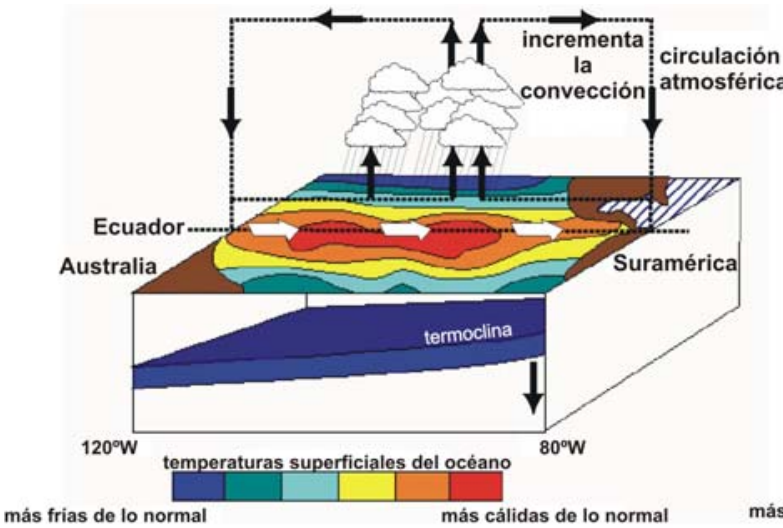

Condiciones EI Niño

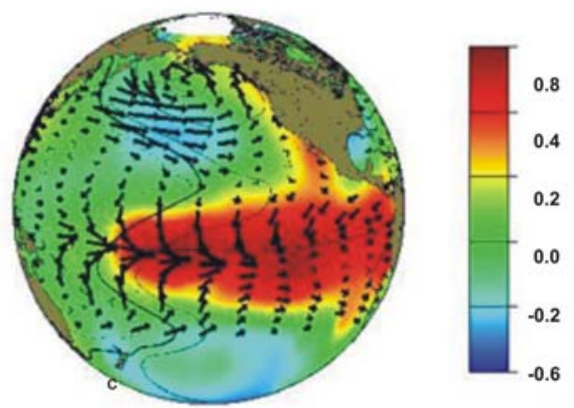

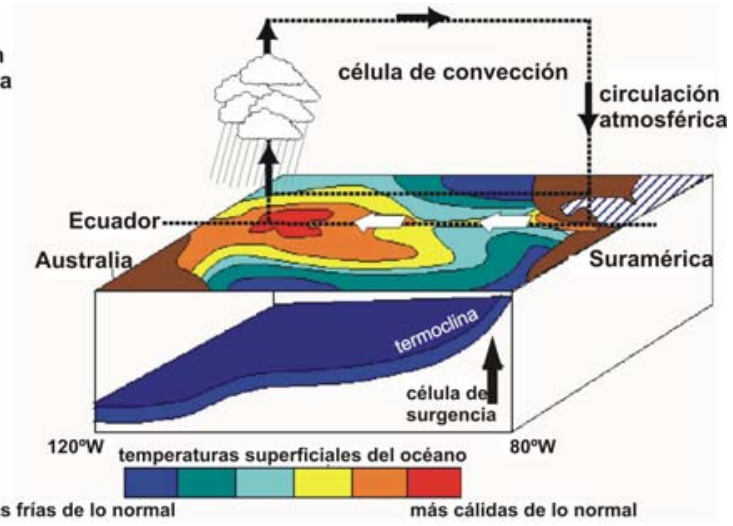

b Condiciones La Niña

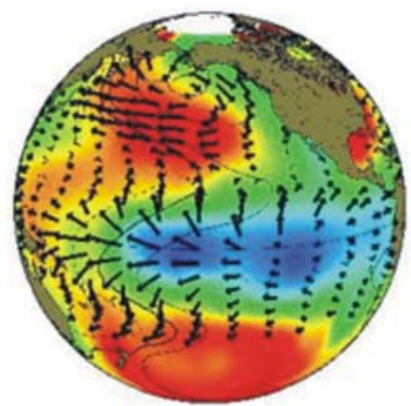

Fig. 1.5. "El Niño - Oscilación del Sur". a) Fase cálida de los eventos ENSO. La célula de convección se desplaza desde el Pacífico occidental hacia el oriental y con ella la masa de agua cálida superficial, incrementando las temperaturas y profundizando la termoclina en el Pacífico oriental. b) Fase fría de los eventos ENSO. La célula de convección se desplaza hacia el Pacífico occidental y con ella la masa de agua cálida, somerizando la termoclina en el Pacífico oriental. c) La escala de colores corresponde a las anomalías de temperatura. Durante la fase cálida de "El Niño", el régimen de los vientos alisios se debilitan y con ellos las corrientes superficiales. Durante la fase fría de "La Niña", los alisios se hacen más intensos de lo normal e intensifican notablemente las corrientes que arrastran toda el agua cálida superficial hacia el Pacífico occidental. Los vectores indican la dirección y magnitud de la intensidad de los vientos. (Las figuras fueron tomadas de Mantua, 2000 y http://www.weathersa.co.za/References/elnino.jsp)

Pocos meses antes de la llegada de "El Niño", la interacción océano-atmósfera en el trópico cambia. La célula de baja presión comienza a desplazarse hacia el este entre Octubre y Noviembre, abriendo el camino a la llegada de "El Niño". Asociado a ello están el debilitamiento de los alisios, el colapso de la surgencia de aguas y la profundización de la termoclina en el Pacífico oriental, generando una capa gruesa de aguas cálidas en esa región (Fig. 1.5). Los vientos que provienen del oeste ("westerlies") se tornan más intensos, aceleran las contracorrientes en el Pacífico y promueven el desarrollo de las ondas Kelvin que suprimen la surgencia de aguas en el Pacífico oriental. La intensificación del flujo de la contracorriente Norecuatorial (que fluye desde el Pacífico occidental hacia el oriental), genera el levantamiento del nivel del mar al occidente de América, y luego se dirige hacia las altas latitudes en ambos 
hemisferios. Durante esta fase las condiciones océano-atmósfera en el Pacífico occidental se invierten con respecto al Pacífico oriental (Wyrtki, 1975), y en este escenario las diferencias en la presión superficial a través del Pacífico tropical se hacen pequeñas.

Los eventos de "El Niño" y "La Niña" tienden a desarrollarse cada vez de diferente manera. Muchos modelos océano-atmósfera han revelado que existe una variedad de modos de oscilación. Algunos están relacionados con una fase de propagación que se dirige hacia el este y vientos del oeste dominantes (como fue observado durante "El Niño" en 1982); y otros son estacionales (Philander, 1995). Las propiedades de un modo, como su dirección de propagación por ejemplo, dependen de los procesos que determinan las variaciones en la temperatura superficial del océano. Por ejemplo, si los cambios en la tasa de surgencia de agua fría dominan la variación de las temperaturas superficiales, la fase de propagación de las anomalías se dirige hacia el este. Si predomina la advección horizontal, la dirección de propagación depende del signo de los gradientes de temperatura superficial, y pueden ser hacia el oeste.

El Atlántico experimenta un fenómeno muy similar a "El Niño", en el que la precipitación es muy intensa sobre la costa árida del suroeste de África. "El Niño" en el Océano Pacífico está asociado principalmente al movimiento de la zona de convergencia atmosférica hacia el este, mientras que el fenómeno en el Atlántico está asociado al desplazamiento hacia el sur de la zona de convergencia de los vientos alisios, llamada Zona de Convergencia Intertropical ("Intertropical Convergence Zone", ITCZ) (Philander, 1995).

\subsection{Variabilidad climática durante el Cuatemario}

El inicio del Cuaternario está datado en $1.8 \mathrm{Ma}$, siendo ligeramente más joven que el techo del evento paleomagnético normal Olduvai. Las evidencias paleontológicas y de isótopos de oxígeno ponen de manifiesto que el Cuaternario representa un período caracterizado por glaciaciones más severas y extensas que aquellas ocurridas durante el Terciario, particularmente acontecidas sobre los continentes localizados en el Hemisferio Norte, pero también sobre la Antártida. Sin embargo, el Cuaternario representa cambios en la localización geográfica de los límites entre las masas de agua (Kennett, 1982), pero no la creación de nuevas condiciones paleoceanográficas, paleoclimáticas o paleobiogeográficas como durante el límite Eoceno-Oligoceno o el Mioceno medio y tardío. 
El Cuaternario cubre dos intervalos, el Pleistoceno y el Holoceno. El Pleistoceno se extiende entre los últimos $1.6 \mathrm{Ma}$ hasta $10 \mathrm{Ka}$ y está caracterizado por repetidas glaciaciones. Está dividido en el Pleistoceno temprano (1.8 Ma hasta $780 \mathrm{Ka}$ ), Pleistoceno medio (780 Ka hasta 128 $\mathrm{Ka}$ ) y Pleistoceno tardío (128 Ka hasta $10 \mathrm{Ka}$ ). El Holoceno cubre los últimos $10 \mathrm{Ka}$. El clima durante el último millón de años ha sido dominado por ciclos de glaciación con períodos cercanos a los $23 \mathrm{Ka}, 41 \mathrm{Ka}$ y $100 \mathrm{Ka}$. Siguiendo la Teoría de Milankovitch, los dos ciclos más pequeños pueden explicarse como respuestas a los ciclos de insolación controlados por la precesión y la oblicuidad (Imbrie et al., 1993). Más del 90\% del último millón de años se ha caracterizado por extensas áreas terrestres cubiertas por hielo y el predominio de temperaturas muy frías en el océano y en la atmósfera. Este régimen glacial ha sido interrumpido por intervalos de relativo calentamiento, por ejemplo los estadios $11,9,5$ e (este último también llamado Eemiense en el continente) y el Holoceno. En resumen, el Cuaternario ha sido marcado por el desarrollo y la fusión de los casquetes de hielo en el Hemisferio Norte y en la Antártida, generando los clásicos episodios glaciales e interglaciales. Los datos paleontológicos y de isótopos de oxígeno sugieren que tanto la amplitud como la frecuencia en la variabilidad climática han alcanzado los máximos valores desde el pasado millón de años (Kennett, 1982; Fig. 1.6).

Durante la transición del Pleistoceno medio ("The mid-Pleistocene Transition", MPT), la cual comenzó hace 1250 Ka y terminó hace 600 Ka (Berger y Jansen, 1994), el clima de la Tierra sufrió profundos cambios en intensidad y duración de sus ciclos glaciales. El período de la MPT representa el período en el cual la señal climática en el registro geológico cambió la ciclicidad dominante de $41 \mathrm{Ka}$ (entre los 35 Ma y los $\sim 950 \mathrm{Ka}$ anteriores, Raymo et al., 1989; Zachos et al., 2001), a la ciclicidad dominada por la excentricidad (durante los últimos 950; Berger y Jansen, 1994, Imbrie et al., 1993). Este período ha sido indicado por el cambio positivo en el promedio de los valores de $\delta^{18} \mathrm{O}$ de foraminíferos bentónicos, generalmente adscrito a la expansión de los casquetes de hielo continentales; y a las más severas glaciaciones (Pisias y Moore Jr., 1981; Clark, 1999; Ashkenazy y Tziperman, 2004). Por lo tanto, esta transición se caracterizó por cambios globales en el sistema climático, incluyendo la disminución general de la temperatura en el océano superficial, en especial en las células de surgencia del Atlántico Norte y del océano tropical; un incremento en la aridez de África y Asia, en la intensificación monzónica (Clark et al., 2006) y del volumen de hielo global (Shackleton et al., 1990). Sin embargo, esta tendencia de enfriamiento fue interrumpida en los estadios 31 y 37 en donde la señal del $\delta^{18} 0$ fue inusualmente más liviana (Fig. 1.6). 


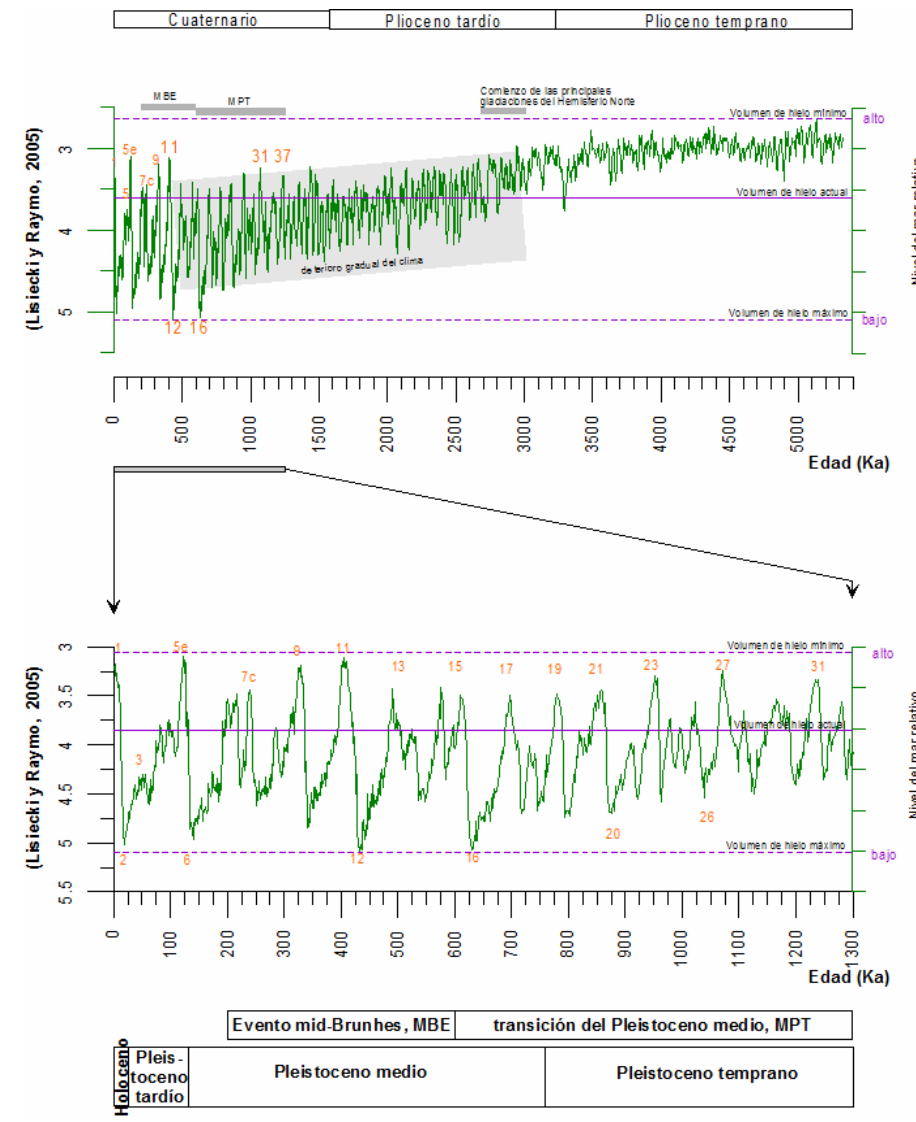

Fig 1.6. Arriba: Registro apilado del $\delta^{18} 0$ de foraminíferos bentónicos (Lisiecki y Raymo, 2005) usado como indicador del volumen del hielo, del clima y de los cambios del nivel del mar a lo largo de los últimos $5 \mathrm{Ma}(5000 \mathrm{Ka})$. Abajo: Ampliación del registro apilado $\delta^{18} \mathrm{O}$ a partir de los últimos $1300 \mathrm{Ka}$. Desde el comienzo de las principales glaciaciones en el Hemisferio Norte (3.0 a $2.7 \mathrm{Ma})$, la evolución del clima en la Tierra se caracteriza por a) el enfriamiento gradual de la Tierra desde hace unos $3 \mathrm{Ma}$ : b) Las fluctuaciones cíclicas del clima de la Tierra primero asociada al ciclo de oblicuidad y luego al de excentricidad. Ese cambio de frecuencia en la ciclicidad corresponde a la Transición del Pleistoceno medio (midPleistocene Transition, MPT); c) la transición del glacial 12 (que junto con el glacial 16 coinciden con los valores isotópicos máximos en el Cuaternario tardío) al interglacial 11 debido a su extrema amplitud. Evento midBrunhes (MBE, mid-Brunhes Event) asociado a la máxima acumulación y disolución del carbonato durante el Cuaternario tardío (Adoptado de Droxler et al., 2003).

El enfriamiento global atribuido a la MPT fue causado posiblemente por un descenso de la $P_{\mathrm{CO}_{2}}$ atmosférica y su influencia sobre el balance de radiación durante el Pleistoceno temprano (Raymo, 1997; Berger et al., 1999; Medina-Elizalde y Lea, 2005). Este enfriamiento ha sido muy evidente en las células de surgencia costera de los sectores de los océanos orientales ecuatoriales (Marlow et al., 2000; Liu y Herbert, 2004). Algunos autores han sugerido que el contraste de temperatura a través del océano Pacífico ecuatorial ha ejercido una influencia muy importante en la transición climática de la MPT (de Garidel-Thoron et al., 2005). Durante esta importante transición climática ocurrieron cambios significativos en la circulación termohalina (Hall et al., 2001), por ejemplo, descenso en la producción de la masa de Agua Profunda Noratlántica ("North Atlantic Deep Water", NADW) y un aumento en la producción de la masa de agua del océano del Sur. La formación de la NADW se debilitó durante las glaciaciones del Pleistoceno medio, favoreciendo el debilitamiento de la circulación y de la ventilación oceánica profunda (Schmieder et al., 2000), y el reforzamiento de la actividad biológica (Broecker, 1982; Knox y McElroy, 1984; Broecker y Peng, 1989; Sigman y Boyle, 2000). El período de la MPT también dio paso a la intensificación de la circulación atmosférica y aguas profundas en el océano del Sur (Raymo et al., 1990; Oppo y Lehman, 1993; Bertram et al., 1995; Hall et al., 
2001; Kleiven et al., 2003) y a la estratificación de las masas de agua en los océanos Atlántico (Oppo et al., 1995; Raymo et al., 1997; Flower et al., 2000), Pacífico e Índico (Keigwin, 1987; Duplessy et al, 1988; Kallen et al., 1988). Estas variabilidad en la circulación oceánica a escala glacial/interglacial modificó el perfil vertical de la distribución de nutrientes (Boyle, 1988), se reflejó en las asociaciones planctónicas y bentónicas, en particular, en la productividad primaria y por ende en la bomba biológica del carbono, en los niveles atmosféricos de $\mathrm{CO}_{2}$ y en las variaciones climáticas (Knox y McElroy, 1984; Broecker y Peng, 1989; Sigman y Boyle, 2000).

Entre los últimos $\sim 600$ y $\sim 200 \mathrm{Ka}$, en el océano global, se registró un período caracterizado por una severa disolución del carbonato a bajas y media latitudes (Fig. 1.6). Este período ha sido identificado como el Evento del Brunhes medio (“mid-Brunhes event”, MBE). El MBE también está marcado por un fuerte incremento en la acumulación de carbonato en el océano global. Algunos autores han indicado un incremento global en la producción del carbonato producido por el fitoplancton (cocolitóforos), en especial la proliferación del cocolitóforo Gephyrocapsa caribbeanica (Bollmann et al., 1998; Baumann y Freitag, 2004; Flores et al., 2003; Álvarez, 2005; entre otros) durante el MBE.

El MBE está centrado en el estadio isotópico marino ("Marine Isotopic Stage, MIS) 11 (Droxler et al., 2003). Diversos autores han sugerido otras condiciones climáticas inusuales durante el evento MBE. Tal es el caso de los estadios 11 y 9 que han sido considerados como estadios inusualmente cálidos dentro del último millón de años. Con relación a los estadios cálidos 5 y 1 , los estadios 11 y 9 han mostrado un intenso y prolongado calor, muy altos niveles del mar, una penetración inusual de aguas superficiales cálidas hacia los polos, y una rara y altísima acumulación de carbonato en los océanos profundos del Sur como se mencionó anteriormente (Droxler et al., 2003). El estadio 11 es un evento interglacial único porque además de haber sido tan cálido, fue probablemente el más largo del último millón de años. Fue un interglacial que demostró condiciones climáticas cálidas durante al menos $30 \mathrm{Ka}$, una duración dos veces mayor que las de los más recientes interglaciales 5e, 7c y Holoceno. El estadio 11 ocurrió después de un enfriamiento gradual de la Tierra que duró $2.5 \mathrm{Ma}$, y se caracterizó por el regreso de condiciones climáticas que fueron observadas probablemente antes de los $2.2 \mathrm{Ma}$ (Droxler et al., 2003; Mix et al., 1995). Durante el estadio 11 el nivel del mar pudo estar entre $13 \mathrm{~m}$ (Bowen, 2003) y 20 m (Hearty et al., 1999; Poore y Dowsett, 2001; Ortlieb et al., 2003) más alto que el actual, lo que indicaría que los casquetes de hielo del Ártico redujeron su extensión a valores mínimos. Esta combinación de ascenso del nivel del mar y aumento de las temperaturas 
superficiales del océano pudo favorecer el establecimiento de modernas barreras de arrecifes y atolones durante MIS 11. Sin embargo, los valores isotópicos del MIS 11 son similares 0 ligeramente más enriquecidos que los del Holoceno (Droxler et al., 2003)

EI MIS 11, no obstante, forma parte de un rompecabezas que no ha podido ser explicado ni modelado a través de la Teoría de Milankovitch, porque corresponde a un intervalo caracterizado por un mínimo de la excentricidad de la órbita de la Tierra y por el descenso general muy fuerte de la insolación entre $60^{\circ} \mathrm{N}$ y $70^{\circ} \mathrm{N}$. Lo cual hace pensar que la influencia externa fue demasiado débil como para reproducir la Terminación de más larga amplitud, la más desarrollada, de más duración y la más fuerte del intervalo interglacial del Cuaternario tardío (Droxler et al., 2003).

Mientras la MPT ocurrió gradualmente (Berger y Jansen, 1994), la transición del MIS 12 al MIS 11 está caracterizada por una transición única con la mayor amplitud de todos los registros glaciales (Droxler et al., 2003). El estadio glacial 16, también es un estadio inusual en el que los valores de $\delta^{180}$ fueron los más pesados que se registraron después del comienzo de la glaciación del Hemisferio Norte (3.0 a $2.7 \mathrm{Ma})$ y se repitieron durante el estadio glacial 12 (Droxler et al., 2003; Fig. 1.6).

El estadio interglacial 11 ha sido utilizado como un referente de los estadios interglaciales 5 y 1 en lo que se refiere al nivel del mar (como se explicó anteriormente), las temperaturas superficiales del océano y los niveles de $P_{\mathrm{CO}_{2}}$. McManus et al. (2003); Bauch y Erlenkeuser (2003); Hodell et al. (2003) indicaron que si el nivel del mar estuvo más alto durante el MIS 11, el océano tendría que haber sido más cálido; no obstante, sus registros sugirieron que las temperaturas del océano superficial y profundo fueron similares a las del Holoceno. Otros autores indicaron que las temperaturas del océano durante el MIS 11 fueron ligeramente más frías que durante los estadios 9, 5e y 1 (Droxler et al., 2003 y sus referencias). Basándose en los cambios de disolución del carbonato en las cuencas profundas del Atlántico Sur, Hodell et al. (2003) sugirieron niveles de $P_{\mathrm{CO}_{2}}$ atmosférico similares a las concentraciones preindustriales del Holoceno; sin embargo, resultados recientes del testigo de hielo antártico "Dome C", indican niveles máximos de $\mathrm{CO}_{2}$ de 275 ppmv dentro de los niveles normales de otros estadios descartando un efecto invernadero anormalmente alto para el MIS 11 (Epica community members, 2004). Otra diferencia entre el estadio 11 y el Holoceno es que el estadio 11 fue antecedido por un evento glacial extremo, el MIS 12, lo cual se explica a través de la relativa 
importancia del ciclo de oblicuidad en comparación a la baja amplitud del ciclo de precesión (Fig. 1.6).

Hodell et al. (2003) indicaron que hay que tener cuidado a la hora de establecer un análogo climático para el Holoceno y el clima futuro debido a que el sistema climático y el ciclo geoquímico fueron muy diferentes a aquellos del Pleistoceno tardío. McManus et al. (2003) por su parte, compararon los valores mínimos de insolación en el Hemisferio Norte durante el MIS 11 y los del interglacial actual, y propusieron que esos valores mínimos de insolación (la condición para que se desarrollaran los casquetes de hielo de acuerdo a la Teoría de Milankovitch) influyeron en que la criosfera se saltara un ritmo precesional durante el MIS 11, lo cual también podría repetirse dentro de $20 \mathrm{Ka}$.

\subsection{Eventos análogos al fenómeno de "E Niño" durante el Pleistoceno}

Las investigaciones relacionadas con el clima han dejado sobre la mesa un debate relacionado con la efectividad del trópico en la modulación de la variabilidad del clima global a diferentes escalas de tiempo. La variabilidad en el sistema de circulación oceánico-atmosférica en el Pacífico tropical tiene implicaciones globales por medio del transporte de calor y de vapor de agua, tal como ha sido demostrado a escala interanual por los eventos de "El Niño" (Cane, 1998; McClymont y Rosell-Melé, 2005). Adicionalmente, la variabilidad en el sistema de vientos, insolación, hidrografía y circulación, relacionada con los eventos ENSO en el Pacífico tropical oriental llega a ser de tal magnitud y duración que afecta a los organismos, poblaciones y ecosistemas (Wang y Fiedler, 2006). Los efectos biológicos de la ocurrencia del fenómeno ENSO han sido explicados a través del análisis de las comunidades fitoplanctónicas y las pesquerías comerciales. Chavez y Barber (1987) estudiaron los efectos biológicos producidos por "El Niño" durante 1982-1983, y Sánchez et al. (2000) aquellos ocurridos durante "El Niño" de 1997-1998 en el Pacífico oriental, dos períodos en los que "El Niño" fue el más intenso durante los últimos tiempos. Los autores observaron que las temperaturas superficiales del océano subieron hasta $9^{\circ} \mathrm{C}$, la termoclina, nutriclina y oxiclina se profundizaron y el nitrato en el océano superficial disminuyó, todo ello afectando la producción primaria y consecuentemente la supervivencia, reproducción y distribución de los organismos del resto de los niveles tróficos más altos. 
Otros estudios, han explicado los cambios en la oceanografía, los efectos biológicos y ecológicos y su relación con una variabilidad análoga al fenómeno ENSO ocurridos durante el Pleistoceno. Para ello, se han utillizado diversos indicadores micropaleontológicos o geoquímicos en los sedimentos de los testigos extraídos del océano, los cuales han demostrado la existencia de un fenómeno similar en el tiempo geológico.

Tudhope et al. (2001) utilizaron el registro fósil de corales Porites para investigar la existencia y la variabilidad de los eventos análogos a "El Niño" durante los últimos 130 Ka en Papua-Nueva Guinea. Los autores encontraron variaciones interanuales en el registro fósil, las cuales realmente reflejan caracterísiticas similares al fenómeno ENSO manifestadas por los corales actuales. Estas variaciones han reflejado cambios en la amplitud que pueden ser el resultado de efectos combinados de las variaciones orbitales de la precesión y del debilitamiento del fenómeno ENSO durante las condiciones frías glaciales

Beaufort et al. (2001) estudiaron los cambios registrados en la asociación cocolitoforal en varios testigos localizados a través de los océanos Pacífico e Índico ecuatoriales para los dos últimos ciclos climáticos. Su finalidad fue analizar la variabilidad en la dinámica de la termoclina basada en los cambios en la abundancia relativa del nanolito Florisphaera profunda - taxón que habita la zona fótica inferior -, los cambios en la paleoproductividad y su relación con los parámetros orbitales terrestres. Beaufort et al. (2001) encontraron una ciclicidad glacial/interglacial y precesional en sus registros micropaleontológicos. La ciclicidad precesional de la productividad estuvo asociada a la topografía este-oeste de la termoclina entre el Pacífico y el Índico. Las variaciones en la productividad fueron asociadas a eventos análogos al fenómeno ENSO persistente durante el MIS 5 en el océano Pacífico oriental.

Koutavas et al. (2002) y Stott et al. (2002) reconstruyeron registros de temperaturas superficiales del océano a partir de los registros de $\delta^{18} \mathrm{O}$ y Mg/Ca en el Pacífico ecuatorial, a escala milenial para los últimos $30 \mathrm{Ka}$ y $70 \mathrm{Ka}$ respectivamente. Los autores identificaron en sus registros los patrones caracterísiticos de eventos análogos a "El Niño" actual durante los eventos fríos (el último máximo glacial y los episodios estadiales respectivamente).

McClymont y Rosell-Melé (2005) de igual manera, reconstruyeron un registro de temperatura superficial del océano. Los autores utilizaron el índice de insaturación de las alquenonas para 
inferir las variaciones orbitales de la intensidad de la circulación en el Pacífico tropical y la variabilidad de los eventos similares al ENSO actual entre los $1.5 \mathrm{Ma}$ y $500 \mathrm{Ka}$. Los autores sugirieron un evento análogo a "La Niña" durante el Pleistoceno medio caracterizado por la intensificación de la circulación Walker.

Álvarez (2005) también estudió el registro de la asociación cocolitoforal en la zona de surgencia costera del Perú con el objetivo de analizar la evolución de la dinámica del océano superficial durante los últimos $800 \mathrm{Ka}$. Sus datos también apuntaron a un evento muy marcado durante el MIS 5, caracterizado por condiciones oligotróficas para la zona de surgencia del Perú, el cual relacionó con un evento análogo a "El Niño".

Pena et al. (2008) analizaron los registros geoquímicos de $\delta^{18} \mathrm{O}, \delta^{13} \mathrm{C}$ y $\mathrm{Mg} / \mathrm{Ca}$ de foraminíferos planctónicos en el oéano Pacífico oriental ecuatorial con el fin de estudiar las variaciones de la nutri-termoclina para los últimos $275 \mathrm{Ka}$. Los autores indicaron que durante las terminaciones, la termoclina estuvo asociada a aguas más salinas provenientes de la Antártida, indicando una relación con los eventos análogos a "La Niña" actual.

\section{Referencias}

Alley, R. 2006. Cambio climático brusco. Investigación y Ciencia. Temas 45: Cambio climático. 28-34.

Alvarez, M.C. 2005. Reconstrucción paleoceanográfica del margen continental del Pacífico durante los últimos 800 Ka mediante el análisis de asociaciones de cocolitóforos. Ph. D Thesis, Universidad de Salamanca, Salamanca, Spain, $151 \mathrm{pp}$.

Andruleit, H., Rogalla, U., Stäger, S. 2005. Living coccolithophores recorded during the onset of upwelling conditions off Oman in the western Arabian Sea. Journal of Nannoplankton Research 27, 1-14.

Ashkenazy, Y., Tziperman, E. 2004. Are the $41 \mathrm{kyr}$ glacial oscillations a linear response to Milankovitch forcing? Quaternary Science Reviews 23, 1879-1890.

Bauch, H.A., Erlenkeuser, H. 2003. Interpreting glacial-interglacial changes in ice volume and climate from Subartic Deep Water foraminiferal $\delta^{18} \mathrm{O}$. In: Droxler, A.W., Poore, R.Z., Burckle, L.H. (Eds.), Earth's climate and orbital eccentricity. The Marine Isotope Stage 11 question. American Geophysical Union. Washington D.C. pp. 69-85.

Baumann, K.-H., Freitag, T. 2004. Pleistocene fluctuations in the northern Benguela Current system as revealed by coccolith assemblages. Marine Micropaleontology 52, 195-215.

Beaufort, L., de Garidel-Thoron, T., Mix, A.C., Pisias, N.G. 2001. ENSO-like forcing on oceanic primary production during the Late Pleistocene. Science 293, 2440-2444.

Bé, A.W.H. 1977. An ecological, zoogeographic and taxonomic review of recent planktonic foraminifera. In: Ramsay, S. (Ed.), Oceanic Micropaleontology Vol 1. A. T. Academic Press. London. pp. 1100.

Berger, W.A., Jansen, E. 1994. Mid-Pleistocene climate shift: the Nansen connection. In: Johanessen, O.M., Muensch, R.D., Overland, J.E. (Eds.), The role of the polar oceans in shaping the global environment. Geophysical Monographic Series, American Geophysical Union, Wahington D.C. 85. pp. 295-311.

Berger, A., Li, X.S., Loutre, M.F. 1999. Modeling northern hemisphere ice volume over the last $3 \mathrm{Ma}$. Quaternary Science Reviews 18, 1-11. 
Bertram, C.J., Elderfield, H., Shackleton, N.J., MacDonald, J.A. 1995. Cadmium/calcium and carbon isotope reconstructions of the glacial northeast Atlantic Ocean. Paleoceanography 10, 563-578.

Bollmann, J., Baumann, K.-H., Thierstein, H. 1998. Global dominance of Gephyrocapsa coccoliths in the late Pleistocene: selective dissolution, evolution or global environmental change? Paleoceanography 13, 517-529.

Bond, G., Heinrich, H., Huon, S., Broecker, W., Labeyrie, L., Andrews, J., McManus, S., Clasen, S., Tedesco, K., Jantschik, R., Simet, C., Klas, M. 1992. Evidence for massive discharges of icebergs into the North Atlantic ocean during the last glaciation. Nature 360, 245-249.

Bond, G., Broecker, W., Johnsen, S., McManus, J., Labeyrie, L., Jouzel, J., Bonani, G. 1993. Correlations between climate records from North Atlantic sediments and Greenland ice. Nature 365, 143-147.

Bowen, D.Q. 2003. Uncertainty in Oxygen Isotope Stage 11 sea-level: an estimate of $\sim 13 \pm 2 \mathrm{~m}$ from Great Britain. In: Droxler, A.W., Poore, R.Z., Burckle, L.H. (Eds.), Earth's climate and orbital eccentricity. The Marine Isotope Stage 11 question. American Geophysical Union. Washington D.C. pp. 131-144.

Boyle, E.A. 1988. The role of vertical nutrient fractionation in controlling late Quaternary atmospheric carbon dioxide. Journal of Geophysical Research 93 (C12), 15701-15714.

Broecker, W.S. 1982. Glacial to interglacial changes in ocean chemistry. Progress in Oceanography 11 , 151-197.

Broecker, W.S. 1994. Massive iceberg discharges as triggers for global climate change. Nature 372, 421424.

Bröecker W. S., Van Donk J. 1970. Insolation changes, ice volumes, and the $0^{18}$ record in deep-sea cores. Reviews Geophysics and Space Physics 8, 169-198.

Bröecker, W.S., Denton, G.H. 1989. The role of ocean-atmosphere reorganizations in glacial cycles. Geochimica et Cosmochimica Acta 53, 2465-2501.

Bröecker, W.S., Peng, T.H. 1989. The cause of glacial to interglacial atmospheric CO2 change: a polar alkalinity hypothesis. Global Biogeochemical Cycles 3, 215-240.

Bröecker, W., Bond, G., Klas, M., Bonani, G., Wolfli, W. 1990. A salt oscillatior in the glacial Atlantic? 1, The concept. Paleoceanography 5, 469-477.

Burroughs, W.J. 2001. Climate Change: A multidisciplinary approach, Cambridge University Press, Cambridge, 314p.

Cane, M.A. 1998. A role for the tropical Pacific. Science 282, 59-61.

Chavez, F.P., Barber, R.T. 1987. An estimate of new production in the equatorial Pacific. Deep-Sea Research Part A, 34, 1229-1243.

Clark, P.U., Alley, R.B., Pollard, D. 1999. Northern Hemisphere icesheet influences on global climate change. Science 286, 1104-1111.

Clark, P., Arhcer, D., Pollard, D., Blum, J., Rial, J., Brovkin, V.,Mix, A., Pisias, N., Roy, M. 2006. The middle Pleistocene transition: characteristics, mechanisms, and implications for long-term changes in atmospheric $\mathrm{pCO}_{2}$. Earth Science Reviews 25, 3150-3184.

Craig H. 1953. The geochemistry of the stable carbon isotopes. Geochimica et Coschimica Acta 3, 53-92.

Criss R. 1999. Principles of Stable Isotope Distribution. Oxford University Press.

Dansgaard, W., White, J.W.C., Johnsen, S.J. 1989. The abrupt termination of the Younger Dryas climate event. Nature 339, 532-534.

Dansgaard W., Johnsen S. J., Clausen H. B., Dahl-Jensen D., Gundestrup N. S., Hammer C. U., Hvidberg C. S., Steffensen J. P., Sveinbjörnsdottir A. E., Jouzel J., Bond G. 1993. Evidence for general instability of past climate from a $250 \mathrm{kyr}$ ice-core record. Nature 364, 218-220.

de Garidel-Thoron, T., Rosenthal, Y., Bassinot, F., Beaufort, L. 2005. Stable sea surface temperatures in the western Pacific warm pool over the past 1.75 million years.

Droxler, A.W., Alley, R.B., Howard, W.R., Poore, R.Z., Burckle, L.H. 2003. Introduction : Unique and exceptionally long interglacial Marine Isotope Stage 11 : window into Earth warm future climate. In: Droxler, A.W., Poore, R.Z., Burckle, L.H. (Eds.), Earth's climate and orbital eccentricity. The Marine Isotope Stage 11 question. American Geophysical Union. Washington D.C. pp. 1-14.

Duplessy, C.N., Shackleton, N.J., Fairbanks, R.G., Labeyrie, L., Oppo, D., Kallen, N. 1988. Deep Water source variations during the last climatic cycle and their impact on the global deep water circulation. Paleoceanography 3, 343-360. 
Emiliani, C. 1954. Depth habitats of some species of pelagic foraminifera as indicated by oxygen isotope ratios. American Journal of Sciences 252, 149-158.

Emiliani, C., Shackleton, N.J. 1974. The Brunhes Epoch: Isotopic paleotemperatures and geochronology. Science 183, 511-514.

Epica Community Members. 2004. Eight glacial cycles form an Antarctic ice core. Nature 429, 623-628.

Ericson, D.B., Wollin, G. 1956. Correlation of six cores from the equatorial Atlantic and the Caribbean. Deep-Sea Research 3, 104-125.

Flores, J.A., Marino, M., Sierro, F.J., Hodell, D.A., Charles, C.D. 2003. Calcareous plankton dissolution pattern and coccolithophore assemblages during the last $600 \mathrm{kyr}$ at ODP Site 1089 (Cape Basin, South Atlantic): paleoceanographic implications. Palaeogeography, Palaeoclimatology, Palaeoecology 196, 409-426

Flower, B.P., Oppo, D.W., McManus, J.F., Venz, K.A., Hodell, D.A., Cullen, J.L. 2000. North Atlantic intermediate water circulation and chemical stratification during past 1 Myr. Paleoceanography 15, 388-403.

Funnell, B.M., Haslett, S.K., Kennington, K., Swallow, J.E., Kersley, C.L. 1996. Strangeness of the equatorial ocean during the Olduvai magnetosubchron (1.95 to $1.79 \mathrm{Ma}$ ). In: Moguilevsky, A., Whatley, R. (Eds.), Microfossils and oceanic environments. University of Wales, Aberystwyth, 93109

Hall, I.R., McCave, I.N., Shackleton, N.J., Weedon, G.P., Harris, S.E. 2001. Intensified deep Pacific inflow and ventilation in Pleistocene glacial times. Nature 412, 809-812.

Hays, J.D., Imbrie, J. Shackleton, N.J. 1976. Variations in the Earth's orbit: pacemaker of the Ice Ages. Science 194: 1121-1132.

Hearty, P.J., Kindler, P., Cheng, H., Edwards, R.L. 1999. A +20 m middle Pleistocene sea-level highstand (Bermuda and the Bahamas) due to partial collapse of Antarctic ice. Geology 27, 375-378.

Heinrich, H. 1988. Origin and consequences of cyclic ice rafting in the northeast Atlantic Ocean during the past 130,000 years. Quaternary Research 29, 143-152.

Hodell, D.A., Kanfoush, S.L., Venz, K.A., Charles, C.D., Sierro, F.J. 2003. The mid-Brunhes transition in ODP sites 1089 and 1090 (Subantarctic South Atlantic). In: Droxler, A.W., Poore, R.Z., Burckle, L.H. (Eds.), Earth's climate and orbital eccentricity. The Marine Isotope Stage 11 question. American Geophysical Union. Washington D.C. pp. 69-85.

Hoefs, J. 1997. Stable isotope geochemistry. Springer-Verlag, Berlin, 241p.

Hughen, K.A., Overpeck, J.T., Peterson, L., Trumbore, S. 1996. Rapid climate changes in the tropical Atlantic region during the last deglaciation. Nature 380, 51-54. doi: 10.1038/380051a0

Huybers, P., Wunsch, C. 2004. A depth-derived Pleistocene age model: Uncertainty estimates, sedimentation variability and non linear climate change. Paleoceanography 19, PA1028, doi: 10.1029/2002PA000857.

Imbrie, J., Hays, J. D., Martinson, D. G., McIntyre, A., Mix, A. C., Morley, J. J., Pisias, N. G., Prell, W. L., Shackleton, N.J. 1984. The orbital theory of Pleistocene climate: Support from a revised chronology of the marine $\delta^{18} \mathrm{O}$ record. In: Berger, A. (Ed.), Milankovitch and Climate, Part 1, Springer, New York, pp. 269-305.

Imbrie, J., Berger, A., Boyle, E.A., Howard, W.R., Kukla, G., Kutzbach, J., Martinson, D.G., Mclntyre, A., Mix, A.C., Molfino, B., Morley, J.J., Peterson, L.C., Pisias, N.G., Prell, W.L., Raymo, M.E., Shackleton, N.J. Toggweiler, J.R. 1993. On the structure and origin of major glaciation cycles 2. The 100,000-year cycle. Paleoceanography 8,699-735

Kallen, N., Labeyrie, L.D., Julliet-Laclerc, A., Duplessy, J.C. 1988. A deep hydrological front between intermediate and deep water masses in the glacial Indian Ocean. Nature 333, 651-655.

Keigwin, L.D. 1987. North Pacific Deep Water formation during the latest glaciation. Nature 330, 362-364.

Kennett, J.P. 1982. Marine Geology. Englewood Cliffs. Prentice-Hall. New Jersey, 813 p.

Kennett, J.P., Huddlestun, P. 1972. Late Pleistocene Paleoclimatology, foraminiferal biostratigraphy and tephrochronology, western Gulf of Mexico. Quaternary Res. 2, 38-69.

Kleiven, H.F., Jansen, E., Curry, W.B., Hodell, D.A., Venz, K. 2003. Atlantic thermohaline circulation changes on orbital to suborbital timescales during the mid-Pleistocene. Paleoceanography 18 , 1008. doi:10.1029/2001PA000629

Knox, F., McElroy, M. 1984. Changes in atmospheric CO2: influence of biota at high latitudes. Journal of Geophysical Research 89, 4629-4637. 
Koutavas, A. Lynch-Stieglitz, J., Marchitto Jr., T., Sachs, J.P. 2002. El Niño-like pattern in ice age tropical Pacific sea surface temperature. Science 297, 226-229.

Laskar, J. 1990. The chaotic motion of the Solar System: a numerical estimate of the size of the chaotic zones. Icarus 88: 266-291.

Lisiecki, L.E., Raymo, M.E. 2005. A Pliocene-Pleistocene stack of 57 globally distributed benthic $\delta^{18} \mathrm{O}$ records. Paleoceanography 20, doi:10.1029/2004PA001071.

Liu, Z., Herbert, T.D. 2004. High-latitude influence on the eastern equatorial Pacific climate in the early Pleistocene epoch. Nature 427, 720-723.

Lund, D.C., Mix, A.C. 1998. Millenial-scale deep water oscillations: Reflections of the North Atlantic in the deep Pacific from 10 to 60 ka. Paleoceanography 13, 10-19.

Lutgens, F.K., Tarbuck, E.J. 1998. The Atmosphere: an introduction to meteorology. Seventh edition. Prentice Hall Inc. New Jersey, 434p.

Mantua, N.J. Hare, S.R., Zhang, Y., Wallace, J.M., Francis, R.C. 1997. A Pacific interdecadal climate oscillation with impacts on salmon production. Bulletin of the American Meteorological Society 78 , 1069-1079

Marlow, J.R., Lange, C.B., Wefer, G., Rosell-Mele, A. 2000. Upwelling intensification as part of the Pliocene-Pleistocene climate transition. Science 290, 2288-2291.

Maslin, M.; Seidov, D.; Lowe, J. 2001. "Synthesis of the nature and causes of rapid climate transitions during the Quaternary". . Geophysical Monographic Series, American Geophysical Union, Washington D.C. 126: 9-52.

McAyeal, D.R. 1993. Binge-purge oscillations of the Laurentide ice sheet as a cause of the North Atlantic's Heinrich events. Paleoceanography 8, 775-784.

McClymont, E.L., Rosell-Melé, A. 2005. Links between the onset of modern Walker circulation and the mid-Pleistocene climate transition. Geology 33, 389-392.

McManus, J., Oppo, D. Cullen, J., Healey, S. 2003. Marine Isotope Stage 11 (MIS 11): analog for Holocene and future climate? In: Droxler, A.W., Poore, R.Z., Burckle, L.H. (Eds.), Earth's climate and orbital eccentricity. The Marine Isotope Stage 11 question. American Geophysical Union. Washington D.C. pp. 69-85.

Medina-Elizalde, M., Lea, D.W. 2005. The mid-Pleistocene Transition in the Tropical Pacific. Science 310, 1009-1012.

Milliman, J.D. Meade, R.H. 1983. Worldwide delivery of river sediment to the oceans. Journal of Geology 91, 1-21.

Mix, A.C., Pisias, N.G., Rugh, w., Wilson, J., Morey, A., Hagelberg, T.K. 1995. Benthic foraminifer stable isotope record from Site 849 (0-5 Ma): local and global climate changes. In L. Mayer, Pisias, N., Janacek, T. etal., Proc. ODP, Init. Repts., 138. Ocean Drilling Program. College Station TX, 371412.

North Greenland Ice Core Project Members. 2004. High resolution record of northern hemisphere climate extending into the last interglacial period. Nature 431, 147-151

Oppo, D.W., Lehman, S.J. 1993. Mid-depth circulation of the subpolar North Atlantic during the Last Glacial Maximum. Science 259, 1148-1152.

Oppo, D.W., Raymo, M.E., Lohmann, G.P., Mix, A.C., Wright, J.D., Prell, W.L. 1995. A $\delta^{13} \mathrm{C}$ record of Upper North Atlantic Deep Water during the past 2.6 million years. Paleoceanography 10, 373394

Ortlieb, L., Guzmán, N., Marquardt, C. 2003. A longer-lasting and warmer interglacial episode during Isotopic Stage 11: Marine terrace evidence in tropical western Americas. In: Droxler, A.W., Poore, R.Z., Burckle, L.H. (Eds.), Earth's climate and orbital eccentricity. The Marine Isotope Stage 11 question. American Geophysical Union. Washington D.C. pp. 157-180.

Paillard, D.L. 2001. Glacial cycles: towards a new paradigm. Reviews of Geophysics, 39: 325-346.

Paillard, D.L., Labeyrie, L., Yiou, P. 1996. Macintosh program perfoms time-series analyses. EOS Transactions, American Geophysical Union 77 (39), 379.

Pena, L.D., Cacho, I., Ferretti, P., Hall, M.A. 2008. ENSO-like variability during glacial terminations and interlatitudinal tele-connections. Paleoceanography, in press. doi: 10.1029.2008PA001620

Philander, G. 1995. El Niño and La Niña. In: Pirie, R.G. (Ed.), Oceanography: Contemporary Readings in Ocean Sciences, Oxford Univ. Press, Oxford, U.K., 72-87. 
Pisias, N.G., Moore Jr., T.C. 1981. The evolution of Pleistocene climate: A time series approach. Earth Planetary Science Letters 52, 450-458.

Pisias, N.G:, Mix, A.C., Zahn, R. 1990. Nonlinear response in the global climate system: Evidence from benthic oxygen isotopic record in core RC13-110. Paleoceanography 5, 147-160.

Poore, R.Z., Dowsett, H.J. 2001. Pleistocene reduction of polar ice caps: Evidence from Cariaco Basin marine sediments. Geology 29, 71-74.

Prell, W.L., Damuth, J.E. 1978. The climate-related diachronous disappearance of Pulleniatina obliquiloculata in Late Quaternary sediments of the Atlantic and Caribbean. Marine Micropaleontol. 3, 267-277.

Rasmusson, E.M. 1985. El Niño and variations in climate. American Scientist 73, 168-177.

Raymo, M.E. 1997. The timing of major climate terminations. Paleoceanography 12, 577-585.

Raymo, M.E., Ruddiman, W.F., Backman, J., Clement, B.M., Martinson, D.G. 1989. Late Pliocene variation in northern hemisphere ice sheet and north Atlantic deep water circulation. Paleoceanography 4, 413-446.

Raymo, M.E., Ruddiman,W.F., Rind, D. 1990. Climatic effects of reduced Arctic sea ice limits in the GISSII GCM. Paleoceanography 5, 367-382.

Raymo, M.E., Oppo, D.W., Curry, W. 1997. The mid-Pleistocene climate transition: a deep sea carbon isotopic perspective. Paleoceanography 12, 546-559.

Ropelewski, C.F., Halpert, M.S. 1987. Global and regional scale precipitation patterns associated with El Niño/Southern Oscilation. Monthly Weather Review 110, 1606-1626.

Rühlemann, C., Dickmann, B., Mulitza, S., Frank, M. 2001. Late Quaternary changes of western equatorial Atlantic surface circulation and Amazon lowland climate recorded in Ceara Rise deep-sea sedimentos. Paleoceanography 16, 293-305.

Sánchez, G., Calienes, R., zuta, S. 2000. The 1997-98 El Niño and its effects on the coastal marine ecosystem off Peru. California Cooperative Oceanic Fisheries Investigations Reports 41, 62-86.

Shackleton, N.J., Berger, A., Peltier, W.R. 1990. An alternative astronomical calibration of the Pleistocene timescale based on ODP Site 677. Transactions of the Royal Society of Edinburgh: Earth Sciences 81, 251-261.

Shackleton. N.J., Fairbanks, R.G., Chiu, T-C, Parrenin, F. 2004. Absolute calibration of the Greenland time scale: implications for Antarctic time scales and for $\Delta^{14} \mathrm{C}$. Quaternary Science Reviews 23, 1513-1522.

Schlitzer, R. 2000. Electronic Atlas of WOCE Hydrographic and Tracer Data now available. EOS Transactions, American Geophysical Union 81 (5), 45.

Schmieder, F., Dobeneck, T. von, Bleil, U. 2000. The Mid-Pleistocene climate transition as documented in the deep South Atlantic Ocean: initiation, interim state and terminal event. Earth and Planetary Science Letters 179, 539-549.

Sigman, D.M., Boyle, E.A. 2000. Glacial/interglacial variations in atmospheric carbon dioxide. Nature 407, 859-869.

Stott, L., Poulsen, C., Lund, S., Thunell, R. 2002. Super ENSO and global climate oscillation at millenial time scales. Science 297, 222-226.

Thurman, H., Burton, E.A. 2001. Introductory Oceanography, Nineth Edition, Prentice Hall, New Jersey, $554 p$.

Tudhope, A. W., Chilcott, C.P., McCulloch, M.T., Cook, E.R., Chappell, J., Ellam, R.M., Lea, D.W., Lough, J.M., Shimmield, G.B. 2001. Variability in the El Niño-Southern Oscillation through a glacialinterglacial cycle. Science 291, 1511-1517.

Urey, H.C. 1947. The thermodynamic properties of isotopic substances. Journal of Chemical Society 1947. 562-581.

Uriarte-Cantolla, A. 2003. Historia del clima de la Tierra. Servicio Central de Publicaciones del Gobierno Vasco. País Vasco, 306 pp.

Van Donk, J. 1977. ${ }^{18} \mathrm{O}$ as a tool for micropaleontologists. In: Ramsay, S. (Ed.), Oceanic Micropaleontology Vol 2. A. T. Academic Press. London pp. 1345-1370.

Wang, C., Fiedler, P.C. 2006. ENSO variability and the eastern tropical Pacific: A review. Progress in Oceanography $69,239-266$.

Warren, B. A. 1981. Deep Circulation of the World Ocean, in Evolution of Physical Oceanography, edited by Warren, B.A., Wunsch, C., MIT Press, Cambridge, pp. 6-40. 
Weaver, A.J. 1999. Millenial timescale variability in ocean/climate models. In: Clark, P.U., Webb, R.S., Keigwin, L.D. (Eds.), Mechanisms of global climate change at millenial time scales. American Geophysical Union, Washington D.C. pp. 285-300.

Wefer, G., Berger, W.H., Bijma, J., Fischer, G. 1999. Clues to ocean history: a brief overview of proxies. En: Fischer, G., Wefer, G., Use of proxies in paleoceanography - Examples from the South Atlantic. Springer-Verlag, Berlin, 1-68.

Wyrtki, K. 1975. El Niño - the dynamic response of the equatorial Pacific Ocean to atmospheric forcing. Journal of Physical Oceanography 5, 57-244.

Zachos, J.C., Pagani, M., Sloan, L., Thomas, E., Billups, K. 2001. Trends, rhythms and aberrations in global climate $65 \mathrm{Ma}$ to present. Science 292, 686-693. 


\section{Cocolitóforos}

\subsection{Introducción}

2.2. Clasificación de los cocolitóforos

2.3. Biología y bioquímica: Organización interna y externa de la célula

2.4. Producción y función de los cocolitos

2.5. Ciclo vital

2.6. Biogeografía y paleobiogeografía

2.7. Sedimentación de los cocolitos

2.8. Contribución de los cocolitóforos a los ciclos biogeoquímicos: la sedimentación del carbonato biogénico y la producción de $\mathrm{CO}_{2}$

2.9. Los cocolitóforos como indicadores paleoceanográficos y paleoambientales

Referencias 


\subsection{Introducción}

Los cocolitóforos son algas unicelulares, planctónicas, susceptibles de producir placas carbonatadas, clasificados dentro de la división Haptophyta, clase Prymnesiophyceae (Hibberd, 1976). La calcificación en las formas susceptibles de calcificar tiene lugar en las aguas eufóticas, durante la vida del organismo; tras su muerte, las placas se incorporan al flujo de partículas que descienden hacia el fondo del océano. Los restos de cocolitóforos son muy abundantes en los sedimentos del fondo oceánico, contribuyendo a revelar la composición de las comunidades que existieron en la zona eufótica. Desde su primer registro en el Triásico constituyen uno de los grupos marinos fitoplanctónicos, más importantes porque desempeñan un papel relevante como productores primarios en el océano, y en los ciclos biogeoquímicos marinos del carbono, funcionando como bombas biológicas de transporte del carbono orgánico y del carbonato hacia el sedimento (Baumann et al., 2005).

Los cocolitóforos pueden formar eclosiones masivas en algunos sectores, perjudicando a otros elementos de las biotas naturales y a las actividades pesqueras comerciales (Sáez et al., 2004). La distribución de los cocolitóforos ha cambiado mucho a través del tiempo geológico. Durante el Cretácico fueron muy cosmopolitas y diversos, y ocuparon tanto las aguas ecuatoriales como las polares, pero la mayoría de especies se extinguieron al final del Cretácico. Actualmente los cocolitóforos han recobrado su importancia en aguas oceánicas tropicales y templadas; sin embargo, no son tan diversos como en el Mesozoico, quizás debido a su competencia con otros organismos como las diatomeas 0 , tal vez debido a los grandes cambios paleogeográficos.

Los términos generales más comúnmente utilizados para describir las algas haptofitas fueron revisados en la guía de la terminología de los nanofósiles calcáreos de Young et al. (1997), de la cual se siguió aquí una transcripción literal:

$\checkmark$ Nanoplancton: hace referencia al plancton de talla comprendida entre 2 a $63 \mu \mathrm{m}$ de diámetro, dentro del cual se incluye informalmente a los cocolitóforos, dinoflagelados y crisofitas pero se excluye el picoplancton bacterial.

$\checkmark$ Nanoplancton calcáreo: nanoplancton que produce placas calcíticas.

$\checkmark$ Nanofósil hace referencia a los fósiles de diámetro menor a las $63 \mu \mathrm{m}$, excluyendo los fragmentos y los estadios juveniles de fósiles de mayor talla. 
$\checkmark$ Nanofósil calcáreo: resto fósil del nanoplancton calcáreo.

$\checkmark$ Cocolitóforo sustantivo que hace referencia al organismo del nanoplancton calcáreo cuya talla está comprendida entre los $3 \mu \mathrm{m}$ y $40 \mu \mathrm{m}$ (Brand, 1994), que pertenece a la división Haptophyta y que poseen placas calcificadas llamadas cocolitos en algún estadio de su ciclo vital; e.g. Umbilicosphaera hulburtiana.

$\checkmark$ Cocolitofórido: adjetivo referido a algo perteneciente a los cocolitóforos, e.g. la pigmentación cocolitofórida.

$\checkmark$ Cocolito: término establecido por Huxley en 1857 tras su observación al microscopio óptico, y que literalmente significa "piedra esférica". Cocolito es un término colectivo que incluye todas las placas calcíticas biomineralizadas producidas por las haptophytas actuales y extintas (de Vargas et al., 2007). Los cocolitos son morfológicamente muy diferentes, su rango de tallas comprende desde $2 \mu \mathrm{m}$ hasta $25 \mu \mathrm{m}$, y su composición calcítica es generalmente homogénea.

$\checkmark$ Haptofita: hace referencia al alga unicelular de la división Haptophyta; incluye todos los cocolitóforos y también formas no-calcificadas que se relacionan con esta división, e.g. Prymnesium, Phaeocystis, Pavlova, Chrysochromulina. La taxonomía de las Haptophytas se puede plantear de diversas maneras, y una de ellas es separándolas en tres grupos artificiales: Haptophytas actuales no mineralizadas, cocolitóforos actuales y cocolitóforos fósiles. La identificación de las haptofitas que no mineralizan se realiza atendiendo fundamentalmente a la organización del flagelo y del haptonema, y a la forma de la célula. En los cocolitóforos actuales la identificación se establece principalmente con base en la morfología del cocolito, especialmente en la combinación de los tipos de cocolitos (cocolitos con morfología y tamaño similares o diferentes en la misma célula) y la organización de la célula (Jordan et al., 1995; Jordan y Chamberlain, 1997). La clasificación de las haptofitas fósiles se restringe exclusivamente a la morfología de los nanofósiles calcáreos (Tappan, 1980; Perch-Nielsen, 1985a, b; Aubry, 1989; Pienaar, 1994; Jordan y Chamberlain, 1997). En las últimas décadas los estudios realizados en cultivos de haptofitas han permitido conocer mejor sus ciclos de vida y la morfología orgánica desarrollada en cada fase del ciclo vital, y con ello precisar el alcance y la validez de ciertos criterios y conceptos taxonómicos (Aubry, 1989; Jordan y Chamberlain, 1997). 


\subsection{Clasificación de los cocolitóforos}

El esquema de clasificación de los cocolitóforos utilizado en este trabajo está basado en Cavalier-Smith (1998), Edvarsen et al. (2000), Young y Bown (1997a, b) y Cros y Fortuño (2002), para las categorías superiores; y en la guía de la taxonomía de los cocolitóforos actuales de Young et al. (2003) para las categorías inferiores:

Reino Cromista Cavalier-Smith 1981 emend. Cavalier-Smith 1998 Subreino Cromobiota Cavalier-Smith 1991 Infrarreino Haptophyta Cavalier-Smith 1995 División Haptophyta Hibberd 1972 ex Edvardsen y Eikrem 2000 Clase Prymnesiophyceae Hibberd 1976 Hibberd 1976 emend. Cavalier-Smith 1996 Subclase Prymnesiophycidae Cavalier-Smith 1986 emend. Cavalier-Smith 1996 en CavalierSmith et al., 1996

Orden Prinsiales Young y Bown 1997

Familia Noelaerhabdaceae Jerkovic 1970

Emiliania Hay y Mohler, en Hay et al., 1967

Gephyrocapsa Kamptner, 1943

Pseudoemiliania (Kamptner, 1963) Gartner, 1969

Reticulofenestra Hay, Mohler y Wade, 1966

Orden Coccosphaerales Haeckel 1984. emend. Young y Bown 1997

Familia Coccolithaceae Poche 1913

Coccolithus Schwarz 1894

Familia Calcidiscaceae

Calcidiscus Kamptner 1950

Umbilicosphaera Lohmann 1902

Oolithotus Reinhardt, en Cohen y Reinhardt 1968

Familia Pleurochrysidaceae Fresnel y Billard 1991

Pleurochrysis Pringsheim 1955

Familia Hymenomonadaceae Senn 1900

Hymenomonas Stein 1878

Ochrosphaera Schussnig 1930

Jomonlithus Inouye y Chihara 1983

Orden Zygodiscales Young y Bown 1997

Familia Helicosphaeraceae Black 1971, emend. Jafar y Martini 1975

Helicosphaera Kamptner 1954

Familia Pontosphaeraceae Lemmermann 1908

Pontosphaera Lohmann 1902

Scyphosphaera Lohmann, 1902

Orden Syracosphaerales Hay 1977

Familia Syracosphaeraceae Lemmermann 1908

Syracosphaera Lohmann 1902 
Familia Calciosoleniaceae Kamptner 1937

Calciosolenia Gran 1912

Familia Rhabdosphaeraceae Ostenfeld 1899

Rhabdosphaera Haeckel 1894

Discosphaera Haeckel 1894

Acanthoica Lohmann 1903, emnd. Schiller 1913 y Kleijne 1992

Heterococolitos "incertae sedis"

Familia Alisphaeraceae Young, Kleijne y Cros, en Young et al., 2003

Alisphaera Heimdal 1973

Familia Umbellosphaeraceae Young y Kleijne, en Young et al., 2003

Umbellosphaera Paasche, en Markali y Paasche 1955

Familia Papposphaeraceae Jordan y Young 1990

Papposphaera Tangen 1972

Nanolitos

Familia Braarudosphaeraceae Deflandre 1947

Braarudosphaera Deflandre 1947

Familia Ceratolithaceae Norris 1965

Ceratolithus Kamptner 1950

Neosphaera Lecal-Schauder 1950

Nanolitos "incertae sedis"

Florisphaera Okada y Honjo 1973

\subsection{Biología y bioquímica: Organización intema y extema de la célula}

Como es normal en los organismos fitoplanctónicos, la organización celular consta de dos partes, el protoplasma y la cubierta celular. Dentro del protoplasma se encuentran el núcleo, los cloroplastos, las mitocondrias, el aparato Golgi y otros orgánulos celulares que están conectados al retículo endoplasmático y a los microtúbulos exoesqueléticos en una matriz fluida. La cubierta celular separa este sistema del ambiente protegiéndolo y facilitando el intercambio de material entre la célula y el ambiente (Pienaar, 1994; Young, 1994). Esta cubierta celular es el periplasto, y en los cocolitóforos suele estar rodeada por dos componentes que desempeñan la función de cubrir la célula: las placas orgánicas resistentes, situadas inmediatamente fuera del plasma, y las placas inorgánicas calcáreas que corresponden a los cocolitos (Young, 1994). La mayor parte de las especies de los cocolitóforos presentan placas orgánicas y cocolitos, pero se pueden encontrar células que carezcan de ellos (Pienaar, 1994); es el caso de las emilianias que presentan una fase sin cocolitos (Cros y Fortuño, 2002; Young y Henriksen, 2003). Los 
cocolitóforos se diferencian de las demás haptofitas y de otros grupos planctónicos por presentar cocolitos, al menos en alguna fase de su ciclo de vida (Fig. 2.1).

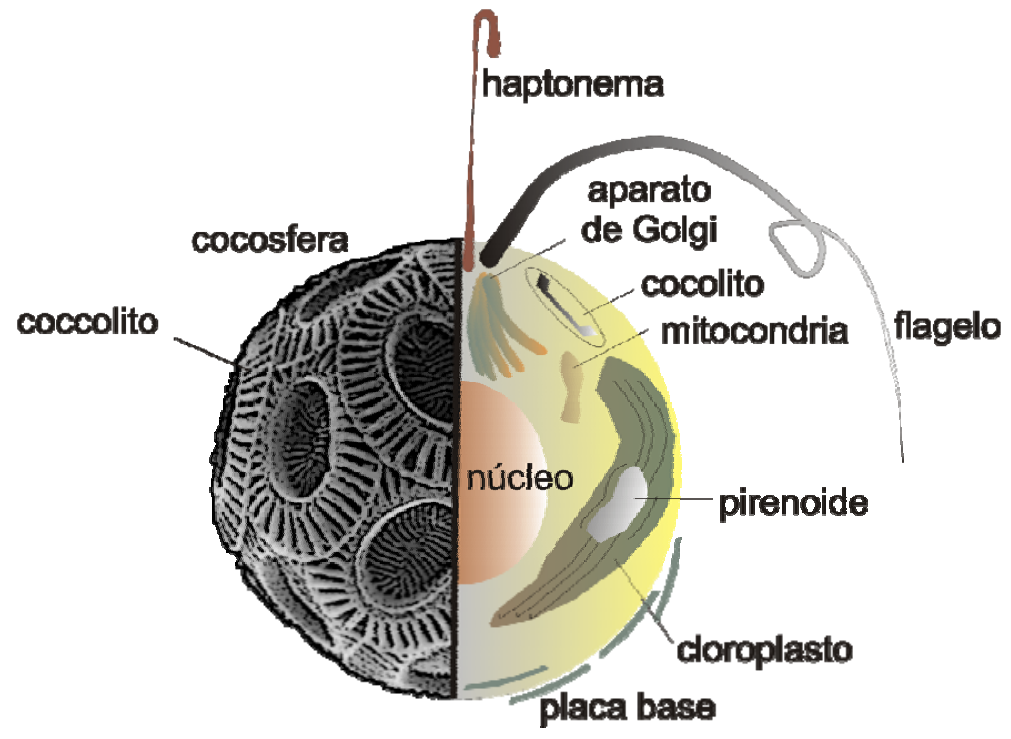

Fig. 2.1. Representación de la estructura de la célula de los cocolitóforos. Las características morfológicas del cocolito, las características citológicas y la definición de los organelos varían de acuerdo a cada taxón. Modificada de Flores y Sierro (2007).

El aparato flagelar está compuesto por dos flagelos casi siempre de igual o casi igual longitud en la clase Prymnesiophyceae, y un apéndice filiforme llamado haptonema situado entre los dos flagelos (Sáez et al., 2004). Los flagelos y el haptonema usualmente emergen de una depresión apical superficial (Pienaar, 1994). La presencia del haptonema es una de las características estructurales de las Prymnesiophyceae en aquellas células que tienen un estadio móvil. El haptonema es un orgánulo altamente variable, puede ser largo, corto, bulboso, liso o espiralado (Pienaar, 1994). Su función es poco conocida pero puede ser responsable de la fijación al sustrato; también puede ser un orgánulo táctil y sensorial, o posiblemente puede servir para la captura de materia particulada en algunas Prymnesiophyceae (Pienaar, 1994) (Fig. 2.1).

Los cloroplastos de las haptofitas contienen clorofilas "a" y "c1" y "c2" entre los pigmentos fotosintéticos y carotenoides como diadinoxantina y fucoxantina formando parte de los accesorios que le dan su color amarillo-pardo característico (Brand, 1994; Pienaar, 1994). Los clorosplastos pueden ser uno o dos y pueden tener pirenoide. El cloroplasto está rodeado por cuatro membranas, dos de las cuales representan el retículo endoplasmático del cloroplasto. El núcleo se encuentra en el centro y la parte final antapical de la célula (Pienaar, 1994; Billard e Inouye, 2004; Sáez, 2004). 
El aparato de Golgi está situado inmediatamente debajo de la región basal flagelar. Es responsable de la síntesis de las placas orgánicas y de la cocolitogénesis (Pienaar, 1994; Billard e Inouye, 2004) (Fig. 2.1).

\subsection{Produc ción y función de los cocolitos}

Los cocolitóforos están caracterizados por una cubierta externa de cocolitos que rodea la célula, la cocosfera (Pienaar, 1994) (Fig. 2.1) que no siempre es esférica. La producción de cocolitos es una importante característica bioquímica de los cocolitóforos y posiblemente este proceso puede influir en la fotosíntesis (Young, 1994) y tal vez en su ciclo biológico.

Los cocolitos incluyen tres grupos morfológicos holococolitos, heterococolitos y nanolitos, los cuales se diferencian no sólo por su forma, sino también por su modo de formación y por los estadios de los ciclos de vida durante los cuales se producen estos grupos morfológicos (Young y Bown, 1997a; Cros y Fortuño, 2002; Young et al., 2003; Flores y Sierro, 2007) (Fig. 2.2).

Los holococolitos se caracterizan por delgados microcristales romboédricos y hexagonales, idénticos en tamaño $(<0.1 \mu \mathrm{m})$ y forma (Aubry 1989; Jordan y Chamberlain, 1997), con una amplia diversidad morfológica y están organizados en ciclos que muestran una simetría radial. La placa base de los cristalolitos se produce en el aparato de Golgi, luego es expulsada al periplasto y la calcificación total se da extracelularmente. Los holococolitos son muy sensibles a la disolución durante su descenso a través de la columna de agua y posteriormente en la interfase agua/sedimento debido a su delicada morfología (Aubry, 1989; Cros y Fortuño, 2002).

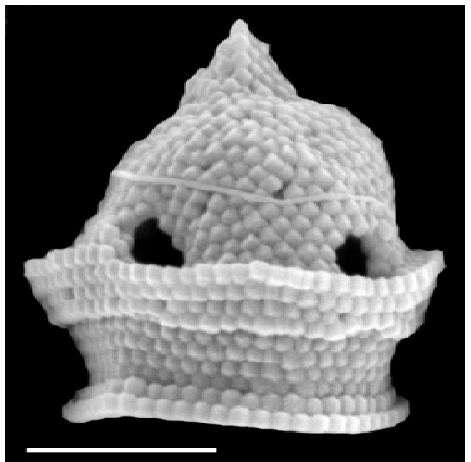

Holococolito

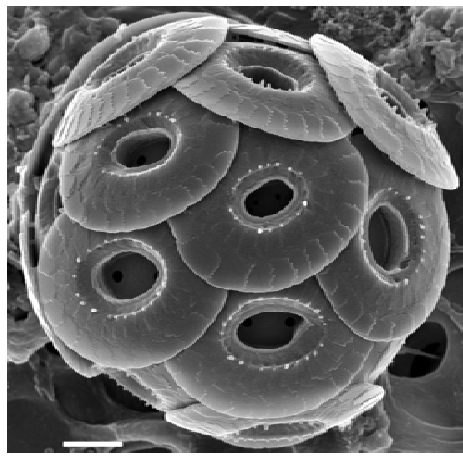

Heterococolito

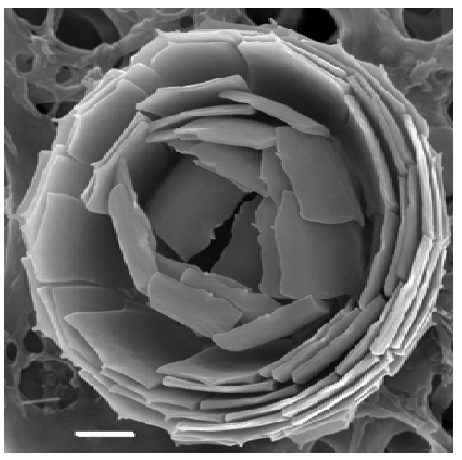

Nanolito

Fig. 2.2. Grupos morfológicos de los cocolitos. La escala blanca equivale a $1 \mu \mathrm{m}$. Las imágenes fueron adoptadas de http://www.nhm.ac.uk/hosted_sites/ina/galleries/3Dcoccos/index.htm y http://nannotax.org/ 
Los heterococolitos se caracterizan por delgados microcristales diferentes en tamaño y forma en el mismo taxón, lo cual parece indicar que la formación de los heterococolitos se controla genéticamente (Aubry, 1989); corresponden a las formas más comunes de los cocolitos encontradas en el sedimento, y se producen en el interior de la célula en el Aparato de Golgi o en el cuerpo reticular, migran a la región flagelar y luego son expulsados desde allí hacia el exterior de la célula, cuando están totalmente calcificados en las formas de cada especie (Geisen et al., 2002) (Fig. 2.1). Los heterococolitos morfológicamente constan de un área central y un borde. El área central puede poseer diferentes tipos de elementos como listones radiales, barras, etc., 0 incluso presentar estructuras altamente elaboradas o espinas. Los bordes difieren esencialmente en el ángulo que forman respecto al área central, así en los planolitos el borde externo se encuentra en el mismo plano y tiene dos o más escudos bien desarrollados; en los murolitos la mayor parte o todo el borde externo del disco es muy elevado y es perpendicular o casi perpendicular al área central y no tiene escudos desarrollados; y en los placolitos una pequeña parte del borde externo es perpendicular al área central, los escudos son paralelos al área central y están bien desarrollados (Cros y Fortuño, 2002) (Figs. 2.2, 2.3). Los nanolitos corresponden al tercer tipo morfológico de cocolitos. Están constituidos por cristales simples, diferentes a los de los heterococolitos y holococolitos que se han formado por diferentes procesos de biomineralización, y carecen de las estructuras típicas de los dinoflagelados, heterococolitos $u$ holococolitos y por lo tanto son de afinidad incierta (Cros y Fortuño, 2002; Flores y Sierro, 2007) (Fig. 2.2).
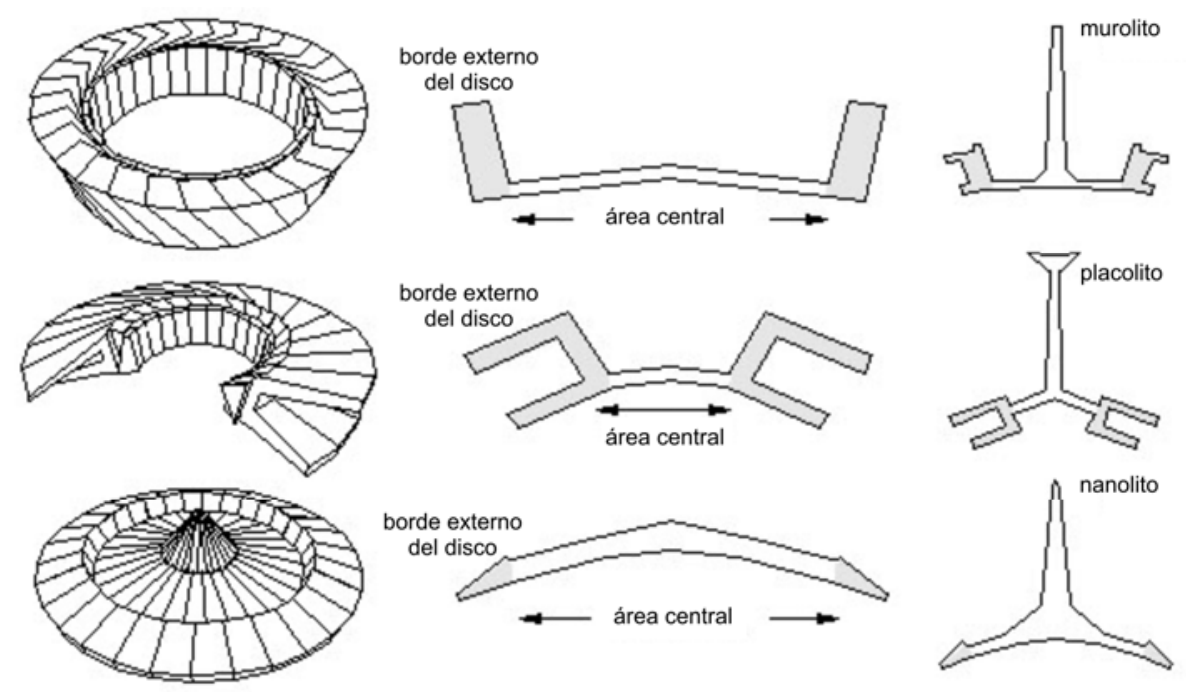

Fig. 2.3. Los principales morfotipos de los heterococolitos en sección vertical, los cuales no tienen implicaciones taxonómicas. Modificada de Young et al. (2003). 
La misma disposición de los cocolitos y la densidad de la cocosfera permiten la flotación dentro de la zona fótica para poder alimentarse. La arquitectura y disposición de los cocolitos han sido asociadas a la función protectora de la célula, reduciendo la influencia de parásitos, predadores, daños físicos, químicos, osmóticos, y de los rayos UV. Igualmente su organización podría estar asociada a la regulación de la luz: reflejándola para permitirles vivir en la zona fótica superior; 0 refractándola intracelularmente, permitiendo que algunas especies puedan vivir en la zona fótica inferior (Brand, 1994; Young, 1994). Durante las eclosiones masivas de cocolitóforos en algunas regiones del océano la reflexión de la luz puede alterar el albedo de la superficie del océano y aumentar temperatura de las aguas (Holligan et al., 1983). Aunque la producción de los cocolitos está fuertemente vinculada a la luz, no depende totalmente de ella; los procesos de calcificación requieren menos intensidad de luz que los procesos fotosintéticos. (Brand, 1994).

\subsection{Cic lo Vital}

Como es característico en las eucariotas, las células de los cocolitóforos presentan una alternancia de fases haploide y diploide en su ciclo de vida. Las células diploides tienen dos copias de cada cromosoma (2n); mientras que las células haploides tienen sólo una copia de cada cromosoma (n). La alternancia de una fase diploide a una fase haploide se da a través de la meiosis, en la cual se establece la división de la célula; mientras que el paso de una fase haploide a una diploide se establece a través de la singamia por la fusión de dos células (Young y Henriksen, 2003) (Fig. 2.4).

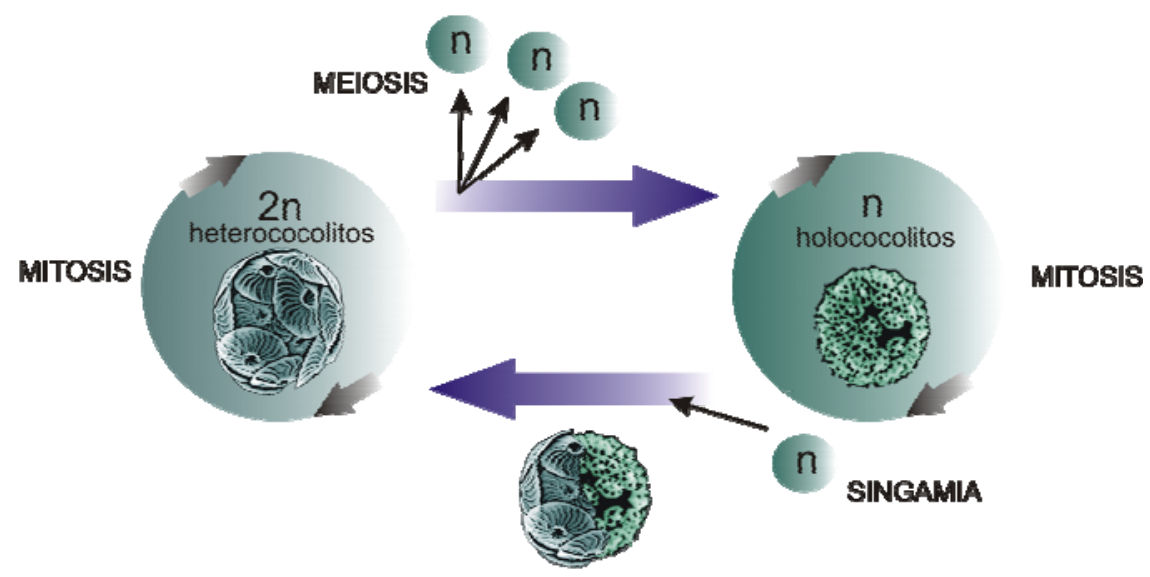

Fig. 2.4. Ciclo de vida de un cocolitóforo (Modificada de Flores y Sierro, 2007). El ciclo de vida de un cocolitóforo consiste de un estadio diploide que está cubierto de heterococolitos, y de un estadio móvil haploide que usualmente está cubierto de holococolitos. De esta manera se confirma que los holococolitóforos no son autónomos, como se pensaba, sino que representan estadios en el ciclo de vida de los heterococolitóforos. 
Muchos organismos presentan ciclos de vida en los cuales una de estas fases es la dominante; pero en las haptofitas, estas dos fases tienen el mismo peso y las dos pueden experimentar una división binaria asexual (mitosis) aparentemente de manera indefinida hasta que la influencia ambiental estimule la transición hacia la fase alterna del ciclo de vida. Por lo tanto, el ciclo vital de algunos cocolitóforos consiste en tener una fase haploide y una diploide (Young y Henriksen, 2003) (Fig. 2.4).

Las fases haploide y diploide reflejan diferentes modos de biomineralización en los cocolitos que cubren la célula. En algunos, la fase haploide está caracterizada por holococolitos y la fase diploide por heterococolitos (Calcidiscus, Coccolithus). Otros tienen una fase haploide con células "desnudas" y una fase diploide con heterococolitos (Pleurochrysis, Emiliania, Gephyrocapsa). $Y$ otros parecen tener ciclos de vida en los que intervienen fases con heterococolitos y fases con nanolitos (Alisphaera, Ceratolithus) (Cros y Fortuño, 2002; Young y Henriksen, 2003) (Fig. 2.4).

\subsection{Biogeografía y paleobiogeografía}

Los cocolitóforos constituyen un componente abundante del fitoplancton en aguas templadas y

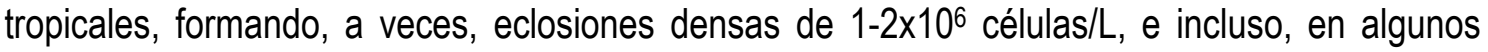
casos, de hasta $4 \times 10^{8}$ cocolitos/L, suficientemente densas para ser vistas por aviones y ser percibidas por satélites (Marchant y Thomsen, 1994).

Los cocolitóforos generalmente viven a lo largo de la zona fótica, la cual les permite realizar la fotosíntesis. La disposición temporal y espacial de estos organismos puede responder a las variaciones biológicas, las cuales están vinculadas a los factores ambientales primordiales en el océano, como son la temperatura y el régimen trófico (Brand, 1994, Young, 1994); aunque el amplio rango de tolerancia de temperatura y salinidad por parte de algunas especies (por ejemplo Emiliania huxleyi), parece indicar que la disponibilidad de nutrientes unidos a la temperatura y la intensidad de la luz son los factores esenciales en la distribución de los cocolitóforos en las masas de agua superficiales (Mclntyre et al., 1970).

En términos generales, algunos autores han diferenciado varias zonas biogeográficas de cocolitóforos distribuidas latitudinalmente en sus grandes rasgos (Fig. 2.5), aunque al estar 
separadas por las masas de agua en continuo movimiento, sus límites no corresponden exactamente a líneas rectas latitudinales (Winter et al., 1994; Ziveri et al., 2004). Mclntyre y Bé (1967) establecieron cinco asociaciones de cocolitóforos en el Atlántico de acuerdo con las asociaciones florísticas dominantes y la influencia de la zonación vertical de las especies: tropical, subtropical, transicional, subártica y subantártica. Okada y Honjo (1973) identificaron seis provincias biogeográficas en el Pacífico norte y tropical: central sur, ecuatorial sur, ecuatorial norte, central norte, transicional y subártica. Posteriormente, Winter et al. (1994) realizaron una integración y una descripción resumida de las provincias biogeográficas propuestas previamente para el Atlántico y el Pacífico; y Ziveri et al. (2004) presentaron una revisión de la distribución biogeográfica de cinco especies de cocolitóforos en el océano Atlántico. Finalmente, SaavedraPellitero (2006) identificó cinco zonas biogeográficas de cocolitóforos en el Pacífico suroriental y tropical: tropical norte, ecuatorial, tropical sur, subtropical sur, subpolar transicional. Las provincias biogeográficas descritas posteriormente a las de Mclntyre y Bé (1967), se caracterizan por presentar patrones similares a aquellas del Atlántico, por lo tanto, son reconocibles en los hemisferios norte y sur de los océanos mencionados, con excepción de las zonas polares (Subártica y Subantártica) (Winter et al., 1994; Fig. 2.5).

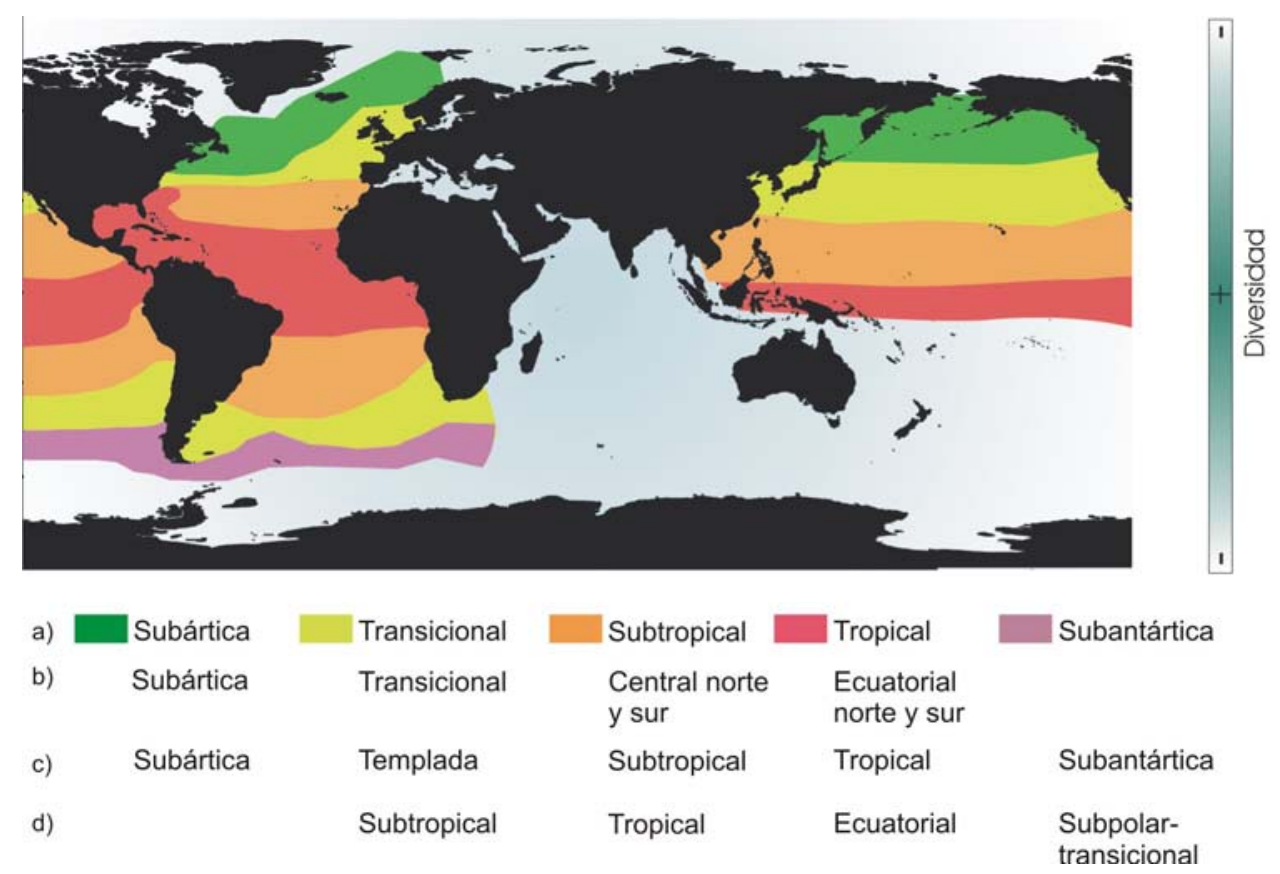

Fig. 2.5. Zonación biogeográfica de cocolitóforos actuales, de acuerdo a la terminología seguida por a) McIntyre y Bé, (1967), b) Okada y Honjo (1973), c) Winter et al. (1994) y d) Saavedra-Pellitero (2006). El mapa de fondo fue adoptado de Saavedra-Pellitero (2006).

De acuerdo con Winter et al. (1994), las provincias biogeográficas de cocolitóforos actuales se caracterizan de la siguiente manera: 
$\checkmark$ Zona Subártica: Caracterizada por la Familia Papposphaeraceae (en regiones costeras), Coccolithus pelagicus (que registra una abundancia máxima en la zona Subártica y está ausente en la Zona Subantártica) y Calciopapus caudatus (en el océano abierto).

$\checkmark$ Zona Templada: Caracterizada por placolitos, especialmente Emiliania huxleyi, Gephyrocapsa oceanica y Gephyrocapsa muellerae.

$\checkmark$ Zona Subtropical: Caracterizada por holococolitóforos, Discosphaera tubifera, Rhabdosphaera clavigera, Umbellosphaera spp., Florisphaera profunda, Umilicosphaera sibogae (máxima en el Atlántico Sur), Thorosphaera flabellata y Syracosphaera spp.

$\checkmark$ Zona Tropical: Caracterizada principalmente por placolitos, en especial, Emiliania huxleyi, Gephyrocapsa oceanica y Calcidiscus leptoporus. Otras especies como Florisphaera profunda, Helicosphaera carteri (con máxima abundancia en la zona Tropical) Umbellosphaera spp., y Thorosphaera flabellata también son importantes.

$\checkmark$ Zona Subantártica: Las únicas especies encontradas corresponden a las especies de la Familia Papposphaeraceae.

Los nutrientes en las zonas subpolares y transicionales están asociados a un intenso reciclaje de la materia orgánica en las aguas superficiales. Estos "nutrientes reciclados" ("regenerated production"; Eppley y Peterson, 1979) posibilitan una altísima producción del fitoplancton en el océano superficial, la cual reduce la penetración de luz a través de la capa de mezcla e impide la zonación vertical. Por el contrario, la zonas subtropicales están asociadas a la inyección de "nuevos nutrientes" ("new production") a la zona eufótica a través de las células de surgencia, el polvo atmosférico o las descargas fluviales (Eppley y Peterson, 1979). La llegada de "nuevos nutrientes" a las zonas subtropicales hacen que la productividad sea menor que en las altas latitudes pero permiten la distribución vertical de las asociaciones de especies en la zona fótica superior $(0-80 \mathrm{~m})$ y en la zona fótica inferior (120-220 m). La zona Tropical también está caracterizada por la influencia de la zonación vertical de las asociaciones, pero a profundidades más someras que las de las zonas subtropicales (Winter et al., 1994; Figs. 2.5, 2.6).

Brevemente se puede decir que los cocolitóforos se distribuyen de acuerdo a la disponibilidad de nutrientes desde aguas costeras y subpolares eutróficas hasta giros subtropicales oligotróficos, reflejando en sus características biológicas el tipo de estrategia adoptado (estrategia $r$ o estrategia k) (Brand, 1994); no obstante, la influencia de la variación en la temperatura superficial del océano en la distribución de la asociación cocolitoforal, no debe ser descartada. 


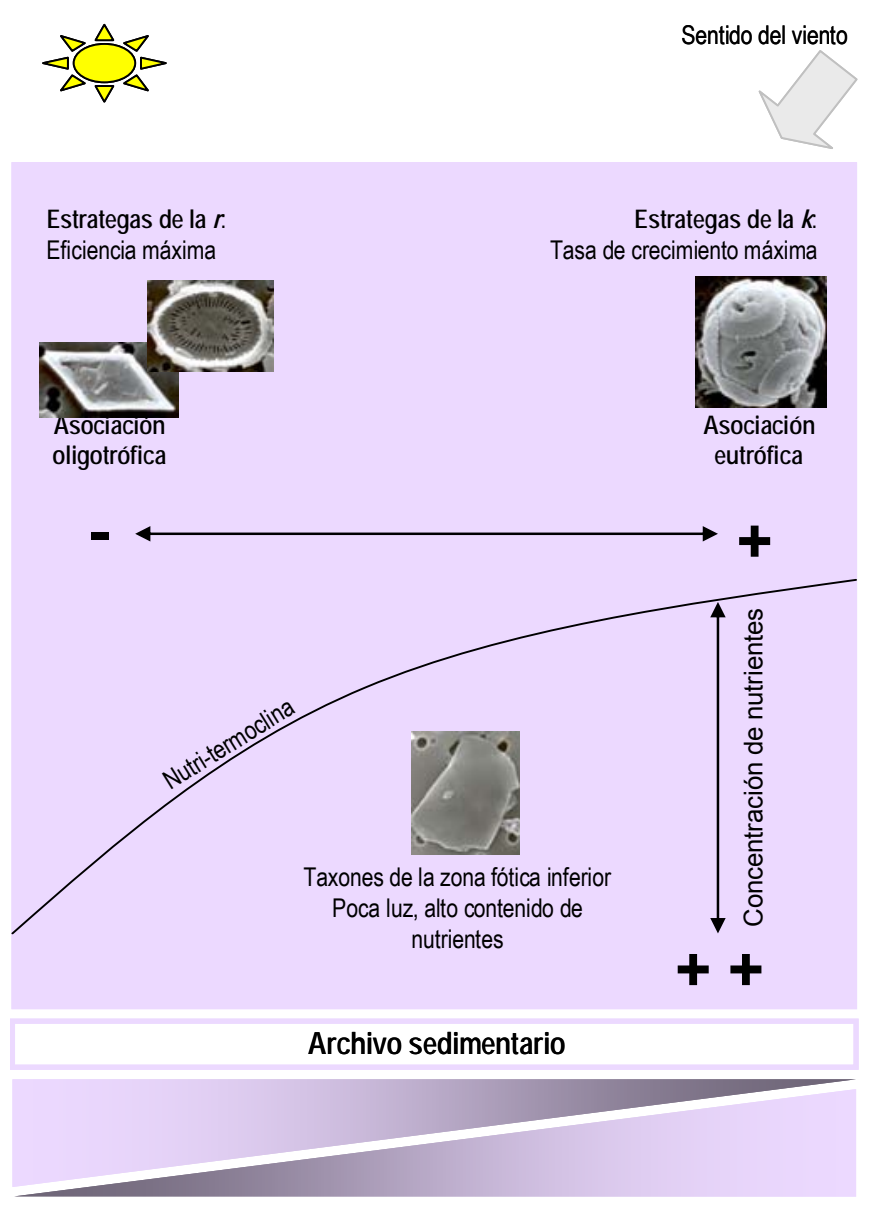

Fig. 2.6. Zonación vertical de los cocolitóforos en la columna de agua del océano superficial en las zonas subtropicales y tropicales (modificada de Kinkel et al., 2000). El sentido del viento favorece la formación de corrientes superficiales que arrastran masas de agua cálidas y las acumulan al final de su recorrido. El desplazamiento de las masas de agua cálidas permite la somerización de la nutritermoclina, el afloramiento de aguas enriquecidas en nutrientes y con ello el incremento de la productividad superficial. Mientras que la acumulación de esas masas de agua causa la profundización de la nutritermoclina, el descenso en la tasa de nutrientes disponibles en la zona fótica superior y por lo tanto una disminución en la productividad. Las imágenes de los taxones pertenecen a los testigos 1240 y 1241 y fueron tomadas en el Laboratorio de Paleontología de la Universidad de Kiel.

Tradicionalmente, los cocolitóforos han sido considerados como estrategas de la k, quizás porque su importancia relativa respecto a otros grupos se da al final de la sucesión ecológica, sin embargo, los placolitos son uno de los componentes mayoritarios de las asociaciones eutróficas (Young, 1994).

\subsection{Sedimentación de los c ocolitos}

La sedimentación de los cocolitos hacia el fondo oceánico depende de varios procesos físicos, químicos y biológicos, que pueden actuar a través de la columna de agua o durante el período posdeposicional. La disolución, resuspensión o los procesos ocurridos en la zona bentónica pueden afectar el flujo de cocolitos a través de la columna de agua; y su enterramiento, la compactación del sedimento, la bioturbación, los procesos físico-químicos en la interfase aguasedimento, las reacciones microbianas, la precipitación y la disolución, pueden actuar durante el período posdeposicional. La tasa de descenso aproximada en un cocolito de $3 \mu \mathrm{m}$, en un ambiente tranquilo donde los movimientos laterales de las masas de agua son mínimos, es alrededor de $14 \mathrm{~cm} /$ día (Flores y Sierro, 2007). Ésta baja velocidad sumada al hecho de que la 
relación superficie/masa de un cocolito es muy grande, provoca la aceleración del proceso de disolución de los cocolitos en aguas insaturadas respecto al carbonato (Honjo, 1975).

La presión hidrostática hace que la solubilidad de la calcita se incremente con la profundidad en la columna de agua; por ello, la disolución de la calcita se va tornando más intensa en profundidad (Farrell y Prell, 1989) haciendo que la diversidad de la asociación decrezca (Steinmetz, 1994). De este modo se pueden establecer dos horizontes en profundidad basados en la intensidad de disolución del carbonato (Figura 2.7). El primero que encontramos al descender es la llamada lisoclina, y coincide con la zona de transición entre las aguas superficiales saturadas en carbonato y otras más profundas del océano que están subsaturadas respecto al carbonato. Este horizonte de profundidad separa el los sedimentos donde el carbonato está relativamente bien preservado de aquellos donde éste se encuentra pobremente preservado (Berger, 1968). Al aumentar la profundidad bajo la lisoclina se alcanza otro horizonte llamado profundidad de compensación de la calcita (“Calcite Compensation Depth”, CCD) por debajo del cual la tasa de disolución del carbonato iguala la tasa de sedimentación o deposición del mismo, así pues, los sedimentos que se encuentran por debajo de esta profundidad no llegan a almacenar o acumular fósiles carbonatados (Brammlette, 1961).

La velocidad de sedimentación y por consiguiente la preservación de los cocolitos pueden aumentar cuando éstos descienden al fondo oceánico en forma de agregados fecales ("fecal pellets"), nieve marina o macroagregados. De esta forma permanecen menos tiempo en contacto con las condiciones agresivas para el $\mathrm{CaCO}_{3}$ del agua que se encuentra por debajo de la lisoclina. (Honjo, 1976; Steinmetz, 1994). Los agregados fecales son los excrementos del zooplancton, que entre otros restos de fitopláncton, contienen cocolitos relativamente bien conservados. Las partículas que conforman la nieve marina son partículas amorfas y frágiles con diámetros que varían desde $\sim 0.5 \mathrm{~mm}$ hasta algunos metros, y que incluyen partículas tan diversas como agregados macroscópicos, aglomerados, o floculados de materia orgánica 0 inorgánica (Knauer et al., 1984). Estas partículas se componen de bacterias, fitoplancton, zooplancton, agregados fecales, detritos, células algales libres, gránulos pigmentados, y nutrientes (Silver et al., 1978; Honjo et al., 1982). La sedimentación de las partículas al fondo oceánico es muy eficiente a través de los agregados fecales, pero en la práctica la nieve marina domina este proceso (Shanks y Trent, 1980; Honjo et al., 1984) como se ha puesto de manifiesto por ejemplo, en la cuenca de Panamá (Asper, 1987). 
Entre los cocolitos, los holococolitos son formas poco resistentes a la disolución, mientras que los heterococolitos y los nanolitos monocristalinos lo son más. En general, los cocolitos son más resistentes a la disolución que los foraminíferos planctónicos, siendo todos éstos más resistentes a la disolución que los pterópodos, lo cual se explica a través de la naturaleza del $\mathrm{CaCO}_{3}$ de los cocolitos. Además de tratarse de un carbonato bajo en magnesio, su estructura interna formada por un polímero parecido a la celulosa, y una disposición diferente de los ejes ópticos de los cristales de calcita, les confieren una mayor resistencia a la disolución comparada con otros carbonatos biogénicos (Steinmetz, 1994). Así, se observa una lisoclina más profunda para los cocolitos y los foraminíferos que para los pterópodos, cuyo carbonato cristaliza en forma de aragonito (Klocker et al., 2006).

\subsection{Contribución de los cocolitóforos a los ciclos biogeoquímic os: la sedimentación del carbonato biogénico y la producción de $\mathrm{CO}_{2}$}

Más del $90 \%$ del carbono total se acumula en los sedimentos marinos en forma de carbonato (Marchant y Thomsen, 1994). Los cocolitóforos, foraminíferos, pterópodos y dinoflagelados calcáreos son los organismos planctónicos más importantes en el aporte de carbonato en ambientes pelágicos, siendo los cocolitóforos y foraminíferos planctónicos los mayores aportadores (Milliman, 1993). El contenido del carbonato en sedimentos profundos está controlado por la relación que existe entre la producción, la dilución por material no carbonatado, y la disolución (Dittert et al., 1999; Heinrich et al., 2004).

El ciclo del carbonato en el océano está estrechamente vinculado al ciclo global del carbono, el cual controla los niveles de $\mathrm{CO}_{2}$ en la atmósfera, regula el pH y la constitución química del océano, principales condicionantes del desarrollo de los organismos calcáreos y de su metabolismo (Milliman, 1993).

El exceso de $\mathrm{CO}_{2}$ atmosférico puede ser neutralizado a través de varios procesos importantes en el océano. De forma pasiva, a través del intercambio de $\mathrm{CO}_{2}$ entre el océano superficial y la atmósfera (ecuación 1) y de manera activa, por la productividad primaria neta (fijación), por la respiración, por la producción (calcificación) y disolución de $\mathrm{CaCO}_{3}$, y por el intercambio de carbono entre las aguas superficiales y profundas a través de la circulación oceánica (Erez, 
2003). La presión del $\mathrm{CO}_{2}$ atmosférico está en equilibrio dinámico con la presión del $\mathrm{CO}_{2}$ en el agua, este proceso, a su vez, está regulado por el equilibrio ácido carbónico-bicarbonatocarbonato del agua superficial (Ecuación 1; Feely et al., 2001):

$$
\mathrm{CO}_{2}(g) \leftrightarrow \mathrm{CO}_{2}(a c)+\mathrm{H}_{2} \mathrm{O} \leftrightarrow \mathrm{H}_{2} \mathrm{CO}_{3} \leftrightarrow \mathrm{HCO}_{3}^{-}+\mathrm{H}^{+} \leftrightarrow \mathrm{CO}_{3}^{-2}+2 \mathrm{H}^{+}
$$

La suma total de todas estas especies es lo que se conoce como carbono inorgánico disuelto ("dissolved inorganic carbon", DIC; Ridgwell y Zeebe, 2005), y su equilibrio hace que los procesos que controlan el $\mathrm{CO}_{2}$ desde las aguas superficiales a las profundas, regulen también el $\mathrm{CO}_{2}$ desde la atmósfera hacia el océano (Feely et al., 2001; Fig. 2.7).

La fotosíntesis marina y la oxidación de materia orgánica están estrechamente vinculadas a la calcificación y la disolución, controlando el pH de los océanos y los niveles de $\mathrm{CO}_{2}$ atmosférico (Erez, 2003):

$$
\begin{array}{ll}
\mathrm{Ca}^{+2}+2 \mathrm{HCO}_{3}^{-} \leftrightarrow \mathrm{CaCO}_{3}+\mathrm{H}_{2} \mathrm{O}+\mathrm{CO}_{2} & \text { disolución } \leftrightarrow \text { calcificación (2) } \\
\mathrm{H}_{2} \mathrm{O}+\mathrm{CO}_{2} \leftrightarrow \mathrm{CH}_{2} \mathrm{O}+\mathrm{O}_{2} & \text { respiración } \leftrightarrow \text { fotosíntesis (3) }
\end{array}
$$

[donde: $\mathrm{CH}_{2} \mathrm{O}$ corresponde a una representación simplificada de la materia orgánica]

Los procesos de calcificación, fotosíntesis, respiración y parte de oxidación de la materia orgánica ocurren en el océano superficial. El proceso de calcificación en el océano actual es casi totalmente biogénico y su efecto sobre el pH marino puede liberar $\mathrm{CO}_{2}$ a la atmósfera (reacción 2; Erez, 2003; van Cappellen, 2003). Los productores primarios extraen el $\mathrm{CO}_{2}$ del océano superficial mediante la fotosíntesis marina (reacción 2) y lo excretan en los procesos metabólicos de la respiración (reacción 3; Ridgwell y Zeebe, 2005); pero la descomposición de la materia orgánica vuelve a disolverlo en el agua profunda, en donde suele ocurrir la disolución del $\mathrm{CaCO}_{3}$ (reacción 2; Brown et al., 1989; Erez, 2003). El hecho de que las aguas profundas estén sobresaturadas en $\mathrm{CO}_{2}$ en relación a la atmósfera también se debe a su largo aislamiento de la superficie y a la progresiva acumulación de $\mathrm{CO}_{2}$ respiratorio (Schlesinger, 2000). Por ello, la solubilidad del $\mathrm{CaCO}_{3}$ aumenta con la profundidad (como se mencionó en la sección 2.8): los sedimentos carbonatados son abundantes en las regiones más someras del fondo oceánico y está ausente en las llanuras abisales profundas. $\mathrm{El} \mathrm{CaCO}_{3}$ adicionalmente, es más soluble en las masas de agua frías que en las cálidas y es más soluble a mayor que a menor presión (los iones 
calcio y carbonato ocupan menos volumen cuando están disueltos que cuando están unidos en forma sólida; Brown et al., 1989).

El $\mathrm{CO}_{2}$ liberado puede ser reabsorbido después de la disolución del $\mathrm{CaCO}_{3}$ en profundidad y de la activación de las células de surgencia de aguas profundas. La escala de tiempo de este proceso es del orden de $1 \mathrm{Ka}$ (Broecker y Peng, 1982; Erez, 2003).

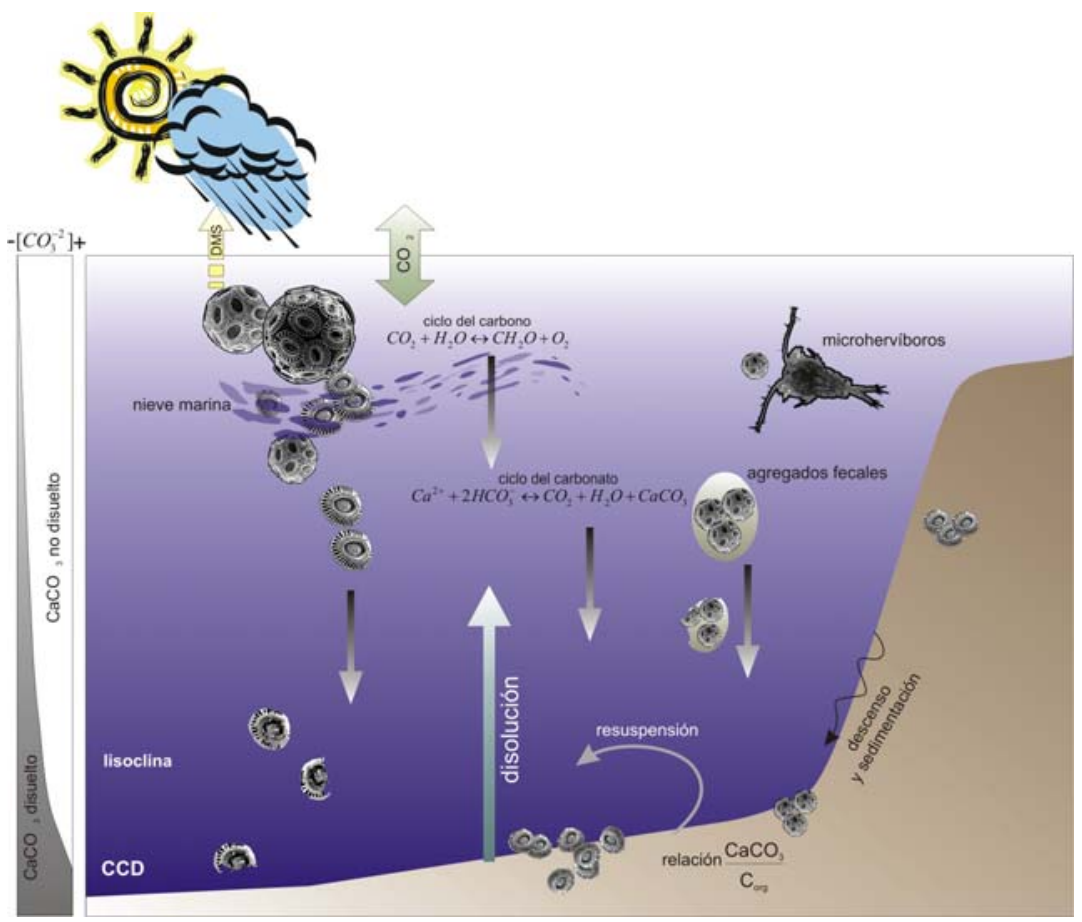

Fig. 2.7. Representación esquemática del papel de los cocolitóforos en las bombas biológicas del carbono y del carbonato. CCD: "Calcite Compensation Depth" (Profundidad de compensación de la calcita). Modificada de Flores y Sierro (2007).

El $\mathrm{CO}_{2}$ también se incrementa en profundidad debido a la disolución de $\mathrm{CO}_{2}$ atmosférico en las aguas frías de las altas latitudes, donde tienen su origen, una vez descienden al océano profundo tras incrementar su densidad (Brown et al., 1989).

La mayor parte del DIC en el agua marina está en forma de iones bicarbonato (Brown et al., 2005), cuando el DIC se incrementa, dentro de los niveles normales del océano, el pH no tiene por que variar al aumentar el DIC. Si se aumenta uno de los compuestos a un lado de la ecuación, por causas externas al sistema, por ejemplo, el $\mathrm{CO}_{2}$, entonces desciende el pH y la relación de los iones bicarbonato/carbonato se incrementa y también es más probable que el $\mathrm{CaCO}_{3}$ se disuelva. Un descenso de la $\left[\mathrm{CO}_{2}\right]$, por causas externas, es decir un aumento considerable de la fotosíntesis, supone el proceso contrario, el aumento del $\mathrm{pH}$. El equilibrio del $\mathrm{CO}_{2}$ atmosférico y el $\mathrm{CO}_{2}$ disuelto en el agua depende, entre otros factores, de la proporción 
relativa entre la fijación de $\mathrm{CO}_{2}$ por parte de la fotosíntesis, la precipitación biogénica de carbonato y la respiración.

La relación $\mathrm{CaCO}_{3} /$ carbono orgánico ("rain ratio") de la materia que se sedimenta desde el océano superficial, se usa como indicador de la $\left[\mathrm{CO}_{2}\right]$. Cuando este cociente decrece (sea debido a una menor calcificación o a una mayor producción de carbono orgánico), se asocia a una menor presión del $\mathrm{CO}_{2}$ en aguas superficiales, lo que indica una alcalinización del océano; el fenómeno opuesto supone una mayor concentración de $\mathrm{CO}_{2}$. Todo ello ha permitido que algunos autores sugieran que la precipitación del carbonato produce un incremento del $\mathrm{CO}_{2}$ en el océano, y con ello un incremento en la concentración de $\mathrm{CO}_{2}$ atmosférico; y de manera contraria, la disolución del $\mathrm{CaCO}_{3}$ favorece un descenso del $\mathrm{CO}_{2}$ (y por lo tanto, del $\mathrm{CO}_{2}$ atmosférico; Van Capellen, 2003; Ridgwell y Zeebe, 2005; Brown et al., 2005).

El nanoplancton calcáreo como productor primario y productor de carbonato cálcico influye en los ciclos biogeoquímicos y en el sistema climático global, desempeñando un papel importante en el ciclo del carbono: consume $\mathrm{CO}_{2}$ durante la fotosíntesis e influye en la alcalinidad del océano al producir carbonato cálcico. Además contribuye al transporte neto de carbono y alcalinidad hacia los sedimentos (Brand, 1994; Young, 1994; Flores y Sierro, 2007).

\subsection{Los cocolitóforos como indic adores paleoc eanográfic os y paleoambientales}

El registro geológico de los nanofósiles calcáreos desde el Triásico hasta la actualidad, ha mostrado gran abundancia y presencia de géneros o especies con distribución estratigráfica restringida entre los taxones más cosmopolitas, lo cual ha permitido que sean utilizados como herramientas bioestratigráficas muy útiles (Bown et al., 2004). El carbonato cálcico de los cocolitos preservados en el sedimento, también puede aportar información paleoecológica, paleoceanográfica y paleoclimática. Uno de los prerrequisitos para obtener esta información está relacionado con el conocimiento previo de la ecología de los cocolitóforos y los procesos que interfieren en la transformación de las asociaciones vivas (biocenosis) a las fósiles, las cuales forman parte de los sedimentos del fondo del océano (tanatocenosis). Algunos estudios han analizado la distribución espacial del nanoplancton observado en los sedimentos superficiales del océano (Mclntyre y Bé, 1967; Samtleben y Schröeder, 1992; Baumann et al., 2000; Sprengel 
et al., 2002; Stoll et al., 2007), mientras que otros (Ziveri et al., 2000, 2004; Sprengel et al., 2002; Stoll et al., 2007) han estudiado la variabilidad temporal (por ejemplo estacional o mensual) de la asociación de cocolitóforos actuales mediante el uso de trampas de sedimento localizadas a lo largo de la columna de agua. Este par de técnicas ha aportado información directa sobre la ecología de las formas actuales, permitiendo relacionar la dinámica oceanográfico-atmosférica que ha intervenido en la producción de los cocolitóforos hoy en día (Samtleben et al., 1995) con el registro geológico, lo que les confiere gran potencial para los estudios paleoecológicos y paleoceanográficos (Balestra et al., 2004). El Apéndice del capítulo 8 explica el significado paleoecológico de las diferentes especies observadas en los sedimentos del Pacífico oriental y Atlántico occidental tropicales estudiados en esta Tesis Doctoral.

Existen otras herramientas que han permitido precisar a alta resolución el significado paleoecológico del grupo, tales como las biometrías, el estudio de formas concretas dentro de la asociación, las estimaciones del carbonato aportado por este grupo de organismos, el análisis de las características geoquímicas a partir de las placas calcíticas y de biomarcadores producto de su actividad metabólica.

Las diferencias biométricas de la especie Coccolithus pelagicus s.l., estudiadas tanto en sedimentos superficiales com en testigos sedimentarios del Atlántico Norte, han sido utilizadas como un indicador paleoecológico y paleoceanográfico: el predominio en la abundancia del morfotipo pequeño (6-9 $\mu \mathrm{m}$; C. pelagicus pelagicus) indica la influencia de masas de agua atlánticas, el morfotipo intermedio (10-13 $\mu \mathrm{m}$; C. pelagicus braarudii) puede estar relacionado con cambios en la intensidad y/o posición del upwelling costero de lberia, y el morfotipo más grande (14-15 $\mu \mathrm{m}$, C. pelagicus azorinus) parece estar relacionado con la influencia del Frente de las Azores (Parente et al., 2004; Narciso et al., 2006). Igualmente, otros estudios biométricos se han dedicado a observar el comportamiento paleoecológico de otros taxones en sus diversos rangos morfométricos, tal como ha sido explicado para Calcidiscus leptoporus, Helicosphaera carteri (Knappertsbusch et al., 1997) y Emiliania huxleyi (Colmenero-Hidalgo et al., 2002).

El estudio de la abundancia relativa de Florisphaera profunda (Molfino y Mclntyre, 1990a, b; Okada y Matsuoka, 1996; Beaufort et al., 1997; Flores et al., 2000; Kameo et al., 2004; Martínez et al., 2005; entre otros), uno de los habitantes de la zona fótica inferior en los océanos tropicales y subtropicales (Okada y Honjo, 1973; Okada y McIntyre, 1977), y su relación con los habitantes de la zona fótica superior (los taxones de la Familia Noelaerhabdaceae) ha permitido realizar 
estimaciones de paleoproductividad. La disponibilidad de nutrientes en la zona fótica superior está controlada por la posición en profundidad de la nutri-termoclina y ésta a su vez por la activación de las células de surgencia, las cuales pueden ser trazadas a través de la relación de la abundancia de los Noelaerhabdaceae y F. profunda expresada a través del índice N (Flores et al., 2000, como se explica en los capítulos 5, 6 y 7). F. profunda ha sido utilizada adicionalmente como un indicador de la turbidez del agua del océano superficial en el Pacífico Noroccidental, en donde la transparencia de las masas de agua superficiales ha podido estar influida por la productividad biológica o por el aporte de partículas provenientes del continente (Ahagon et al., 1993).

La variabilidad en la abundancia relativa de ciertas especies del género Gephyrocapsa, ha sido utilizada con anterioridad en la reconstrucción cualitativa de las temperaturas de las masas de agua superficiales del océano (Weaver y Pujol, 1988; Takahashi y Okada, 2000), y ha revelado un patrón glacial/interglacial, indicando el descenso de las temperaturas durante los episodios glaciales. Bollmann et al. (2002) posteriormente, propusieron una calibración global de temperatura a través de una función de transferencia basada en la abundancia relativa de las especies del género Gephyrocapsa, la cual permitió inferir la migración de la célula de surgencia costera cercana al África Noroccidental durante el último máximo glacial y el Holoceno (Hendericks y Bollmann, 2004).

La técnica de las estimaciones del carbonato aportado por los cocolitos (Young y Ziveri, 2000) ha sido utilizada en los sedimentos del Pleistoceno medio y tardío en diferentes células de surgencia, donde el carbonato de los cocolitos es el componente sedimentario dominante. Los máximos flujos de carbonato derivado de los cocolitos están asociados a un aumento fuerte de la paleoproductividad y de la intensificación del régimen eutrófico en el Pacífico oriental (tal como se explica en los capítulos de discusión de resultados de esta Tesis), en Benguela (Baumann y Freitag, 2004) y en el océano global (Rickaby et al., 2007) durante el Evento del Brunhes medio ocurrido entre los estadios isotópicos marinos 15 a $8(\sim 600 \mathrm{Ka}$ a $200 \mathrm{Ka})$.

El carbonato aportado por los cocolitos también ha sido utilizado en estudios de geoquímica elemental de cocolitos, con la finalidad de reconstruir la química y las paleotemperaturas de las masas de agua en las que crecieron. La geoquímica de los cocolitos se diferencia de la de los foraminíferos principalmente en que la producción de calcita en los cocolitos es intracelular y como resultado tiene un fuerte control celular (Geisen et al., 2002; Billard e Inouye, 2004), lo cual 
indica que sobre la calcita de los cocolitos han intervenido procesos biológicos únicos durante el fraccionamiento isotópico. A pesar de ello, se ha demostrado que la técnica geoquímica puede ser utilizada como un buen indicador de la paleoproductividad de los cocolitóforos $(\mathrm{Sr} / \mathrm{Ca})$, de las paleotemperaturas de crecimiento de estos organismos $(\mathrm{Mg} / \mathrm{Ca})$, de la concentración de $\mathrm{CO}_{2}$ en el agua y en la atmósfera (isótopos de oxígeno) y en el estudio del ciclo del carbono entre el océano y otros reservorios (isótopos de carbono) (Stoll y Ziveri, 2004).

Algunos cocolitóforos también pueden aportar información paleoambiental a partir de sus restos orgánicos (fósiles moleculares o biomarcadores) incorporados en el sedimento. Emiliania huxleyi (Volkman et al., 1980a, b; Marlowe et al., 1984) y Gephyrocapsa oceanica (Conte et al., 1994; Volkman et al., 1995) son los principales organismos existentes biosinterizadores de un grupo de biomoléculas (de naturaleza lipídica) llamadas alquenonas. Se trata de etil- y metil-cetonas de 37 a 39 átomos de carbono con un número de insaturaciones con configuración trans que va de dos hasta cuatro (Boon et al., 1978; Marlowe et al., 1990). Se observó que en cultivos de ciertas especies de haptofitas, el grado de insaturación (contenido en dobles enlaces) de estas cetonas disminuía con la temperatura. Así de definieron dos índices, llamados $U_{37}^{k}$ y $U_{37}^{k^{\prime}}$ basados únicamente en las metil cetonas 0 alquenonas homólogas de 37 átomos de carbono (Prahl y Wakeham, 1987). El índice de insaturación de las alquenonas preservadas en los sedimentos marinos permite estimar la paleotemperatura del agua del océano en la que crecieron las haptofitas productoras y su paleoproductividad. El índice de insaturación no está influido por la salinidad como ocurre en la composición isotópica del oxígeno en las conchas de los foraminíferos planctónicos. La amplia distribución de las alquenonas en todo el océano (Marlowe et al., 1990), la fuerte señal de la temperatura derivada de las alquenonas en los sedimentos (Grimalt et al., 2000), su continuidad a través del registro estratigráfico, su resistencia a la degradación aún en sedimentos afectados por la disolución del carbonato (Brassell et al., 1986; Brassell et al., 1987; Marlowe et al., 1990) hacen que las alquenonas sean una valiosa herramienta en estudios paleoceanográficos que pueden extenderse a lo largo de los últimos 45 Ma a través de la contribución de algún ancestro relacionado con los gephyrocapsidos (Marlowe et al., 1990).

\section{Referencias}

Ahagon, N., Tananka, Y., Ujiié, H. 1993. Florisphaera profunda, a possible nannoplankton indicator of Late Quaternary changes in sea-water turbidity at the northwestern margin of the Pacific. Marine Micropaleontology 22, 255-273. 
Asper, V.L. 1987. Measuring the flux and sinking speed of marine snow aggregates. Deep-Sea Research $34,1-17$.

Aubry, M.-P. 1989. Handbook of Cenozoic calcareous nannoplankton, Book 3: Ortholithae (Pentalits, and others), Heliolithae (Fasciculiths, Sphenoliths and others). Micropaleontology Press, American Museum of Natural History, New York.

Balestra, B., Ziveri, P., Monechi, S., Troelstra, S. 2004. Coccolithophorids from the southeast Greenland margin (Northern North Atlantic): production, ecology and the surface sediment record. Micropaleontology 50, 23-24. doi : 10.2113/50. Suppl_1.23.

Baumann, K.-H., Andruleit, H.A., Samtleben, C. 2000. Coccolithophores in the Nordic Seas: comparison of living communities with surface sediment assemblages. Deep-Sea Research II 47, 1743-1772.

Baumann, K.-H., Freitag, T. 2004. Pleistocene fluctuations in the northern Benguela Current system as revealed by coccolith assemblages. Marine Micropaleontology 52, 195-215.

Baumann, K.-H., Andruleit, H., Böeckel, B., Geisen, M., Kinkel, H. 2005. The significance of extant coccolithophores as indicators of ocean water masses, surface water temperature, and paleoproductivity: a review. Paläontologishce Zeitschrift 79, 93-112.

Beaufort, L., Lancelot, Y. Camberlin, P., Cayre, O., Vincent, E., Bassinot, F., Labeyrie, L. 1997. Insolation cycles as a major control of Equatorial Indian Ocean primary production. Science 278, 14511454.

Berger, W.H. 1968. Planktonic foraminifera: selective solution and paleoclimatic interpretation. Deep-Sea Research 15, 31-43.

Billard, C., Inouye, I. 2004. What is new in coccolithophore biology? In: Thierstein, H.R., Young, Y.R. (Eds.), Coccolithophores - From Molecular Processes to Global Impact. Springer-Verlag, Berlin, pp. 1-29.

Bollmann, J., Henderiks, J., Brabec, B. 2002. Global calibration of Gephyrocapsa coccolith abundance in Holocene sediments for paleotemperature assessment. Paleoceanography 17, 1035. doi: 10.1029/2001PA000742.

Boon, J.J., van der Meer, F.W., Schuyl, J.W., de Leeuw, J.W., Schenck, P.A., Burlingame, A.L. 1978. Organic geochemical analyses of core samples from Site 362, Walvis Ridge, Proc. DSDP, Init. Repts., 40. Deep Sea Drilling Program. Government Printing Office, 627-637.

Bown, P.R., Lees, J.A., Young, J.R. 2004. Calcareous nannoplankton evolution and diversity through time. ? In: Thierstein, H.R., Young, Y.R. (Eds.), Coccolithophores - From Molecular Processes to Global Impact. Springer-Verlag, Berlin, pp. 481-508.

Bramlette, M.N. 1961. Pelagic sediments. In: Sears, M. (Ed.), Oceanography, Publication of American Association for the Advancement of Science 67, 345-366.

Brand, L.E. 1994. Physiological ecology of marine coccolithophores. In: Winter, A., Siesser, W.G. (Eds.). Coccolithophores, Cambridge University Press, Cambridge, pp. 39-62.

Brassell, S.C., Eglinton, G., Marlowe, I.T., Pflaumann, U., Sarnthein, M. 1986. Molecular stratigraphy: a new tool for climatic assessment. Nature 320, 129-133.

Brassell, S.C., Eglinton, G., Howell, V.J. 1987. Palaeoenvironmental assessment for marine organic-rich sediments using molecular organic geochemistry. In: Fleet, A.J., Brooks, J. (Eds.), Marine Petroleum Source Rocks. Blackwell, London, pp. 79-98.

Broecker, W.S., Peng, T.-H. 1992. Tracers in the sea. Eldigio Press, Palisiades, New York.

Brown, J., Colling, A., Park, D., Phillips, J., Rothery, D., Wright, J. 1989. Ocean chemistry and deep-sea sediments. Oxford (Pergamon Press). $134 \mathrm{p}$.

Brown, E., Colling, A., James, R., Park, D., Phillips, J., Rothery, D., Wright, J. 2005. Marine Biogeochemical cycles. Oxford (Pergamon Press). $130 p$

Cavalier-Smith, T. 1998. A revised six-kingdom system of life. Biological Reviews of the Cambridge Philosophical Society, 73: 203-266.

Colmenero-Hidalgo, E., Flores, J.-A., Sierro, F.J. 2002. Biometry of Emiliania huxleyi and its biostratigraphic significance in the eastern north Atlantic Ocean and Western Mediterranean Sea in the last 20,000 years. Marine Micropaleontology 46, 247- 263.

Conte, M.H., Volkmann, J.K., Eglinton, G. 1994. Lipid biomarkers of Haptophyta. In Green, J.C., Leadbeater, B.S.C. (Eds.), The Haptophyte Algae 51, The Haptophyte Algae. Systematics Association Special Volume 51. Clarendon Press, Oxford. pp. 351-377. 
Cros, L., Fortuño, J.-M. 2002. Atlas of Northwestern Mediterranean coccolithophores. Scientia Marina 66 (Suppl. 1). Institut de Ciències del Mar, CMIMA-CSIC Barcelona. pp. 1-194.

de Vargas, C., Aubry, M-P., Probert, I., Young, J. 2007. Origin and evolution of coccolithophores: From coastal hunters to oceanic farmers. In: Falkowsko, P., Knoll, A., Evolution of Primary Producers in the Sea. ELSEVIER, Academic Press. Amsterdam, 251-285.

Dittert , N., Baumman, K.H., Bickert, T., Heinrich, R., Huber, R., Kinkel, H.. Meggers, H. 1999. Carbonate dissolution in the deep-sea: methods, quantification, and paleoceaographic application. In: Fischer, G., Wefer, G. (Eds.) Use of proxies in Paleoceanography: Examples from the South Atlantic. Springer-Verlag, Berlin. p. 255-284.

Edvardsen, B., Eikrem, W., Green, J.C., Andersen, R.A., Staay van der, S.Y.M., Medlin, L.K. 2000. Phylogenetic reconstructions of the Haptophyta inferred from $18 \mathrm{~S}$ ribosomal DNA sequences and available morphological data. Phycology, 39, 19-35.

Eppley, R.W., Peterson, B.J. 1979. Particulate organic matter flux and planktonic new production in the deep ocean. Nature 282, 677-680. doi: 10.1038/282677a0.

Erez, J. 2003. The source of ions for biomineralization in foraminifera and their applications for paleoceanographic proxies. In Dove, P. M., de Yoreo, J. J., Weiner, S., Biomineralization. Reviews in Mineralogy and Geochemistry 54, 115-149.

Feely, R., Sabine, C., Takahashi, T., Wanninkhof, R. 2001. Uptake and storage of carbon dioxide in the ocean: the global CO2 survey. Oceanography 14, 18 -32.

Farrell, J.W:, Prell, W. 1989. Climatic change and CaCo3 preservation: an 800,000 year bathymetric reconstruction from the Central Equatorial Pacific Ocean. Paleoceanography 4, 447-466.

Flores, J.-A., Sierro, F.J. 2007. Coccoliths. In Scott, A.E., Quaternary Science, ELSEVIER, Academic Press, Amsterdam, pp. 1634-1646.

Flores, J.A., Bárcena, M.A., Sierro, F.J. 2000. Ocean-surface and wind dynamics in the Atlantic Ocean off Northwest Africa during the last 140000 years. Palaeogeography, Palaeoclimatology, Palaeoecology 161, 459-478.

Geisen, M., Billard, C., Broerse, A.T.C., Cros, L., Probert, I., Young, J.R. 2002. Life-cycle associations involving pairs of holococcolithophorid species: Intraspecific variation or cryptic speciation? European Journal of Phycology 37, 531-550.

Grimalt, J.O., Rullkötter, J. Sicre, M.-A., Summons, R., Farrington, J., Harvey, H.R., Goñi, M., Sawada, K. 2000. Modifications of the $C_{37}$ alkenone and alkenoate composition in the water column and sediment: Possible implications for sea surface temperature estimates in paleoceanography. Geochemistry Geophysics Geosystems 1. doi : 2000GC000053.

Heinrich, R., Baumman, K.H. Gerhardt., S., Gröger, M., Volbers, A. 2004. Carbonate preservation in deep and intermediate waters in the South Atlantic: evaluation and geologic record (a review). In: Wefer, G., Mulitza, S., Ratmeyer, V. (Eds.) The South Atlantic in the Late Quaternary Reconstruction of material budget and current systems. Springer-Verlag, Berlin. p. 645-670.

Hendericks, J., Bollmann, J. 2004. The Gephyrocapsa sea surface palaeothermometer put to the test: comparison with alkenone and foraminifera proxies off NW Africa. Marine Micropaleontology 50, 161-184.

Hibberd, D.J. 1976. The ultrastructure and taxonomy of the Chrysophyceae (Haptophyceae): survey with some new observations on the ultrastructure of the Chrysophyceae. Bot. J. Linn. Society 72, 5580.

Holligan, P.M., Viollier, M., Harbour, D.S., Camus, P., Champagne-Phillippe, M. 1983. Satellite and ship studies of coccolithophore production along a continental shelf edge. Nature 304, 339-342.

Honjo, S. 1975. Dissolution of suspended coccoliths in the deep-sea water column and sedimentation of coccolith ooze. In: Sliter, W.B., Be, A.W.H., Berger, W.H. (Eds.), Dissolution of deep-sea carbonates, Cushman Found, Foraminiferal Research Special Publication 13, 115-128.

Honjo, S. 1976. Coccoliths: production, transportation and sedimentation. Marine Micropaleontology 1, 6579.

Honjo, S., Manganini, S.J., Cole, J.J. 1982. Sedimentation of biogenic matter in the deep ocean. DeepSea Research 29, 609-625.

Honjo, S., Doherty, K.W., Agrawal, Y.C., Asper, V.L. 1984. Direct optical assessment of large amorphous aggregates (marine snow) in the deep ocean. Deep-Sea Research 31, 67-76. 
Jordan, R.W., Chamberlain, AHL. 1997. Biodiversity among haptophyte algae. Biodiversity and Conservation 6, 131-152.

Jordan, R.W., Kleijne, A., Heimdal, B.R., Green, J.C. 1995. A glossary of the extant Haptophyta of the world. Journal of the Marine Biological Association of the United Kingdom 75, 769-814.

Kameo, K., Shearer, M.C., Droxler, A.W., Mita, I., Watanabe, R., Sato, T. 2004. Glacial-interglacial surface water variations in the Caribbean Sea during the last $300 \mathrm{ky}$ based on calcareous nannofossil analysis. Palaeogeography, Palaeoclimatology, Palaeoecology 212, 65-76.

Knappertsbusch, M., Cortes, M.Y., Thierstein, H.R. 1997. Morphologic variability of the coccolithophorid Calcidiscus leptoporus in the plankton, surface sediments and from the Early Pleistocene. Marine Micropaleontology 30, 293-317.

Knauer, G.A., Martin, J.H., Karl, D.M. 1984. The flux of particulate organic matter out of the euphotic zone. Proceedings of Workshop on the Global Ocean Flux Study. National Academy Press, Washington, DC, pp. 136-150.

Kinkel, H., Baumann, K.-H., Čepek, M. 2000. Coccolithophores in the equatorial Atlantic Ocean: response to seasonal and Late Quaternary surface water variability. Marine Micropaleontology 39, 87-112.

Klöcker, R., Ganssen, G., Jung, S. J. A., Kroon, D., Henrich, R. 2006. Late Quaternary millenial-sclae variability in pelagic aragonite preservation off Somalia. Marine Micropaleontology 59, 171-183.

Marchant, H.J., Thomsen, H.A. 1994. Haptophytes in polar waters. In: Green, J.C., Leadbeater, B.S.C. (Eds.), The Haptophyte Algae. Systematics Association Special Volume 51. Clarendon Press, Oxford. pp. 187-208.

Marlowe, I.T., Brassell., S.C., Eglinton, G., Green, J.C. 1984. Long chain unsaturated ketones and esters in living algae and marine sediments. Organic Geochemistry 6, 135-141.

Marlowe, I.T., Brassell, S.C., Eglinton, G., Green, J.C. 1990. Long-chain alkenones and alkyl alkenoates and the fossil coccolith record of marine sediments. Chemical Geology 88, 349-375.

Martínez, I., Rincon, D., Yokoyama, Y., Barrows, T. 2005. Foraminifera and coccolithophorid assemblage changes in the Panama Basin during the last deglaciation: Response to sea-surface productivity induced by a transient climate change. Palaeogeography, Palaeoclimatology, Palaeoecology 234, 114-126.

McIntyre, A., Bé, A.W.H. 1967. Modern coccolithophoraceae of the Atlantic Ocean: I. Placoliths and cyrtoliths. Deep-Sea Research I 14, 561-597.

McIntyre, A., Bé, A., Roche, M. 1970. Modern Pacific Coccolithophorida: a peleontological thermometer. Transactions of the New York Academy of Science II 32/6. pp. 720-731.

Milliman, J.D. 1993. Production and accumulation of calcium carbonate in the ocean: budget of a nonsteady state. Global Biogeochemical Cycles 7:927-957.

Molfino, B., Mclntyre, A. 1990a. Precessional forcing of nutricline dynamics in the equatorial Atlantic. Science 249, 766-769.

Molfino, B., Mclntyre, A. 1990b. Nutricline variation in the equatorial Atlantic coinciding with the Younger Dryas. Paleoceanography 5, 997-1008

Narciso, A., Cachão, M., de Abreu, L. 2006. Coccolithus pelagicus subsp. pelagicus versus Coccolithus pelagicus subsp. braarudii (Coccolithophore, Haptophyta): A proxy for surface subartic Atlantic waters off Iberia during the last 200 kyr. Marine Micropaleontology 59, 15-34.

Okada, H., Honjo, S. 1973. The distribution of oceanic coccolithophorids in the Pacific. Deep-Sea Research 20, 355-374.

Okada, H., Mclntyre, A. 1977. Modern coccolithophores of the Pacific and North Atlantic Oceans. Micropaleontology 23, 1-55.

Okada, H., Matsuoka, M. 1996. Lower-photic nannoflora as an indicator of the late Quaternary monsoonal paleo-record in the tropical Indian Ocean. In: Moguilevsky, A., Whatley, A.M. (Eds.), Microfossils and Oceanic Environments, Aberystwyth Press, Aberystwyth. pp. 231-245.

Parente, A., Cachão, M., Baumann, K.-H., de Abreu, L., Ferreira, J. 2004. Morphometry of Coccolithus pelagicus s.l. (Coccolithophore, Haptophyta) from offshore Portugal, during the last $200 \mathrm{kyr}$. Micropaleontology 50 (supplement), 107-120.

Perch-Nielsen, K. 1985a. Mesozoic calcareous nannofossils. In: Bolli, H.M., Saunders, J.B., PerchNielsen, K. (Eds.), Plankton Stratigraphy. Cambridge University Press, Cambridge. pp. 329-426.

Perch-Nielsen, K. 1985b. Cenozoic calcareous nannofossils In: H.M. Bolli, J.B. Saunders and K. PerchNielsen (Eds.), Plankton Stratigraphy. Cambridge University Press. Cambridge. pp. 427-555. 
Pienaar, R. 1994. Ultrastructure and calcification of coccolithophores. In: Winter, A., Siesser, W.G. (Eds.). Coccolithophores, Cambridge University Press, Cambridge, pp. 13-37.

Prahl, F.G., Wakeham, S.G. 1987. Calibration of unsaturation patterns in long-chain ketone compositions for palaeotemperature assessment. Nature 330: 367-369.

Rickaby, R.E.M., Bard, E., Sonzogni, C., Rostek, F., Beaufort, L., Barker, S., Rees, G., Schrag, D.P. 2007. Coccolith chemistry reveals secular variations in the global ocean carbon cycle? Earth and Planetary Science Letters 253, 83-95.

Ridgwell, A., Zeebe, R.E. 2005. The role of the global carbonate cycle in the regulation and evolution of the Earth system. Earth and Planetary Science Letters 234, 299-315.

Saavedra-Pellitero, M. 2006. Distribución biogeográfica de las asociaciones de cocolitóforos en el Pacífico ecuatorial y suroriental. Tesina. Universidad de Salamanca, Salamanca, 137p.

Sáez, A.G., Probert, I., Young, J.R., Edvarsen, B., Eikrem, W., Medlin, L.K. 2004. A review of the phylogeny of the Haptophyta. In: Thierstein, H.R., Young, Y.R. (Eds.), Coccolithophores - From Molecular Processes to Global Impact. Springer-Verlag, Berlin, pp. 251-269.

Samtleben, C., SchroK der, A. 1992. Living coccolithophore communities in the Norwegian-Greenland Sea and their record in sediments. Marine Micropaleontology 19, 333-354.

Samtleben, C., Shafer, P., Andruleit, H., Baumann, A., Baumann, K.H., Kohly, A., Matthiessen, J., Scholder-Ritzrau, A. 1995. Plankton in the Norwegian-Greenland Sea: from living communities to sediment assemblages - an actualistic approach. Geologische Rundschau 84, 108-136.

Schlesinger, W. H. 2000. Biogeoquímica. Un análisis del cambio global. Ariel S.A. Barcelona, Spain, 593 $\mathrm{pp}$.

Shanks, A.L., Trent , J.D. 1980. Marine snow: sinking rates and potential role in vertical flux. Deep-Sea Research 27, 137-143.

Silver, M.W., Shanks, A.L., Trent, J.D. 1978. Marine snow: microplankton habitat and source of smallscale patchiness in pelagic populations. Science 201, 371-373.

Steinmetz, J.C. 1994. Sedimentation of coccolithophores. In: Winter, A., Siesser, W.G. (Eds.). Coccolithophores, Cambridge University Press, Cambridge, pp. 179-197.

Sprengel, C., Baumann, K.-H., Henderiks, J., Henrich, R., Neuer, S. 2002. Modern coccolithophore and carbonate sedimentation along a productivity gradient in the Canary Islands region: seasonal export production and surface accumulation rates. Deep-Sea Research II 49, 3577-3598.

Stoll, H., Ziveri, P. 2004. Coccolithophorid-based geochemical paleoproxies. In: Thierstein, H.R., Young, Y.R. (Eds.), Coccolithophores - From Molecular Processes to Global Impact. Springer-Verlag, Berlin, pp. 529-562.

Stoll, H.M., Arevalos, A., Burke, A., Ziveri, P., Mortyn, G., Shimizu, N., Unger, D. 2007. Seasonal cycles in biogenic production and export in Northern Bay of Bengal sediment traps. Deep-Sea Research II $54,558-580$.

Takahashi, K., Okada, H. 2000. The paleoceanography for the last 30,000 years in the southeastern Indian Ocean by means of calcareous nannofossils. Marine Micropaleontology 40, 83-103.

Tappan, H. 1980. Haptophyta, coccolithophores, and other calcareous nannoplankton. In: H. Tappan (Ed.), The Paleobiology of plant protists, Freeman, California. pp. 678-803.

Van Cappellen, P. 2003. Biomineralization and global biogeochemical cycles. . In Dove, P. M., de Yoreo, J. J., Weiner, S., Biomineralization. Reviews in Mineralogy and Geochemistry 54, 357-381.

Volkman J. K., Eglinton G., Corner E. D. S., Forsberg T. E. V. 1980a. Long-chain alkenes and alkenones in the marine coccolithophorid Emiliania huxleyi. Phytochemistrv 19, 2619-2622.

Volkman J. K., Eglinton G., Corner E. D. S., Sargent J. R. 1980b. Novel unsaturated straight-chain $\mathrm{C}_{37}-\mathrm{C}_{39}$ methyl and ethyl ketones in marine sediments and a coccolithophore Emiliania huxleyi. In Douglas A. G., Maxwell J. R. (Eds.), Advances in Organic Geochemistry 1979, Pergamon Press, Oxford. pp. 219-228.

Volkman, J.K., Barrett, S.M., Blackburn, S.I., Sikes, E.L. 1995. Alkenones in Gephyrocapsa oceanica; implications for studies of paleoclimate. Geochemica et Cosmochemica Acta 59, 513-520.

Weaver, P.P.E., Pujol, C. 1988. History of the last deglaciation in the Alboran Sea (western Mediterranean) and adjacent north Atlantic as revealed by coccolith floras. Palaeogeography, Palaeoclimatology, Palaeoecology 64: 35-46. 
Winter, A., Jordan, R. W., Roth, P.H. 1994. Biogeography of living coccolithophores in ocean waters. In: Winter, A., Siesser, W.G. (Eds.). Coccolithophores, Cambridge University Press, Cambridge, pp. 161-177.

Young, J.R. 1994. Functions of coccoliths. In: Winter, A., Siesser, W.G. (Eds.). Coccolithophores, Cambridge University Press, Cambridge, pp. 63-82.

Young, J.R., P.R. Bown. 1997a. Higher classification of calcareous nannofossils. Journal of Nannoplankton Research, 19: 15-20.

Young, J.R., Bown. 1997b. Cenozoic calcareous nannoplankton classification. Journal of Nannoplankton Research, 19: 36-47.

Young, J.R., Ziveri, P. 2000. Calculation of coccolith volume and its use in calibration of carbonate flux estimates. Deep-Sea Research A 47, 1679-1700.

Young, J.R., Henriksen, K. 2003. Biomineralization within vesicles: the calcite of coccoliths. In Dove, P. M., de Yoreo, J. J., Weiner, S., Biomineralization. Reviews in Mineralogy and Geochemistry 54, 189-215.

Young, J.R., Bergen, J.A., Bown, P.R., Burnett, J.A., Fiorentino, A., Jordan, R.W., Kleijne, A., Van Niel B.E., Romein, AJT., von Salis K. 1997. Guidelines for coccolith and calcareous nannofossil terminology. Palaeontology 40, 875-912.

Young, J., Geisen, M., Cros, L., Kleijne, A., Sprengel, C., Probert, I., Østergaard, J. 2003. A guide to extant coccolithophore taxonomy. Journal of Nannoplankton Research, Special Issue 1, 125p.

Ziveri, P., Rutten, A., de Lange, G.J., Thomson, J., Corselli, C. 2000. Present-day coccolith fluxes in central eastern Mediterranean sediment traps and surface sediments. Palaeogeography, Palaeoclimatology, Palaeoecology 158, 175-195.

Ziveri, P., Baumann, K.-H., Böeckel, B., Bollmann, J., Young, J.R. 2004. Biogeography of selected Holocene coccoliths in the Atlantic Ocean. In: Thierstein, H.R., Young, Y.R. (Eds.), Coccolithophores - From Molecular Processes to Global Impact. Springer-Verlag, Berlin, pp. 403428. 


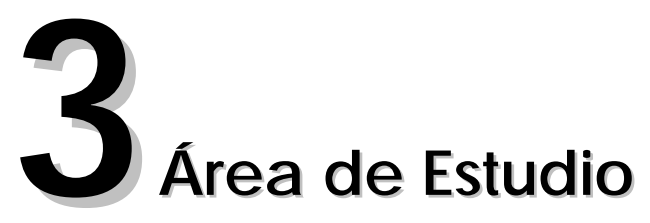

3.1. Océano Pacífico oriental tropical: cuenca de Panamá

3.1.1. Escenario tectónico

3.1.2. Oceanografía

Dinámica superficial y subsuperficial

Dinámica intermedia y profunda

3.2. Océano Atlántico occidental tropical: cuenca de la Guayana

3.2.1. Escenario tectónico

3.2.2. Oceanografía

Dinámica superficial y subsuperficial

Dinámica intermedia y profunda

3.3. Los Andes: Geología y clima

Referencias 


\subsection{Océano Pacífico oriental tropical: cuenca de Panamá}

\subsubsection{Escenario tectónico}

Los testigos oceánicos ODP (“Ocean Drilling Program”) 1240, 1241 y 1242 localizados en la cuenca de Panamá en el océano Pacífico oriental tropical norte, están situados en dos placas oceánicas diferentes: el testigo ecuatorial (1240) sobre la placa Nazca y los testigos tropicales (1241 y 1242) sobre la placa Cocos. La cuenca está definida como el área que circundan las cordilleras asísmicas Cocos y Carniege al oeste y sur respectivamente; y los márgenes continentales activos de Colombia y Ecuador al este, y de Panamá y Costa Rica al norte. La formación de la cuenca data del Mioceno ( 22.7 Ma) por el centro de expansión de Galápagos (que separa las placas Cocos, Nazca y la Dorsal Este del Pacífico ("East Pacific Rise"), después de la rotura de la Placa Farallón, que hizo que la placa tectónica de Cocos subdujera hacia el norte y la placa de Nazca hacia el este (Lonsdale y Klitgord, 1978; Pennington, 1989) bajo la placa Suramericana (Meissner et al., 1976). Algunos autores han datado nuevamente el inicio de este centro de expansión entorno a unos 19.5 Ma (Barckhausen et al., 2001) (Figs. 3.1, 3.1).

La historia geológica del centro de expansión Cocos-Nazca está determinada por su expansión asimétrica a velocidades intermedias (tasa promedio de 60-70 mm/año), por la formación de pequeños montículos en los límites de las placas, y la influencia del "punto caliente" ("Hotspot") de Galápagos situado actualmente a $\sim 170 \mathrm{Km}$ al sur del centro de expansión (Canales et al., 1997; Hauff et al., 1997).

Hoy en día se acepta que las dorsales de Cocos y Carniege se hayan formado durante la interacción entre el centro de expansión y el punto caliente. La Dorsal de Carniege está creciendo al oeste, alrededor de las islas Galápagos. Los lugares más antiguos de la trayectoria del punto caliente de Galápagos están localizados en la placa de Cocos cerca de Costa Rica, haciendo parte de la Dorsal de Cocos y su edad corresponde entre unos 13-14.5 Ma (Werner et al., 1999; Hoernle et al., 2000). Los datos geoquímicos de los complejos oceánicos acrecionados a Costa Rica indican que el punto caliente estuvo activo por lo menos $90 \mathrm{Ma}$ (Canales et al., 1997; Hauff et al., 1997). 


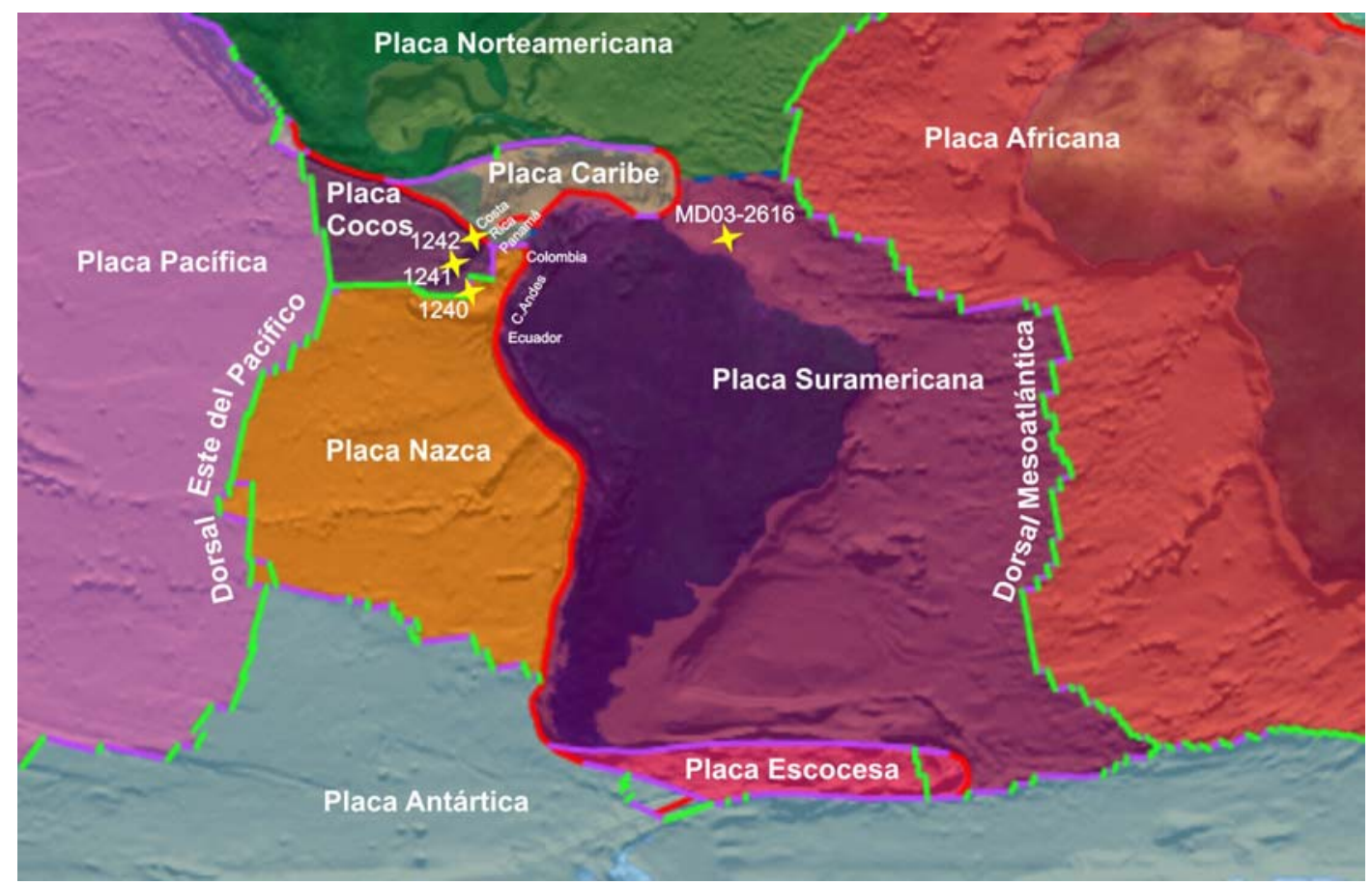

Fig. 3.1. Representación de las placas tectónicas Nazca, Cocos y Suramericana sobre las que se localizan los testigos 1240, 1241, 1242 y MD03-2616, y su relación con las placas vecinas. Los márgenes verdes corresponden a márgenes convergentes, los márgenes rojos a márgenes divergentes y los márgenes morados a márgenes en donde su geometría y naturaleza tectónica son muy complejas. Fuente NASA/Goddard Space Flight Center Scientific Visualization Studio (http://visibleearth.nasa.gov/)

El fondo oceánico en la cuenca de Panamá está caracterizado por una topografía rugosa y por anomalías magnéticas de gran amplitud, en contraste con la Dorsal Este del Pacífico ("East Pacific Rise") que se formó durante una expansión rápida y que se caracteriza por una topografía suave y anomalías magnéticas de baja amplitud (Hey, 1977, Wilson and Hey, 1995, Barckhausen et al., 2001).

Los sedimentos del testigo 1240 forman parte de un valle abisal del centro de expansión oceánica en la Dorsal de Carniege, con variaciones del relieve entre 200 y 300 m (Fig. 3.2). La corteza oceánica basáltica fue generada a través del centro de expansión hace $3 \mathrm{Ma}$ (Hey et al., 1977). Este valle abisal actúa como una trampa de sedimentos pelágicos que provienen del sistema de surgencia ecuatorial, dando lugar a una cobertera pelágica de 250 a $280 \mathrm{~m}$ de espesor en un tiempo muy breve (<3 Ma). Los sedimentos del testigo 1241 forman parte de una rampa de sedimentos de pendiente suave en el flanco norte de la cadena Cocos, en medio de una topografía suave de salientes volcánicos que pueden levantarse pocos cientos de metros sobre la rampa (Fig. 3.2). La cobertera pelágica de entre 400 y $500 \mathrm{~m}$ de espesor, se adelgaza hacia el sur y presenta canales fósiles como signo de que ha ocurrido transporte de sedimentos. La corteza oceánica de edad entre 11-13 Ma y la Dorsal se han formado por vulcanismo de 
punto caliente. El testigo ODP 1242 está ubicado en un graben en la cresta de la Dorsal de Cocos (Fig. 3.2), en una cuenca subsidente, pequeña, con $460 \mathrm{~m}$ de espesor de sedimentos pelágicos, en medio de una geometría estructural muy compleja afectada por fallamiento.

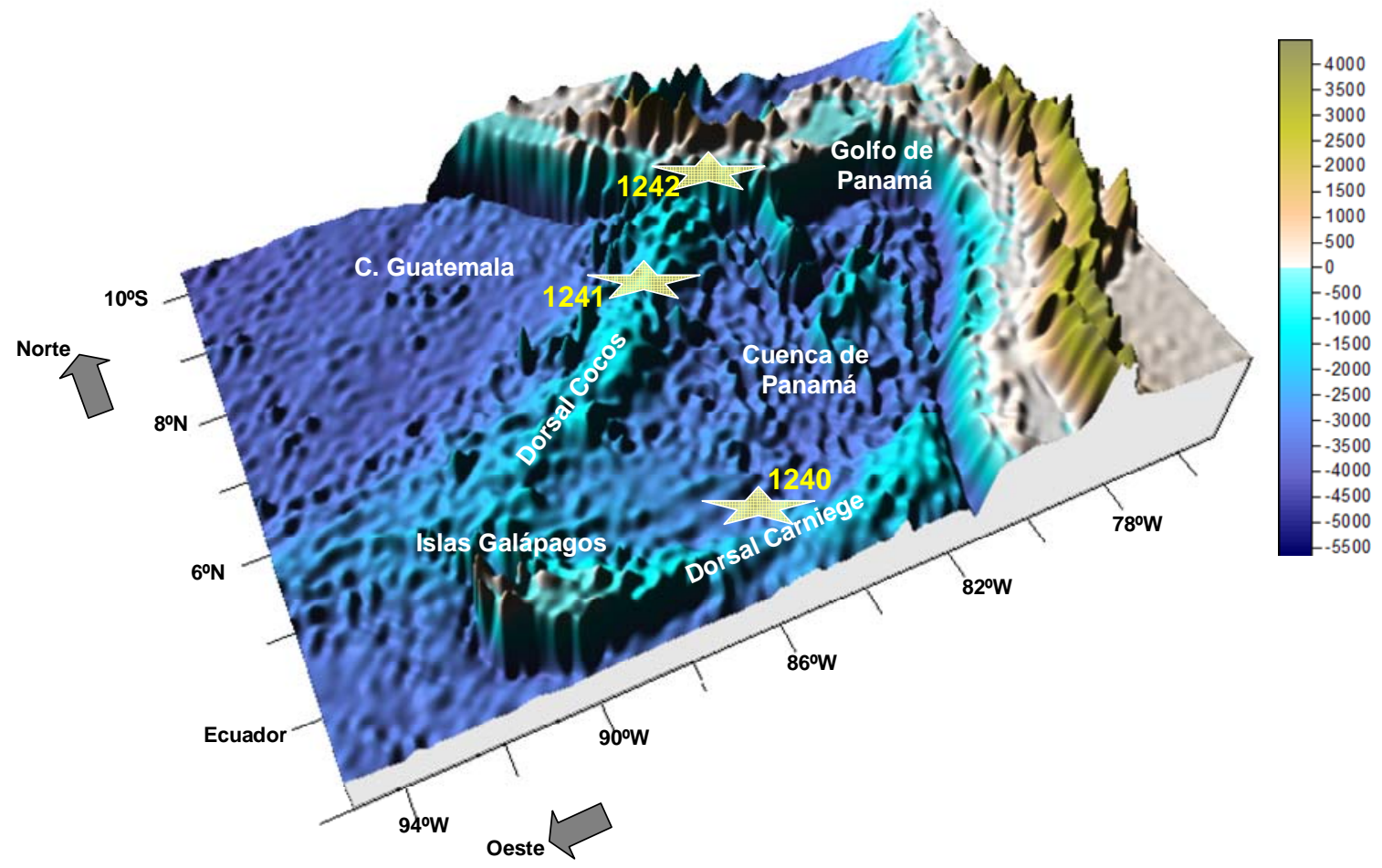

Fig. 3.2. Localización 3D de los testigos 1240, 1241 y 1242 en la cuenca de Panamá (Pacífico oriental tropical) y las variaciones del relieve en la cuenca. La escala de colores resalta las principales características batimétricas. Tomada de la base de datos topográfica de Smith y Sandwell (1997).

La sedimentación en estos testigos ha estado sujeta a los principales procesos tectónicos asociados a la evolución del estrecho de Drake (29-23 Ma); al levantamiento de los Andes (desde el Oligoceno tardío al Plioceno; Warne et al., 2002); a los movimientos de las placas Pacífica, Nazca y Cocos; y al cierre del Istmo de Panamá entre los 13 y $2.7 \mathrm{Ma}$, causando efectos importantes en la paleoclimatología y paleoceanografía regional y global, tales como la influencia de los alisios en las corrientes superficiales del océano Pacífico y la intensificación de la glaciación en los hemisferios Norte y Sur durante el Plioceno (Mix et al., 2003; Tiedemann y Mix, 2007).

\subsubsection{Oceanografía}

\section{Dinámica superficial y subsuperficial}

La principal característica en el patrón de circulación en el océano Pacífico tropical oriental, es la alineación asimétrica de las corrientes oceánicas al norte y sur del ecuador y la localización de la 
zona de convergencia de los vientos alisios (llamada Zona de Convergencia Intertropical "Intertropical Convergence Zone", ITCZ) siempre en el hemisferio norte debido a la interacción entre el océano, la atmósfera y el continente (Philander, 1996). Aunque los tres testigos estudiados en el Pacífico se ubican a la misma cuenca, cada uno está bañado por masas de agua de origen diferente (Fig. 3.3).

Las corrientes superficiales que entran al océano Pacífico oriental ecuatorial incluyen las corrientes de Perú ("Peru Current", PC) y California (“California Current”, CCa). Estas corrientes arrastran aguas frías y salinas desde las altas latitudes al sur y norte respectivamente, hacia el ecuador. Cuando ambas corrientes llegan al Pacífico ecuatorial toman la dirección de los vientos alisios, este-oeste, y forman las corrientes Surecuatorial ("South Equatorial Current", SEC) y Norecuatorial ("North Equatorial Current", NEC). Otra de las corrientes más importantes en el Pacífico ecuatorial es la corriente Subsuperficial Ecuatorial (“Equatorial Undercurrent”, EUC), que fluye en dirección oeste-este debajo de la SEC. Estas dos últimas corrientes, SEC y EUC, alimentan la divergencia ecuatorial del Pacífico oriental con aguas frías, relativamente salinas, ricas en nutrientes, que dan lugar a la Lengua Fría Ecuatorial ("Equatorial Cold Tongue”, ECT) (Wyrtki, 1981). La ECT está caracterizada oceanográficamente por temperaturas más frías de lo que corresponde a su posición geográfica y está controlada por la intensidad de los vientos alisios y la variabilidad de la célula de surgencia equatorial (Wyrtki, 1981). Otras corrientes subsuperficiales están asociadas al Domo de Costa Rica, causando turbulencia profunda y surgencia local de las aguas (Hofmann et al., 1981).

El Frente Ecuatorial ("Equatorial Front”, EF) separa las aguas frías y relativamente salinas de la ECT de las aguas tropicales cálidas y menos salinas (más conocidas en la literatura como "warm pool") (Pak y Zaneveld, 1974; Wyrtki, 1981). La cuenca de Panamá, al norte del ecuador, se caracteriza por altas temperaturas y baja salinidad del agua superficial $\left(>30^{\circ} \mathrm{C}\right.$; $\sim 32 \%$ ) y una picnoclina somera y bien definida (centrada en los 20-40 m de profundidad). Estas características reflejan un predominio de la precipitación sobre la evaporación (Magaña et al., 1999), que da lugar a la estabilidad vertical de la columna de agua. La precipitación en el océano Pacífico oriental está controlada por el transporte de agua evaporada que viene del Caribe y del Atlántico (Jousaumme et al., 1986), permitiendo que las aguas de la cuenca de Panamá al norte de ecuador, sean relativamente menos salinas, y las aguas del Atlántico sean relativamente más salinas. Este transporte de agua juega un papel muy importante en el clima, porque ayuda a mantener la circulación termohalina (Zauker et al., 1994; Rahmstorf, 1995). La dinámica de las 


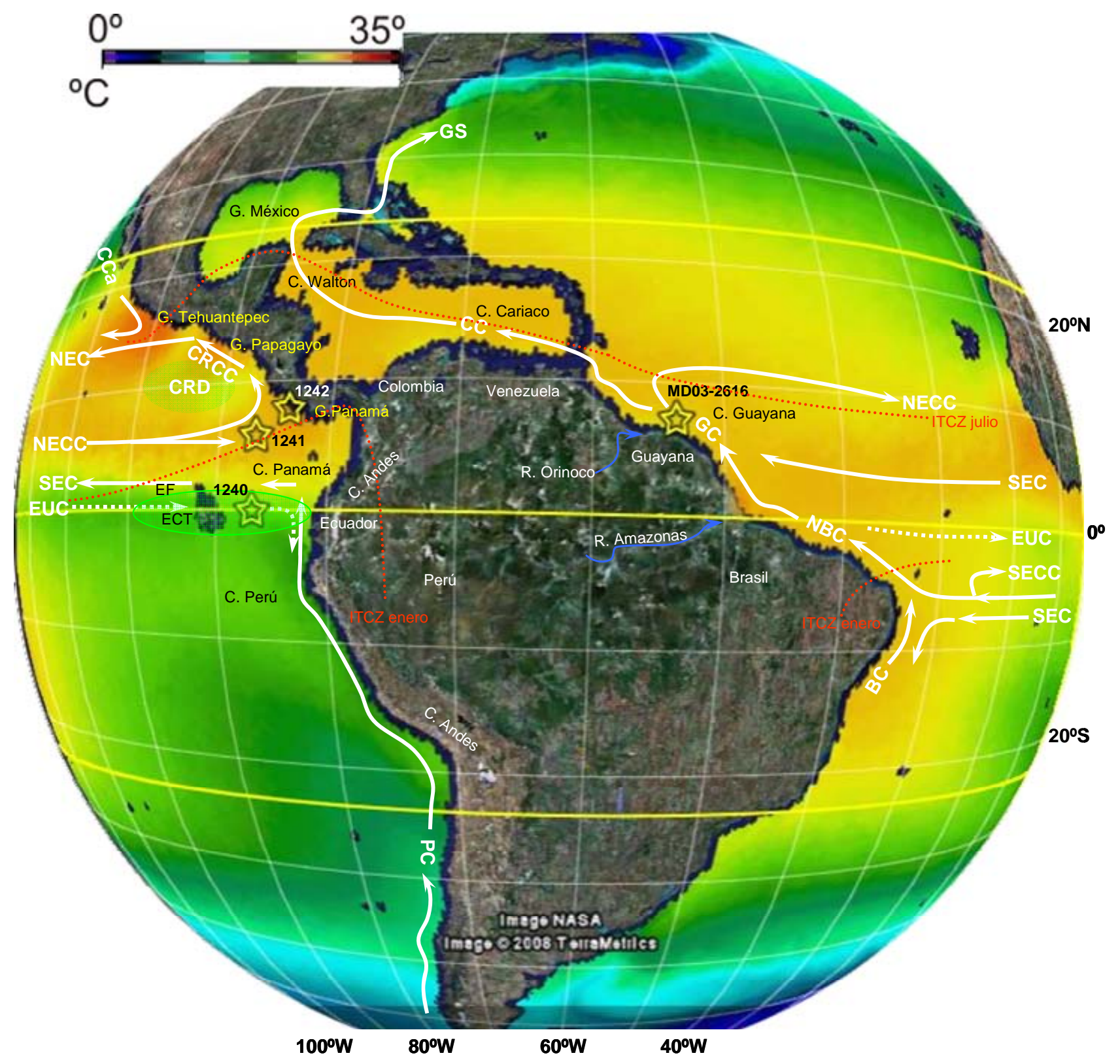

Fig. 3.3. Localización de los testigos estudiados: ODP 1240, 1241 y 1242 en el océano Pacífico oriental tropical, y MD03-2616 en el océano Atlántico occidental tropical. Los patrones de circulación superficial: SEC, corriente Surecuatorial; NEC, corriente Norecuatorial; PC, corriente del Perú; EUC, corriente Subsuperficial; CCa, corriente de California; CRCC, corriente costera de Costa Rica; BC, corriente del Brasil, NBC, corriente Norte del Brasil; CC, corriente del Caribe; GS, corriente del Golfo; EF, Frente Ecuatorial; ECT, Lengua Fría Ecuatorial (Fiedler y Talley, 2006; Kessler, 2006; Richardson et al., 1994). Los patrones de circulación atmosférica: ITCZ, Zona de Convergencia Intertropical durante el verano boreal (ITCZ julio) y el invierno boreal (ITCZ enero) (Haugh et al., 2003). Las corrientes y contracorrientes superficiales están representadas por flechas blancas continuas, y las corrientes subsuperficiales con flechas blancas punteadas. Las principales cuencas $(C)$ sedimentarias y golfos $(G)$ también están localizadas. El mapa de fondo muestra la variación de la temperatura en el océano superficial durante el invierno boreal. Fue tomado de la base de datos de la NASA Earth Observations (neo.sci.gsfc.nasa.gov) y dibujado en el Google Earth. 
aguas en el Pacífico oriental influye en la producción biológica y en el ciclo del carbono, los cuales son controlados por la variación estacional de los vientos alisios que manejan la célula de surgencia ecuatorial, los eventos interanuales-decadales de "El Niño", y las variaciones de la ITCZ (Philander, 1995; Clement et al., 1999; Fedorov y Philander, 2000).

Esta dinámica océano-atmosférica en el Pacífico tropical puede ser debilitada o intensificada por la ocurrencia del fenómeno ENSO, a través de la fase cálida ("El Niño") o a través de su fase fría ("La Niña") respectivamente, repercutiendo en la cadena trófica (tal como se ha introducido en las secciones 1.5 y 1.7 )

\section{Dinámica intermedia y profunda}

Las aguas intermedias de la cuenca de Panamá proceden principalmente del Pacífico Norte, a través de las zonas de fractura de la Dorsal de Cocos (Mix et al., 2003) y una pequeña contribución de las masas de agua intermedias que se originan en el Pacífico sur. De esta manera, las masas de agua intermedias en el Pacífico norte, al sur del Frente Subártico, están caracterizadas por un perfil que registra un mínimo de salinidad, pudiéndose diferenciar 2 tipos de masas de agua de acuerdo con su origen, una de ellas sigue la dirección norte-sur y la otra, la dirección sur-norte (Fig. 3.4). La más somera de las dos es la masa de Agua Intermedia del Pacífico Norte ("North Pacific Intermediate Water", NPIW) fuertemente definida por su mínimo de salinidad entre 34.0 a 34.3 psu ("practical salinity units", unidades prácticas de salinidad) situándose en un rango de salinidad muy estrecho, entre los 26.7 y $26.9 \sigma_{\theta}$, a profundidades comprendidas entre los 300 a 800 m (Talley, 1985; 1993; You, 2003a). La NPIW se forma en la región subpolar del Pacífico noroccidental, con la contribución de las masas de agua frías y poco salinas del Agua Intermedia de Okhotsk (“Okhostk Intermediate Water", OIW) en el mar de Okhotsk y del Agua Intermedia del Golfo de Alaska ("Gulf Alaska Intermediate Water", GAIW; You, 2005). Así, la formación de la NPIW está sujeta a la difusión vertical y a la producción de agua densa durante el invierno en el mar de Okhotsk y en la región subártica debido a que la NPIW no aflora en el océano abierto subpolar (You, 2003a). La contribución de la OIW se establece a lo largo de la zona frontal subártica-tropical ("Subarctic-tropical frontal zone", SATFZ) por procesos de transformación de esta masa de agua, como "cabbeling" que describe su descenso causado por mezcla lateral, y "thermobaricity" que describe el aumento en la densidad por cambios en la temperatura y la presión (You, 2003b; 2005). La contribución de la GAIW se da en menor proporción que la OIW, y se limita a la capa superior de la NPIW, a los períodos de invierno y al giro oriental subtropical (Talley, 1993, You, 2003b; 2005). Durante su recorrido hacia 

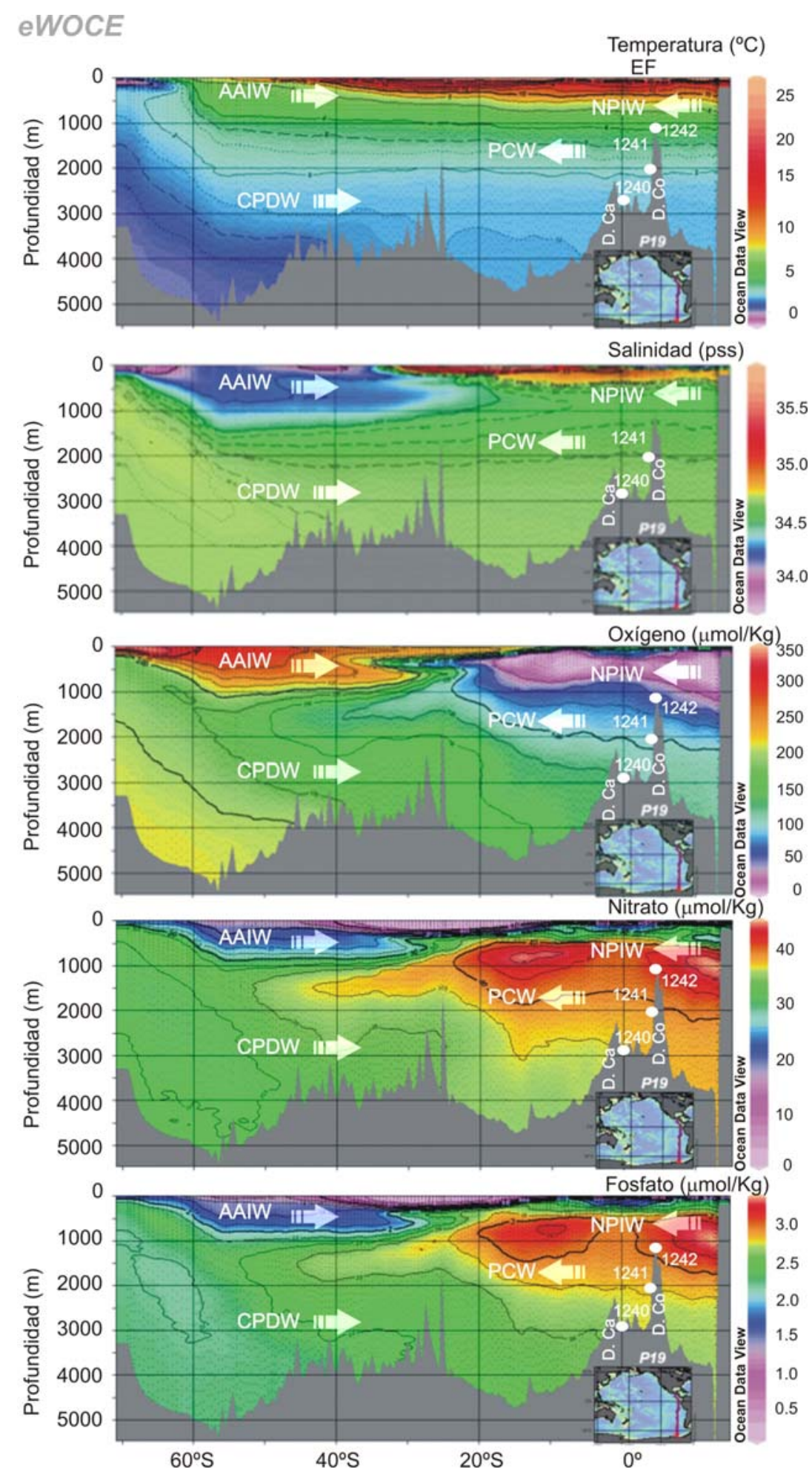

Fig. 3.4. Configuración de las masas de agua intermedias y profundas en el océano Pacífico oriental tropical y algunas de sus propiedades fisico-químicas (temperatura, salinidad, oxígeno, nitrato y fosfato), en el transecto paleoceanográfico meridional P19 tomada del eWOCE (World Ocean Circulation Experiment; (Schlitzer, 2000). AAIW, Agua Antarctica Intermedia; NPIW, Agua Intermedia del Pacífico Norte; PCW, Agua Central del Pacífico; CPDW, Agua Central Profunda del Pacífico; EF, Frente Ecuatorial; D.Co, Dorsal de Cocos; D.Ca, Dorsal de Carniege. 
el sur, la NPIW se caracteriza por su escasa interacción con la atmósfera, lo cual hace que su salinidad aumente y se reduzca su contenido en oxígeno, permitiéndole ser una masa de agua enriquecida en nutrientes y con bajos valores de $\delta^{13} \mathrm{C}$ (Talley, 1993). La NPIW sido encontrada en el océano Pacífico ecuatorial a profundidades de entre $~ 500$ a 1000 m (Mix et al., 2003).

La masa de Agua Intermedia Antartica ("Antarctic Intermediate Water", AAIW), también está caracterizada por un mínimo de salinidad (34.0-34.4 psu) que la sitúa en el rango de la NPIW $\left(27.3 \sigma_{\theta}\right)$, pero llega a ser más densa que la NPIW, fluyendo bajo los $900 \mathrm{~m}$ de profundidad (Talley, 1985; Tsuchiya y Talley, 1996). La AAIW es la masa de agua intermedia de máxima concentración de oxígeno en el Pacífico oriental (240-280 $\mu \mathrm{mol} / \mathrm{Kg})$, lo cual hace referencia a su último contacto con la atmósfera en la región de formación; por el contrario, la NPIW se caracteriza por una lengua de mínimo oxígeno $(130 \mu \mathrm{mol} / \mathrm{Kg})$ y una fuerte estratificación, indicando un origen diferente para ambas masas de agua (You, 2005). Adicionalmente, a diferencia de la AAIW que aflora en su región de formación cerca de la Antártida, la densidad de la NPIW no ha sido observada en superficie durante el invierno en todo el Pacífico Norte; esto indica que la NPIW no se forma por convección en el océano abierto durante el invierno, sino por procesos interiores lentos (You, 2005). El contacto con la atmósfera de la AAIW hace que esta masa de agua se empobrezca en fosfato y se enriquezca en oxígeno, registrando valores relativamente altos de $\delta^{13} \mathrm{C}$ (Kroopnick, 1985) (Fig. 3.4).

El Agua Pacífica Central-PCW ("Pacific Central Water") también se origina en el océano Pacífico norte, está asociada a mayores temperaturas y salinidad, y define la zona de mínimo oxígeno más profunda en la cuenca ( 1500 a 2000 m). Las masas de agua NPIW y PCW reflejan así la presencia de dos zonas de mínimo oxígeno-OMZ (“Oxygen Mynimum Zone”) relacionadas con su escasa interacción con la atmósfera en sus lugares de origen, así como al altísimo flujo de materia orgánica en los sistemas de surgencia del océano Pacífico nororiental (Tsuchiya y Talley, 1998). Estas masas de agua de mínimo oxígeno en el Pacífico oriental tropical junto con aquellas del mar de Arabia, son los principales lugares de denitrificación, y definen la mayor tasa de descenso de nitrógeno respecto a todos los océanos del mundo, adicionalmente actúan como reóstatos climáticos porque alteran la fertilidad y la tasa de fijación del $\mathrm{CO}_{2}$ en el océano (Mix et al., 2003). 
Adicionalmente a las masas de agua intermedia, las masas de agua profunda y de fondo del Pacífico oriental bañan la región más profunda de la cuenca (>3000 m) y entran a la cuenca de Panamá a través de valles en la Dorsal de Carniege en el ecuador (Lonsdale, 1976; Tsuchiya y Talley, 1998). El Agua Profunda Circumpolar-CPDW ("Circumpolar Deep Water", Fig. 3.4) se caracteriza por estar levemente enriquecida en oxígeno y empobrecida en nutrientes; sin embargo, en su recorrido hacia el norte, acumula nutrientes y pierde oxígeno (Mix et al., 2003). Las aguas de fondo ("Pacific Botton Water", PBW) están separadas de las aguas profundas que las suprayacen, por un frente bentónico (también conocido como termoclina bentónica) de aproximadamente $100 \mathrm{~m}$ de espesor, con un gradiente de temperatura de $0.3^{\circ} \mathrm{C} / 100 \mathrm{~m}$, y una medida promedio de velocidad de la corriente que varía de $16.5 \mathrm{~cm} / \mathrm{sec}$ a $21 \mathrm{~cm} / \mathrm{sec}$; lo que indica que la tasa de llegada del agua de fondo a la cuenca de Panamá, reemplaza el agua anterior en esa cuenca en un tiempo menor a los 50 años (Lonsdale, 1977).

\subsection{Océano Atlántico occidental tropical: cuenca de la Guayana}

\subsubsection{Escenario tectónico}

La cuenca de la Guayana se encuentra localizada al noreste de la placa Suramericana, en la Dorsal de Demerara. Esta placa se extiende desde Suramérica continental hasta la Dorsal Mesoatlántica ("Mid-Atlantic Ridge"), por ello su movimiento se ha dado a partir de la Dorsal Mesoatlántica una vez se produjo la rotura de Gondwana. El movimiento de la placa Suramericana le ha significado a la misma placa un proceso de compresión/acortamiento (a una tasa actual de $1 \mathrm{~cm} /$ año) debido principalmente a la convergencia entre las placas Suramericana y Nazca, y a la divergencia entre las placas Suramericana y Africana. Esta tasa de acortamiento ha permitido pronosticar que la magnitud de la compresión en los Andes debido al movimiento de la placa ha sido próxima a los 120 Km (Silver, 1998) (Fig. 3.1).

Aunque la geometría de los márgenes occidental y oriental de la placa Suramericana está bien definida, la geometría y la naturaleza tectónica de su margen sur y particularmente de su margen norte son muy complejas (Meijer y Wortel, 1992). El margen occidental de la placa Suramericana corresponde a un margen convergente en el cual subducen las placas Nazca y Antártica, dando lugar al levantamiento de la cordillera de los Andes y a la formación de la fosa Chileno-Peruana. Sin embargo, la tasa de subducción de la placa Antártica ocurre a una tasa inferior a la de la 
subducción de la placa Nazca, permitiendo que el segmento más austral de Chile sea caracterizado por un bajo nivel de actividad sísmica comparado con el resto del margen occidental de la placa Suramericana (Meijer y Wortel, 1992). La placa Antártica no sólo forma parte del límite occidental de la placa Suramericana, sino que junto con la placa Escocesa también define el límite sur de la placa Suramericana. El margen oriental de la placa Suramericana es un margen divergente respecto a la placa Africana permitiendo la formación del océano Atlántico y la Dorsal Transatlántica. El margen norte de la placa Suramericana limita con las placas Norteamericana y Caribe, pero la geometría y la naturaleza de este margen norte son complejas debido a que no es posible diferenciar bien el límite con la placa Norteamericana y además porque el límite norte de la placa Suramericana intersecta el arco de Las Antillas Menores (al oeste del arco de Las Antillas). El límite de las placas Suramericana y Caribe se caracteriza por una zona de deformación compleja el cual refleja que el componente este-oeste del movimiento relativo de estas placas predomina sobre el componente norte-sur. La subducción de la placa Caribe bajo la placa Suramericana se ha comprobado a través de la deformación de los sedimentos más recientes en el cinturón de deformación del sur de la placa Caribe. No obstante, esta zona de subducción no se caracteriza como una zona de alta actividad sísmica superficial como lo es la zona norte de los Andes (Meijer y Wortel, 1992). La velocidad aproximada del movimiento de la placa Suramerica respecto a la placa Caribe fue calculado en unos $2.3 \pm 0.5$ cm/año en dirección N75W $\left( \pm 10^{\circ}\right)$ (Bosch y Rodríguez, 1992).

Los sedimentos del testigo MD03-2616 extraídos de la cuenca de la Guayana (Shipboard Scientific Party, 2003) sobre la Dorsal de Demerara (Fig. 3.5). La Dorsal de Demerara es una plataforma submarina prominente localizada entre las costas de Surinam y la Guayana Francesa, la cual se extiende $\sim 380 \mathrm{Km}$ a lo largo de la costa y $\sim 220 \mathrm{Km}$ a lo ancho desde el final de la plataforma continental hasta el escarpe noreste donde la profundidad del agua se incrementa desde 1000 hasta mas de $4500 \mathrm{~m}$. La plataforma se encuentra a $700 \mathrm{~m}$ de profundidad, pero el margen noroeste corresponde a una rampa suave que alcanza profundidades de 3000 a $4000 \mathrm{~m}$ (Erbacher et al., 2004). La subsidencia de la cuenca y los cambios en el nivel del mar permitieron la acomodación de una gruesa cobertera sedimentaria de alrededor 2-3 Km de espesor (Mosher et al., 2007), la cual se adelgaza cerca del escarpe noreste, exponiendo la estratigrafía de la columna sedimentaria que yace sobre el basamento, el cual tiene una edad que va desde el Paleozoico hasta el Mesozoico temprano. 


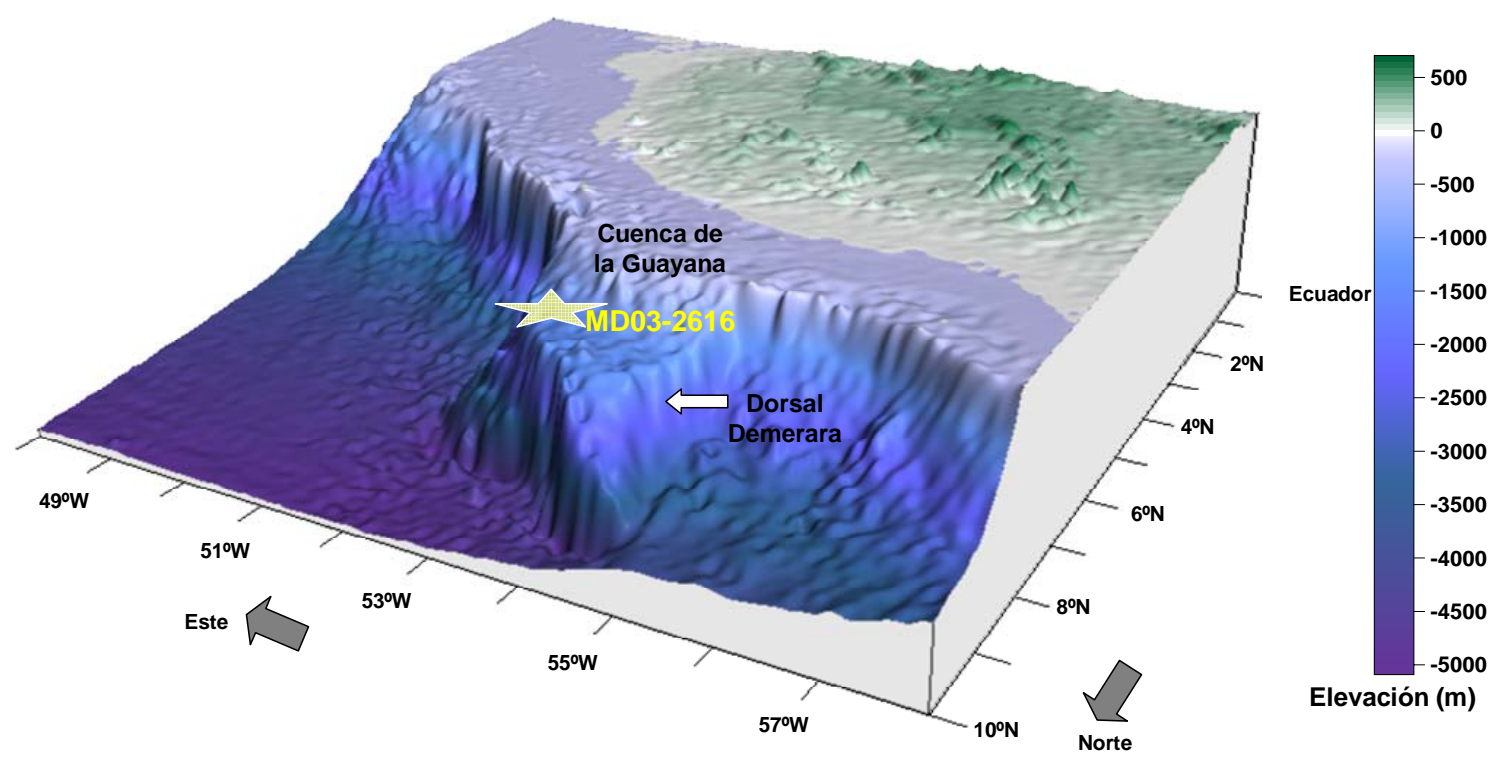

Fig. 3.5. Localización 3D del testigo MD03-2616 en la cuenca de la Guayana (Atlántico occidental tropical) y las variaciones del relieve en la cuenca. La escala de colores resalta las principales características batimétricas. Tomada de la base de datos topográfica de Smith y Sandwell, 1997.

\subsubsection{Oceanografía}

\section{Dinámica superficial y subsuperficial}

Las corrientes superficiales en el océano Atlántico occidental forman un giro anticiclónico subtropical bajo la influencia de los vientos alisios (Fig. 3.3). Las aguas superficiales en la franja tropical del océano Atlántico occidental son un poco más frías al sur que al norte del ecuador, en donde sobrepasan los $24^{\circ} \mathrm{C}$.

La región está influenciada principalmente por la corriente Surecuatorial ("South Equatorial Current", SEC) que circula en dirección este-oeste. Esta corriente, al llegar a Suramérica, se bifurca en la corriente del Brasil ("Brazil Current", BC), que fluye bordeando el continente en dirección sur, y la corriente Norte del Brasil ("North Brazil Current", NBC) también llamada corriente de la Guayana ("Guayana Current", GC) que bordea la costa en dirección norte. La interacción entre las corrientes NBC y SEC da paso al hundimiento de aguas superficiales en el Atlántico tropical de las cuales se alimentan la corriente Subsuperficial Ecuatorial ("Equatorial Undercurrent", EUC). Esta última corriente modifica su rango de profundidad entre 125 y $50 \mathrm{~m}$ a lo largo del ecuador hasta su afloramiento en la costa africana (Peterson y Stramma, 1991) (Fig. 3.3). 
La NBC se divide en dos brazos antes de llegar a la cuenca Cariaco, uno de ellos gira en dirección oeste-este alimentando la Contracorriente Norecuatorial ("North Equatorial Countercurrent"-NECC); y el otro brazo continúa en dirección norte, alimentando la corriente del Golfo ("Gulf Stream") y el Flujo Noratlántico ("North Atlantic Drift"), transfiriendo agua cálida y salina desde el ecuador hacia las altas latitudes del Atlántico Norte. El enfriamiento y el descenso de esta agua superficial en el océano Atlántico Norte forman la masa de Agua Profunda Noratlántica-NADW ("North Atlantic Deep Water") (Schmitz Jr. y McCartney, 1993). Tal dinámica superficial juega un papel muy importante en la circulación termohalina, como la principal componente del flujo de retorno de la NADW en la celda termohalina (Bassinot et al., 1997).

Durante el verano boreal la NBC se desvía hacia el océano abierto en torno a los 5 N-7ㅇN, para alimentar la NECC entre junio y diciembre (Nittrouer y DeMaster, 1986; Philander y Pacanowski, 1986; Richardson y Walsh, 1986), cuando la ITCZ está en su posición más norte. Durante el invierno boreal, la ITCZ está en su posición más sur, la NECC se debilita o tiende a desaparecer y la NBC sigue hacia el Noratlántico.

La estacionalidad de los vientos alisios está íntimamente relacionada con las fluctuaciones de la ITCZ y de las corrientes superficiales, lo cual se expresa en las variaciones de la profundidad de la nutri-termoclina. La nutri-termoclina en el océano Atlántico occidental tropical es relativamente más profunda que en el océano Atlántico oriental tropical a lo largo del año, pero durante el verano boreal la nutri-termoclina maximiza su profundidad en el Atlántico Occidental Tropical debido a la intensificación de las corrientes superficiales que acumulan una gran cantidad de masas de agua cálidas hacia el sector occidental.

La cuenca de la Guayana también recoge el agua de dos importantes cuencas de drenaje, las de los ríos Orinoco y Amazonas (Fig. 3.3), los dos ríos más caudalosos de Suramérica, que juntos suman el $19.5 \%$ del total del agua lluvia de la Tierra (Degens et al., 1991). Estos ríos junto con otros ríos de importancia relativamente menor, que drenan el área de las cuencas, depositan sus sedimentos en el océano Atlántico. El río Orinoco es el tercero más caudaloso del mundo (1.1×1012 m3/año, despues del Amazonas y el Zaire), pero ocupa el décimo primer puesto en descarga de sedimentos (3.0x1011 Kg/año; Milliman \& Meade, 1983). El río Amazonas es el más caudaloso del mundo (Milliman \& Meade, 1983) y su descarga media anual entre la plataforma de la Guayana y el ecuador es $(1.93 \pm 0.13) \times 10^{5} \mathrm{~m}^{3 *} \mathrm{~s}^{-1}$ (Perry et al., 1996). El río Orinoco drena 
directamente sobre el testigo MD03-2616 (Shipboard Scientific Party, 2003), pero el Amazonas realiza el mayor aporte de sedimentos al delta del Orinoco. Las principales fuentes de partículas transportadas por el río Amazonas se derivan de la erosión y meteorización física de detritos provenientes de los escudos de la Guayana y de Brasil, la Cuenca del Amazonas y especialmente de los Andes (Gibbs, 1967; Milliiman y Meade, 1983).

Los contrastes de temperatura y salinidad en el Atlántico occidental superficial son causados por la intervención del río Amazonas, dando lugar a anomalías de la temperatura del agua superficial $(28-29.7 \circ \mathrm{C})$ y de salinidad debido a la incorporación de agua dulce (Ffield, 2005). Por ello, las aguas que arrastra la NBC son más frías y saladas (35.9-36.2 \%) que las aguas circundantes.

Actualmente el ecuador térmico y la ITCZ se sitúan al norte del ecuador geográfico en el océano Atlántico occidental. Sus paleofluctuaciones han ejercido un papel importante en la circulación termohalina y en los casquetes del Atlántico Norte, debido a la modificación de la salinidad y el vapor de agua transportado desde el ecuador hacia las altas latitudes (Bassinot et al., 1997).

\section{Dinámica intermedia y profunda}

El océano Atlántico occidental también forma parte de la ruta principal de las masas de agua intermedias y profundas entre las altas latitudes del norte y del sur del Atlántico (Gröeger et al., 2003). La posición en profundidad del testigo MD03-2616 le convierte en un testigo potencial para intersectar las masas de agua intermedias en ese océano. Estas masas están constituidas por la capa superior de la masa de Agua Profunda Noratlántica ("Upper North Atlantic Deep Water", UNADW) y la masa de agua Antártica Intermedia ("Antarctic Intermediate Water", AAIW; Fig. 3.6).

La AAIW fluye a profundidades entre 800 y $1200 \mathrm{~m}$ y se extiende desde la región subantártica hacia el hemisferio norte en los océanos Atlántico, Pacífico e Índico (Suga y Talley, 1995). La AAIW se deriva del Agua Intermedia tipo Antártico (Antarctic Intermediate-ANI), la cual se forma en el Océano del Sur, en la zona de convergencia Antártica, lugar en el que se caracteriza por su relativa baja salinidad y alto contenido de oxígeno disuelto comparada con otras masas de agua atlánticas tropicales (Bub y Brown, 1996), además de su alto contenido en nutrientes, bajos valores de $\delta^{13} C$, baja densidad y temperaturas relativamente altas (Suga y Talley, 1995). Wüst (1935) trazó el paso de la AAIW en las aguas intermedias del océano Atlántico basándose en su propiedad más característica, que es el mínimo de salinidad. Ese mínimo de salinidad ha 
sugerido dos posibles trayectorias del flujo de la AAIW. Wüst (1935) indicó que la AAIW sigue un flujo continuo hacia el norte a lo largo del sistema occidental de circulación oceánica ("western boundary current"), permitiendo que la mayor parte de su flujo se dirija hacia el oriente antes de llegar al ecuador, y el resto del flujo siga la dirección hacia el norte. Otros autores (Taft, 1963; Buscaglia, 1971; Piola y Georgi, 1982; Warner y Weiss, 1992; Bub y Brown, 1996) han propuesto que la AAIW fluye hacia el norte hasta los 40S, luego gira en dirección este, luego hacia el norte y retorna al occidente bajo la SEC, siguiendo el giro anticiclónico subtropical para incorporarse de nuevo al sistema occidental de circulación oceánica ("western boundary current") en dirección norte bajo la NBC. Durante este viaje sus propiedades físicas van cambiando en el Hemisferio Sur a medida que se mezcla con otras masas de agua. Estas propiedades son modificadas a través del giro ciclónico subecuatorial, cerca de África, alrededor de los $10^{\circ} \mathrm{S}$, debido a que su paso por la célula de surgencia, hace que la AAIW experimente una pérdida brusca de oxígeno, un incremento fuerte de nitrato y fosfato y un incremento relativo de su salinidad (Reid, 1989; Bub y Brown, 1996). En general, las masas de agua intermedias del Atlántico Norte subtropical se diferencian de las masas de agua intermedias del Atlántico Sur subtropical en que las primeras son relativamente más salinas, más cálidas y contienen más oxígeno disuelto (Bub y Brown, 1996). LA AAIW se ha identificado a latitudes que llegan incluso a los $60 \mathrm{~N}$, siguiendo la ruta de la corriente del Golfo, siendo caracterizada en ese lugar por el máximo de sílice (Tsuchiya, 1989), aunque su particular mínimo de salinidad al norte de los $20{ }^{\circ} \mathrm{N}$ es poco clara (Suga y Talley, 1995).

Las masas de agua AAIW y UNADW están separadas por un máximo de salinidad el cual está asociado a la influencia de la masa de Agua Intermedia Mediterránea (MIW) sobre UNADW (Bub y Brown, 1996). La circulación de la AAIW en el océano Atlántico es particularmente interesante debido a que su dirección hacia el Atlántico Norte, le permite formar parte de la célula de circulación meridional asociada a la formación de la NADW (Rintoul, 1991; Gordon et al., 1992; Fine, 1993) siendo una de las principales fuentes de calor de la NADW (Suga y Talley, 1995). La NADW fluye hacia el sur entre $\sim 1200$ a $\sim 3900$ m (Rhein et al., 1996) y se caracteriza porque es una masa de agua profunda muy oxigenada, empobrecida en nutrientes y supersaturada en carbonato. Está dividida en dos capas, la masa de Agua Profunda Noratlántica Inferior ("Lower North Atlantic Deep Water", LNADW) originada en el Mar de Noruega en el Atlántico Norte, el cual es su fuente principal cuando el Frente Polar está sobre la costa de Groenlandia (Duplessy et al., 1988). La UNADW fluye bajo la AAIW está caracterizada por su bajo contenido en nutrientes y valores de $\delta^{13} \mathrm{C}$, de salinidad y de densidad relativamente altos (Bickert et al., 2004), 

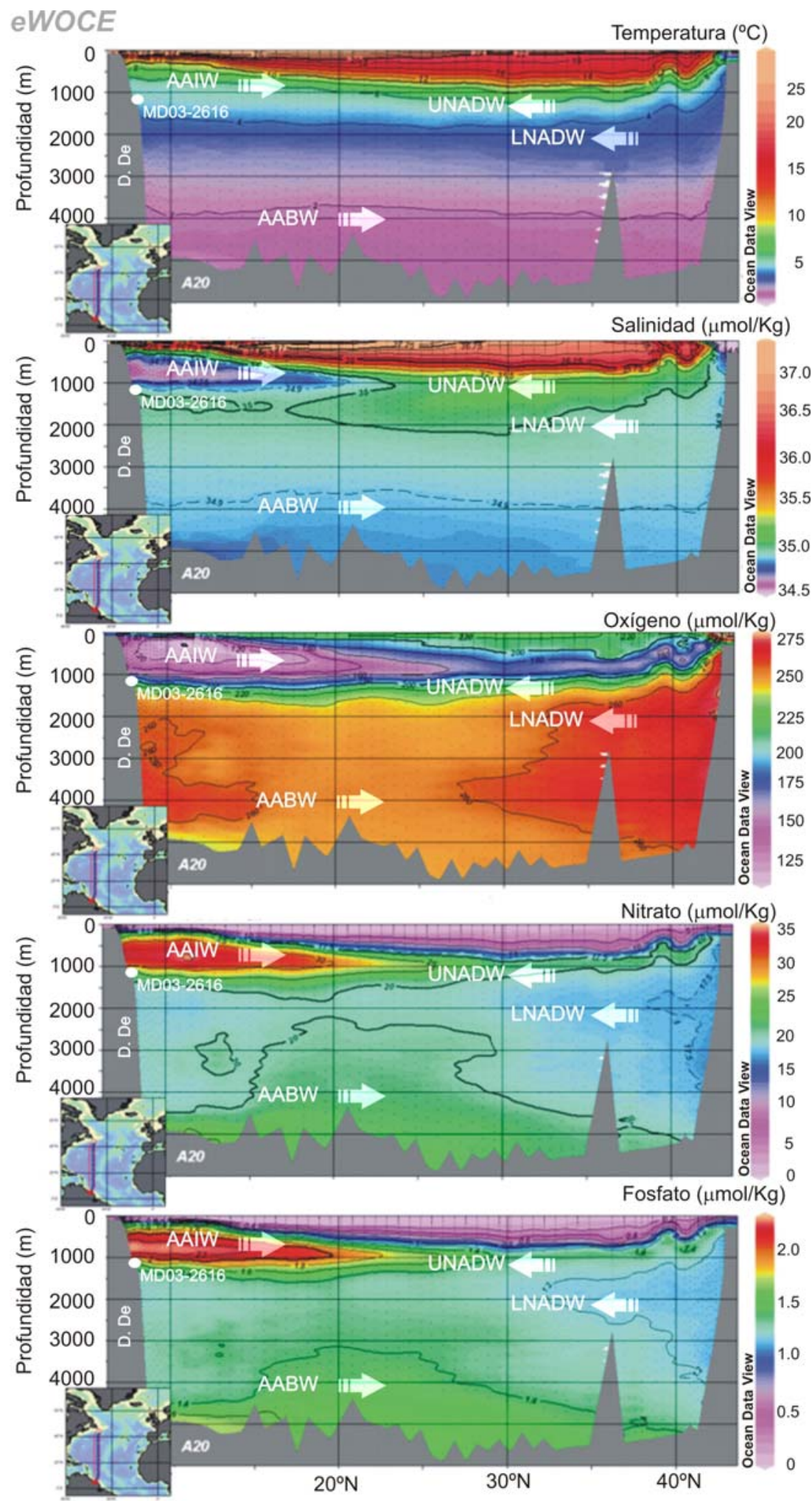

Fig. 3.6. Configuración de las masas de agua intermedias y profundas en el océano Atlántico occidental tropical y algunas de sus propiedades fisico-químicas (temperatura, salinidad, oxígeno, nitrato y fosfato), en el transecto paleoceanográfico meridional A20 tomada del eWOCE (World Ocean Circulation Experiment) (Schlitzer, 2000). AAIW, Agua Antarctica Intermedia; U/L NADW, Agua Profunda Noratlántica Superior/Inferior; AABW, Agua Antartica de Fondo; D.De, Dorsal de Demerara. 
y es una mezcla entre el Agua del Mar del Labrador (Labrador Sea Water", LSW) y la MIW (Oppo y Fairbanks, 1987).

Las masas de agua más profundas están ocupadas por la masa de Agua Antártica de Fondo (Antarctic Bottom Water-AABW). La zona de mezcla entre la NADW y la AABW está marcada por fuertes gradientes en el contenido de nutrientes, $\delta^{13} C_{D I C}$, DIC (Kroopnick, 1985).

Los fondos marinos del Atlántico Occidental tropical juegan además otro papel importante en el registro de las migraciones verticales entre las masas de agua profundas nor- y suratlánticas. A profundidades mayores de $3750 \mathrm{~m}$, la Dorsal Mesoatlántica obstaculiza la entrada de las aguas profundas y corrosivas del océano Antártico desde el Atlántico ecuatorial occidental hacia el oriental, permitiendo la profundización de la lisoclina al oriente.

\subsection{Los Andes: Geología y clima}

El levantamiento de la cordillera de los Andes por encima de los $4000 \mathrm{~m}$ de altitud y los cambios en su configuración, han ocasionado cambios significativos en el clima de Suramérica, la reorganización de la circulación atmosférica, la circulación oceánica manejada por los vientos alisios, la productividad superficial del océano, en el ciclo hidrológico (Philander et al., 1996; Xie y Saito, 2001; Xu et al., 2004) y en el desarrollo paleobiogeográfico del norte de Suramérica (Hoorn, et al., 1995).

El sistema Andino descansa en el límite de subducción entre las placas Nazca y Suramérica, y se extiende por más de $50^{\circ}$ de latitud (Cahill e Isacks, 1992) (Figs. 3.1, 3.3). El levantamiento de los Andes durante el Neógeno-Cuaternario evolucionó a partir de la reorganización en la tectónica de placas. La placa Farallón se rompió y esta división dio origen a las placas Nazca y Cocos al final del Oligoceno tardío (Fig. 3.1), y a un centro de expansión que aumentó la tasa de convergencia en las márgenes continentales (Wortel y Cloetingh, 1981; Wortel, 1984). Tanto, el incremento en la tasa de covergencia ya observado durante una fase temprana de convergencia rápida en el Eoceno medio (Pardo-Casas y Molnar, 1987), así como el desplazamiento oesteeste de la placa Caribe que hizo que el Istmo de Panamá se cerrara entre los 3.7-3.4 Ma, activaron la actividad tectónica en los Andes (Sykes et al., 1982; Duque-Caro, 1990). 
Todos estos eventos tectónicos permitieron igualmente una reorganización en los patrones de drenaje en el norte de Suramérica durante el Mioceno (Hoorn, et al., 1995), influyendo en el desarrollo de los ríos Orinoco y Amazonas (Johnsson et al, 1991). El Mioceno medio fue marcado por el comienzo del levantamiento de los Andes nororientales y representa el episodio más dinámico de su levantamiento en todo el Mioceno (Keller y Barrol, 1987; Hoorn et al., 1995), lo cual coincidió con un tectonismo global, asociado a un evento de enfriamiento importante, como fue el establecimiento del casquete de hielo de la Antártida (Hoorn, et al., 1995) y a un hiato en el océano profundo entre los 12.9-11.8 Ma (Keller y Barrol, 1987). El río Amazonas pasó a realizar un drenaje transcontinental y cubrió las plataformas carbonatadas del Atlántico. Al mismo tiempo, el Orinoco cambió su curso y abandonó el Lago Maracaibo. La conexión Amazonas-Caribe se cerró por los eventos tectónicos del Mioceno tardío y por un descenso relativo del mar a nivel global (Hoorn, et al., 1995). El levantamiento máximo de los Andes durante el Plioceno (5 Ma) es coincidente con la subducción de la Dorsal de Cocos, formada durante el paso de la placa de Cocos sobre el punto caliente de Galápagos, y la elevación del arco volcánico de Centroamérica permitiendo el cierre del Istmo de Panamá (Dengo, 1985). Las etapas de máximo levantamiento y los cambios regionales del clima han quedado grabados en los sedimentos suministrados por el Amazonas hacia el océano Altántico occidental (Clayton et al., 1999). Durante el Mioceno tardío y el Holoceno, los Andes adquirieron su actual configuración (Hoorn, et al., 1995).

Los Andes actúan como barrera para los vientos alisios en el subtrópico y para los vientos que vienen del oeste ("Westerlies") en las regiones de latitudes altas y medias de Suramérica. Actualmente la interrupción del paso de los "Westerlies" en esas latitudes, causa precipitación en los glaciares de Chile y la Patagonia (al oeste de la cordillera), mientras que causa aridez en el desierto de la Patagonia (al este de la cordillera). Pero este patrón se invierte a bajas latitudes: Al oriente de los Andes, los vientos alisios canalizan el aire marítimo tropical en dirección suroriental y causan fuertes precipitaciones influyendo en el clima de la Amazonía, permitiendo el desarrollo de los ríos Amazonas y Orinoco entre otros importantes ríos, mientras que hacia el occidente de la cordillera, los vientos alisios causan sequedad (desierto de Atacama). Los Andes restringen el límite oriental de la célula de alta presión y hacen que los vientos sigan la línea de costa, dando lugar a la intensificación de la surgencia costera, las bajas temperaturas del océano superficial, la escasa evaporación y a la intensificación de la aridez a lo largo de la costa occidental de Suramérica (Hay, 1996; Mix et al., 2003). 
La topografía de los Andes hace que la posición de la ITCZ al occidente de la cordillera se encuentre siempre en el Hemisferio Norte, favoreciendo la presencia de temperaturas más altas en la franja tropical norte que en la del sur. Si los Andes fueran eliminados, el aire cálido continental soplaría directamente sobre el océano Pacífico oriental, reduciría la divergencia en el ecuador y favorecería la convergencia al sur del ecuador. Durante la estación más cálida, los efectos barrera de los Andes influyen sobre la precipitación en Suramérica y sobre el desplazamiento latitudinal de la ITCZ siempre al norte del ecuador en el océano Pacífico oriental. Durante la estación más fría en el hemisferio austral, los Andes bloquean los alisios que soplan desde el continente favoreciendo la divergencia oceánica que impide la presencia de temperaturas superficiales cálidas en el océano al sur del ecuador (Xu et al., 2004).

Los Andes además, son la principal fuente de detritos transportados por el río Amazonas. Actualmente, las cuencas de drenaje de los ríos Amazonas y Orinoco, están caracterizadas por un ambiente cálido y húmedo asociadas a la selva tropical, lo cual implica altas tasas de meteorización química y suelos ricos en cuarzo y en aluminio (McDaniel et al., 1997). Durante las glaciaciones del Pleistoceno, el volumen de las nieves perpetuas de los Andes se incrementó, favoreciendo un ambiente terrestre más árido que el actual en Suramérica (McDaniel et al., 1997), pero durante los epidodios de deglaciación, el derretimiento de los hielos de los Andes pudieron haber causado un aumento en la descarga de agua dulce, detritos (Maslin y Mikkelsen, 1997), materia orgánica al Atlántico occidental, y valores más bajos en la señal del $\delta^{180}$ en el océano (Maslin et al., 1997).

\section{Referencias}

Barckhausen, U., Ranero, C., von Huene, R., Cande, S. S., Roeser, H. A. 2001. Revised tectonic boundaries in the Cocos Plate off Costa Rica: Implications for the segmentation of the convergent margin and for plate tectonic models. Journal of Geophysical Research 106(B9),19207-19220.

Bassinot, F. C., Beaufort, L., Vincent, E., and Labeyrie, L. 1997. Changes in the dynamics of Western Equatorial Atlantic surface currents and biogenic productivity at the "Mid-Pleistocene Revolution" ( $930 \mathrm{Ka})$, in: Proc. ODP, edited by: Shackleton, N. J., Curry, W. B., Richter, C., and Bralower, T. J., Sci. Results, 154: College Station, TX (Ocean Drilling Program), 269-284.

Bickert, T., Haug, G.H., Tiedemann, R. 2004. Late Neogene benthic stable isotope record of Ocean Drilling Program Site 999: Implications for Caribbean paleoceanography, organic carbon burial, and the Messinian Salinity Crisis. Paleoceanography 19, doi:10.1029/2002PA000799.

Bosch, M., Rodríguez, I. 1992. North Venezuelan collisional crustal block: The boundary between the Caribbean and South American plates. Journal of South American Earth Sciences 6, 133 - 143.

Bub, F. L., Brown, W.S. 1996. Intermediate layer water masses in the western tropical Atlantic Ocean. Journal of Geophysical Research 101 (C5), 11,903-11,922.

Buscaglia, J.L. 1971. On the circulation of the intermediate water in the southwestern Atlantic Ocean. Journal of Marine Research 29, 245-255. 
Cahill, T., Isacks, B. 1992. Seismicity and shape of the subducted Nazca plate. Journal of Geophysical Research, 17503-17529

Canales, J. P., Danobeita, J. J., Detrick, R. S., Hooft, E. E. E., Bartolome, R., Naar, D. F. 1997. Variations in axial morphology along the Galapagos spreading center and the influence of the Galapagos hotspot. Journal of Geophysical Research 102(B12), 27341-27354

Clayton, T., Pearce, R.B., Peterson, L.C. 1999. Indirect climatic control of the clay mineral composition of Quaternary sediments from the Cariaco basin, northern Venezuela (ODP Site 1002). Marine Geology 161, 191-206.

Clement, A.C., Seager, R., Cane, M.A. 1999. Orbital controls on the El Niño/Southern Oscillation and the tropical climate. Paleoceanography 14, 441-456.

Degens, E.T., Kempe, S., Richey, J.E. 1991. Summary: biogeochemistry of major world rivers. In: Degens, E.T., Kempe, S., Richey, J.E. (Eds.), Biogeochemistry of Major World Rivers. Wiley, New York, pp. 323-347.

Dengo, G., 1985. Tectonic setting for the Pacific margin from southern Mexico to northwestern Columbia. In Naim, A.E., Stehli, F.G., and Uyeda, S. (Eds.), The Pacific Ocean: The Ocean Basins and Margins, 7A: New York (Plenum Press), 123-180.

Duplessy, J.-C., Shackleton, N.J., Fairbanks, R.G., Labeyrie, L., Oppo, D., Kallel, N. 1988. Deepwater source variations during the last climatic cycle and their impact on the global deepwater circulation. Paleoceanography $3,343-360$.

Duque-Caro, H. 1990. Neogene stratigraphy, paleoceanography and paleobiogeography in northwest South America and the evolution of the Panama seaway. Palaeogeography, Palaeoclimatology, Palaeoecology, 77(3-4):203-234. doi:10.1016/0031- 0182(90)90178-A

Erbacher, J., Mosher, D.C., Malone, M.J., et al., 2004. Proc. ODP, Init. Repts., 207: College Station, TX (Ocean Drilling Program). doi:10.2973/odp.proc.ir.207.2004

Fedorov, A.V., Philander, G. 2000. Is El Niño changing? Science 288, 1197-2002.

Ffield, A. 2005. North Brazil current rings viewed by TRMM Microwave Imager SST and the influence of the Amazon Plume. Deep-Sea Research I 52, 137-160.

Fiedler, P.C., Talley, L.D. 2006. Hydrography of the eastern tropical Pacific: A review. Progress in Oceanography $69,143-180$.

Fine, R.A. 1993. Circulation of Antarctic Intermediate Water in the South Indian Ocean. Deep-Sea Research Part I 40, 2021-2042.

Gibbs, R.J. 1967. Geochemistry of the Amazon River system, 1. The factors that control the salinity and the composition and concentration of the suspended solids. Geological Society of America Bulletin 78, 1203-1232.

Gordon, A.L. Weiss, R.F., Smethie Jr., W.M., Warner, M.J. 1992. Thermocline and Intermediate Water communication between the South Atlantic and Indian Oceans. Journal of Geophysical Research 97, 7223-7240.

Gröeger, M., Henrich, R., Bickert, T. 2003. Variability of silt grain size and planktonic foraminiferal preservation in Plio/Pleistocene sediments from the western equatorial Atlantic and Caribbean. Marine Geology 201, 307-320.

Hauff, F., Hoernle, K., Schminke, H.-U., Werner, R., 1997. A mid-Cretaceous origin for the Galapagos hotspot: volcanological, petrological, and geochemical evidence from Costa Rican oceanic crustal segments. Geol. Rundsch. 86, 141-155. doi:10.1007/PL00009938

Haug, G.H., Günther, D., Peterson, L.C, Sigman, D.M., Hughen, K.A., Aeschlimann, B. 2003. Climate and the collapse of Maya Civilization. Science 299, 1731-1735.

Hay, W.W. 1996. Tectonics and climate. Geologische Rundschau 85, 409-437.

Hey, R. 1977. Tectonic evolution of the Cocos-Nazca spreading center. Bulletin Geological Society of America 88, 1404-1420

Hoernle, K., Werner, R., Morgan, J. P., Garbe-Sch"onberg, D.,Bryce, J., Mrazek, J. (2000). Existence of complex spatial zonation in the Galápagos plume for at least 14 m.y. Geology 28, 435-438.

Hofman, E., Busalacchi, A.J., Q'Brien J. 1981. Wind generation of the Costa Rica Dome. Science 214, 552-554. doi: 10.1126/science.214.4520.552.

Hoorn, C., Guerrero, J., Sarmiento, G.A., Lorente, M.A. 1995. Andeantectonics as a cause for changing drainage patterns in Miocene northern South America. Geology 23, 237-240. 
Johnsson, M.J., Stallard, R.F., Lundberg, N. 1991. Controls on the composition of fluvial sands from a tropical weathering environment; sands of the Orinoco River drainage basin, Venezuela and Colombia. Geological Society of America Bulletin 103, 1622-1647

Jousaumme, S., Sadourny, R., and Vignal, C. 1986. Origin of precipitating water in a numerical simulation of the July climate. Ocean-Air Interactions 1, 43-56.

Keller , G., Barron, J.A. 1987. Paelodepth distribution of Neogene deep-sea hiatuses. Paleoceanography 2, 697-713.

Kessler, W.S. 2006. The circulation of the eastern tropical Pacific: a review. Progress in Oceanography 69, 181-217.

Kroopnick, P.M. 1985. The distribution of $\delta^{13} \mathrm{C}$ of $\mathrm{CO}_{2}$ in the world oceans. Deep-Sea Research 32, 57-84.

Lonsdale, P. 1976. Abyssal circulation of the southeastern Pacific and some geological implications. Journal of Geophysical Research 81, 1163-1176.

Lonsdale, P. 1977. Inflow of bottom water to the Panama Basin. Deep-Sea Research 24, 1065-1101.

Lonsdale, P., Klitgord, K.D. 1978. Structure and tectonic history of the eastern Panama Basin. Geological Society America Bulletin 89, 981-999.

Magaña, V.O., Amador, J.A., Medina, S. 1999. The mid-summer drought over Mexico and Central America. Journal of Climate 12, 1577-1588.

Maslin, M., Mikkelsen, N. 1997. Amazon Fan mass transport deposits and underlying interglacial deposits: age estimates and fan dynamics: Leg 155. In: Flood, R.D., Piper, D.W.J., Klaus, A., Peterson, L.C. (Eds.). Proc. ODP, Sci. Results, 155: College Station, TX (Ocean Drilling Program), 353365.

Maslin, M., Burns, S., Erlenkeuser, H., Hohnemann, C. 1997. Stable isotope records from sites 932 and 933: Leg 155. In: Flood, R.D., Piper, D.W.J., Klaus, A., Peterson, L.C. (Eds.). Proc. ODP, Sci. Results, 155: College Station, TX (Ocean Drilling Program), 305-318.

McDaniel, D. K., McLennan, S.M., Hanson, G.N. 1997. Provenance of Amazon fan muds: constraints from $\mathrm{Nd}$ and $\mathrm{Pb}$ isotopes: Leg 155. In: Flood, R.D., Piper, D.W.J., Klaus, A., Peterson, L.C. (Eds.). Proc. ODP, Sci. Results, 155: College Station, TX (Ocean Drilling Program), 169-176.

Meijer, P.Th., Wortel, M.J.R. 1992. The dynamics of motion of the South American Plate. Journal of Geophysical Research 97 (B8), 11,915-11,931.

Meissner, R., Flilh, E. R., Stibane, E, Berg, E. 1976. Dynamics of the active plate boundary in southwest Colombia according to recent geophysical measurements. Tectonophysics $35,15-136$.

Milliman, J.D., Meade, R.H. 1983. Worldwide delivery of river sediment to the oceans. Journal of Geology $91,1-21$.

Mix, A.C., Tiedemann, R., Blum, P., et al., 2003. Proc. ODP, Init. Repts., 202. Ocean Drilling Program, College Station, TX. doi:10.2973/odp.proc.ir.202.2003.

Mosher, D.C., Erbacher, J., Malone, M.J. 2007. Proc. ODP, Sci. Results, 207: College Station, TX (Ocean Drilling Program). doi:10.2973/odp.proc.sr.207.2007.

Nittrouer, C. A., DeMaster, D. J., 1986. Sedimentary processes on tile Amazon continental shelf: past, present and future research. Continental Shelf Research 6, 5-30.

Oppo, D.W., Fairbanks, R.G. 1987. Variability in the deep and intermediate water circulation of the Atlantic Ocean during the past 25,000 years: Northern Hemisphere modulation of the Southern Ocean. Earth and Planetary Science Letters 86, 1-15.

Pardo-Casas, F., Molnar, P. 1987. Relative motion of the Nazca (Farallon) and South American plates since late Cretaceous time. Tectonics 6, 233-248.

Pak, H., Zaneveld, J.R. 1974. Equatorial Front in the Eastern Pacific Ocean. Journal of Physical Oceanography 4, 570-578.

Pennington, W. D. 1981. Subduction of the Eastern Panama Basin and Seismotectonics of Northwestern South America. Journal of Geophysical Research 86 (B11):10753-10770

Perry, G.D., Duffy, P.B., Miller, N.L. 1986. An extended data set of river discharges for validation of general circulation models. Journal of Geophysical Research 101 (D16), 21,339-21,349.

Peterson, R.G., Stramma, L. 1991. Upper-level circulation in the South Atlantic Ocean. Progress in Oceanography 26, 1-73.

Philander, G. 1995. El Niño and La Niña. In: Pirie, R.G. (Ed.), Oceanography: Contemporary Readings in Ocean Sciences, Oxford Univ. Press, Oxford, U.K., 72-87. 
Philander, S.G.H., Pacanowski, R.C. 1986. A model of the seasonal cycle in the tropical Atlantic Ocean. Journal Geophysical Research 91, 14192-14206.

Philander, S.G.H., Gu, D., Halpern, D., Lambert, G., Lau, N.-C., Li, T., Pacanowski, R.C. 1996. Why the ITCZ is mostly north of the Equator. Journal of Climate 9, 2958-2972.

Piola, A., Georgi, D.T. 1982. Circumpolar properties of Antarctic and Subantarctic Mode Water. Deep-Sea Research 29, 687-711.

Rahmstorf, S. 1995. Bifurcations of the Atlantic thermohaline circulation in response to changes in the hydrological cycle. Nature 378, 145-150.

Reid, J.L. 1989. On the total geostrophic circulation of the South Atlantic Ocean flow patterns, tracers and transports. Progress in Oceanography 23, 149-244.

Rhein, M., Schott, F., Fischer, J., Send, U., Stramma, L. 1996. The deep water regime in the Equatorial Atlantic. In: Wefer, G., Berger, W.H., Siedler, G., Webb, D. (Eds.), The South Atlantic: Present and past circulation, Springer-Verlag, Heidelberg, pp. 261-271.

Richardson, P.L., Walsh, D. 1986. Mapping climatological seasonal variations of surface currents in the tropical Atlantic using ship drifts, Journal of Geophysical Research, 91, 10537-10550.

Richardson, P.L., Hufford, G., Limeburner, R., Brown, W. 1994. North Brazil Current retroflection eddies. Journal of Geophysical Research 99, 5081-5093.

Rintoul, S.R. 1991. South Atlantic interbasin exchange. Journal of Geophysical Research 96, 2675-2692.

Schlitzer, R. 2000. Electronic Atlas of WOCE Hydrographic and Tracer Data now available. EOS Transactions, American Geophysical Union 81 (5), 45.

Schmitz Jr., W.J., McCartney, M.S. 1993. On the North Atlantic circulation. Reviews of Geophysics 31, 2949.

Shipboard Scientific Party. 2003. MD132-PICASSO. IMAGES XI. Cruise Report. 76p.

Silver, P.G., Russo, R. M., Lithgow-Bertelloni, C. 1998. Coupling of South America and African Plate motion and Plate Deformation. Science 279, 60-63.

Smith, W.H.F., Sandwell, D.T. 1997. Global sea floor topography from satellite altimetry and ship depth soundings. Science 277, 1956-1962.

Suga, T., Talley, L.D. 1995. Antarctic Intermediate Water circulation in the tropical and subtropical South Atlantic. Journal of Geophysical Research 100 (C7), 13,441-13,453.

Sykes, L., McCann, W.R., Kafka, A.L. 1982. Motion of Caribbean plate during last 7 million years and implications for earlier Cenozoic movements. Journal of Geophysical Research 87 (B13), 10,65610,676

Taft, B.A. 1963. Distribution of salinity and dissolved oxygen on surfaces of uniform potential specific volume in the South Atlantic, South Pacific and Indian Oceans. Journal of Marine Research 21, 129-146.

Talley, L. 1985. Ventilation of the Subtropical North Pacific: The shallow salinity maximum. Journal of Physical Oceanography. 15, 633-649.

Talley, L.D. 1993. Distribution and formation of North Pacific intermediate water. Journal of Physical Oceanography 23, 517-537.

Tiedemann, R., Mix, A. 2007. Leg 202 synthesis: southeast Pacific paleoceanography. In Tiedemann, R., Mix, A.C., Richter, C., and Ruddiman, W.F. (Eds.), Proc. ODP, Sci. Results, 202: College Station, TX (Ocean Drilling Program), 1-56. doi:10.2973/odp.proc.sr.202.201.2007

Tsuchiya, M. 1989. Circulation of the Antarctic Intermediate Water in the North Atlantic Ocean. Journal of Marine Research 47, 747-755.

Tsuchiya, M., Talley, L.D. 1996. Water-property distributions along an eastern Pacific hydrographic section at $135^{\circ} \mathrm{W}$. Journal of Marine Research 54, 541-564.

Tsuchiya, M., Talley, L.D. 1998. A Pacific hydrographic section at $88^{\circ} \mathrm{W}$ : Waterproperty distribution. Journal of Geophysical Research 103, 12899-12918.

Warne, A.G., Meade, R.H., White, W.A., Guevara, E.H., Gibeaut, J., Smyth R.C., Aslan, A., Tremblay, T. 2002. Regional controls on geomorphology, hydrology, and ecosystem integrity in the Orinoco Delta, Venezuela. Geomorphology 44, 273-307.

Warner, M.J., Weiss, R.F. 1992. Chlorofluoromethanes in South Atlantic Antarctic Intermediate Wate. Deep-Sea Research 39, 2053-2075. 
Werner, R., Hoernle, K., van den Bogaard, P., Ranero, C., von Huene, R. 1999. Drowned 14-m.y.-old Galápagos archipelago off the coast of Costa Rica: Implications for tectonic and evolutionary models. Geology 276, 499-502

Wilson, D. S., Hey, R. 1995. History of rift propagation and magnetization intensity for the Cocos-Nazca spreading center. Journal of Geophysical Research 100(B7), 10041-10056

Wortel, R., Cloetingh, S. 1981. On the origin of the Cocos-Nazca spreading center. Geology 9, 425-430.

Wortel, R. 1984. Spatial and temporal variations in the Andean subduction zone. Journal of the Geological Society London 141, 783-791.

Wüst, G. 1935. Schichtung und Zirkulation des Atlantischen Ozeans, Die Stratosphäre. Wissenschaftliche Ergebnisse der Deutschen Atlantischen Expedition auf dem Forschungsund Vermessungsschiff "Meteor" 1925-1927. 6, 180pp. English translation edited by W.J. Emery, The stratosphere of the Atlantic Ocean. Scientific Results of the German Atlantic Expedition of the Research Vessel 'Meteor' 1925-27. Amerind Publishing Co., 1978.

Wyrtki, K. 1981. An estimate of equatorial upwelling in the Pacific. Journal of Physical Oceanography 11, 1205-1214.

Xie, S.-P., Saito, K. 2001. Formation and variability of a northerly ITCZ in a hybrid coupled AGCM: continental forcing and oceanic atmospheric feedback. Journal of Climate 14, 1262-1276.

Xu, Haiming, Wang, Y., Xie, S.-P. 2004. Effects of the Andes on Eastern Pacific climate : a regional atmospheric model study. Journal of Climate 17, 589-602.

You, Y. 2003a. The pathway and circulation of North Pacific Intermediate Water. Geophysical Research Letters 30, 2291. doi:10.1029/2003GL018561.

You, Y. 2003b. Implications of cabbeling on the formation and transformation mechanism of North Pacific Intermediate Water. Journal of Geophysical Research 108 (C5), 3134. doi:10.1029/2001JC001285.

You, Y. 2005. Unveiling the mystery of North Pacific Intermediate Water formation. EOS Transactions, American Geophysical Union, 86 (7), 65-67.

Zaucker, F., Stocker, T.F., Broecker, W.S. 1994. Atmospheric freshwater fluxes and their effect on the global thermohaline circulation. Journal of Geophysical Research 99(C6), 12443- 12458. doi:10.1029/94JC00526. 


\section{Materiales y Métodos}

4.1. Introducción

4.2. Las campañas oceanográficas y los sistemas de extracción de testigos

4.2.1. Campaña ODP 202

4.2.2. Campaña PICASSO

4.3. Descripción de los testigos

4.3.1. Testigo ODP 1240

4.3.2. Testigo ODP 1241

4.3.3. Testigo ODP 1242

4.3.4. Testigo MD03-2616

4.4. Técnicas de preparación de las muestras

4.4.1. Preparación de "láminas" para el análisis de cocolitóforos

4.4.2. Preparación de muestras para el análisis de isótopos de oxígeno $\left(\delta^{18} \mathrm{O}\right)$ en foraminíferos

4.5. Técnicas de cuantificación

4.5.1. Cálculo de la abundancia absoluta y estimación del flujo de cocolitos y del carbonato aportado

4.5.2. Cálculo del índice N

4.5.3. Determinación del grado de preservación del carbonato

4.6. Cronología

Referencias 


\subsection{Introducc ción}

El material estudiado procede de sendas campañas oceanográficas llevadas a cabo por una tripulación cientifica internacional y multidisciplinaria. La campaña 202 ("Leg 202") fue realizada en el año 2002 por el buque científico norteamericano "JOIDES Resolution" ("JOIDES" - "Joint Oceanographic Institutions for Deep Earth Sampling"), dentro del proyecto internacional de extracción de sondeos ODP ("Ocean Drilling Program"; Mix et al., 2003). Y la Campaña PICASSO ("Paleoclimatologie, Isotopes, Chimie, Atlantique, Séries Sedimentaires Océaniques"), llevada a cabo por el buque francés "Marion Dufresne" dentro del programa internacional IMAGES ("Internacional Marine Past Global Changes Study") en el año 2003 (Shipboard Scientific Party, 2003) (Fig. 4.1).

\subsection{Las campañas oceanográficas y los sistemas de extrac ción de testigos}

\subsubsection{Campaña ODP 202}

La campaña ODP 202 realizó la extracción de más de $7 \mathrm{Km}$ de sedimento continuo en once testigos localizados a lo largo de un transecto paleoceanográfico en el océano Pacífico suroriental, entre los $\sim 40^{\circ} S$ hasta los $\sim 7.5^{\circ} \mathrm{N}$ (Fig. 4.1), los cuales alcanzan los últimos $32 \mathrm{Ma}$ (Oligoceno temprano). El objetivo científico fundamental de la campaña 202 fue evaluar los cambios climáticos y oceanográficos y su papel en los sistemas biogeoquímicos en el Pacífico suroriental a escala tectónica (millones de años), orbital (decenas a centenares de miles de años) y secular a milenaria (cientos de miles de años) a través de un conjunto de objetivos específicos basados en las escalas de tiempo mencionadas (Mix et al., 2003):

a. Explicar la respuesta del océano Pacífico Sur a los principales eventos tectónicos y climáticos, tales como la apertura del estrecho de Drake (que generó una corriente circumpolar), el levantamiento de la cordillera de los Andes (que modificó el sistema de vientos), el cierre del istmo de Panamá (que separó los océanos Atlántico y Pacífico) y la principal expansión de los casquetes de hielo polar a altas latitudes en los hemisferios norte y sur durante diferentes períodos. 
b. Estudiar la relación del cambio climático ocurrido en las altas latitudes del Hemisferio Sur y aquel en el Pacífico ecuatorial, asociados a los cambios rítmicos en la órbita de la Tierra, y la relación de esos cambios con los eventos glaciales/interglaciales bien descritos para el Hemisferio Norte.

c. Analizar los cambios globales y regionales del clima, la biota y la química del océano a escala secular-milenaria, los cuales aunque han sido detectados en diferentes sectores a nivel global, necesitan más dedicación, con la finalidad de analizar el vínculo existente entre estos sectores, e investigar los mecanismos que han originado esos cambios, bien sea a altas 0 a bajas latitudes.

Los testigos 1240, 1241 y 1242, localizados en la cuenca de Panamá entre el ecuador y $7.5^{\circ} \mathrm{N}$, fueron elegidos para evaluar la respuesta de los cocolitóforos a los cambios climáticos a escala glacial/interglacial en la zona de divergencia ecuatorial -conocida también como Lengua Fría Ecuatorial- y en la "warm pool" de la cuenca de Panamá, durante el Pleistoceno medio y tardío: (los últimos 600 Ka para el testigo ODP 1240, MIS 1 al 15; los últimos 830 Ka para el testigo ODP 1241, MIS 1 al 20; y los últimos $925 \mathrm{Ka}$ para el testigo ODP 1242, MIS 1 al 22). La ubicación geográfica de estos testigos está sujeta a las interacciones de los vientos alisios, la divergencia y las células de surgencia de aguas frías ricas en nutrientes que dan lugar a los niveles más altos de "nueva" producción biológica primaria vista en todo el océano a nivel global (Chávez y Barber, 1987; ver sección 2.6).

La estrategia seguida para la extracción de las secuencias sedimentarias en las campañas ODP tiene como objetivo principal obtener múltiples pozos para cada testigo, con el fin de recuperar secciones estratigráficas completas, de gran longitud y con la menor perturbación de extracción posible, a través de los sistemas ACP ("Advanced Hydraulic Piston Corer") y XCB ("Double Extended Core Barrel").

El sistema APC es la técnica preferida para la extracción de sedimentos de las campañas ODP porque la tubería presurizada corta el testigo sin deformar el sedimento. Cuando no se logra el objetivo en profundidad con la técnica APC - bien sea porque la formación a cortar es demasiado dura - se utiliza la técnica XCB para avanzar hacia el fondo. Esta última técnica se caracteriza por un sistema rotatorio que incorpora un "pie cortador", y que puede ocasionar perturbaciones en el sedimento. Una vez se extraen los pozos de cada testigo, los datos de 
profundidad de sus secuencias sedimentarias se catalogan en metros bajo el nivel del mar (“meters below sea floor", mbsf).

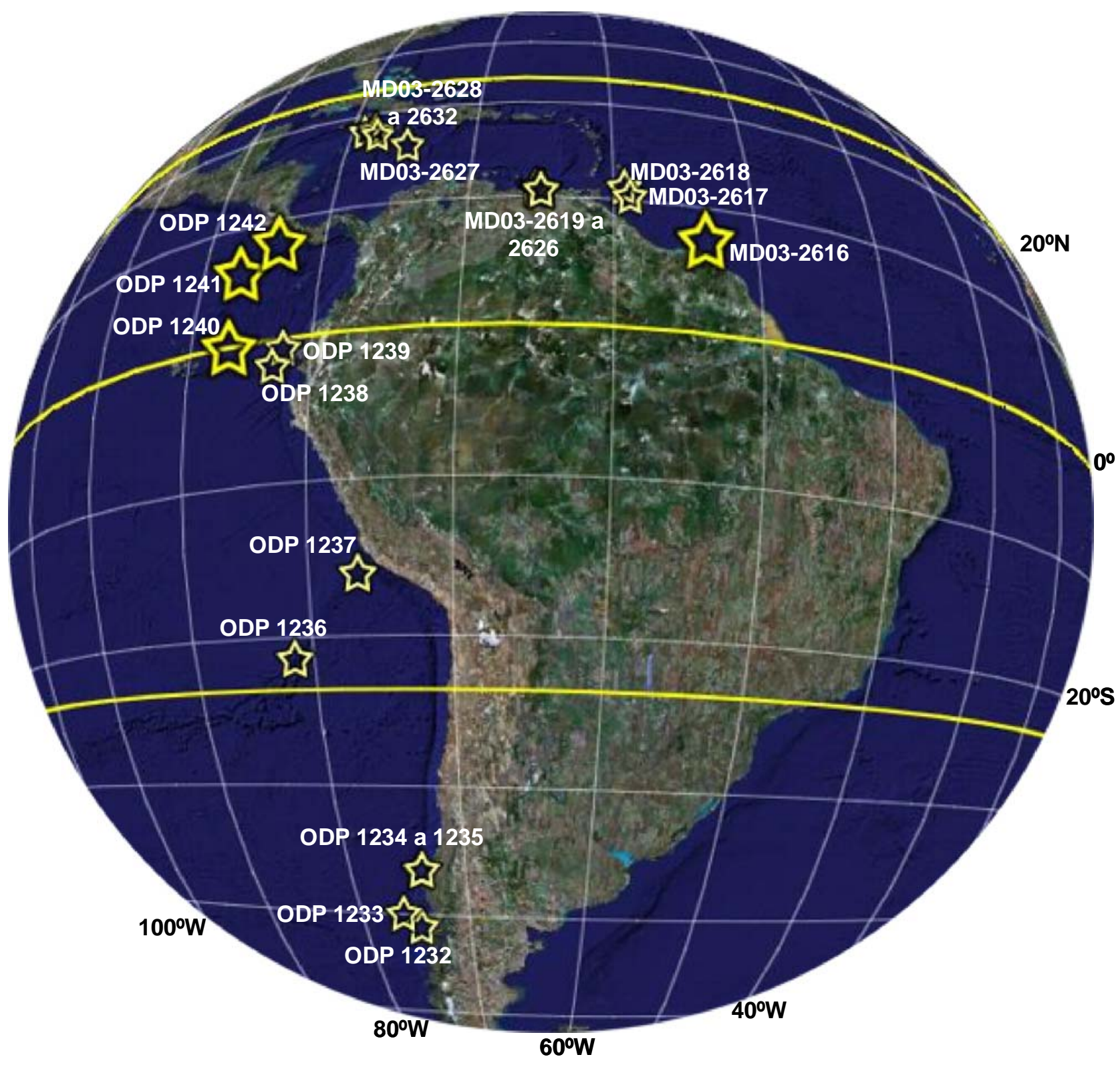

Fig. 4.1. Testigos extraídos por las campañas oceanográficas Ocean Drilling Program (ODP) 202 (Mix et al., 2003) y PICASSO Cruise (MD03, Shipboard Scientific Party, 2003) en los océanos Pacífico oriental y Atlántico occidental respectivamente. Los testigos estudiados en esta tesis se identifican por las estrellas amarillas de mayor tamaño (ODP 1240, 1241 y 1242, localizados en la cuenca de Panamá; y MD03-2616 localizado en la cuenca de la Guayana). Mapa de fondo tomado del Google Earth (2008; Image NASA, Image Digital Globe 2008). 
Estas secuencias son posteriormente integradas en cada testigo convirtiendo los datos de profundidad "mbsf" de cada pozo a datos de profundidad compuesta ("meters composite depth", mcd), utilizando el programa propuesto por el proyecto ODP (http://www-odp.tamu.edu/). La conversión a profundidad compuesta se realiza con el fin de reconstruir una sola secuencia sedimentaria y posteriormente establecer la escala de tiempo de cada testigo. La recuperación de secuencias sedimentarias completas se logra con la perforación de dos, tres o más pozos para cada testigo, de manera tal que se garantice la probabilidad de que los intervalos que se pierdan en un pozo puedan ser recuperados en otro adyacente. La revisión y la unificación de los pozos en cada testigo, se lleva a cabo por medio de un rastreo rápido empleando la susceptibilidad magnética o los registros del color o gamma natural, con muestreos de $5 \mathrm{~cm}$ de resolución; aunque en algunas ocasiones es necesario realizar integraciones en tiempo, cada segundo. Los testigos de la campaña ODP 202 registraron señales de susceptibilidad magnética muy bajas debido a su carácter litológico primordialmente biogénico. Por ello algunos testigos requirieron integraciones en tiempo más lentas, cada 10 segundos, y en otros casos se recurrió al uso de método que consiste en un rastreo multisensor-MST ("Multisensor Track"), el cual aunque es mucho más lento, permite la obtención datos de mejor calidad (http://wwwodp.tamu.edu/).

Las escalas de profundidad compuesta generalmente se expanden de entre el 10 al $20 \%$, debido al rebote elástico, la expansión de hidrocarburos volátiles (principalmente metano biogénico) y el ensanchamiento mecánico durante los procesos de extracción del sedimento. Se asume con antelación que esta expansión no afecta significativamente la absorción o pérdida del agua intersticial por unidad de masa de sedimento seco, permitiendo así que la estimación de las densidades de los sedimentos determinadas por las medidas de las masas (seca y húmeda) y de volumen, sean más compatibles con las medidas "in situ".

\subsubsection{Campaña PICASSO}

La campaña PICASSO se organizó dentro del marco del programa internacional IMAGES (International Marine Past Global Change Study) en dos expediciones, mar Caribe-cuenca de Cariaco; y Atlántico Norte. Durante la expedición del mar Caribe-cuenca de Cariaco se extrajeron 10 testigos entre los 70N y 170N (Fig. 4.1) mediante el sistema "Calypso". El objetivo científico general de la campaña fue recuperar testigos caracterizados por una alta tasa de sedimentación con el fin de evaluar los cambios paleoclimáticos y paleoceanográficos a escala orbital y 
milenaria, y su relación con los cambios geoquímicos ocurridos en esa región. Los períodos de tiempo de mayor interés científico en esta campaña comprenden el Holoceno y a más baja resolución, los últimos dos o tres ciclos climáticos, los cuales están vinculados a una serie de objetivos específicos que buscan (Shipboard Scientific Party, 2003):

a. Documentar los cambios ocurridos en la ruta de las corrientes de la Guayana y Caribe, las cuales llevan aguas cálidas desde el trópico hacia el Atlántico Norte a escala milenaria y sub-milenaria y su relación con la corriente del Golfo a altas latitudes.

b. Estudiar los cambios glaciales/interglaciales de la intensidad de los vientos sobre el Atlántico occidental y su impacto sobre los cambios en la humedad atmosférica en Centro América.

c. Analizar la migración latitudinal de la zona de convergencia de los vientos alisios (Intertropical Convergence Zone -ITCZ) en el Atlántico occidental, y la relación de estos cambios con la Oscilación del Sur.

Los testigos de la expedición mar Caribe-cuenca de Cariaco ofrecen un gran potencial para explicar el papel que juegan las regiones localizadas a bajas latitudes y los efectos que generan estas regiones en el clima de las altas latitudes o a nivel global. Los testigos de esta expedición fueron escogidos en parte para cumplir el programa científico de otros cruceros anteriores llevados a cabo por el Marion Dufresne, cuando la falta de tiempo en aquellos cruceros no permitió su extracción. Tal es el caso de los testigos del océano Atlántico occidental tropical y mar Caribe.

Entre los testigos extraídos de la expedición mar Caribe-cuenca de Cariaco, se encuentra el testigo MD03-2616, localizado en la cuenca de la Guayana y seleccionado en esta investigación como contribución a los objetivos planteados por la campaña PICASSO. La localidad de este testigo corresponde a una cuenca oceánica compleja, que desempeña un papel muy importante en la transferencia interlatitudinal de calor entre el trópico y el Atlántico Norte (Rühlemann et al., 2001).

La estrategia seguida para la recuperación de los testigos en la campaña PICASSO incluye la técnica "Calypso", la cual utiliza un tipo de pistón desarrollado a bordo del buque Marion Dufresne. Este pistón ayuda a mantener las condiciones de la presión litostática mientras se realiza la penetración del sacatestigos ("corer") a través de la fuerza gravitacional con el fin de 
minimizar la deformación y distorsión del sedimento. Se considera como el más práctico para la obtención de sondeos sedimentarios en el océano, y ha llegado a obtener sondeos continuos de más de $65 \mathrm{~m}$ de longitud. El sacatestigos desciende lentamente y una vez el contrapeso llega al fondo del océano, se libera el sacatestigos, el cual cae por la fuerza gravitacional. Este cortador empieza a penetrar por la velocidad gravitacional en el sedimento y efectúa la extracción. Cuando el sedimento ha sido extraído, se lleva a tubos plásticos de PVC los cuales son divididos en piezas de $1.5 \mathrm{~m}$ de longitud que posteriormente son cortadas en forma longitudinal. Cada par de piezas es marcado y una de ellas se deja como sección de trabajo y la otra queda como archivo.

\subsection{Descripción de los testigos}

\subsubsection{Testigo ODP 1240}

La secuencia sedimentaria extraída para el testigo 1240 se extiende a lo largo de 282.9 mcd superiores, los cuales comprenden el período que va desde el Plioceno Tardío hasta el Holoceno. Para ello fueron necesarios 4 pozos: A, B, C y D extraídos completamente con el sistema APC, de ellos el pozo A llegó hasta el basamento basáltico. Los sedimentos son esencialmente de origen biogénico mientras que los componentes siliciclásticos, especialmente arcillas, son raros (Mix et al., 2003). Esta secuencia sedimentaria consiste principalmente de fangos de nanofósiles con cantidades variables de diatomeas. Para el conjunto de sedimentos extraídos se definió una unidad litoestratigráfica dividida en tres subunidades, las cuales fueron diferenciadas esencialmente por las propiedades físicas y en menor medida por sus diferencias litológicas (Mix et al., 2003; Fig. 4.2; Tabla 4.1). El intervalo estudiado en esta investigación corresponde a los primeros $52.10 \mathrm{mcd}$, los cuales forman parte la Subunidad IA (Tabla 4.1; Fig. $4.2)$.

Los sedimentos de esta subunidad son primordialmente biogénicos, compuestos por fangos de nanofósiles con diatomeas y fangos de nanofósiles. Hacia el metro $\sim 34 \mathrm{mcd}$, se localiza el fango de nanofósiles con arcillas (y diatomeas), en el cual en alguna ocasión se incluye también un fango de nanofósiles con foraminíferos (y diatomeas). La alternancia entre fangos de nanofósiles con diatomeas y fangos de diatomeas con nanofósiles es muy común en los 100 mcd superiores; los cuales registran una bioturbación intensa, permitiendo la alternancia del color del sedimento 
entre oliva pálido y gris oliva suave. Los sedimentos biogénicos consisten principalmente de nanofósiles (40-80\%) y diatomeas (10-40\%), con pequeñas cantidades de foraminíferos $(0-15 \%)$.

\begin{tabular}{|c|c|c|c|c|}
\hline Subunidad & Profundidad & Edad & $\begin{array}{c}\text { Tasa de } \\
\text { sedimentación }\end{array}$ & $\begin{array}{c}\text { Propiedades físicas y características } \\
\text { litológicas }\end{array}$ \\
\hline IA & $0-142.2 \mathrm{mcd}$ & $0-1.7 \mathrm{Ma}$ & $\sim 80 \mathrm{~m} / \mathrm{Ma}$ & $\begin{array}{l}\text { Compuesta principalmente de un fango de } \\
\text { nanofósiles con altas abundancias relativas } \\
\text { de diatomeas. Está fuertemente bioturbada. }\end{array}$ \\
\hline IB & $142.2-206.4 \mathrm{mcd}$ & $1.7-2.2 \mathrm{Ma}$ & $120 \mathrm{~m} / \mathrm{Ma}$ & $\begin{array}{l}\text { Esta Subunidad está enriquecida en: } \\
\text { diatomeas, componentes silisiclásticos, TOC, } \\
\text { y pigmentos relacionados con la clorofila. Las } \\
\text { bandas de color oscuro-claro son comunes. } \\
\text { Bajos valores de densidad, susceptibilidad } \\
\text { magnética y color, altos valores del registro } \\
\text { de gamma natural. }\end{array}$ \\
\hline IC & $206.4-282.9 \mathrm{mcd}$ & $2.2-2.6 \mathrm{Ma}$ & $120 \mathrm{~m} / \mathrm{Ma}$ & $\begin{array}{l}\text { Compuesta principalmente de un fango de } \\
\text { nanofósiles con altas abundancias relativas } \\
\text { de diatomeas. Intensa bioturbación. La } \\
\text { arcilla, el vidrio volcánico y la micrita son } \\
\text { componentes minoritarios. Se incluyen } \\
\text { también } 5 \text { niveles de cenizas volcánicas, } 3 \text { de } \\
\text { las cuales son correlacionables en los pozos } \\
\text { A y B. }\end{array}$ \\
\hline
\end{tabular}

Tabla 4.1. Caracterización de las tres subunidades litoestratigráficas del testigo ODP 1240 (Mix et al., 2003)

Las espículas de esponjas, silicoflagelados, y radiolarios forman un 3\% del sedimento. Los materiales siliciclásticos son un componente minoritario y representan solo un $5 \%$. Debajo de los primeros $100 \mathrm{mcd}$, las diatomeas incrementan su abundancia relativa sobre los nanofósiles. También se registran tres niveles de cenizas volcánicas en la Subunidad IA las cuales muestran evidencias de bioturbación, su color varía entre marrón, gris y gris oscuro, y continenen vidrio vesicular. Entre los minerales accesorios se encuentran la biotita, el cuarzo, y los feldespatos y raras ocurrencias de clinopiroxeno y anfíboles. La micrita también se hace presente de manera minoritaria. La susceptibilidad magnética es baja pero relativamente más alta y más variable que en la Subunidad IB (Mix et al., 2003; Fig. 4.2). 

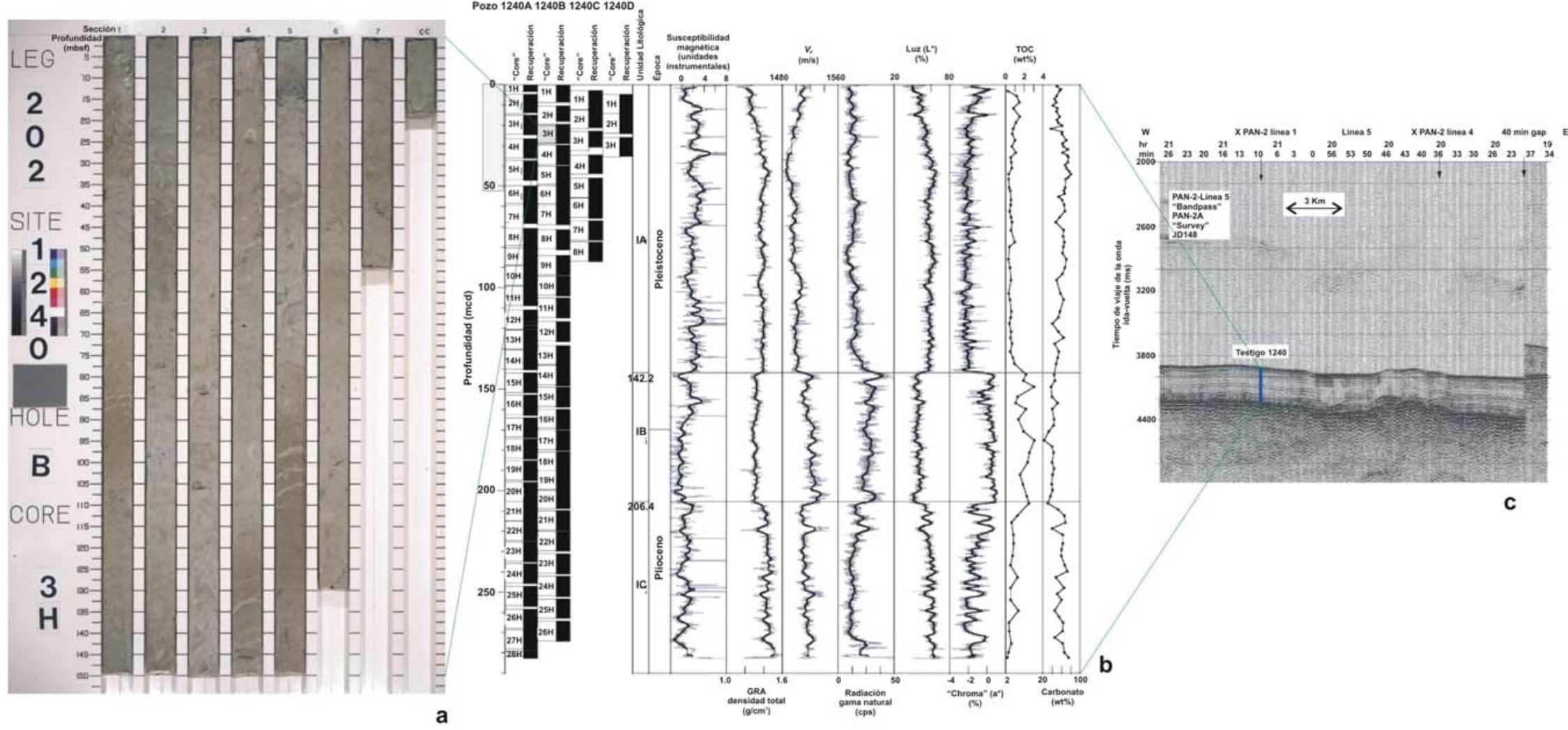

Fig. 4.2. Secuencia sedimentaria correspondiente al Testigo ODP 1240. a) Fotografía de la Unidad Litosedimentaria del "Core" 3H del Pozo $1240 B$ como ejemplo de lo que serían los demás "Cores" del testigo. Las unidades en profundidad corresponden a mbsf ("meters below sea floor", modificada de http://uww-odp.tamu.edu). b) Unidades litoestratigráficas y reconstrucción de la secuencia sedimentaria del Testigo 1240 empleando las propiedades físicas de los sedimentos. Las unidades en profundidad corresponden a mod ("meters composite depth", modificada de Mix et al., 2003). E cuadro gris localizado entre 0 y 52.10 mod, corresponde al intervalo estudiado para el testigo 1240 (los últimos 560 Ka). c) Perfíl sísmico del Testigo 1240 (Mix et al., 2003 ). 


\subsubsection{Testigo ODP 1241}

La sucesión sedimentaria recuperada para el testigo ODP 1241 tiene 447.06 mcd de longitud (Mioceno a Holoceno), la cual pudo extraerse a través de 3 pozos ( $A, B$ y $C$ ) con la técnica APC; adicionalmente, se utilizó la técnica XCB para los pozos A y B con el fin de lograr el objetivo en profundidad. La sucesión del testigo 1241 es esencialmente biogénica y consiste principalmente de un fango de nanofósiles, que en algunas ocasiones incluye intervalos con cantidades significativas de diatomeas y foraminíferos. Los componentes siliciclásticos están representados especialmente por arcilla. La variación relativa de estos componentes, junto con las propiedades físicas del sedimento, determinaron la división de esta unidad en tres subunidades litológicas (Tabla 4.2; Fig. 4.3).

\begin{tabular}{|c|c|c|c|c|}
\hline Subunidad & Profundidad & Edad & $\begin{array}{c}\text { Tasa de } \\
\text { sedimentación }\end{array}$ & $\begin{array}{c}\text { Propiedades físicas y características } \\
\text { litológicas }\end{array}$ \\
\hline IA & $0-51.55 \mathrm{mcd}$ & $\begin{array}{c}0-2.10 \\
\mathrm{Ma}\end{array}$ & $26 \mathrm{~m} / \mathrm{Ma}$ & $\begin{array}{l}\text { Compuesta principalmente de un fango de } \\
\text { nanofósiles con altas proporciones de } \\
\text { diatomeas y frecuente bioturbación. }\end{array}$ \\
\hline IB & $\begin{array}{l}51.55-318.44 \\
\mathrm{mcd}\end{array}$ & $\begin{array}{c}2.10-9 \\
\mathrm{Ma}\end{array}$ & $36-67 \mathrm{~m} / \mathrm{Ma}$ & $\begin{array}{l}\text { Compuesta principalmente de fango de } \\
\text { nanofósiles, con contribuciones variables de } \\
\text { diatomeas y foraminíferos. Se incluyen } \\
\text { adicionalmente } 23 \text { horizontes volcaniclásticos. El } \\
\text { perfil de color incrementa sus valores hacia el } \\
\text { tope de la Subunidad. La base de la Subunidad } \\
\text { IB está definida por una capa correlacionable de } \\
\text { ceniza volcánica a los } 318.44 \mathrm{mcd} \text {. }\end{array}$ \\
\hline IC & $\begin{array}{l}318.44-447.06 \\
\mathrm{mcd}\end{array}$ & $9-11 \mathrm{Ma}$ & $50 \mathrm{~m} / \mathrm{Ma}$ & $\begin{array}{l}\text { Compuesta principalmente por arcilla y por un } \\
\text { fango de nanofósiles con diatomeas y un fango } \\
\text { de diatomeas laminadas. Se incluyen también } \\
29 \text { horizontes volcaniclásticos. Registra valores } \\
\text { de susceptibilidad magnética y gamma natural } \\
\text { relativamente más altos que la Subunidad IB. }\end{array}$ \\
\hline
\end{tabular}

Tabla 4.2. Caracterización de las tres subunidades litoestratigráficas del testigo ODP 1241 (Mix et al., 2003)

Dentro de las subunidades litológicas se intercalan ocasionalmente algunas capas de cenizas volcánicas y lapilli, registrando así la historia del volcanismo de Centroamérica, Suramérica 0 Galápagos. Sobre el nivel situado a una profundidad entre 420-440 mcd se presenta un intervalo 


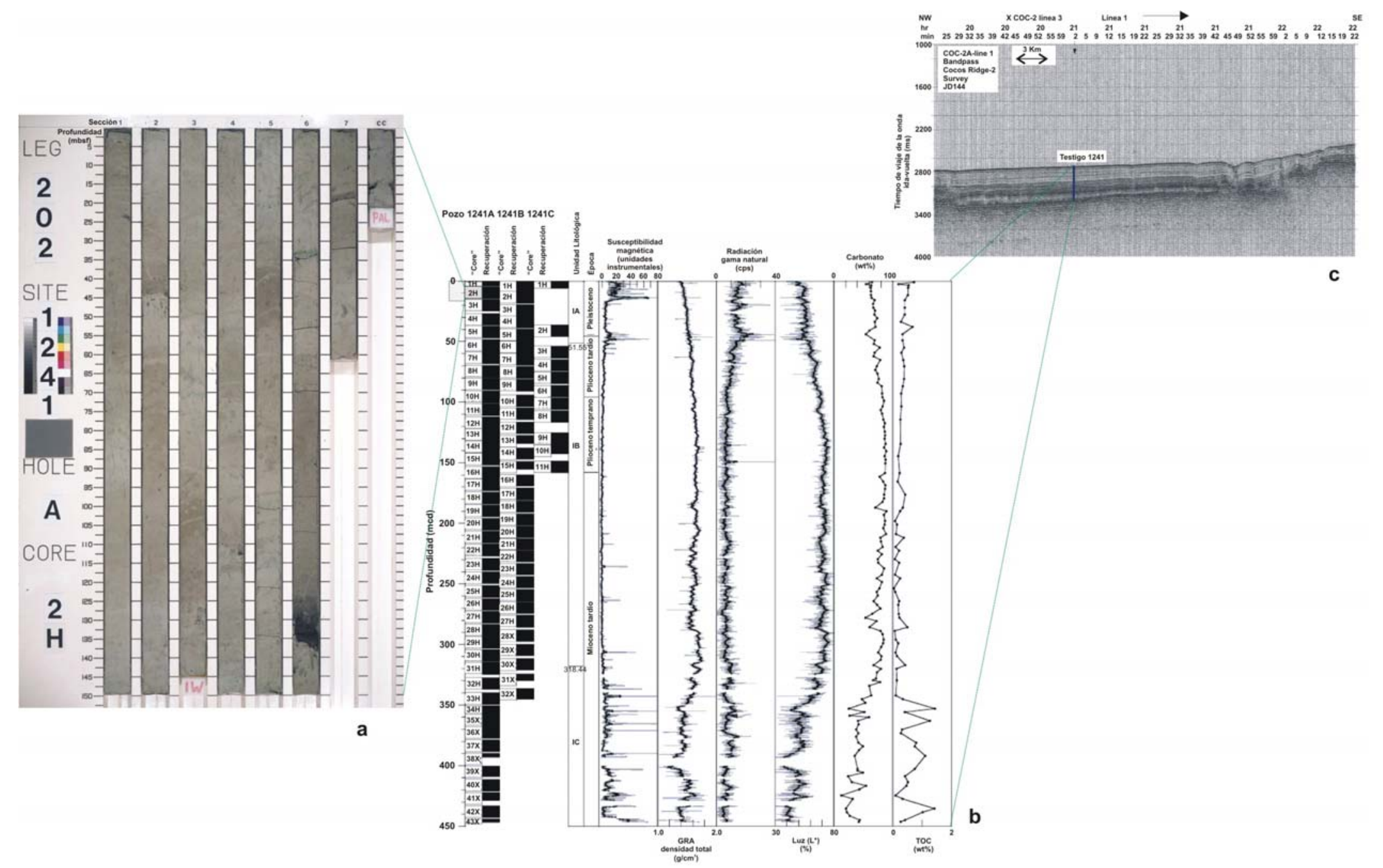

Fig. 4.3. Secuencia sedimentaria correspondiente al Testigo ODP 1241. a) Fotografía de la Unidad Litosedimentaria del "Core" $2 \mathrm{H}$ del Pozo $1241 \mathrm{~A}$ comoejemplo de lo que serían los demás "Cores" del testigo. Las unidades en profundidad corresponden a mbsf ("meters below sea floor", modificada de http://unw-odp.tamu.edu). b) Unidades litoestratigráficas y reconstrucción de la secuencia sedimentaria del Testigo 1241 empleando las propiedades físicas de los sedimentos. Las unidades en profundidad corresponden a mcd ("meters composite depth", modificada de Mix et al., 2003). $\boxminus$ cuadro gris localizado entre 0 y 17 mcd, corresponde al intervalo estudiado para el testigo 1241 (los últimos 830 Ka). c) Perfíl sísmico del Testigo 1241 (Mix et al., 2003). 
laminado que corresponde a un fango de diatomeas. El basamento basáltico infrayace directamente la secuencia pelágica (Mix et al., 2003). La secuencia sedimentaria estudiada en esta tesis se localiza dentro de la Subunidad IA y corresponde al intervalo entre 0 y 17 mcd (Tabla 4.2; Fig. 4.3). La Subunidad IA está compuesta por un fango de nanofósiles y foraminíferos con arcillas (foraminíferos, 25-45\% y nanofósiles, 30-60\%). Los componentes silíceos biogénicos están en menor proporción (diatomeas $<12 \%$, y esponjas, espículas, radiolarios y silicoflagelados suman $\leq 7 \%$ ). La abundancia relativa de los nanofósiles y las diatomeas se incrementa con la profundidad, mientras los componentes siliciclásticos y los foraminíferos decrecen. La variación del color se alterna entre un gris oliva a un gris oliva claro y un oliva pálido. La bioturbación es frecuente en la Subunidad IA. Los horizontes volcaniclásticos también están presentes, incluyen cenizas volcánicas y lapilli. Las cenizas contienen vidrio claro y marrón, minerales máficos y cuarzo. Las propiedades físicas de esta subunidad son muy variables; la señal de susceptibilidad magnética es relativamente intensa, y la variación de gamma natural es diáfana y también registra valores altos, mientras que la señal de color es relativamente baja $(<60 \%)$, diferenciando así la Subunidad IA de las demás subunidades. La base de la unidad se estableció en el metro 51.6 mcd a través de la correlación de una capa de ceniza volcánica (Mix et al., 2003; Tabla 4.2; Fig. 4.3).

\subsubsection{Testigo ODP 1242}

La secuencia sedimentaria recuperada corresponde a los 287.74 mcd superiores correspondientes al intervalo comprendido entre el Mioceno medio hasta el Holoceno. La reconstrucción de esta secuencia sedimentaria se realizó con base en la extracción de 4 pozos (A, B, C y D; Fig. 4.4) con la técnica APC; la extracción final de los pozos A y B se realizó con la técnica XCB. La secuencia extraída fue dividida en dos unidades separadas por un hiato prominente entre los 2.5-12 Ma. La Unidad I se extiende desde el Plioceno superior hasta el Holoceno, mientras que la Unidad II lo hace en el Mioceno medio. El hiato está caracterizado por un cambio de color muy definido de verde oliva grisoso (Unidad I) a un marrón pálido (Unidad II). El intervalo estudiado comprende los $108.01 \mathrm{mcd}$ superiores de la Unidad I. La litología principal de la Unidad I incluye los fangos de nanofósiles con arcillas. Los nanofósiles son el componente biogénico dominante $(30-70 \%)$, siendo frecuentemente diluidos por el material siliciclástico. La abundancia de nanofósiles se incrementa en profundidad paralelamente a la micrita. Los foraminíferos $(\sim 4-12 \%)$ y las diatomeas $(<<10 \%)$ están presentes en cantidades minoritarias, pero pueden llegar al 20\% ( 155 mcd) y al 10\% (100-140 mcd) respectivamente. Los radiolarios, espículas y silicoflagelados apenas pueden llegar al $2 \%$. Los componentes siliciclásticos (20-60\%) están dominados por las arcillas con pocas cantidades de minerales tamaño limo o arena (cuarzo y feldespato) a través de la Unidad. Los siliciclastos 
aumentan su abundancia relativa entre los 10-25 mcd y disminuyen a partir de los 180 mcd hacia la base de la unidad. Los minerales autigénicos también se encuentran en bajas proporciones pero incrementan en profundidad. El vidrio volcánico igualmente es un componente minoritario en la unidad. El color a veces puede presentar cambios gradacionales entre tonos de verde oliva grisoso. La bioturbación es moderada. Entre los 205 y 215 mcd se registra un nivel oscuro de diatomeas. Hacia la base de la unidad se encuentran granos gruesos negros compuestos de arcilla rica en pirita; y glauconita. Adicionalmente se detectaron 45 horizontes de cenizas volcánicas, 18 de las cuales pueden correlacionarse entre los pozos, y están presentes en los 278 mcd superiores. Como la concentración de carbonato aumenta con la profundidad, las señales de color y densidad aumentan en profundidad, mientras que los registros de susceptibilidad magnética y gamma natural lo hacen hacia el tope de la unidad, excepto en los niveles de cenizas donde su señal crece de manera evidente (Tabla 4.3; Fig. 4.4).

\begin{tabular}{|c|c|c|c|c|}
\hline Unidad & Profundidad & Edad & $\begin{array}{c}\text { Tasa de } \\
\text { sedimentación }\end{array}$ & $\begin{array}{c}\text { Propiedades físicas y características } \\
\text { litológicas }\end{array}$ \\
\hline I & $0-280.63 \mathrm{mcd}$ & $0-2.5 \mathrm{Ma}$ & $60-200 \mathrm{~m} / \mathrm{Ma}$ & $\begin{array}{l}\text { Sedimentos hemipelágicos homogéneos, } \\
\text { principalmente de grano fino. Altas proporciones } \\
\text { de carbonato y baja abundancia relativa de } \\
\text { siliciclastos caracterizan la base de la Unidad } \\
\text { (Plioceno superior), mientras que la baja } \\
\text { concentración de carbonato y el enriquecimiento } \\
\text { en componentes siliciclásticos se dirigen hacia el } \\
\text { tope de la Unidad (Pleistoceno superior). }\end{array}$ \\
\hline II & $280.63-287.74 \mathrm{mcd}$ & $12-13 \mathrm{Ma}$ & $7 \mathrm{~m} / \mathrm{Ma}$ & $\begin{array}{l}\text { Esencialmente sedimentación biogénica } \\
\text { compuesta principalmente por nanofósiles ( } 60- \\
70 \%) \text { y diatomeas }(\sim 10-20 \%) \text {. Los foraminíferos, } \\
\text { espículas, radiolarios y silicoflagleados con } \\
\text { componentes biogénicos minoritarios. Las señales } \\
\text { de susceptibilidad magnética y gamma natural } \\
\text { cambian abruptamente y decrecen en profundidad, } \\
\text { junto con los componentes siliciclásticos, mientras } \\
\text { que el color se incrementa. }\end{array}$ \\
\hline
\end{tabular}

Tabla 4.3. Caracterización de las dos unidades litoestratigráficas del testigo ODP 1242 (Mix et al., 2003) 


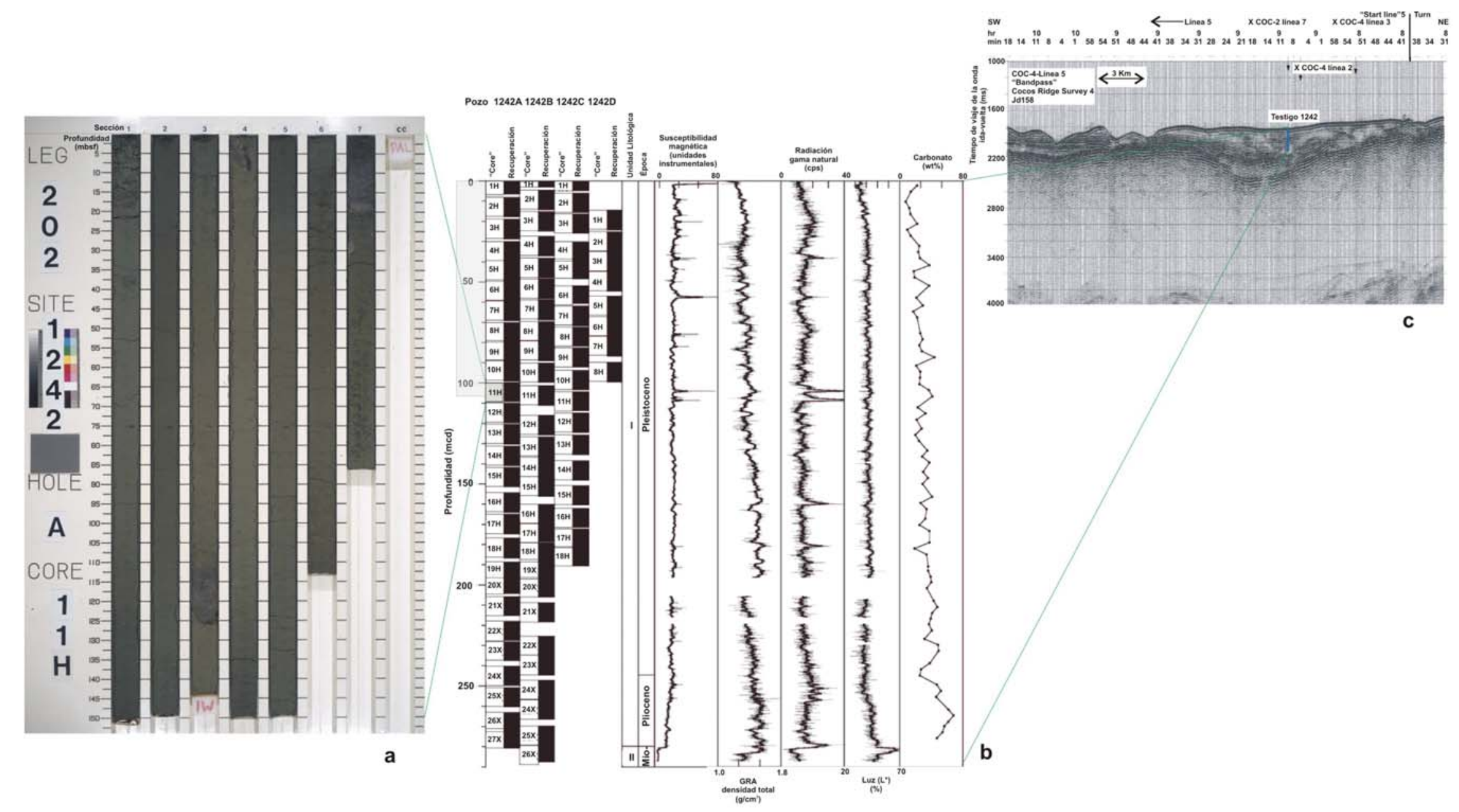

Fig. 4.4. Secuencia sedimentaria correspondiente al Testigo ODP 1242. a) Fotografía de la Unidad Litosedimentaria del "Core" 11H del Pozo 1242A como ejemplo de lo que serían los demás "Cores" del testigo. Las unidades en profundidad corresponden a mbsf ("meters below sea floor", modificada de http://huw-odp.tamu.edu). b) Unidades litoestratigráficas y reconstrucción de la secuencia sedimentaria del Testigo 1242 empleando las propiedades físicas de los sedimentos. Las unidades en profundidad corresponden a mcd ("meters composite depth", modificada de Mix et al., 2003). $\boxminus$ cuadro gris localizado entre O y 108.10 mcd, corresponde al intervalo estudiado para el testigo 1242 (los últimos 925 Ka). c) Perfíl sísmico del Testigo 1242 (Mix et al., 2003. 


\subsubsection{Testigo MD03-2616}

La secuencia sedimentaria tiene una longitud de $39 \mathrm{~m}$ (0 400 Ka), de los cuales se estudiaron los primeros $18 \mathrm{~m}$ que equivalen al último ciclo climático (0 150 Ka; Fig. 4.5). La litología hemipelágica del testigo comprende cantidades variables de arcillas y limos de foraminíferos planctónicos y bentónicos, y nanofósiles de color verde oliva a gris distribuidos homogéneamente a lo largo de la secuencia sedimentaria, con pequeñas intervenciones de niveles bioturbados y de cambio de color. La proporción del componente biogénico carbonatado es mayor entre la base del testigo y los $125 \mathrm{Ka}$, a partir de los cuales este componente disminuye y la señal de la susceptibilidad magnética se incrementa. Los restos orgánicos son abundantes y se presentan como moteados de color negro, también son abundantes los tallos vegetales, los fragmentos de briozoos y de moluscos (Shipboard Scientific Party, 2003).

\subsection{Téc nicas de preparación de las muestras}

\subsubsection{Preparación de "láminas" para el análisis de cocolitóforos}

Existen diversas técnicas de preparación del sedimento para la observación y el estudio de los nanofósiles calcáreos mediante el empleo del microscopio óptico o electrónico, las cuales han sido propuestas por diversos autores (Backman y Shackleton, 1983; Wei, 1988; Baumann, 1990; Beaufort, 1991; Samtleben y Schröder, 1992; Okada, 1992; Henrikson, 1993; Andruleit, 1996; Flores y Sierro, 1997) con el ánimo de optimizar el tiempo y el procedimiento que lleva al objetivo propuesto en los estudios micropaleontológicos realizados.

Entre las técnicas más utilizadas están por ejemplo, la técnica del frotis ("smear slides”, Backman y Shackleton, 1983), de decantación (Flores y Sierro, 1997) y de filtración (Backman y Shackleton, 1983; Andruleit, 1996). La técnica del frotis es la más sencilla y rápida porque permite el procesamiento de un enorme número de muestras en corto tiempo, resulta bastante práctica en los estudios puramente bioestratigráficos, y es la que se sigue en las campañas oceanográficas; sin embargo, esta técnica no permite un procesamiento reproducible en cada muestra ni el análisis cuantitativo de los taxones identificados. Las técnicas de decantación y de filtración por el contrario, ofrecen un procesamiento reproducible en las muestras a analizar y además resultan muy útiles a la hora de establecer análisis cuantitativos de la asociación. Estas 

Investigación.

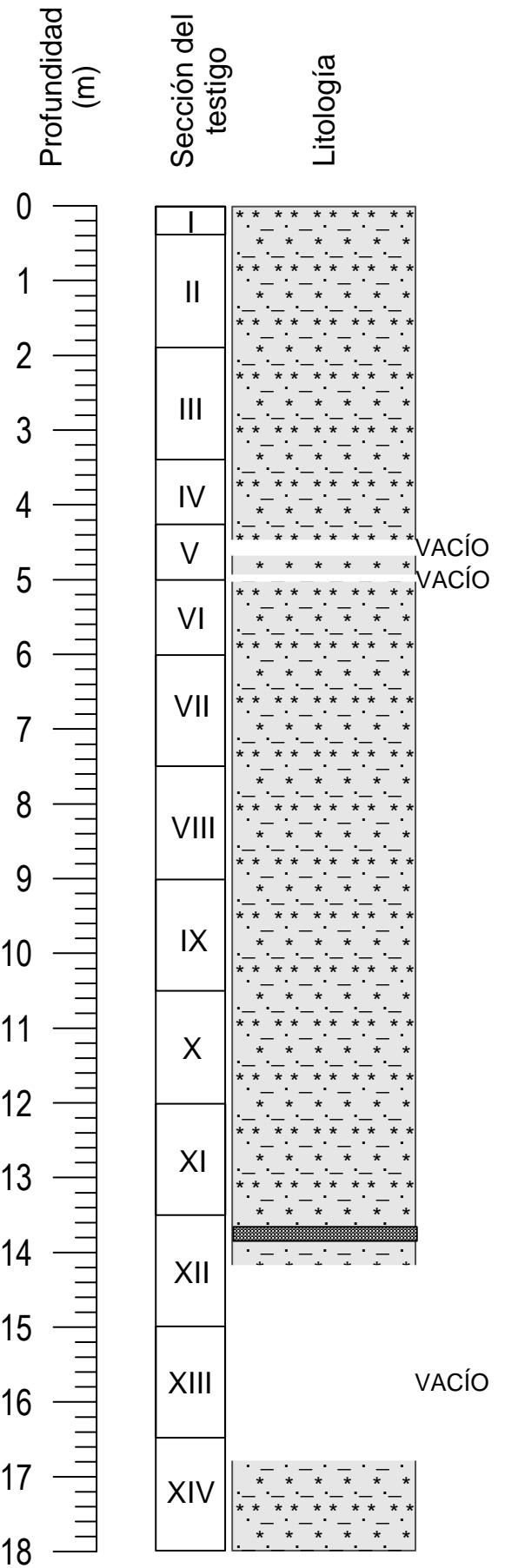

Arcilla limosa de foraminíferos y nanofósiles, color verde oliva a gris cm 16-19: nivel bioturbado de arcilla de nanofósiles, color verde Arcilla de foraminíferos y nanofósiles de color verde cm 190-240: suave bioturbación

Arcilla limosa de foraminíferos y nanofósiles de color verde oliva $\mathrm{cm}$ 340-370: manchas negras dispersos

Arcilla limosa de foraminíferos-nanofósiles, color verde oliva, manchas negras dispersas Arcilla limosa de nanofósiles, color verde oliva, sin bioturbación Arcilla limosa de nanofósiles muchas manchas negras

Arcilla limosa de nanofósiles, manchas negras y pirita cm 628-637: Arcilla limosa de nanofósiles, color verde oliva a gris; "burrows" cm 637-665: Arcilla limosa de nanofósiles, color verde oliva cm 665-710: Arcilla limosa de foraminíferos-nanofósiles, color verde oliva cm 710-718: Arcilla limosa de nanofósiles, color gris oliva

Arcilla limosa de nanofósiles, color gris oliva abundantes manchas negras

cm 910-940: "Burrows"

Arcilla de nanofósiles gris oliva abundantes manchas negras

Arcilla limosa de nanofósiles gris oliva pocos "burrows" y pirita abundantes manchas negras

Arcilla limosa de nanofósiles, color gris oliva abundantes manchas negras algunos nódulos de pirita cm 1350-1365: Arcilla limosa de nanofósiles, color gris oliva cm 1365-1375: arenas de foraminíferos, color gris cm 1375-1405: Arcilla limosa de nanofósiles. color verde oliva

Arcilla limosa de foraminíferos-nanofósiles, color verde oliva algunas capas ricas en foraminíferos, abundantes manchas negras Algunas concreciones de pirita, generalmente rellenando los "burrows"

Fig. 4.5. Descripción sedimentológica los $18 \mathrm{~m}$ superiores del testigo MD03-2616. El tope de los intervalos "vacíos" se ajusta perfectamente con la base del nivel que está inmediatamente sobre él; y la base de los intervalos "vacíos" se ajusta perfectamente con el techo del nivel que está inmediatamente debajo de él (Modificada de Shipboard Scientific Party, 2003). 


\section{La técnica de Decantación}

Esta técnica fue propuesta por Flores y Sierro (1997) y fue empleada en la preparación de todas las muestras de los testigos estudiados con el objetivo de realizar el recuento de los especímenes en cada muestra y así establecer los análisis cuantitativos de cocolitos que se describirán más adelante. El análisis de las muestras preparadas con la técnica de decantación requiere del empleo del microscopio óptico, y para ello se utilizó el Microscopio LEICA DMRXE en los Laboratorios de Micropaleontología de la Universidad de Salamanca.

La metodología se inicia con el peso previo de $0.2 \mathrm{~g}$ de sedimento seco para cada muestra, los cuales son diluidos en $10 \mathrm{ml}$ de agua tamponada (agua destilada más $\mathrm{Na}_{2} \mathrm{CO}_{3}$ y $\mathrm{NaHCO}_{3}$ ) empleando el ultrasonidos. El uso del agua tamponada permite conservar el pH apropiado del agua destilada en la que se va a realizar el procedimiento de preparación. De cada muestra diluida, se extraen $100 \mu$ l que se agregan a la solución de agua tamponada con Gelatina de Oro contenida en placas Petri de diámetro conocido a las que previamente se les ha incorporado un cubreobjetos y además han sido identificadas con el nombre de cada muestra. La Gelatina de Oro se prepara con antelación y su uso evita la tensión superficial de las partículas en el borde de la placa durante el proceso de decantación. La mezcla resultante contenida en las placas Petri es homogenizada cuidadosamente utilizando una pipeta, con el fin de facilitar la distribución uniforme de todas las partículas de sedimento. Una vez todas las muestras se han homogenizado, se ponen a decantar entre 12 y 24 horas sobre una superficie horizontal, a temperatura ambiente evitando todo tipo de vibraciones o contaminación.

Pasado el tiempo de decantación, el exceso de humedad de cada placa Petri se extrae por capilaridad, cuidando de no rozar el cubreobjetos que contiene el material decantado. Posteriormente, este cubreobjetos se deja secar totalmente a temperatura ambiente dentro de la placa Petri. Cuando el cubreobjetos está seco se lleva a una placa térmica a $45^{\circ} \mathrm{C}$, se le agrega dos gotas de Bálsamo de Canadá disuelto con xileno al $50 \%$, y se pega al portaobjetos que debe estar previamente etiquetado con el nombre de la muestra. Por último, la lámina recién preparada se deja reposar durante un par de horas en la plancha térmica con el fin de extraer todo el xileno y facilitar el secado; y luego se deja a temperatura ambiente hasta que seque totalmente. La reproducibilidad de esta técnica depende de la correcta homogenización de la mezcla y con ella de la uniformidad del sedimento decantado sobre el cubreobjetos. 


\section{La técnica de filtración}

Esta técnica fue empleada originalmente por Mclntyre, fue descrita por Backman y Schackleton (1983) y luego fue adaptada por Andruleit (1996). Es una técnica rápida, sencilla, permite el procesamiento y la reproducibilidad de gran número de muestras y la observación de cocolitos bajo los microscopios óptico y electrónico. La metodología que se siguió corresponde a la metodología propuesta por Andruleit (1996) la cual fue aplicada en los laboratorios de Paleontología de la Universidad de Kiel bajo la dirección del Dr. Hanno Kinkel, con el objetivo de examinar las muestras críticas de los tres sondeos del Pacífico oriental tropical (ODP 1240, 1241 y 1242) mediante el empleo de técnicas de Microscopía Electrónica de Barrido ("Scanning Electron Microscope", SEM) y fotografía exhaustiva de taxones para su catalogación y análisis de preservación con más precisión. No obstante, esta técnica puede ser empleada igualmente para el análisis bajo el microscopio óptico.

El procedimiento en esta técnica requiere el peso inicial de $0.06 \mathrm{~g}$ de sedimento seco para cada muestra y su dilución de agua tamponada empleando el ultrasonidos. Posteriormente se agrega medio litro de agua tamponada a cada muestra diluida y se agita manualmente hasta lograr la homogenización. Cada muestra diluida se divide en 10 alícuotas iguales (1/10) utilizando una máquina "rotary splitter", a 40 ó 50 revoluciones por minuto. De estas 10 alícuotas se elige una de ellas para repetir el paso anterior en la "rotary splitter" (1/100). Nuevamente se elige una segunda alícuota (1/100) que se filtra al vacío sobre un filtro de membrana de policarbonato (diámetro $50 \mathrm{~mm}$ y tamaño de poro $0.40 \mu \mathrm{m}$ ). Una vez haya ocurrido el proceso de filtración al vacío, la membrana de policarbonato se lleva a una placa Petri, se tapa y se seca lentamente en la estufa a $40^{\circ} \mathrm{C}$ durante un tiempo comprendido entre 6 a 12 horas. Cuando la membrana está seca se corta una pequeña pieza (alrededor de $1 \mathrm{~cm}^{2}$ ), la cual se lleva a un soporte especial técnicamente llamado "stub" y se cubre con una capa fina de oro-paladio mediante un evaporador al vacío. En este instante la muestra queda lista para realizar las observaciones pertinentes en el microscopio electrónico.

Al igual que en la técnica de decantación, la reproducibilidad de este método depende de la homogeneidad de las partículas sobre el filtro de policarbonato. Es adecuada para el cálculo de abundancias absolutas y relativas de cocolitos. Permite la comparación de datos obtenidos a través de la observación del plancton, trampas de sedimentos, y sedimentos con base en recuentos de unidades de cocosferas (de acuerdo a Samtleben y Schröder, 1992), y para el cálculo de flujos de cocolitóforos/cocolitos y tasas de acumulación. 


\subsubsection{Preparación de muestras para el análisis de isótopos de oxígeno en foraminíferos}

Inicialmente, se pesan entre 10 y $20 \mathrm{~g}$ de sedimento, los cuales se dejan en reposo en agua destilada durante 24 horas para facilitar la disgregación de arcillas (el intervalo de muestreo para el registro de $\delta^{18} \mathrm{O}$ en esta tesis fue establecido cada $3 \mathrm{~cm}$ que corresponden a una resolución de entre 100 a 700 años). Posteriormente, este sedimento es lavado y tamizado separando la fracción $>63 \mu \mathrm{m}$. Esta fracción de sedimento húmedo se lleva al horno a una temperatura de $\sim 45^{\circ} \mathrm{C}$, y una vez seca se vuelve a tamizar con el fin de separar las tallas entre 63-150 $\mu \mathrm{m}$ y $>150 \mu \mathrm{m}$, las cuales son pesadas y almacenadas separadamente. A continuación, la fracción $>150 \mu \mathrm{m}$ se divide en dos partes iguales con un microcuateador ("microsplitter") con el fin de separar la porción destinada a los análisis cuantitativos de foraminíferos (la cual no fue analizada en este estudio) y la porción destinada a los análisis geoquímicos.

La selección de los ejemplares de interés se restringió a la fracción de 300-350 $\mu \mathrm{m}$ con el fin de limitar los efectos ontogenéticos y de cuidar que los ejemplares seleccionados corresponden a un mismo morfotipo. Entre 38 a 60 ejemplares fueron seleccionados para la especie Uvigerina peregrina. Esta especie fue seleccionada debido a su gran abundancia y su registro siempre continuo a lo largo del testigo, teniendo en cuenta la preferencia por las conchas mejor preservadas y las más limpias. Una vez los ejemplares han sido separados se procedió a la fragmentación de las conchas de los foraminíferos con la finalidad de eliminar cualquier fase de contaminación en su interior a lo largo del proceso de limpieza. Los ejemplares fragmentados se mezclan entre si para cada muestra y de esa mezcla se separa una tercera parte para el análisis de isótopos estables y dos terceras partes para otros análisis geoquímicos.

La limpieza de las conchas fragmentadas se lleva a cabo con dos enjuagues de metanol agitando con un sistema de utrasonidos durante algunos segundos con la finalidad de remover la fracción arcillosa. Los restos de metanol se eliminan posteriormente con un enjuague de agua milli-Q agitando nuevamente con el ultrasonidos durante breves segundos. $Y$ finalmente, la muestra se deja secando a temperatura ambiente durante una noche.

Aparentemente las muestras no tenían mucha influencia de contaminación de materia orgánica. Por ello se evitó cualquier otro proceso químico que pudiera interferir en los análisis de los isótopos estables. 


\subsection{Téc nicas de cuantíficación}

\subsubsection{Cálculo de la abundancia absoluta y estimación del flujo de cocolitos y del carbonato aportado}

Los cálculos de abundancias absolutas (cocolitos por gramo de sedimento seco) y relativas (\%) se basaron en el recuento de 500-600 cocolitos. Este número de cocolitos establece que todos los cocolitos con abundancia relativa mayor al $1 \%$ fueron estimados con una probabilidad de error de 0.005 (Dennison y Hay, 1967). La estimación de la abundancia absoluta fue calculada a partir del método propuesto por Flores y Sierro (1997):

$$
N=\frac{\left(n^{*} R^{2} * V\right)}{r^{2} * g * v}, \text { donde: }
$$

$N$ : abundancia absoluta (cocolitos $\left.{ }^{*} \mathrm{~g}^{-1}\right)$

$R$ : radio de cada placa Petri utilizada

$V$ : volumen de agua tamponada añadida al sedimento seco en los botes de $(\mu l)$

r: número de cocolitos contados por cuadrante

$g$ : peso del sedimento seco para cada muestra $(\mathrm{g})$

$v$ : volumen de la mezcla que se homogenizó en cada placa petri $(\mu l)$

El cálculo de las abundancias absolutas permite calcular la cantidad de cocolitos acumulados por unidad de área $\left(\mathrm{cm}^{2}\right)$ y de tiempo (Ka) (Mayer et al., 1992; Flores y Sierro, 1997). La tasa de acumulación de cocolitos ha sido muy utilizada como indicador de paleoproductividad, pues los máximos en la abundancia de cocolitos se relacionan con altas tasas de producción en las aguas superficiales.

$$
N A R=N * d * s \text {, donde: }
$$

NAR: flujo de cocolitos ("nannofossil accumulation rate", cocolitos $^{*} \mathrm{Cm}^{-2 *} \mathrm{Ka}^{-1}$

N: cocolitos/g

$d$ : densidad del sedimento seco $\left(\mathrm{g}^{*} \mathrm{Cm}-{ }^{3}\right)$

s: tasa de sedimentación lineal $\left(\mathrm{cm}^{*} \mathrm{Ka}^{-1}\right)$.

Los datos de abundancia absoluta fueron convertidos a masa de cocolitos con base en las estimaciones propuestas por Young y Ziveri (2000). En cada especie, existe un amplio rango de tamaños de cocolitos, y en consecuencia una gran variación en la estimación de la masa (Young y Ziveri, 2000). Por ello, las tallas de los taxones se separaron en los rangos más frecuentes con 
el fin de obtener una estimación más aproximada del carbonato aportado por ellos. Las estimaciones de la masa de los cocolitos son muy fáciles de obtener, sólo se debe contar con datos previos sobre la constante relacionada con la forma de cada especie, la longitud promedio de las especies y la densidad de la calcita. Las estimaciones de la masa son muy útiles en los estudios paleoecológicos, biogeográficos o de procesos de sedimentación, y doblemente útil en el caso de los cocolitos, considerados como uno los mayores aportadores de carbonato al fondo oceánico (Young y Ziveri, 2000).

$$
m=K_{s} * l^{3} * N * \delta, \text { donde: }
$$

m: masa de carbonato aportado por cocolitos $\left(\mathrm{pg}^{*} \mathrm{~g}^{-1}\right)$

Ks: Constante única para cada especie, la cual depende de la forma

I: longitud media del cocolito

$N$ : abundancia absoluta (cocolitos ${ }^{*} \mathrm{~g}^{-1}$ )

$\delta$ : densidad de la calcita $\left(2.7 \mathrm{pg}^{\star} \mu \mathrm{m}-{ }^{3}\right)$

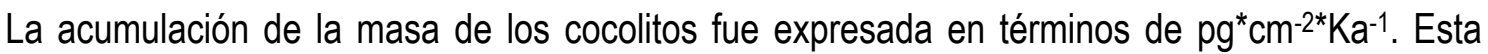
estimación ha sido propuesta en esta tesis como un indicador en el estudio de la paleoproductividad, el cual sugiere que una producción alta de cocolitos en el océano superficial es susceptible de favorecer altas tasas de acumulación de carbonato.

$$
C A R=m * d * s, \text { donde: }
$$

CAR: flujo de carbonato de cocolitos ("carbonate accumulation rate"; cocolitos ${ }^{*} \mathrm{~cm}^{-2 *} \mathrm{Ka}^{-1}$ )

m: masa

$N$ : cocolitos ${ }^{*} \mathrm{~g}^{-1}$

$d$ : densidad del sedimento seco $\left(\mathrm{g}^{*} \mathrm{~cm}-{ }^{3}\right)$

$s:$ tasa de sedimentación lineal $\left(\mathrm{cm}^{*} \mathrm{Ka}^{-1}\right)$

\subsubsection{Cálculo del índice $\mathbf{N}$}

El índice $\mathrm{N}$ es un índice de paleoproductividad, por ello vinculado a las fluctuaciones de la nutritermoclina Flores et al. (2000). El índice $N$ varía entre 0 y 1 y establece la relación entre los habitantes de la zona fótica superior (Gephyrocapsa oceanica, small Noelaerhabdaceae, Emiliania huxleyi) y los habitantes de la zona fótica inferior (Florisphaera profunda) (modificado de Flores et al., 2000). 


$$
N=\frac{\text { G.oceanica }+ \text { smallNoerlabdaceae }+ \text { E.huxleyi }}{\text { G.oceanica }+ \text { smallNoerlabdaceae }+ \text { E.huxleyi }+ \text { F.profunda }}
$$

Un índice cercano a 1 indica alta paleoproductividad, la cual está relacionada con una nutritermoclina somera y con la intensificación de las células de surgencia. Un índice $\mathrm{N}$ cercano a 0 , indica el descenso de la paleoproductividad y con ella la profundización de la nutri-termoclina y la estratificación del océano superior.

\subsubsection{Deteminación del grado de preservación del carbonato}

El carbonato biogénico es el principal componente sedimentario de las secuencias estudiadas, y los cocolitos generalmente dominan el carbonato biogénico en los sedimentos. La preservación influye significativamente en la distribución y composición de la flora y fauna calcárea en los océanos. Por ello es importante comprender la influencia de la disolución en las reconstrucciones paleoambientales y paleoclimáticas. El estado de preservación del carbonato en los testigos del Pacífico fue evaluado a través de la escala cualitativa sugerida por Roth y Thierstein (1972) y Flores y Marino (2002); y a través de las estimaciones del índice CEX' (Boeckel y Baumann, 2004).

La escala usada por Roth y Thierstein (1972) y Flores y Marino (2002) establece varios niveles de preservación, los cuales fluctúan entre: Buena ("good", G) indica que todas las estructuras y formas se conservan perfectamente; Moderada ("moderate", M) indica que alguna característica morfológica se ha alterado por disolución o fragmentación pero todos los ejemplares pueden identificarse a nivel de especie e incluso variedad de especie; Pobre ("poor", P) indica una disolución-fragmentación severas y por ello la identificación se da a nivel de género; Estéril ("barren", B) indica que no hay ningún registro de ejemplares de cocolitóforos. Adicionalmente, se establecieron tres escalas intermedias preservación buena a moderada, moderada a pobre y pobre a estéril, las cuales indican características de disolución intermedias. Esta escala se realizó a partir de la observación del estado de los ejemplares más delicados (por ejemplo los "small" Noelaerhabdaceae; Thierstein, 1980; entre otros) y la presencia o ausencia de estructuras frágiles (áreas centrales, puentes, bordes de los escudos). 
El índice CEX' fue propuesto originalmente por Dittert et al. (1999) y luego fue modificado por Böeckel y Baumann (2004). El índice CEX’ establece la relación de los taxones más delicados como son Emiliania huxleyi y los "small Noelaerhabdaceae" (excluyendo las ejemplares identificados como Gephyrocapsa aperta) y un taxón fuertemente calcificado, Calcidiscus leptoporus, caracterizado por ser resistente a la disolución y fragmentación. Valores del índice cercanos a 1 indican buena preservación, y valores cercanos a 0 indican un deterioro de la preservación.

En general los cocolitos revelan buena a moderada preservación en los testigos océanicos ODP 1240 y 1241. En los testigos costeros, ODP 1242 y MD03-2616, la preservación puede variar de buena a pobre, especialmente en el testigo MD03-2616, en el cual pudieron observarse intervalos muy bien preservados e intervalos estériles de cocolitos.

\subsection{Cronología}

El modelo de edad de los diferentes testigos del Pacífico oriental tropical están apoyados en la estratigrafía isotópica del $\delta^{180}$ del foraminífero planctónico Globigerinoides ruber (blanco) y los datos paleomagnéticos aportados por otros grupos científicos; y en la bioestratigrafía (biocronología) de cocolitóforos para los últimos $925 \mathrm{Ka}$, llevada a cabo en esta Tesis Doctoral. Adicionalmente, los resultados obtenidos sobre los cambios en el perfil de carbonato aportado por los cocolitos, permitieron refinar el modelo de edad en el Pacífico oriental.

El modelo de edad del testigo MD03-2616 del Atlántico occidental tropical está basado en la estratigrafía isotópica del foraminífero bentónico Uvigerina peregrina, la zonación climática de Ericson y Wollin (1956) y Kennett y Huddlestun (1972) calibrada para la cuenca de la Guayana, y en la bioestratigrafía de cocolitóforos realizada en esta Tesis Doctoral.

Los modelos de edad utilizados en este trabajo se explican detalladamente en los capítulos 5, 6 y 7 , y a manera de síntesis en el Capítulo 8.

\section{Referencias}

Andruleit, H. 1996. A filtration technique for quantitavie studies of coccoliths. Micropaleontology 42, 403406. 
Backman, J., Shackleton, N. 1983. Quantitative Biochronology of Pliocene and early Pleistocene calcareous nannofossils from the Atlantic, Indian and Pacific Ocean. Marine Micropaleontology $8,141-170$.

Baumann, K.-H. 1990. Veränderlichkeit der Coccolithophoridenflora des Europäischen Nordmeeres im Jungqartär. Berichte Sonderforschungsbereich 313, Universität zu Kiel 22, $146 \mathrm{~S}$.

Beaufort, L. 1991. Adaptation of the random settling method for quantitative studies of calcareous nannofossils. Micropaleontology 37, 415-418.

Böeckel, B., Baumann, K.-H. 2004. Distribution of coccoliths in surface sediments of the south-eastern South Atlantic Ocean: ecology, preservation and carbonate contribution. Marine Micropaleontology 51, 301-320.

Chávez, F.P., Barber, R.T. 1987. An estimate of new production in the equatorial Pacific. Deep-Sea Research Part A, 34, 1229-1243.

Dennison, J.M., Hay, W.W. 1967. Estimating the needed sampling area for subaquatic ecological studies. Journal of Paleontology 41, 706-708.

Dittert, N., Baumann, K.-H., Bickert, R., Henrich, R., Huber, R., Kinkel, H., Meggers, H. 1999. Carbonate dissolution in the deep-sea: methods, quantification and paleoceanographic application. In: Fischer, G., Wefer, G. (Eds.), Use of Proxies in Paleoceanography: Examples from the South Atlantic. Springer, Berlin, Heidelberg, pp. 255-284.

Ericson, D.B., Wollin, G. 1956. Correlation of six cores from the equatorial Atlantic and the Caribbean. Deep-Sea Res. 3, 104-125.

Flores, J.A., Sierro, F.J. 1997. Revised technique for calculation of calcareous nannofossil accumulation rates. Micropaleontology 43, 321-324.

Flores, J.A., Bárcena, M.A., Sierro, F.J. 2000. Ocean-surface and wind dynamics in the Atlantic Ocean off Northwest Africa during the last 140000 years. Palaeogeography, Palaeoclimatology, Palaeoecology 161, 459-478.

Flores, J.-A., Marino, M. 2002. Pleistocene calcareous nannofossil stratigraphy for ODP Leg 177 (Atlantic sector of the Southern Ocean). Marine Micropaleontology 45, 191-224.

Henrikson, A. 1993. A quick and easy method for estimating absolute abundances of calcareous nannofossils. INA Newsletter 15, 68.

Kennett, J.P., Huddlestun, P. 1972. Late Pleistocene Paleoclimatology, foraminiferal biostratigraphy and tephrochronology, western Gulf of Mexico. Quaternary Res. 2, 38-69.

Lisiecki, L.E., Raymo, M.E. 2005. A Pliocene-Pleistocene stack of 57 globally distributed benthic $\delta^{18} \mathrm{O}$ records. Paleoceanography 20, doi:10.1029/2004PA001071.

Mayer, L., Pisias, N., Janecek, T. et al., 1992. Explanatory notes. In L. Mayer, Pisias, N., Janacek, T. etal., Proc. ODP, Init. Repts., 138. Ocean Drilling Program. College Station TX, 13-42.

Mix, A.C., Tiedemann, R., Blum, P., et al., 2003. Proc. ODP, Init. Repts., 202. Ocean Drilling Program, College Station, TX. doi:10.2973/odp.proc.ir.202.2003.

Okada, H. 1992. Use of microbeds to estimate the absolute abundance of nannofossils. INA Newsletter 14, 96-97.

Roth, P.H., Thierstein, H. 1972. Calcareous nannoplankton: Leg 14 of the Deep Sea Drilling Project. In: Hayes, D.E., Pimm, A.C., et al., Init. Repts. DSDP 14: Washington (U.S. Govt. Printing Office), 421-485.

Rühlemann, C., Dickmann, B., Mulitza, S., Frank, M. 2001. Late Quaternary changes of western equatorial Atlantic surface circulation and Amazon lowland climate recorded in Ceara Rise deep-sea sedimentos. Paleoceanography 16, 293-305.

Samtleben, C., Schröder, A. 1992. Living coccolithophore communities in the Norwegian-Greenland Sea and their record in sediments. Marine Micropaleontology 19, 333-354.

Shipboard Scientific Party. 2003. MD132-PICASSO. IMAGES XI. Cruise Report. 76p.

Thierstein, H. R. 1980. Selective dissolution of late Cretaceous and earliest Tertiary calcareous nannofossils: Experimental evidence, Cretaceous Research 2, 165- 176.

Wei, W. 1988. A new technique for preparing quantitative nannofossil slides. Journal of Paleontology 62, 472-473.

Young, J.R., Ziveri, P. 2000. Calculation of coccolith volume and its use in calibration of carbonate flux estimates. Deep-Sea Research A 47, 1679-1700. 


\title{
5 Variations in coccolithophorid production in the Eastem Equatorial Pacific at ODP Site 1240 over the last seven glacial-interglacial cycles
}

\author{
G.-E. López-Otálvaro ${ }^{a^{*}} ;$ J.F. Flores ; F.J. Sierro ; I.Cacho
}

${ }^{a}$ Department of Geology. University of Salamanca. Plaza de la Merced s/n. E-37008 Salamanca, Spain.

${ }^{b}$ CRG Marine Geosciences, Department of Stratigraphy, Paleontology and Marine Geosciences, University of Barcelona. C/Martí i Franqués, s/n, E-08028 Barcelona, Spain.

Published in Marine Micropaleontology, "in press" doi:10.1016/j.marmicro.2007.11.009

Abstract

Key words

5.1. Introducción

5.2. Oceanographic setting

5.3. Materials and methods

5.3.1. Sediment and coccolith preparation

5.3.2. Age model and stratigraphy

5.3.3. Estimation of abundances, Nannofossil Accumulation Rate (NAR) and coccolith $\mathrm{CaCO}_{3}$ contribution

5.3.4. Taxonomic notes

5.4. Results

5.4.1. Calcareous nannofossil assemblages and the $\mathrm{N}$ ratio

5.4.2. Sedimentation and nannofossil accumulation rates

5.4.3. Coccolith carbonate mass

5.5. Discussion

5.5.1. Interval 1: MIS 15-8: $\sim 560 \mathrm{Ka}$ to $\sim 283 \mathrm{Ka}(\sim 52.10 \mathrm{mcd}$ to $\sim 29.99 \mathrm{mcd})$

5.5.2. Interval 2: MIS 8-6: $\sim 283 \mathrm{Ka}$ to $\sim 128 \mathrm{Ka}(\sim 29.99 \mathrm{mcd}$ to $\sim 14.77 \mathrm{mcd})$

5.5.3. Interval 3: MIS 5: $\sim 128 \mathrm{Ka}$ to $\sim 71 \mathrm{Ka}(\sim 14.77 \mathrm{mcd}$ to $\sim 10.82 \mathrm{mcd})$

5.5.4. Interval 4: MIS 4-1: $\sim 71 \mathrm{Ka}$ to Holocene $(\sim 10.82 \mathrm{mcd}$ to $\sim 0.03 \mathrm{mcd})$

5.6. Conclusions

Aknowledgements

References

*Correspondence to: G.-E. López-Otálvaro (gatsbyemperatriz@usal.es) 


\section{Abstract}

The calcareous nannofossil assemblage from ODP Site 1240 in the equatorial upwelling of the Eastern Pacific were analysed for the last $560 \mathrm{Ka}$. The chronological framework was set with a combination of isotopic stratigraphy, nannofossil biostratigraphy and one palaeomagnetic event.

Owing to the dominance of selected Noelaerhabdaceae and Florisphaera profunda, the $N$ index (modified from [Flores, J.A., Bárcena, M.A. y Sierro, F.J., 2000. Ocean-surface and wind dynamics in the Atlantic Ocean off northwest Africa during the last 140000 years. Palaeogeography, Palaeoclimatology, Palaeoecology 161, 459-478]) was used to investigate the fluctuations of the nutri-thermocline in the Eastern Equatorial Pacific Ocean. This ratio measures the relative proportion of taxa that usually live in the upper photic zone in relation to those that usually thrive in the lower photic zone. Temporal changes in the relative abundances of these species over the last 560 Ka may therefore be related to variability in surface water conditions. Patterns indicate by the $N$ index, were supported by the nannofossil accumulation rate and the total $\mathrm{CaCO}_{3}$ coccolith content, allowing identification of four palaeoceanographic intervals. Interval 1 (Marine Isotope Stage-MIS 14-8) is characterised by the highest index of palaeoproductivity, maximum coccolith accumulation rates and the highest carbonate content, essentially produced by Gephyrocapsa caribbeanica. These indicators imply a strengthening of the Southern Hemisphere circulation that allowed enhanced upwelling conditions, possibly related to dominant La Niña-like conditions. Interval 2 (MIS 8-6) displays frequent fluctuations of high $N$ index values, in agreement with the slight rise of $F$. profunda and the warm taxa. Both the fall in the nannofossil accumulation rate (NAR) and in the coccolith $\mathrm{CaCO}_{3}$ content were responses to the decrease in $G$. caribbeanica abundance. These events are still associated with high surface productivity related to variations in intensity of the southeast Trade winds and periodically intensified upwelling, conditions related to La Niña-like events with some fluctuations. Interval 3 (MIS 5) is characterised by atypical low $\mathrm{N}$ values, a low NAR, and a low coccolith $\mathrm{CaCO}_{3}$ content, suggesting that the Southern Hemisphere circulation was reduced and that the upwelling system was weakened, allowing us to infer that an El Niño-like event was the dominant condition. Finally, during Interval 4 (MIS 4-1), the system was restablished; the $N$ index was high again, with some inflections, and correlated with an increase in the NAR and coccolith carbonate. These indicators are related to dominant southeast Trades and equatorial currents that favoured cool and nutrient-rich waters, suggesting a La Niña-like condition with some fluctuations. 
Key words: coccolithophores, palaeoproductivity, Pleistocene, coccolith carbonate, Nannofossil Accumulation Rate (NAR), eastern equatorial Pacific, ENSO

\subsection{Introduc ción}

In this study we analysed the evolution of calcareous nannofossil assemblages over the last seven glacial-interglacial cycles to elucidate the oceanographic response of the Eastern Equatorial Pacific (EEP) to climate change. Calcareous nannofossils, a primarily algal group, are good environmental proxies owing to their temporal and spatial distribution (Brand, 1994), and are hence useful as a tool for palaeoceanographic reconstructions. Their highest relative abundances within the phytoplankton occur in warm and oligotrophic waters (Brand, 1994), but their highest absolute abundances occur in eutrophic regions, such as equatorial upwelling regions and on the outer continental shelves (Mitchell-Innes and Winter, 1987; Brand, 1994). The aims of this study are to investigate variations in the nannofossil assemblage, their contribution to carbonate production and export during the last $560 \mathrm{Ka}$ (in order to reconstruct the dynamics of the nutri-thermocline in the EEP) and the influence of the long-term El Niño-like and La Niña-like settings.

The Eastern Equatorial Pacific (EEP) is an area of high productivity associated with seasonal variations of the Trade wind system and with the interannual variability of the ENSO system (EI Niño Southern Oscillation, Philander, 1995; Clement et al., 1999; Fedorov and Philander, 2000). The major role of the EEP in the past global climate system has been documented by Chavez and Barber (1987), who stated that this region is responsible for as much as $50 \%$ of the global "new production". Long-term changes in palaeoproductivity and in the dynamics of the ocean surface currents in the EEP are driven by the Trade wind system (Philander, 1995). Furthermore, the Eastern Pacific is a key part of the El Niño-Southern Oscillation. This has two phases, the warm one (El Niño) and the cool one (La Niña), both of which cause oceanographic and atmospheric anomalies around the Earth (e.g. Philander, 1995; Fedorov and Philander, 2000). El Niño events occur in the Pacific when the differences in surface pressure across the tropical Pacific are small and consequently the Trade winds are weak (Fig. 5.1). Advection and upwelling in the Equatorial Cold Tongue (ECT) diminish because of the eastward advection of warm nutrient-depleted Western Equatorial Pacific surface waters, thickening the EEP mixed layer and markedly depressing the thermocline and nutricline (Philander, 1995; Fedorov and Philander, 
2000). The ECT in the Eastern Equatorial Pacific basin is a region associated with cool waters, forming a temperature minimum at the equator (Wyrtki, 1981), and with high-oxygen waters (Lukas, 1986). During La Niña events, the differences in surface pressure across the tropical Pacific are unusually large; the Trade winds are intense, and this strengthens advection and upwelling in the ECT, with the subsequent rise of the nutricline and thermocline and a lowering of sea surface temperatures (SST; Philander, 1995; Fedorov and Philander, 2000). During El Niño the warm pool of the Panama Basin moves southward and during La Niña the ECT is markedly enhanced and the nutricline and thermocline rise (Philander, 1995).

\subsection{Oceanographic setting}

ODP Site 1240 is situated north of Carnegie Ridge in the equatorial divergence zone, and south of the Equatorial Front (Mix et al., 2003; Fig. 5.1). This Equatorial Front is close to $30 \mathrm{~N}$ and forms the boundary between two water masses: the ECT and the warm pool of the Panama Basin. The warm pool is the warm low-salinity tropical surface water associated with the North Equatorial Counter-Current (NECC) (Wyrtki, 1981). The Cold Tongue is the cold and high-salinity nutrientrich water south of the front. This tongue comprises the South Equatorial Current (Pak and Zaneveld, 1974; Wyrtki, 1981), the Equatorial Undercurrent (EUC) and the Peru Current (PC) (Pak and Zaneveld, 1974). It is enhanced from August to October and weakened from February to March.

The PC flows northward, toward the equator, introducing cool saline, nutrient-rich waters. The PC merges with the SEC in the Galapagos region, feeding the coastal upwelling. The EUC is $100 \mathrm{~m}$ thick and flows eastward beneath the SEC, south of $3{ }^{\circ} \mathrm{N}$. It is formed of cooler and saltier waters enriched in nutrients that upwell in the equatorial divergence zone (Wyrtki, 1981) and it drives oxygen-rich waters from $150^{\circ} \mathrm{E}$ to Ecuador at the equator (Helly and Levin, 2004). Equatorial upwelling is attributed to longitudinal divergence induced by the southeast Trades and vertical mixing of the EUC (Pak and Zaneveld, 1974; Wyrtki, 1981). Lyle et al. (2002) located the equatorial high productivity zone, related to the upwelling of nutrient-rich and colder waters, between $1^{\circ} \mathrm{N}$ and $1^{\circ} \mathrm{S}$. The California Current (CC) is driven southward by the northeast Trades and feeds the easterly North Equatorial Current (NEC) with cooler and more saline waters. The NECC flows eastward and develops a cyclonic gyre when it reaches the coast of Central America, giving way to a region of upwelling and a shallower thermocline called the Costa Rica 
Dome and providing westward-flowing surface waters to the NEC (Hofmann, et al., 1981) (Fig. 5.1). The NECC flows eastward from the western equatorial "warm pool" north of $5^{\circ} \mathrm{N}$ (Wyrtki, 1981).

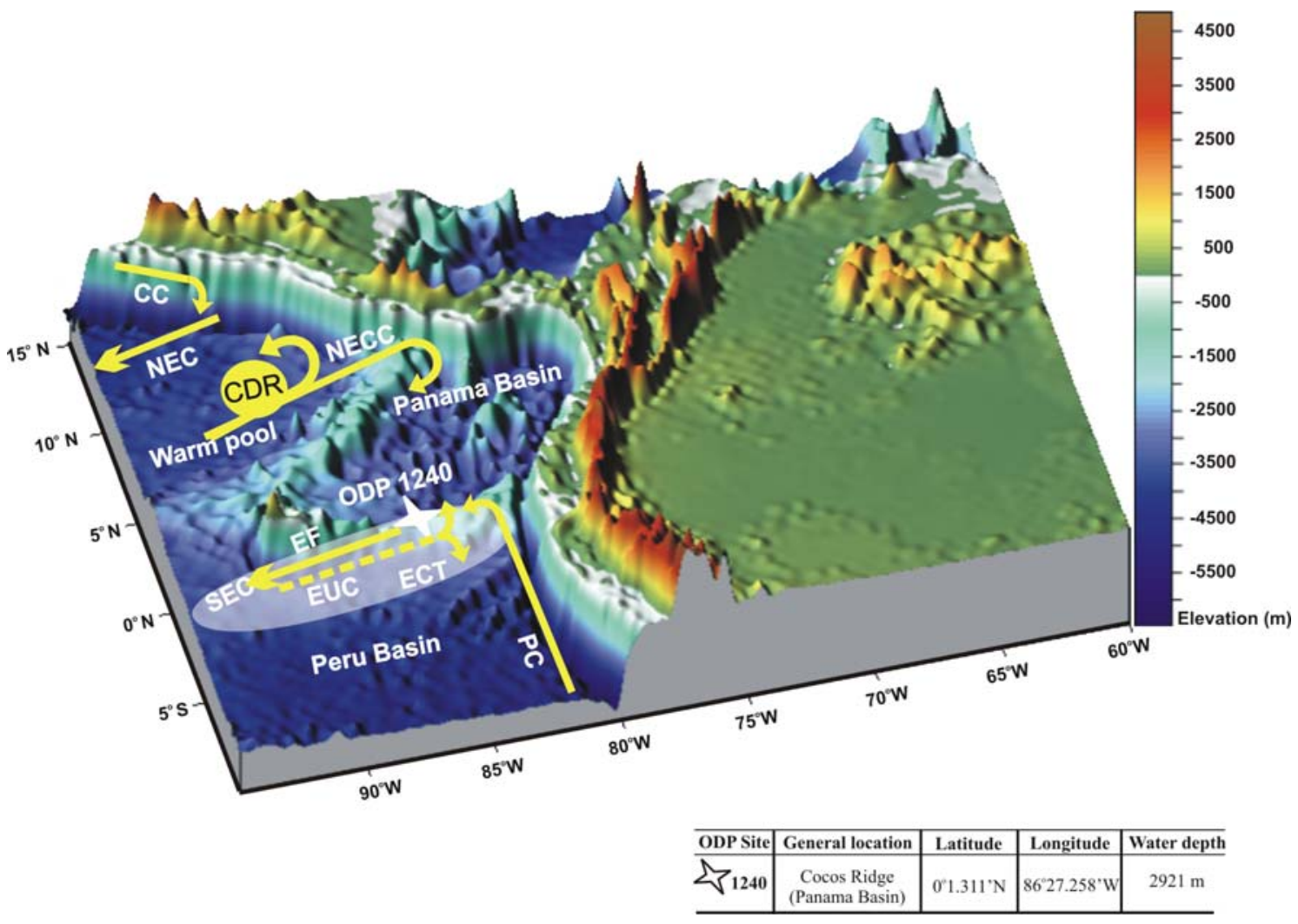

Fig. 5.1. 3-D Main surface and subsurface oceanic circulation features in the equatorial upwelling in response to atmospheric dynamics. Colour scale highlighting major bathymetric features (taken from topography database from Smith and Sandwell, 1997).Surface currents are indicated by solid lines and subsurface currents are indicated by dashed lines. PC: Peru Current, SEC: South Equatorial Current, EUC: Equatorial Undercurrent, NEC: North Equatorial Current, CC: California Current, NECC: North Equatorial Counter Current, CDR: Costa Rica Dome, ECT: Equatorial Cold Tongue (Adapted from Fiedler and Talley, 2006 and Kessler, 2006).

The Trades converge onto the ITCZ, located north of the equator in the EEP. This asymmetry above the EEP was explained by Philander et al. (1996) as being the result of ocean-atmosphere interactions and of the geometry of the American continent. The ITCZ is located over warmer SST associated with the eastward NECC. Precipitation in the ECT is lower than in the northeastern tropical region of the EEP (Philander et al., 1996; Fig. 5.1). When the southeast Trades are fully developed (August to December), the ITCZ is at its northernmost position, at about $10^{\circ} \mathrm{N}$; the SEC and the NECC are stronger, and the CC contributes waters to the NEC up to about $20 \circ$ N. From February to April, the southeast Trade winds are weakened and the northeast Trades are most intense. Thus, the ITCZ is at its southernmost position of the year, always north of the equator, causing the lowest surface salinity values and the highest sea surface temperatures (SST) of the year (Philander et al., 1996). 


\subsection{Materials and methods}

\subsubsection{Sediment and coccolith preparation}

The sediments from the upper $52.10 \mathrm{mcd}$ (meter composite depth) of ODP Site 1240 consist of a mixture of biogenic calcite and opal, dominated by nannofossils ( $40 \%$ to $\sim 80 \%)$ and diatoms ( $10 \%$ to $\sim 40 \%$ ) (Mix et al., 2003). 145 samples were prepared in order to analyze the calcareous nannofossil assemblage, using the quantitative settling technique of Flores and Sierro (1997). This allows calculation of the abundances of nannofossils per gram of sediment. $0.2 \mathrm{~g}$ of dry bulk sediment was diluted in a $10 \mathrm{ml}$ flask with buffered distilled water. Then, this mixture was briefly sonicated (20 seconds) in an ultrasonic bath. $0.1 \mathrm{ml}$ of the solution was pipetted out and homogenised on a Petri-dish with a cover slip on its bottom. This Petri-dish had previously been filled with buffered distilled water and unflavoured gelatine to ensure uniform distribution of the sediment on the slide. Then, the cover slip was mounted with Canada Balsam on a slide.

Using a polarised light microscope (1250x), more than 500 specimens per sample were counted from a variable number of viewing fields. Reworked Miocene, Pliocene and Pleistocene nannofossils were also counted, but were always rare. Additional Scanning Electron Microscope (SEM) analyses were carried out on selected samples using the SEM of the Geological Institute, Kiel. These analyses were used to settle taxonomic aspects and to observe preservation features, confirming a good to moderate preservation of the calcareous nannofossils throughout the site. (Appendices A, B, C).

\subsubsection{Age model and stratigraphy}

The age model used in this study is a combination of nine control points of the oxygen isotope record (0-329 Ka; Cacho et al., personal communication), a biostratigraphic event (LAD of $P$. lacunosa; Flores et al., 2006), and a palaeomagnetic datum at $780 \mathrm{Ka}$ (Mix et al., 2003) (Table 5.1, Fig. 5.2). The age model for the interval between 0-329 Ka was developed at the University of Barcelona, tuning the oxygen isotopic curve of the planktonic foraminifera Globigerinoides ruber (white) to the $\delta^{180} \mathrm{O}$ record of G. ruber (white) of ODP Site 677 of Shackleton et al. (1990) for the last $329 \mathrm{Ka}$ (Cacho et al., personal communication 2006). 


\begin{tabular}{cccc}
\hline Control Points & Depth (mcd) & Age (Ka) & Authors \\
\hline$\delta^{18}$ O: G. ruber white & 0.01 & 2 & Cacho et al; pers. comm. 2006 \\
\hline$\delta^{18}$ O: G. ruber white & 2.33 & 17 & Cacho et al; pers. comm. 2006 \\
\hline$\delta^{18}$ O: G. ruber white & 10.07 & 65 & Cacho et al; pers. comm. 2006 \\
\hline$\delta^{18}$ O: G. ruber white & 14.05 & 122 & Cacho et al; pers. comm. 2006 \\
\hline$\delta^{18}$ O: G. ruber white & 14.97 & 132 & Cacho et al; pers. comm. 2006 \\
\hline$\delta^{18}$ O: G. ruber white & 18.07 & 164 & Cacho et al; pers. comm. 2006 \\
\hline$\delta^{18}$ O: G. ruber white & 27.14 & 245 & Cacho et al; pers. comm. 2006 \\
\hline$\delta^{18}$ O: G. ruber white & 30.23 & 286 & Cacho et al; pers. comm. 2006 \\
\hline$\delta^{18}$ O: G. ruber white & 33.00 & 329 & Cacho et al; pers. comm. 2006 \\
\hline Biostratigraphic datum: LAD P. lacunosa & 43.66 & 458 & Flores et al., 2006 \\
\hline Paleomagnetic datum & 70.00 & 780 & (Mix et al., 2003) \\
\hline
\end{tabular}

Table 5.1. Control points used to develop the age model.

Two biostratigraphic events have been identified in ODP Site 1240: the LAD (Last Appearance Datum) of Pseudoemiliania lacunosa at $44.14 \mathrm{mcd}$ (Flores et al., 2006), and FAD (First Appearance Datum) of Emiliania huxleyi at 29.03 mcd (present work), corresponding to 458-Ka (Marine Isotope Stage-MIS 12) and 270-Ka (MIS 8). These events were dated by Thierstein et al. (1977) at $458 \mathrm{Ka}$ for the LAD of Pseudoemiliania lacunosa and $268 \mathrm{Ka}$ for the FAD of E. huxleyi; i.e., slightly above ours (Fig. 5.2).

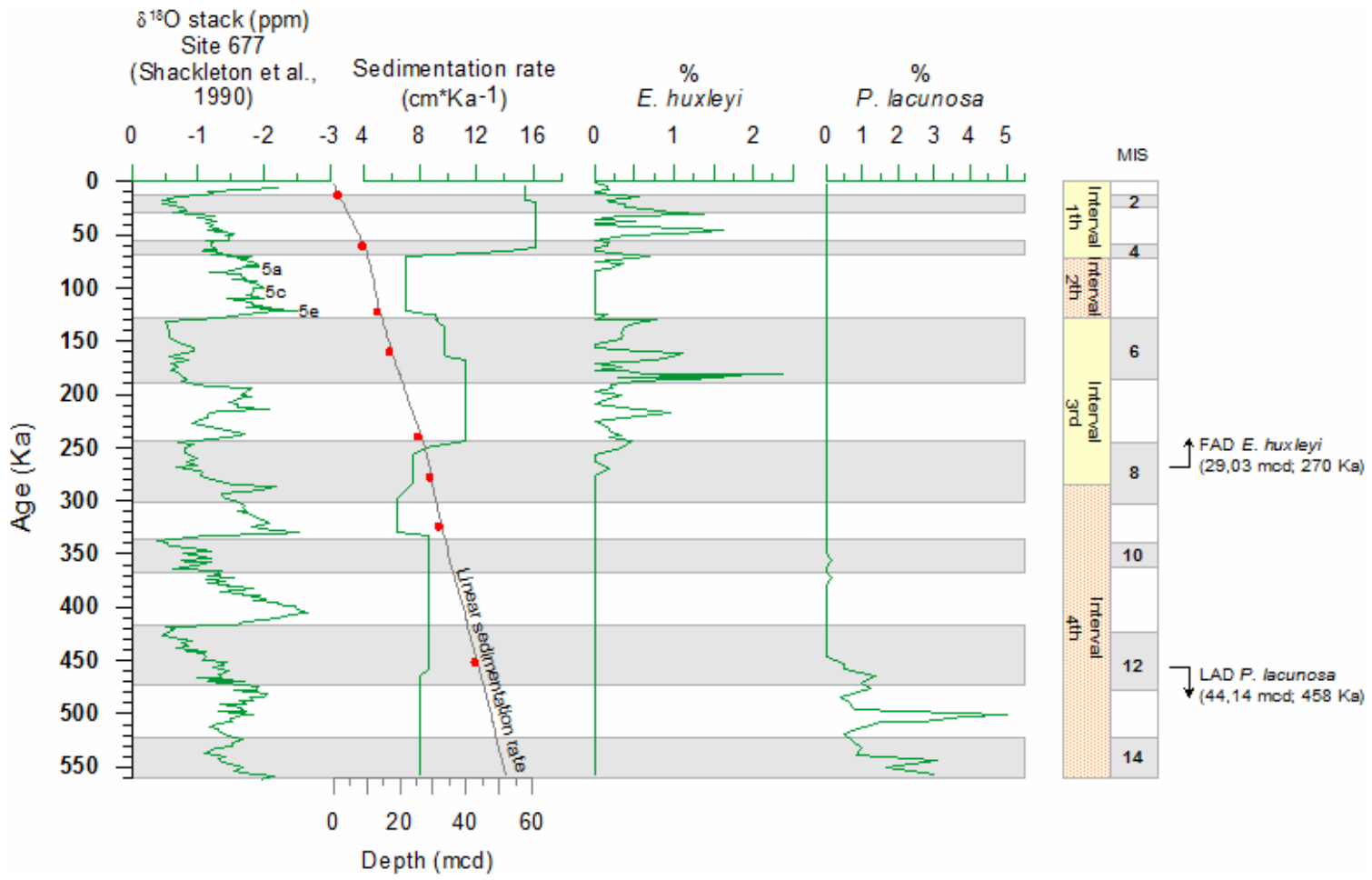

Fig. 5.2. Oxygen Isotope Stack from Site 677, compared to sedimentation rates and biostratigraphic events identified at ODP Site 1240: FAD (First Appearance Datum) of Emiliania huxleyi and LAD (Last Appearance Datum) of Pseudoemiliania lacunosa, (Table 5.2). Grey bars indicate glacial isotope stages 2 through 14. 5a, 5c, 5e: substages of MIS 5 (MIS, Marine Isotope Stage). mcd: meters composite depth. Small circles in linear sedimentation rates indicate control points. 
Unfortunately there is no available oxygen isotope record for the interval between 329 and 560 $\mathrm{Ka}$ and thus nannofossil stratigraphy and palaeomagnetism are the only tools to obtain additional datum levels of chronological significance. The nannofossil LAD of $P$. lacunosa at $458 \mathrm{Ka}$ dated by Thierstein et al. (1977) and the palaeomagnetic event dated in $780 \mathrm{Ka}$ by Mix et al. (2003) at Site 1240, were the reference points used for that period (Table 5.1). The age model was obtained by linear interpolation between these reference points using the "Analyseries" software of Paillard et al. (1996). It is possible that the age model for Site 1240 can be slightly improved in the future when the oxygen isotope stratigraphy is extended back in time.

\subsubsection{Estimation of abundances, Nannofossil Accumulation Rate (NAR) and coccolith $\mathrm{CaCO}_{3}$ contribution}

Absolute coccolith abundances (nannofossils ${ }^{*} \mathrm{~g}^{-1}$ ) and the NAR were calculated using the formula given by Flores and Sierro (1997). The NAR (nannofossils ${ }^{*} \mathrm{Cm}^{-2^{*} \mathrm{Ka}-1}$ ) was calculated using estimations of the number of coccoliths per gram (coccolith $\left.{ }^{*} \mathrm{~g}^{-1}\right)$, GRAPE densities $\left(\mathrm{d}, \mathrm{g}^{*} \mathrm{~cm}^{-3}\right)$, obtained by Mix et al. (2003), and the linear sedimentation rate (SR, $\left.\mathrm{cm}^{*} \mathrm{Ka}^{-1}\right)$, derived from the age model. GRAPE densities were used since dry sediment densities were not available.

The contribution of coccoliths to the overall carbonate flux (Mass, $\mathrm{pg}^{*} \mathrm{~g}^{-1}$ ) was assessed using the method proposed by Young and Ziveri (2000), using the species-specific mean coccolith length $\left(\mu m^{3}\right)$, absolute abundances in the sediment (nannofossils ${ }^{*} g^{-1}$ ), the shape factor $\left(k_{s}\right)$ for each species and the calcite density $\left(2.7 \mathrm{pg}^{*} \mu \mathrm{m}^{-3}\right)$. Table 5.2 summarizes the source of coccolith $k_{s}$ values, mean coccolith length, and the coccolith carbonate content for each species. This method allows the estimation of coccolith-derived $\mathrm{CaCO}_{3}$ from individual species, although a significant cumulative error (around 50\%) is involved (Young and Ziveri, 2000). A key potential source of errors is variation in size of individual species between samples. Also, broken coccoliths were not taken into account (although they do not appear to be significant), and hence the coccolith carbonate contribution could be higher than computed. So, the calculations of the coccolith carbonate content should be interpreted with caution.

The $N$ ratio palaeoproductivity proxy was proposed by Flores et al. (2000) based on the relative abundance of the main surface eutrophic species (small Noelaerhabdaceae, $R$ ) and the main lower photic zone species (Florisphaera profunda, $F$ ). Thus, high values indicate strong upwelling and shallow stratification (values closer to 1 ) while low values indicate weak upwelling and deep 


\begin{tabular}{|c|c|c|c|c|c|}
\hline Species & $K s$ & Ks source & mean length & $\begin{array}{l}\text { Vol at mean } \\
\text { length }(\mu \mathrm{m}) 3\end{array}$ & 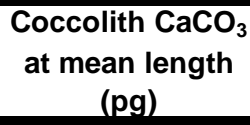 \\
\hline Calcidiscus leptoporus & 0.080 & Young and Ziveri, 2000 & 7.0 & 27.440 & 74.088 \\
\hline Emiliania huxleyi & 0.020 & Young and Ziveri, 2000 & 2.5 & 0.313 & 0.844 \\
\hline Florisphaera profunda & 0.040 & Young and Ziveri, 2000 & 3.3 & 1.437 & 3.881 \\
\hline Gephyrocapsa caribbeanica & 0.060 & $\begin{array}{l}\text { Adopted from G. oceanica's Ks } \\
\text { maximum value }\end{array}$ & 3.0 & 1.620 & 4.374 \\
\hline small Noerlabdaceae (G. ericsonii and G. aperta) & 0.050 & Adopted from G. ericsonii & 2.0 & 0.400 & 1.080 \\
\hline Gephyrocapsa muellerae & 0.050 & Young and Ziveri, 2000 & 3.0 & 1.350 & 3.645 \\
\hline Gephyrocapsa oceanica & 0.050 & Young and Ziveri, 2000 & 3.5 & 2.144 & 5.788 \\
\hline Large Gephyrocapsa & 0.050 & Adopted from G. oceanica & 4.5 & 4.556 & 12.302 \\
\hline Helicosphaera carteri & 0.050 & Young and Ziveri, 2000 & 10.0 & 50.000 & 135.000 \\
\hline Pseudoemiliania lacunosa & 0.040 & $\begin{array}{l}\text { Adopted from E. huxleyi type A } \\
\text { normally calcified }\end{array}$ & 4.5 & 3.645 & 9.842 \\
\hline \multicolumn{6}{|l|}{ Warm taxa: } \\
\hline Calciosolenia murrayi & 0.030 & Young and Ziveri, 2000 & 3.5 & 1.286 & 3.473 \\
\hline Neosphaera coccolithomorpha & 0.015 & Young and Ziveri, 2000 & 6.5 & 4.119 & 11.122 \\
\hline Oolithotus cavum & 0.070 & Adopted from O. cavum & 4.0 & 4.480 & 12.096 \\
\hline Oolithotus fragilis & 0.070 & Young and Ziveri, 2000 & 5.0 & 8.750 & 23.625 \\
\hline Pontosphaera spp. & 0.050 & $\begin{array}{l}\text { Adopted from H. carteri } \\
\text { (Zygodiscales) }\end{array}$ & 8.0 & 25.600 & 69.120 \\
\hline Rhabdosphaera clavigera & 0.025 & Young and Ziveri, 2000 & 8.0 & 12.800 & 34.560 \\
\hline Syracosphaera spp. & 0.030 & Adopted from S. pulchra & 4.0 & 1.920 & 5.184 \\
\hline Umbellosphaera spp. & 0.010 & Young and Ziveri, 2000 & 4.0 & 0.640 & 1.728 \\
\hline Umbillicosphaera sibogae foliosa & 0.060 & Young and Ziveri, 2000 & 4.0 & 3.840 & 10.368 \\
\hline Umbillicosphaera sibogae hulburtiana & 0.050 & $\begin{array}{l}\text { Adopted from U. sibogae } \\
\text { sibogae }\end{array}$ & 3.5 & 2.144 & 5.788 \\
\hline Umbillicosphaera sibogae sibogae & 0.050 & Young and Ziveri, 2000 & 4.0 & 3.200 & 8.640 \\
\hline
\end{tabular}

Table 5.2. Summary of coccolith shape constants used (Ks) and carbonate coccolith calculations for each species (mean length) at Site 1240. 
stratification (values closer to 0). In this work, this function was modified to include Gephyrocapsa ocecanica $(G 0)$, a species that was absent from the original function. G. oceanica is one of the most abundant surface species in the core and has mainly been reported in surface waters in tropical and subtropical areas (Okada and Honjo, 1973; Okada and Mclntyre, 1979; Beaufort and Buchet, 2003), in upwellings areas of low latitudes: the Panama Basin (Giraudeau et al., 1993; Hagino and Okada, 2004; Martínez et al., 2005), northwest Africa (Geitzenauer et al., 1977), the Red Sea (Winter, 1982) and monsoon-induced upwelling waters (Houghton and Guptha, 1991), and it has also been described in the relatively warm upwelling waters of the Beguela area (Giraudeau, 1992). Accordingly, the modified $N$ ratio proposed and used in this core is:

$$
N^{\prime}=\frac{R+G o}{R+G o+F}
$$

\subsubsection{Taxonomic notes}

The most abundant group of taxa at the site belong to the family Noelaerhabdaceae, including Gephyrocapsa oceanica, G. muellerae, G. caribbeanica, Emiliania huxleyi, small Reticulofenestra and Pseudoemiliania lacunosa. The morphotaxonomy used for the genus Gephyrocapsa was adopted from Flores et al. (2000) and is summarised in the Appendix B and Appendix of chapter 8). Small placoliths $<3 \mu \mathrm{m}$ long with an open central area and small placoliths $<2.5 \mu \mathrm{m}$ long with a closed central area were grouped as small Noelaerhabdaceae, this includes small Gephyrocapsa (G. aperta and G. ericsonii), small Reticulofenestra, and rare Emiliania huxleyi. Other small placoliths between 2.5-3 $\mu \mathrm{m}$ but with a closed central area were included in the small Noelaerhabdaceae as a small morphotype of $G$. caribbeanica. This difference in the small Noelaerhabdaceae was considered for coccolith carbonate calculations. Different sizes of $G$. oceanica were also considered for carbonate calculations (medium Gephyrocapsa 3-4 $\mu \mathrm{m}$ and large Gephyrocapsa 4-5 $\mu \mathrm{m}$ ), but later these sizes were lumped as G. oceanica for palaeoecological purposes. Calcidiscus leptoporus and Helicosphaera carteri are subordinate species and were present throughout the record. Although Coccolithus pelagicus, a subordinate species, was very rare and sporadic, it was considered for coccolith carbonate estimations. The group of species categorised as warm and oligotrophic taxa include Umbilicosphaera sibogae, Calciosolenia murrayi, Neosphaera coccolithomorpha, Oolithotus spp., Pontosphaera spp., Rhabdosphaera clavigera, Syracosphaera spp. and Umbellosphaera spp. (Böeckel and Baumann, 2004) and were lumped together. Umbilicosphaera, a major component of the warm taxa, and includes in three species: U. sibogae, U. foliosa and U. hulburtiana. These species were counted separately but were grouped together because they did not show a 
palaeoecologically different behaviour. Also, species of the genera Oolithotus, Pontosphaera, Syracosphaera and Umbellosphaera were not distinguished by light microscopy and were counted together in their corresponding genus.

\subsection{Results}

\subsubsection{Calcareous nannofossil assemblages and the $\mathbf{N}$ ratio}

Small Noelaerhabdaceae, G. oceanica and F. profunda were the most abundant species throughout the interval studied, although F. profunda was only abundant during MIS 5 (Fig. 5.4a, 5.4b; Appendices A, B, C). Small Noelaerhabdaceae constitute between $\sim 50 \%$ and $\sim 11 \%$ of the total assemblage. In some intervals, the small Noelaerhabdaceae appeared alternately with $G$. oceanica. The relative abundances of $G$. oceanica increased up-core reaching maximum abundances of $60 \%$. F. profunda abundances increased slightly from MIS 8 (less than 10\%) to MIS 6 and then abruptly in MIS $5 \mathrm{e}$, when this species reached $\sim 62 \%$. Major changes in the composition and absolute abundance of the assemblage, the NAR and coccolith-derived $\mathrm{CaCO}_{3}$ were observed during the mid-Brunhes event between MIS 14-8 and during the warm MIS 5 (Fig. 5.3).

Another significant species is the heavily calcified G. caribbeanica, which was very abundant (up to $65 \%$ ) during the mid-Brunhes event (MIS 14-8), and sometimes also alternated in abundance with the small Noelaerhabdaceae. The highest concentration of nannofossils is observed during MIS 14 to the early phase of MIS 8, coinciding with the maximum abundances of $G$. caribbeanica and its small morphotype. A marked reduction in the absolute abundances of coccoliths from the middle of MIS 8 to the Holocene was coincided with more prominent fluctuations of $F$. profunda.

Calcidiscus leptoporus, $H$. carteri and the warm taxa, have combined abundances below $10 \%$, throughout the studied interval. However, their contribution is significant in terms of carbonate production because they produced large, heavily calcified coccoliths. H. carteri and C. leptoporus reached their maximum abundances during MIS 6 and MIS 3. G. muellerae and the warm taxa, although subsidiary in the coccolithophore assemblage, displayed opposite short-term behaviour, but fairly similar long-term patterns. Thus, both G. muellerae and the warm taxa show increases in abundance during MIS 6 and especially MIS 4-1, but within these intervals they show anticovariant patterns of peaks and troughs in abundance (Fig. 5.4a). Other species were recorded in 
very low abundances. In regard to coccolith preservation, Light and Scanning Electron Microscope observations revealed good to moderate preservation along the whole sequence studied.

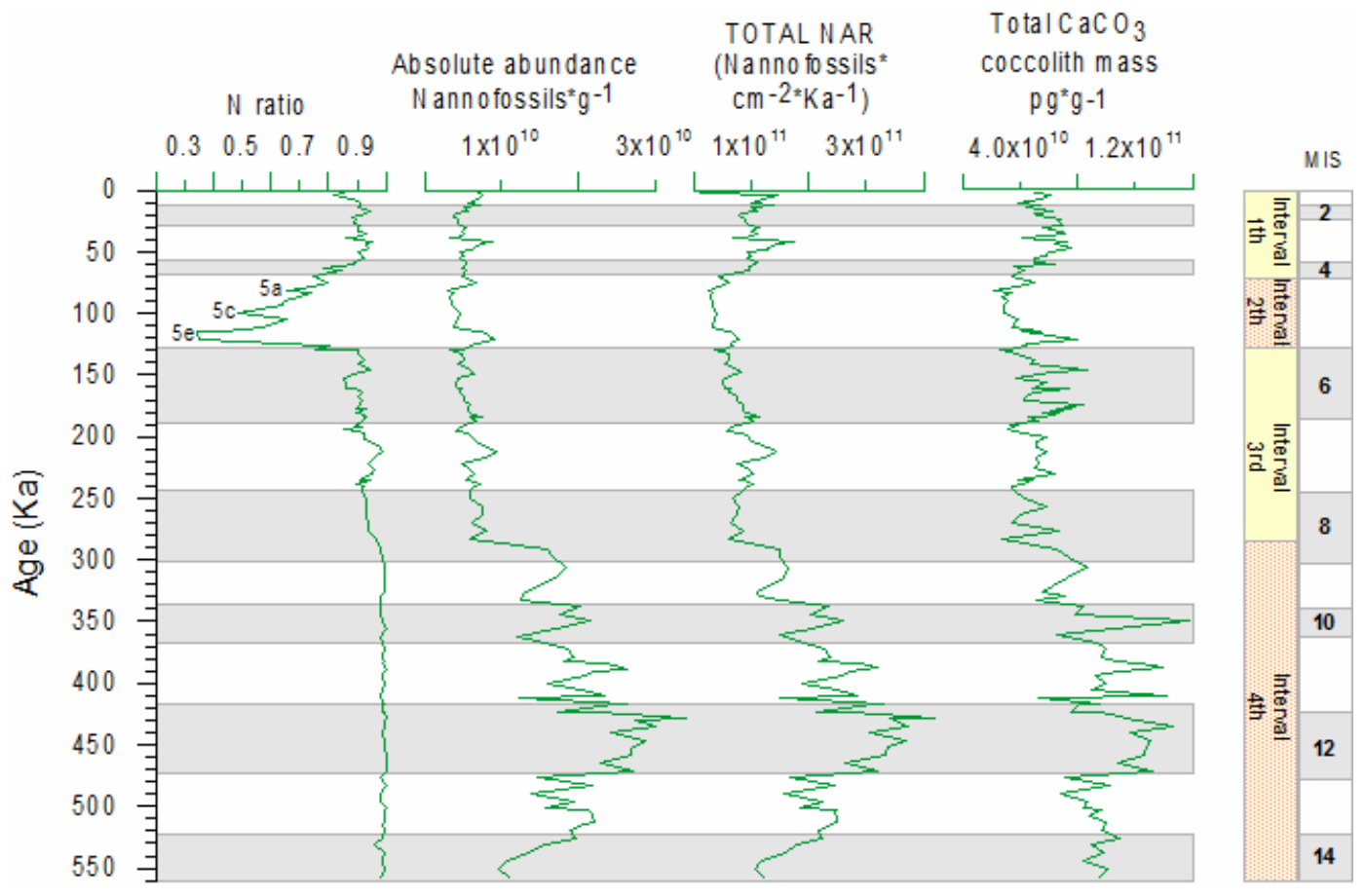

Fig. 5.3. Review of the major bioproductivity proxies at Site 1240: $N$ ratio (proportion of $F$. profunda to small Noelaerhabdaceae and G. oceanica) vs. total absolute abundance of coccoliths, their accumulation rate (NAR) and the calculated total mass of coccolith $\mathrm{CaCO}_{3}$ per gram of sediment (NB $1 \mathrm{~g}=10^{12} \mathrm{pg}$ ). Grey bars indicate glacial isotope stages 1 through 14 . 5a, 5c, 5e: substages of MIS 5 . The paleoceanographic intervals discussed in section 5 are included. MIS: Marine Isotope Stage.

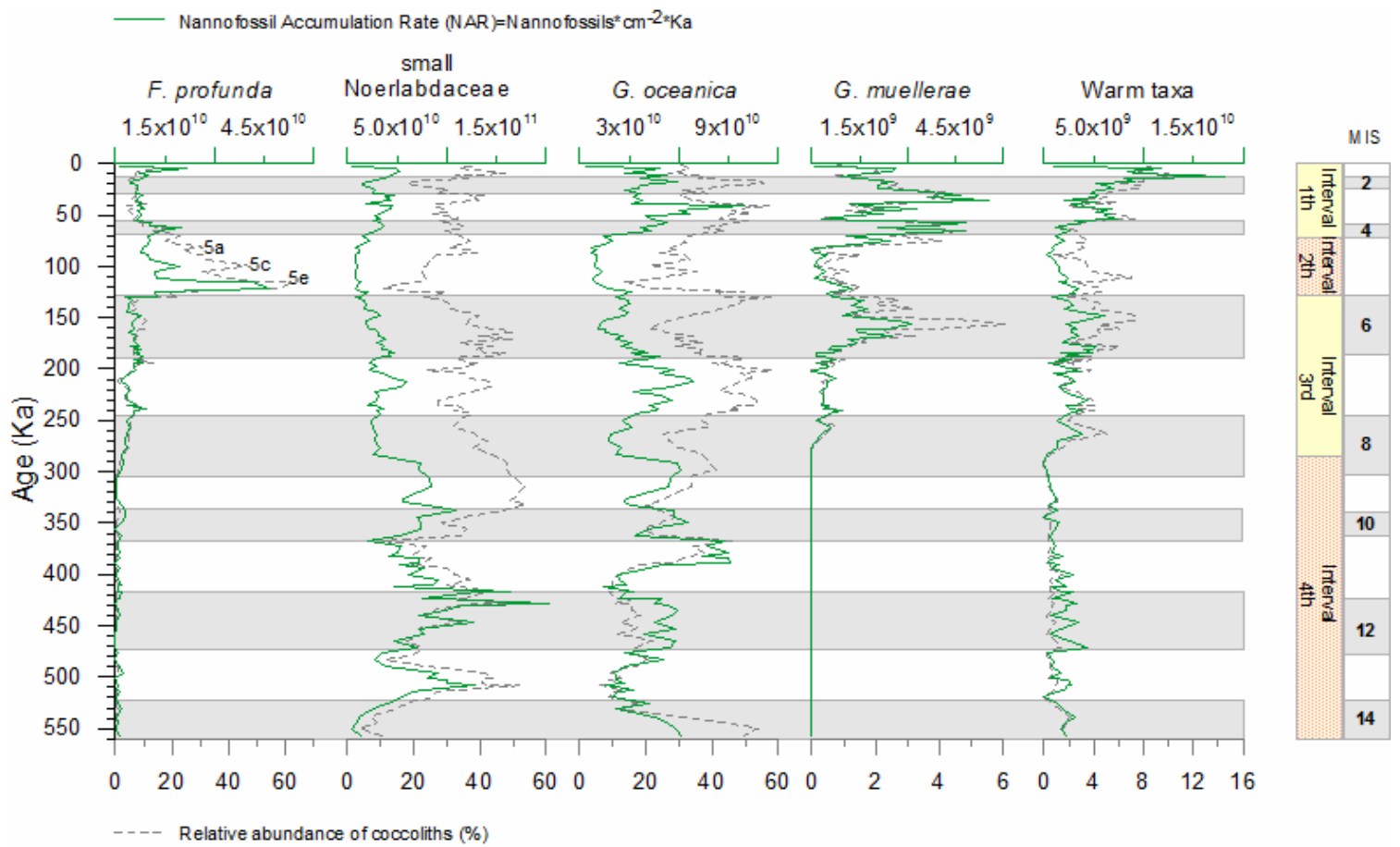

Fig. 5.4a. Relative abundances (\%) and accumulation rates of the most significant nannofossil species. Grey bars indicate glacial isotope stages 2 through 14 . 5a, 5c, 5e: substages of MIS 5 . The paleoceanographic intervals discussed in section 5 are included. 


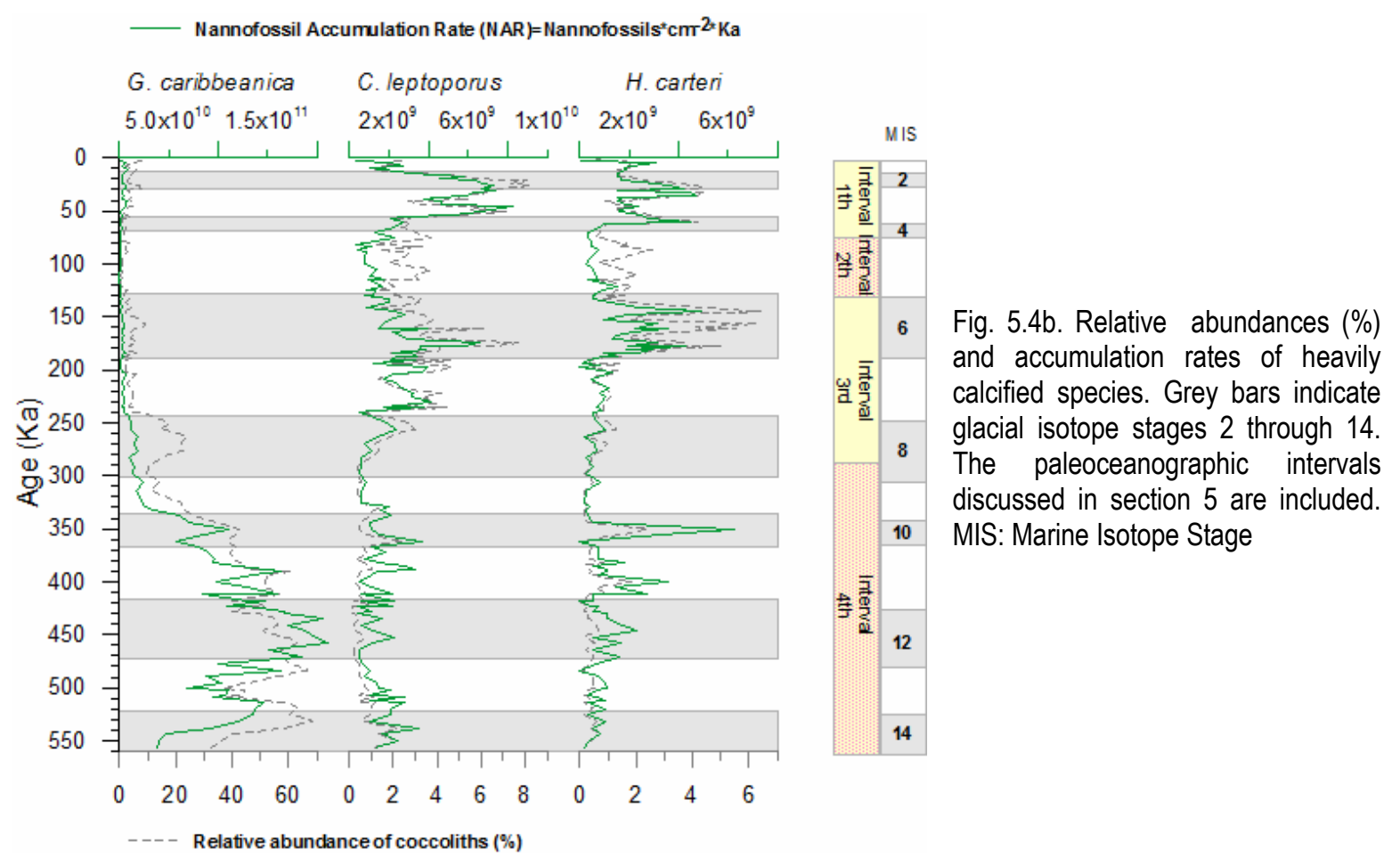

The $\mathrm{N}$ ratio (Fig. 5.3) exhibits important variations in the interval studied. The highest and most uniform N ratio values were observed from MIS 14 to the beginning of MIS 8, coinciding with the maximum fluxes of coccoliths and their maximum carbonate production. This index gradually starts to decrease during MIS 8, showing fluctuations corresponding to peaks of $F$. profunda. The $\mathrm{N}$ values fall dramatically in isotope substage $5 \mathrm{e}$, followed by an increase to pre-5e levels, which is interrupted by negative excursions in the substages $5 c$ to $5 a$. Relatively high values are reached from MIS 4 to the Holocene. A general decreasing trend in the N values over the last 560 $\mathrm{Ka}$ is evident and does not seem to be correlated with the glacial/interglacial pattern of variation in this system.

\subsubsection{Sedimentation and nannofossil accumulation rates}

The site is characterised by high average sedimentation rates $\left(9.3 \mathrm{~cm}^{*} \mathrm{Ka}^{-1}\right)$ (Fig. 5.2), maximum values being observed during MIS 4-1. By contrast, the lowest sedimentation rates occur during MIS 9-8 and MIS $5\left(\sim 7 \mathrm{~cm}^{*} \mathrm{Ka}^{-1}\right)$. The low sedimentation rates during MIS 14-10 may be an artefact of the few reference points used in the age model for this interval (Fig. 5.2). 
The total accumulation rates of calcareous nannofossils at Site 1240 are high over the last 560 Ka (Fig. 5.3), around $5 \times 10^{10}$ to $4 \times 10^{11}$ nannofossils ${ }^{*} \mathrm{~cm}^{-2 *} \mathrm{Ka}^{-1}$, and decrease upwards. Our study reveals that the major contributors to the nannofossil accumulation rates (NAR) were $G$. caribbeanica, the small Noelaerhabdaceae and G. oceanica (Figs. 5.4a and 5.4b), and their fluxes alternated reciprocally. A lower NAR coincides with the fall in the abundances of $G$. caribbeanica. The lowest nannofossil accumulation rates occurred during MIS 5, coinciding with the dominance and maximum concentration of $F$. profunda in the assemblage. In spite of the high accumulation rates of the subordinate and highly calcified species $C$. leptoporus, $H$. carteri and $U$. sibogae during MIS 6, the total NAR was low during that time.

\subsubsection{Coccolith carbonate mass}

The contribution of coccolith-derived $\mathrm{CaCO}_{3}$ varies considerably and followed a prominent pattern along the mid-Brunhes event, coinciding with the G. caribbeanica extra component of the assemblage (Fig. 5.3 and 5.4b). The highest values are found during MIS 14-8 (Fig. 5.3, 5.5a and $5.5 b)$. In spite of their low abundances, the major contributors to the coccolith carbonate are the heavily calcified species G. caribbeanica (up to $\sim 65 \%$ ), $H$. carteri (up to $\sim 60 \%$ ) and C. leptoporus (up to $\sim 50 \%$ ). Other very important calcite producers are G. oceanica, with a relative production of up to $\sim 50 \%$, and the small Noelaerhabdaceae (up to $\sim 16 \%$ ) owing to their high abundances in the assemblage (Fig. 5.5a and 5.5b). The carbonate contribution during MIS $5 \mathrm{e}$ is dominated by F. profunda.

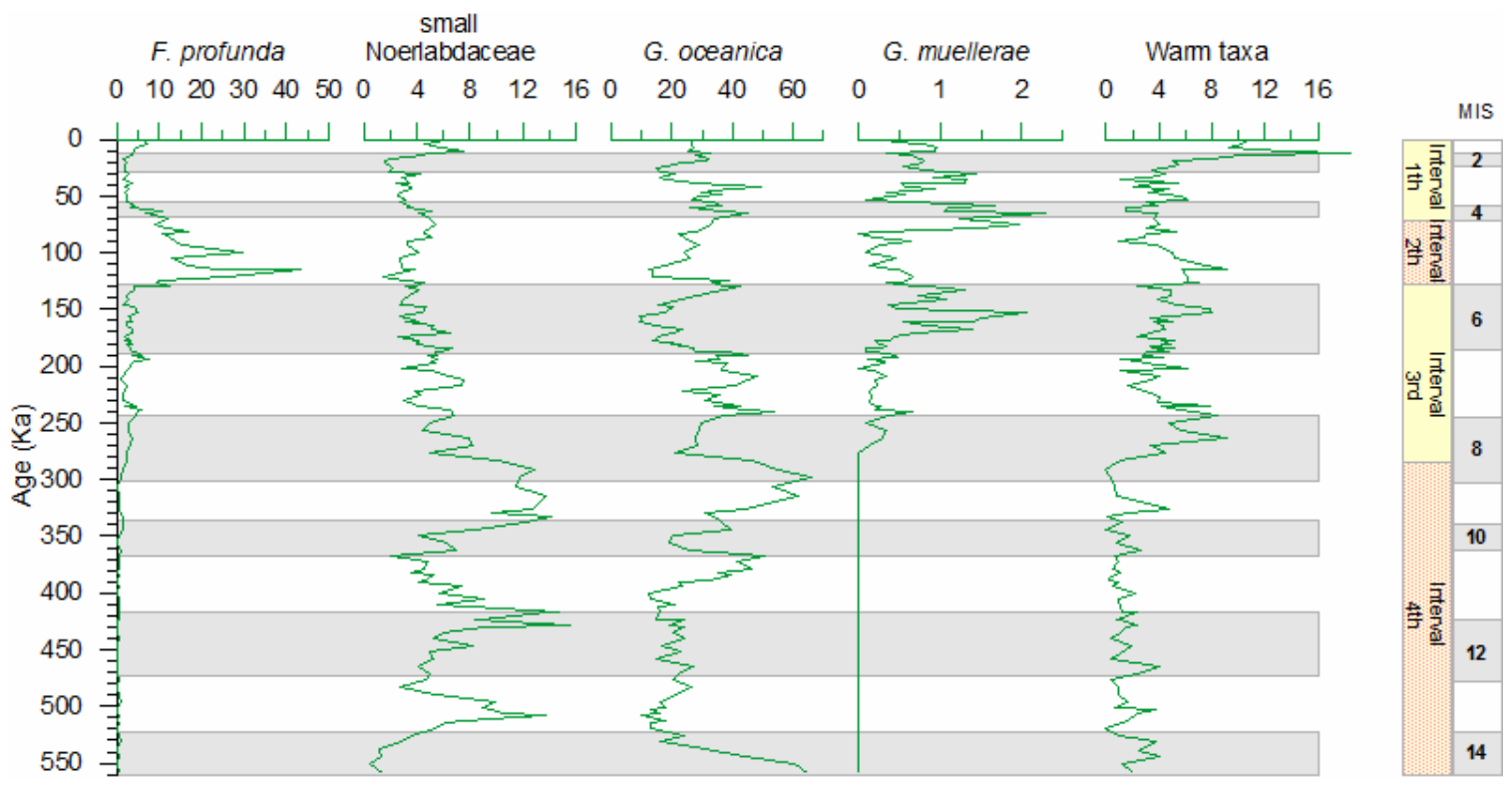

Fig. 5.5a. Coccolith carbonate contributed by the most abundant coccolithophores at Site 1240. Grey bars indicate glacial isotope stages 2 through 14. The palaeoceanographic intervals discussed in section 5 are included. MIS: Marine Isotope Stage. 


\subsection{Disc ussion}

Coccolithophores strongly contributed to the palaeoproductivity and to the accumulation of the sediments along the last $560 \mathrm{Ka}$ at ODP Site 1240. Many authors have used nannofossils, especially upper photic zone dwellers such as small Noelaerhabdaceae, (Young, 1994; Okada and Wells, 1997; Wells and Okada, 1997; Flores et al., 1999; 2000, 2003; Bollman et al., 1988; Beaufort et al., 1999; Beaufort and Buchet, 2003) and G. oceanica (see section 3.3), as palaeoproductivity proxies in upwelling areas (Young, 1994; Baumann and Freitag, 2004) and in equatorial high-productivity locations (Hagelberg et al., 1995; Weber, 1998). F. profunda normally grows at a depth close to the nutri-thermocline when the water column is stratified (Okada and Honjo, 1973; Molfino and Mclntyre, 1990a,b; Young, 1994; Okada and Wells, 1997; Wells and Okada, 1997; Beaufort et al., 1997, 1999, 2001, 2003; Beaufort and Buchet, 2003; Flores et al., 2000; Liu and Herbert, 2004; Baumann and Freitag, 2004). This species has also been considered as a sea water transparency indicator (Ahagon et al., 1993).

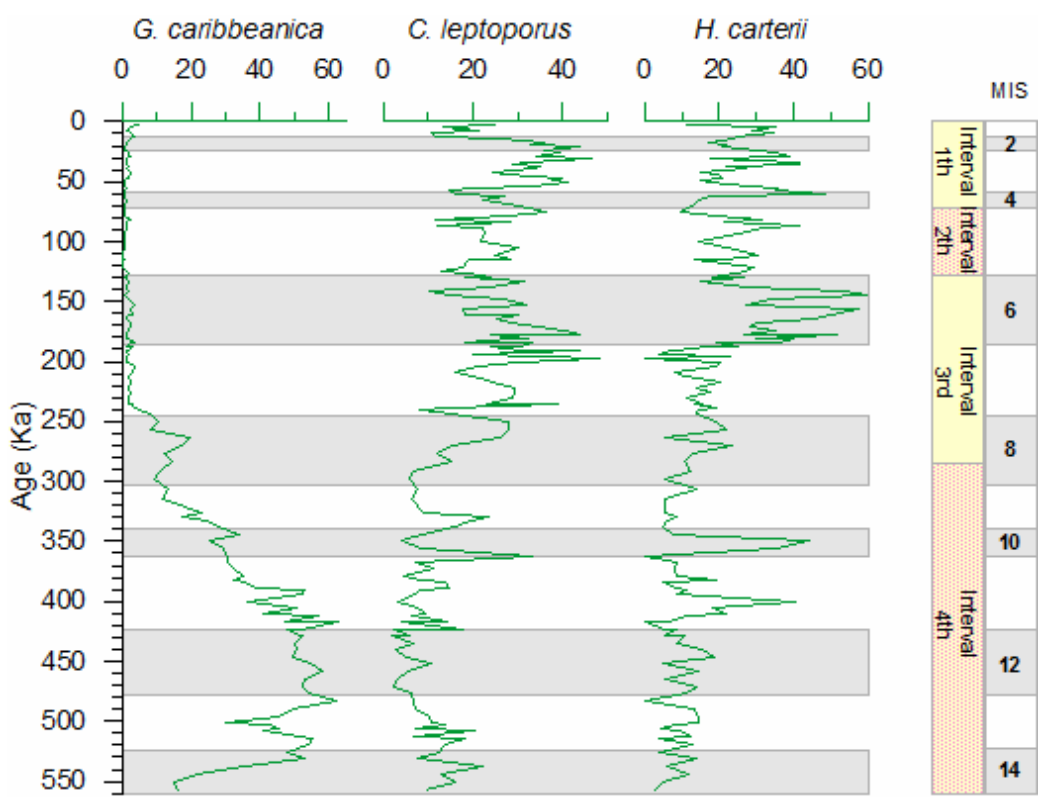

Fig. 5.5b. \% coccolith carbonate contributed by heavily calcified coccolithophores at Site 1240. Grey bars indicate interglacial isotope stages 2 through 14 . The paleoceanographic intervals discussed in section 5 are included. MIS: Marine Isotope Stage

Variations in the assemblage over the last $560 \mathrm{Ka}$ are related to changes in the dynamics of the water-column that regulate nutrient content and sea surface temperature: hence the dominance of the nannofossil species near the equator. An ecological alternation could explain the alternating dominance of small Noelaerhabdaceae (related to maximum eutrophication) and $G$. oceanica (associated with highly eutrophic and moderately warm sea surface waters) in response to slight sea surface temperature changes, as proposed by Hagino and Okada (2004) for the Equatorial Pacific. 
It is possible that the low abundance of $C$. pelagicus and $E$. huxleyi at this site was mainly temperature-induced. C. pelagicus has been reported as an inhabitant of low-temperature upwelling waters (Cachão and Moita, 2000). Okada and Honjo (1975), Houghton and Guptha (1991) linked the scarcity of E. huxleyi to the higher concentration of G. oceanica in the nutrientrich waters of the open tropical and subtropical regions of the Red Sea and the Western Pacific.

The variability of the NAR and coccolith $\mathrm{CaCO}_{3}$ contents were used here as proxies for bioproductivity, the calcified nannofossils being the main suppliers of calcium carbonate to the sediments. Coccoliths are considered to be the main component of the sediment during the interval studied (Mix, et al., 2003) and hence should be the major contributor to total carbonate for the last $560 \mathrm{Ka}$. Unfortunately, carbonate analyses are not available and it is therefore not possible to assess the contribution of coccoliths to the total carbonate in the sediments. Assemblage fluctuations provide an important source of information about palaeoproductivity and carbonate coccolith data.

Based on this analysis, a continuous record of coccolithophore palaeoproductivity was elaborated using the $\mathrm{N}$ function. Significant changes in this function allowed us to distinguish four palaeoceanographic intervals over the last 560 ka (Fig. 5.3):

\subsubsection{Interval 1: MIS 14-8: $\sim 560 \mathrm{Ka}$ to $\sim 283 \mathrm{Ka}(\sim 52.10 \mathrm{mcd}$ to $\sim 29.99 \mathrm{mcd}$ )}

This interval, characterised by coccolith-rich material has very high $N$ values, the consequence of high fertility in surface waters, which was probably associated with strong upwelling and high bioproduction. The abundance of the small Noelaerhabdaceae and G. oceanica would correspond to a well defined ECT in the EEP, caused by a shallow nutri-thermocline through the reinforcement of the EUC and the SEC, and the displacement of the ITCZ at its northernmost position due to the dominance of the southeast Trades, which favoured the proliferation of these species. The highest abundances of the upper photic zone inhabitants together with the highest NAR and the maximum concentration of coccolith carbonate (Fig. 5.4a) suggest intense upwelling episodes. These conditions were probably related to a period of higher frequency and more intense La Niña-like events (Fig. 5.6). 

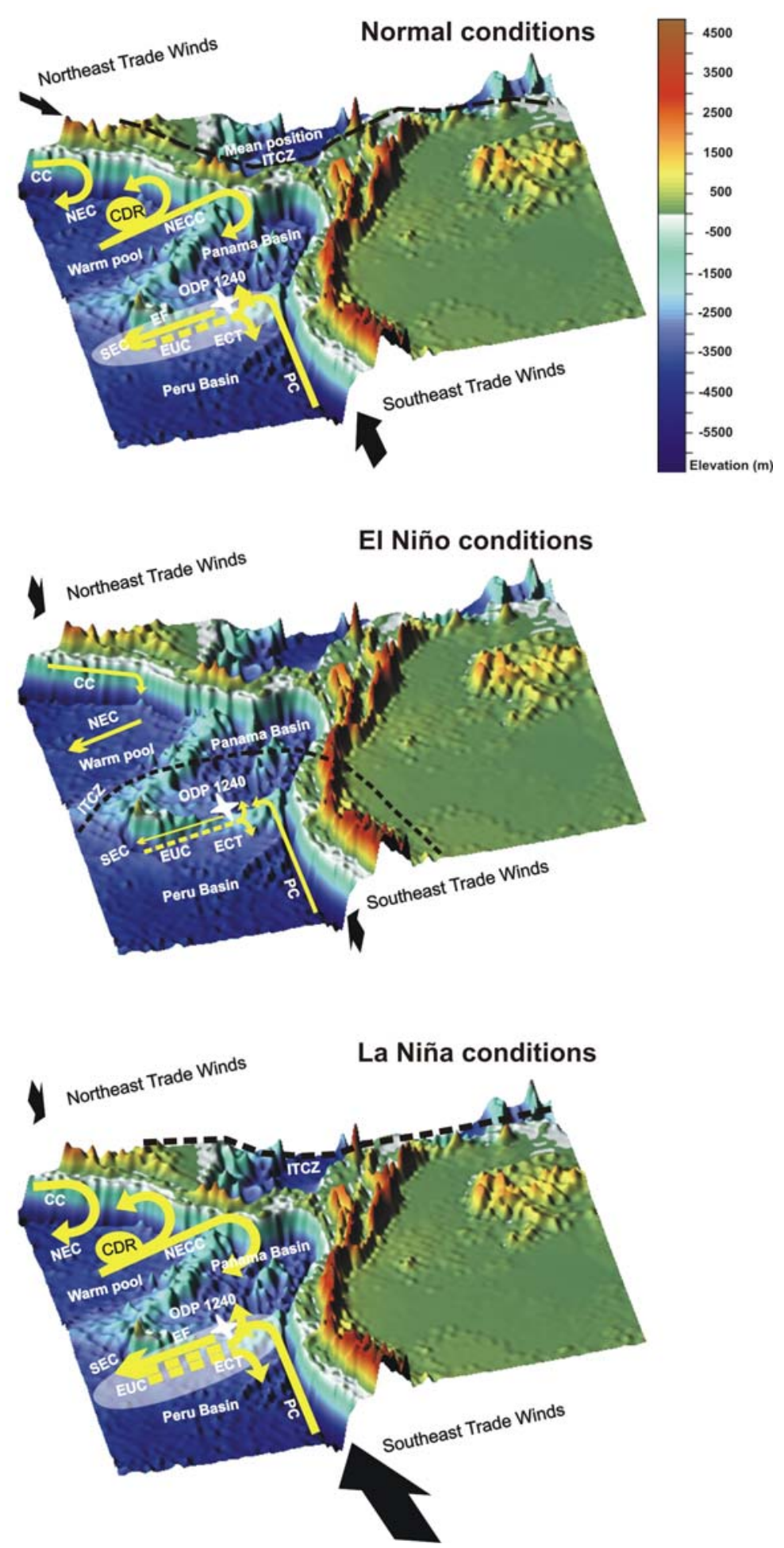

Fig. 5.6. Atmospheric and oceanic conditions during periods of normal, El Niño-like and La Niña-like conditions. PC: Peru Current, SEC: South Equatorial Current, EUC: Equatorial Undercurrent, NEC: North Equatorial Current, CC: California Current, NECC: North Equatorial Counter Current, CDR: Costa Rica Dome, ECT: Equatorial Cold Tongue, (Normal conditions adapted from Fiedler and Talley, 2006; and Kessler, 2006). ITCZ: Intertropical Convergence Zone (Mean position of the ITCZ adapted from Philander, 1995). Colour scale highlighting major bathymetric features (taken from topography database from Smith and Sandwell, 1997). 
The co-occurrence of low magnetic susceptibility, high lightness values $\left(\mathrm{L}^{*}\right)$, and high $\mathrm{CaCO}_{3}$ accumulation rates reported by Mix, et al. (2003), supports the above interpretation. Jansen et al. (1986) reported the marked northward displacement of oceanic fronts, allowing the strengthening of the Southern Hemisphere circulation in all oceans between 300-400 Ka. Other studies have searched for a connection between primary productivity, the movement of the thermo-nutricline, wind stress and ENSO events in the equatorial Indopacific oceans (Beaufort et al., 1997; 2001).

The period from MIS 14-8 has been referred to as the mid-Brunhes event and corresponds to a global increase in the carbonate content of pelagic sediments (Droxler et al., 2003) and a significant increase in the global abundance of the heavily calcified G. caribbeanica (Bollmann et al., 1998, in the Atlantic, Southwestern Pacific and Southern oceans; and by Flores et al., 1999, 2003, Baumann and Freitag, 2004, and Baumann et al., 2004, at high latitudes). G. caribbeanica was excluded from the $\mathrm{N}$ ratio because it is almost absent in the upper intervals. Neverthless, this upper photic species could be considered a proxy of high palaeoproductivity. Thus, a superimposed global or evolutionary pattern can be ruled out.

Although carbonate dissolution was intensive during the mid-Brunhes event, good to moderate coccolithophore preservation is found for this period at site 1240. This suggests that there was no vertical change in the water masses overlying the sediment, and hence the carbonate compensation depth (CCD) and the lysocline must have been deeper than $2900 \mathrm{~m}$ during even that event. The presence of a deep lysocline during this interval, when other areas of the Equatorial Pacific were subject to strong dissolution, could be the consequence of high local rates of carbonate supply to deep waters (Archer 1991a, b).

\subsubsection{Interval 2: MIS 10 to 6: $\sim 283$ to $\sim 128$ Ka ( 29.99 mcd to $14.77 \mathrm{mcd}$ )}

A slight long-term reduction in the $\mathrm{N}$ ratio starting $283 \mathrm{Ka}$ ago suggests less intense upwelling conditions in surface waters. Likewise, the progressive increase in the warm-water taxa during the last $283 \mathrm{Ka}$ seems to indicate that SSTs were probably due to variations in the depth of the nutri-thermocline. These records, together with the decreasing upward trends of the NAR and coccolith carbonate mass, were probably the result of a progressive reduction in Trade wind intensity. This is fairly consistent with the results obtained by Scharmm (1985) and Emeis et al (1995), who suggested that the Southern Hemisphere circulation was reduced over the last 500 
$\mathrm{Ka}$, favouring the warming of surface waters in the EEP. At Site 1240, this warming is more apparent during the last $283 \mathrm{Ka}$.

The oceanographic situation during this interval may have been dominated by La Niña-like conditions, punctuated by short periods in which El Niño-like events were more frequent (Fig. 5.6). This interval could have been the prelude to an oceanographic scenario that developed fully during MIS 5, with similar oceanographic contidions to those existing today during El Niño events.

\subsubsection{Interval 3: MIS 5: $\sim 128 \mathrm{Ka}$ to $\sim 71 \mathrm{Ka} \mathrm{(} 14.77 \mathrm{mcd}$ to -10.82 $\mathrm{mcd}$ )}

This interval, defined by an abrupt increase in F. profunda and so a decrease in $N$ values (Fig. 5.3, $4 \mathrm{a}$ and $4 \mathrm{~b}$ ), is characterised by a remarkable change in the coccolith assemblage, suggesting that the water column of the EEP became more stratified, leading to a reduction in nutrient supply in surface waters and a deepening of the nutri-thermocline. All palaeoproductivity proxies ( $N$ ratio, NAR, and coccolith carbonate) seem to indicate a collapse in the upwelling system (Fig. 5.3). The sediments may have been slightly affected by dissolution, resulting in a weak relative increase in F. profunda, although the presence of small Noelaerhabdaceae, the low abundances of $G$. oceanica and the identification of all coccoliths at species level suggest that dissolution, if it occurred, would have been moderate.

Evidence from other proxies, such as high magnetic susceptibility values (Mix et al., 2003), suggests reduced atmospheric circulation in the EEP, since low wind intensities favour the settling of the finest eolian magnetic particles (Hovan, 1995). A low $\mathrm{CaCO}_{3}$ content for the last interglacial period (MIS 5) has been reported previously by Lyle et al. (1988), Lyle et al. (2002), Thomas et al. (2000), Snoeckx and Rea (1994) and Murray et al. (1995) for the Eastern Tropical Pacific, suggesting low carbonate sedimentation due to low carbonate productivity at that time (Hagelberg, et al., 1995). Other proxies, such as planktonic foraminifer $\mathrm{Mg} / \mathrm{Ca}$ values, indicate the presence of very warm SSTs during MIS 5e (Lea et al. 2006), in agreement with the low $\mathrm{N}$ values and the strongly stratified water column found in this work. Beaufort and Buchet (2003) reported a relationship between the increases in F. profunda and the El Niño-like warm pool in the Western Pacific. Warmer conditions during El Niño-like events were linked to lower primary productivity during MIS 5 in the Indian and Pacific equatorial oceans (Beaufort et al., 1997; 2001). 
The above evidence suggests a deepening of the nutri-thermocline and the arrival of the warm pool at Site 1240, in response to a weakening of the southeast Trades during MIS 5e (Fig. 5.6), mostly associated with El Niño-like conditions with some fluctuations.

\subsubsection{Interval 4: MIS 4 to 1: $71-K a$ to Holocene ( 10.82 mcd to $-0.03 \mathrm{mcd}$ )}

The $\mathrm{N}$ values (Fig. 5.3a) during this interval as well as the accumulation rate of coccoliths and coccolith-derived carbonate were higher during MIS 4-1 than during the anomalous MIS 5. This situation is typically related to intense eutrophic conditions, also associated with enhanced biogenic opal production. The dominance of the southeast Trades, together with a northward displacement of the ITCZ, would have driven the ECT to the equator, resulting in high nutrient supply to the surface waters (Fig. 5.6). Atmospheric and oceanographic conditions were comparable to those observed under the influence of La Niña-like events (Fig. 5.6). The $\mathrm{N}$ ratio seems to have reversed during the Holocene, indicating a slight decline in palaeoproductivity, but it is still very high and therefore consistent with the results reported by Ravelo and Shackleton (1995), Farrell et al. (1995) and Rickaby and Halloran (2005), who suggested a shallower thermocline during the Holocene. Moreover, the increase in the frequency of fluctuations in the $\mathrm{N}$ ratio suggests that El Niño-like events were more frequent.

\subsection{Conclusions}

Our results suggest that the long-term variation in the nannofossil record for Site 1240 is an important tool for investigating the variability of the EEP and its response to palaeoceanographic and palaeoatmospheric conditions over the last seven glacial/interglacial cycles. In particular, the nannofossil assemblage, the nannofossil accumulation rate, and coccolith-derived $\mathrm{CaCO}_{3}$ provide detailed historical information about surface water palaeoproductivity and the displacement of the nutri-thermocline, both due to variations in intensity of the Trade wind system.

The dominance of small Noelaerhabdaceae, G. oceanica and G. caribbeanica indicates that the presence of nutrient-rich waters is indeed characteristic of high productivity in the upper photic zone, due to a shallow nutri-thermocline. These species imply the influence of the cool and subpolar waters and stronger Trade winds. The dominance of $F$. profunda is linked to a reduction in the intensity of the southeast Trade winds, a weakening of the upwelling, and the arrival of the 
warm pool at Site 1240. Likewise, small Noelaerhabdaceae and G. oceanica show an ecological alternance between high nutrient availability and cool temperatures in the upper photic zone, followed by eutrophic and moderately warm sea surface temperatures. The long-term pattern of the warm taxa and $\mathrm{N}$ values may be related to a relative reduction in upwelling intensity and a warming trend at the sea surface over the last $560 \mathrm{Ka}$. On the other hand, the rare concentrations of $E$. huxleyi and $C$. pelagicus could be temperature-induced, whereas $C$. leptoporus and $H$. carteri do not appear to show any ecological preference.

The interval studied was divided into four parts characterised by different palaeoceanographic/palaeoatmospheric conditions as indicated by variations in the $N$ index, nannofossil accumulation rates, and coccolith-derived $\mathrm{CaCO}_{3}$. Higher $N$ values occurred during Interval 1 (MIS 14-8) during the mid-Brunhes event, suggesting a shallow nutri-thermocline and enhanced upwelling. This situation corresponds to maximum NAR and coccolith carbonate, possibly related to La Niña-like events. Interval 2 (MIS 8-6) shows high $N$ values, although with fluctuations, as a consequence of variations in the position of the nutri-thermocline. This scenario was associated with strong Trade winds and enhanced upwelling, possibly related to a La Niñalike dominant situation. Lower N values during interval 3 (MIS 5) reveal a deep nutri-thermocline, due to a reduction in the southeast Trade wind stress and a reduction in the NAR and N index. This situation is interpreted as El Niño-like conditions. The water surface regime during interval 4 (MIS 4-1) is marked by incursions of cold and nutrient-rich advected waters introduced by southeast Trade wind stress, which favoured the increase in the abundances of the upper photic species, the NAR and coccolith $\mathrm{CaCO}_{3}$ contents, and the whole scenario can be interpreted as La Niña-like dominant period.

\section{Acknowledgements}

The authors wish to thank the ODP Program Leg 202 for providing samples for calcareous nannofossil analysis. Thanks are also given to Dr. Hanno Kinkel and Mrs. Ute Schuldt (Institute of Geosciences, CAU Kiel) for their support in the SEM lab and discussions. We are also very grateful to Drs. Jeremy Young, Sebastian Meier and two anonymous reviewers for their careful revision and contributions which highly improved this paper. Financial support from the Spanish Ministerio de Educación y Ciencia, through an FPI Fellowship BES-200-0010 provided to G.E. López-Otálvaro and from Spanish projects BTE2002-04670, REN2003-08642-C02/CLI and and 
CGL2006-10593 from the Ministerio de Educación y Ciencia, and SA088/04 from the Consejería de Educación de la Junta de Castilla y León is acknowledged.

\section{References}

Ahagon, N., Tananka, Y., Ujiié, H. 1993. Florisphaera profunda, a posible nannoplankton indicador of Late Quaternary changes in sea-water turbidity at the northwestern margin of the Pacific. Marine Micropaleontology 22, 255-273.

Archer, D.E. 1991a. Equatorial Pacific calcite preservation cycles: production or dissolution. Paleoceanography 6, 561-571.

Archer, D.E. 1991b. Modelling the calcite lysocline. Journal of Gephysical Research 96, 17037-17050.

Baumann, K.-H., Freitag, T. 2004. Pleistocene fluctuations in the northern Benguela Current systems as revealed by coccolith assemblages. Marine Micropaleontology 52, 195-215.

Baumann, K.-H., Böckel, B., Frenz, M. 2004. Coccolith contribution to South Atlantic carbonate sedimentation. In: Thierstein, H.R., Young, J.R. (Eds.), Coccolithophores - from molecular processes to global impact. Springer-Verlag, Berlin-Heidelberg, pp. 367-402.

Beaufort, L., Buchet, N. 2003. Variability of specific coccolith $\mathrm{CaCO}_{3}$ weight and primary production in the western Pacific warm pool during the last glacial cycle. Geophysical Research Abstracts (European Geosciences Union) 5, 13225.

Beaufort, L., Lancelot, Y., Camberlin, P., Cayre, O., Vincent, E., Bassinot, F., Labeyrie, L. 1997. Insolation cycles as a major control of equatorial Indian Ocean primary production. Science 278, 14511454.

Beaufort, L., Bassinot, F.C., Vincent, E. 1999. Primary production response to orbitally induced variations of the Southern Oscillation in the Equatorial Indian Ocean. In: Abrantes, F., Mix, A.C. (Eds.), Reconstructing Ocean History: a Window into the Future. Kluwer Academic/Plenum, New York, pp. 245-272.

Beaufort, L., de Garidel-Thoron, T., Mix, A.C., Pisias, N.G. 2001. ENSO-like forcing on oceanic primary production during the Late Pleistocene. Science 293, 2440-2444.

Beaufort, L., de Garidel-Thoron, T., Linsley B., Oppo D., Buchet N. 2003. Biomass burning and oceanic primary production estimates in the Sulu Sea area over the last $380 \mathrm{Ky}$ and the East Asian monsoon dynamics. Marine Geology 201, 53-65.

Böeckel, B., Baumann, K.-H. 2004. Distribution of coccoliths in surface sediments of the south-eastern South Atlantic Ocean: ecology, preservation and carbonate contribution. Marine Micropaleontology 51, 301-320.

Bollmann, L., Baumann, K.-H., Thierstein, H.R. 1998. Global dominance of Gephyrocapsa coccoliths in the Late Pleistocene: Selective dissolution, evolution or global environment change. Paleoceanography 13, 517-529.

Brand, L.E. 1994. Physiological ecology of marine coccolithophores. In: Winter, A., Siesser, W.G. (Eds.) Coccolithophores. Cambridge University Press, Cambridge, U.K., pp. 39-49.

Cachão, M., Moita, M.T. 2000. Coccolithus pelagicus, a productivity proxy related to moderate fronts off Western Iberia. Marine Micropaleontology 39, 131-155.

Chavez, F.P., Barber, R.T. 1987. An estimate of new production in the equatorial Pacific. Deep-Sea Research Part A, 34, 1229-1243.

Clement , A.C., Seager, R., Cane, M.A. 1999. Orbital controls on the El Niño/Southern Oscillation and the tropical climate. Paleoceanography 14, 441-456.

Droxler, A.W., Alley, R.B., Howard, W.R., Poore, R.Z., Burckle, L.H. 2003. Unique and exceptionally long interglacial Marine Isotope Stage 11: Window into Earth Warm Future Climate. In: Droxler, A.W., Poore, R.Z., Burckle, L.H (Eds), Earth's climate and orbital eccentricity. The Marine Isotope Stage 11 question. Monograph Series 137, AGU, Washington, DC., 1-14.

Emeis, K.-C., Doose, H., Mix, A., Schulz-Bull, D. 1995. Alkenone sea surface temperatures and carbon burial at Site 846 (Eastern Equatorial Pacific Ocean): the last 1.3 M.y. In: Pisias, N.G., Mayer, L.A., Janecek, T.R., Palmer-Julson, A., van Andel, T.H. (Eds.). Proc. ODP, Sci. Results, 138: College Station, TX (Ocean Drilling Program), pp. 605-613. 
Farrell, J.W., Murray, D.W., McKenna, V.S., Ravelo, A.C. 1995. Upper ocean temperature and nutrient contrast inferred from Pleistocene planktonic foraminifer $\delta^{18} \mathrm{O}$ and $\delta^{13} \mathrm{C}$ in the Eastern Equatorial Pacific. In: Pisias, N.G., Mayer, L.A., Janecek, T.R., Palmer-Julson, A., van Andel, T.H. (Eds.). Proc. ODP, Sci. Results, 138: College Station, TX (Ocean Drilling Program), pp. 289-319.

Fedorov, A.V., Philander, G. 2000. Is El Niño Changing?. Science 288, 1197-2002.

Fiedler, P.C., Talley, L.D. 2006. Hydrography of the eastern tropical Pacific: A review. Progress in Oceanography $69,143-180$.

Flores, J.A., Sierro, F.J. 1997. Revised technique for calculation of calcareous nannofossil accumulation rates. Micropaleontology 43, 321-324.

Flores, J.A., Gersonde, R., Sierro, F.J. 1999. Pleistocene fluctuations in the Agulhas Current Retroflection based on the calcareous plankton record. Marine Micropaleontology 37, 1-22.

Flores, J.A., Bárcena, M.A. y Sierro, F.J. 2000. Ocean-surface and wind dynamics in the Atlantic Ocean off Northwest Africa during the last 140000 years. Palaeogeography, Palaeoclimatology, Palaeoecology 161, 459-478.

Flores, J.A., Marino, M., Sierro, F.J., Hodell, D.A. y Charles, C.D. 2003. Calcareous plankton dissolution pattern and coccolithophore assemblages during the last $600 \mathrm{kyr}$ at ODP Site 1089 (Cape Basin, South Atlantic): paleoceanographic implications. Palaeogeography, Palaeoclimatology, Palaeoecology 196, 409-426.

Flores, J.-A., Wei, W., López-Otálvaro, G.-E., Alvarez, C., Sierro, F.J. 2006. Data report: Tropical and equatorial calcareous nannofossil Pleistocene biostratigraphy, ODP Leg 202. In: Tiedemann, R., Mix, A.C., Richter, C., and Ruddiman, W.F. (Eds.), Proc. ODP, Sci. Results, 202: College Station, TX (Ocean Drilling Program), 1-10. doi:10.2973/odp.proc.sr.202.214.2006.

Geitzenauer. K.R., Roche, M.B., Mclntyre, A. 1977. Coccolithophore biogeography of the North Atlantic and Pacific surface sediments. In: Ramsay (Ed.). Oceanic Micropaleontology. Academic Press, New York, pp. 973-1008.

Giraudeau, J. 1992. Distribution of Recent nannofossils beneath the Benguela system: Southwest African continental margin. Marine Geology 108, 219-237.

Giradeau, J., Monteiro, P.M.S., Nikodemus, K. 1993. Distribution and malformation of living coccolithophores in the northern Benguela upwelling system of Namibia. Marine Micropaleontology 22, 93-110.

Hagelberg, T.K., Pisias, N.G., Mayer, L.A., Shackleton, N.J., Mix, A.C. 1995. Spatial variability of Late Neogene equatorial Pacific carbonate: Leg 138. In Pisias, N.G., Mayer, L.A., Janecek, T.R., Palmer-Julson, A., van Andel, T.H. (Eds.). Proc. ODP, Sci. Results, 138: College Station, TX (Ocean Drilling Program), pp. 321-336.

Hagino, K., Okada, H. 2004. Floral response of coccolithophores to progressive oligotrophication in the South Equatorial Current, Pacific Ocean. In: Shiyomi, M., Kawahata, H., Koizumi, H., Tsuda, A., Awaya, Y. (Eds.), Global Environmental Change in the Ocean and on Land. TERRAPUB, pp. 121-132.

Helly, J., Levin, L. 2004. Global distribution of naturally occurring marine hypoxia on continental margins. Deep-Sea Research I 51, 1159-1168.

Hofman, E.E., Busalacchi A.J., O'Brien J.J. 1981. Wind generation of the Costa Rica Dome. Science 214, 552-554.

Houghton, S.D., Guptha, M.V.S. 1991. Monsoonal and fertility controls on recent marginal sea and continental shelf coccolith assemblages from the western Pacific and northern Indian oceans. Marine Geology 97, 251-259.

Hovan, S.A. 1995. Late Cenozoic atmospheric circulation intensity and climatic history recorded by eolian deposition in the Eastern Equatorial Pacific Ocean, Leg 138. In Pisias, N.G., Mayer, L.A., Janecek, T.R., Palmer-Julson, A., van Andel, T.H. (Eds.). Proc. ODP, Sci. Results, 138: College Station, TX (Ocean Drilling Program), pp. 615-625.

Jansen, J.H.F., Kuijpers, A., Troelstra, S.R. 1986. A mid-Brunhes climatic event: long-term changes in global atmospheric and ocean circulation. Science, 232:619-622.

Kessler, W.S. 2006. The circulation of the eastern tropical Pacific: A review. Progress in Oceanography $69,181-217$. 
Lea, D. W., Pak, D. K., Belanger, C. L., Spero, H. J., Hall., M. A., Shackleton, N. J. 2006. Paleoclimate history of Galápagos surface waters over the last 135,000 yr. Quaternary Science Reviews 25, 1152-1157.

Liu, Z., Herbert, T. 2004. High-latitude on the Eastern Equatorial Pacific climate in the early Pleistocene epoch. Nature 427, 720-723.

Lukas, R. 1986. The termination of the Equatorial Undercurrent in the Eastern Pacific. Progress in Oceanography 16, 63-90.

Lyle, M., Murray, D.W., Finney, B.P., Dymond, J., Robbins, J.M., Brooksforce, K. 1988. The record of Late Pleistocene biogenic sedimentation in the eastern tropical Pacific Ocean. Paleoceanography 3 , 39-59.

Lyle, M., Mix. A., Pisias, N. 2002. Patterns of $\mathrm{CaCO} 3$ deposition in the eastern tropical Pacific Ocean for the last 150 kyr: Evidence for a southeast Pacific depositional spike during marine isotope stage (MIS) 2 Paleoceanography, 17(2), 1013, doi : 1 0.1029/2000PA000538.

Martínez, I., Rincón, D., Yokoyama, Y., Barrows, T. 2005. Foraminifera and coccoloithophorid assemblage changes in the Panama Basin during the last deglaciation: Response to sea-surface productivity induced by a transient climate change. Palaeogeography, Palaeoclimatology, Palaeoecology 234, 114-126.

Mitchell-Innes, B. A., Winter, A. 1987. Coccolithophores: a major phytoplankton component in mature upwelled waters off Cape Peninsula, South Africa in March, 1983. Marine Biology 95, 25-30.

Mix, A.C., Tiedemann, R., Blum, P., et al., 2003. Proc. ODP, Init. Repts., 202: College Station, TX (Ocean Drilling Program). doi:10.2973/odp.proc.ir.202.2003.

Molfino, B. R., Mclntyre, A. 1990a. Nutricline variation in the equatorial Atlantic coincident with the Younger Dryas. Paleoceanography 5, 997-1008.

Molfino, B., Mclntyre, A. 1990b. Precessional forcing of nutricline dynamics in the equatorial Atlantic. Science 249, 766-769.

Murray, D.W., Farrel, J.W., McKenna, V. 1995. Biogenic sedimentation at Site 847, Eastern Equatorial Pacific Ocean, during the past 3 My. In Pisias, N.G., Mayer, L.A., Janecek, T.R., Palmer-Julson, A., van Andel, T.H. (Eds.). Proc. ODP, Sci. Results, 138: College Station, TX (Ocean Drilling Program), pp. 429-459.

Okada, H., Honjo, S. 1973. The distribution of oceanic coccolithophorids in the Pacific. Deep-Sea Research 20, 355-374.

Okada, H., Honjo, S. 1975. Distribution of coccolithophores in marginal seas along the western Pacific ocean and in the Red Sea. Marine Biology 31, 271-286.

Okada, H., McIntyre, A. 1979. Seasonal distributions of Modern coccolithophores in the western Atlantic ocean. Marine Biology 54, 319-328.

Okada, H., Wells, P. 1997. Late Quaternary nannofossil indicators of climate change in two deep-sea cores associated with the Leeuwin Current off Western Australia. Palaeogeography, Palaeoclimatology, Palaeoecology 131, 413-432.

Paillard, D.L., Labeyrie, L., Yiou, P. 1996. Macintosh program performs time-series analysis. EOS, Transactions, American Geophysical Union, 77 (39), 379.

Pak, H., Zaneveld, J.R. 1974. Equatorial Front in the eastern Pacific ocean. Journal of Physical Oceanography 4, 570-578.

Philander, G. 1995. El Niño and La Niña. In: Pirie, R.G. (Ed.), Oceanography: contemporary readings in ocean sciences, Oxford Univ. Press, Oxford, U.K., pp. 72-87.

Philander, S.G.H., Gu, D., Halpern, D., Lambert, G., Lau, N.-C., Li, T., Pacanowski, R.C. 1996. Why the ITCZ is mostly north of the Equator. Journal of Climate 9:2958-2972.

Ravelo, A. C., Shackleton, N. J. 1995. Evidence for surface water circulation changes at ODP Site 851 in the eastern tropical Pacific. In: Pisias, N.G., Mayer, L.A., Janecek, T.R., Palmer-Julson, A., van Andel, T.H. (Eds.). Proc. ODP, Sci. Results, 138: College Station, TX (Ocean Drilling Program), pp. 503-514.

Rickaby, R.E.M., Halloran, P. 2005. Cool La Niña during the warmth of the Pliocene? Science 307, 19481952.

Scharmm, C.T. 1985. Implications of radiolarian assemblages for the Late Quaternary Paleoceanography of the eastern equatorial Pacific. Quaternary Research 25, 204-218. 
Shackleton, N.J., Berger, A., Peltier, W.R. 1990. An alternative astronomical calibration of the Pleistocene timescale based on ODP Site 677. Transactions of the Royal Society of Edinburgh: Earth Sciences 81, 251-261.

Smith, W.H.F., Sandwell, D.T. 1997. Global sea floor topography from satellite altimetry and ship depth soundings. Science 277, 1956-1962.

Snoeckx, H., Rea, D. 1994. Late Quaternary $\mathrm{CaCO}_{3}$ stratigraphy of the eastern equatorial Pacific. Paleoceanography, 9 (2): 341-352.

Thierstein, H.R., Geitzenauer; K.R., Molfino, B., Shackleton, N.J. 1977. Global synchroneity of late Quaternary coccolith datum levels: Validation by oxygen isotopes. Geology 5, 400-404.

Thomas, E., Turekian, K.K., Wei, K.-Y. 2000. Productivity control of fine particle transport to equatorial Pacific . Global Biogeochemical Cycles 14, 945-955.

Weber, M.E. 1998. Estimation of biogenic carbonate and opal by continuous non-destructive measurements in deep-sea sediments: application to the Eastern Equatorial Pacific. Deep-Sea Research I 45, 1955-1975.

Wells, P., Okada, H. 1997. Response of nannoplankton to major changes in sea-surface temperature and movements of hydrological fronts over Site DSDP 594 (south Chatham Rise, southeastern New Zealand), during the last $130 \mathrm{Kyr}$. Marine Micropaleontology 32, 341-363.

Wyrtki, K. 1981. An Estimate of Equatorial Upwelling in the Pacific. Journal of Physical Oceanography 11, 1205-1214.

Winter, A. 1982. Paleoenvironmental interpretation of Quaternary coccolith assemblages from the Gulf of Aquaba (Elat), Red Sea. Revista Española de Micropaleontología 14, 291-314.

Young, J.R. 1994. Functions of coccoliths. In: Winter, A., Siesser, W.G. (Eds.), Coccolithophores. Cambridge Univ. Press, Cambridge, U.K., pp. 63-82.

Young, J.R., Ziveri, P. 2000. Calculation of coccolith volume and its use in calibration of carbonate flux estimates. Deep-Sea Research A 47, 1679-1700. 
Paleoproductivity and coccolith-derived carbonate contribution to sediments in the Eastem Tropical Pacific over the past $925 \mathrm{Ka:}$ ODP sites 1241 and 1242

\author{
G.-E. López-Otálvaro ${ }^{a^{*}}$; J.F. Flores ${ }^{a} ;$ F.J. Sierro ${ }^{a}$ A. Mix ${ }^{b}$
}

${ }^{a}$ Department of Geology. University of Salamanca. Plaza de la Merced s/n. E-37008 Salamanca, Spain.

${ }^{b}$ College of Oceanic and Atmospheric Sciences, Oregon State University, Corvallis, Oregon, USA.

Submitted to Palaeogeography, Palaeoclimatology, Palaeoecology

Abstract

Key words

6.1. Introduction

6.2. Oceanographic setting

6.3. Materials and methods

6.3.1. Core localtion and materials

6.3.2. Calcareous nannoplankton preparation and counting techniques

6.3.3. Biostratigraphy and age model

6.3.4. Calcareous nannofossil palaeoproductivity index and coccolith-derived carbonate abundances

6.3.5. Taxonomic notes

6.4. Calcareous nannofossil preservation

6.5. Results

6.5.1. Variations in the assemblage

6.5.2. Patterns of the $\mathrm{N}$ ratio and the total $\mathrm{CAR}$

6.6. Discussion

6.6.1. The $\mathrm{N}$ ratio and coccolith-derived carbonate: preservation or palaeoproductivity profiles?

6.6.2. Glacial/interglacial nutri/thermocline variability

6.6.3. ENSO-like events

6.6.4. The mid-Brunhes event

6.6.5. Calcareous nannofossil assemblage

6.7. Conclusions

Aknowledgements

References

*Correspondence to: G.-E. López-Otálvaro (gatsbyemperatriz@usal.es) 


\section{Abstract}

Middle and late Pleistocene variations in the assemblage of calcareous nannofossils were analysed quantitatively in the Eastern Tropical Pacific (ETP) warm pool with a view to elucidating temporal changes in palaeoproductivity, the vertical migration of the nutri-thermocline and sea surface circulation at Ocean Drilling Program (ODP) sites 1241 and 1242. These sites are located under the warm water regime of the North Equatorial Countercurrent (NECC) and the Intertropical Convergence Zone (ITCZ). A good to moderate degree of calcareous nannofossil preservation was observed in most of the records and was indicative of the palaeoecological signal of the coccolith-derived carbonate proxies used. The interglacial stages at Site 1242 show less preservation of the coccolith-derived carbonate, revealing the "Pacific dissolution pattern", but Site 1241 does not exhibit this pattern. A few intervals during interglacial stages show poor preservation at Site 1242, obscuring the palaeoecological significance of the assemblage during those intervals. However, a comparative study of the temporal distribution of calcareous nannofossils and their coccolith-derived carbonate fluxes (CAR) at both sites revealed that calcareous nannofossil production outweighed dissolution even during the mid-Brunhes event, and that the effects of dissolution were enhanced when calcareous nannofossil production fell.

The $\mathrm{N}$ ratio was used to infer changes in palaeoproductivity through the relationship of selected upper photic zone dwellers (small Noelaerhabdaceae and Gephyrocapsa oceanica) versus the lower photic zone dwellers (Florisphaera profunda). High glacial $\mathrm{N}$ ratio values suggest high glacial palaeoproductivity and a shallow nutri-thermocline during times of the weakening of the warm water regime of the NECC. Lower interglacial $\mathrm{N}$ ratio values indicate low interglacial surface productivity and a nutrient-thermal stratification, suggesting a reinforcement of the warm NECC. Coccolith-derived carbonate accumulation rates (CAR) were used as a proxy for the production of biogenic carbonate. The $\mathrm{N}$ ratio and the CAR profiles follow each other closely, indicating that variations in CAR values also respond to a palaeoproductivity pattern that was high during glacial stages. Thus, the palaeoproductivity profiles reveal the typical "Pacific $\mathrm{CaCO}_{3}$ pattern". The general pattern of the palaeoproductivity proxies used here also indicates that the major variation in the nutri-thermocline occurred during MIS 5, pointing to a warmer and more oligotrophic setting in the surface waters, comparable with an El Niño-like state. The individual glacial CAR of small Noelaerhabdaceae, G. oceanica, G. caribbeanica, U. sibogae, C. leptoporus and $H$. carteri, higher during glacial stages, show the preferences of these species for more fertile water masses in the warm pool over the past $925 \mathrm{Ka}$. The CAR produced in the upper photic 
zone exceeds that produced in the lower photic zone, as revealed by lower $F$. profunda-specific CAR values, even when palaeoproductivity was lowered. Such a situation reinforces the idea that palaeoproductivity has been relatively higher in the ETP during the past $925 \mathrm{Ka}$.

Key words: Eastern Tropical Pacific, Panama Basin, coccoliths, calcareous nannofossils, palaeoproductivity, Pleistocene, Mid-Brunhes event, coccolith-derived carbonate, Site 1241, Site 1242.

\subsection{Introduction}

The close relationship between the availability of nutrients and cooler sea surface temperatures has been explained in terms of the coincidence of the nutricline and the thermocline at low latitudes (Zentara and Kamykowski, 1977; Fiedler and Talley, 2006; Pennington et al., 2006).

Calcareous nannofossils are one of the major primary producers in the ocean (Rost and Riebesell, 2004). They are abundant and generally well preserved along the sedimentary sequences recovered in the Eastern Pacific Ocean during Ocean Drilling Program (ODP) Leg 202 (Mix et al., 2003; Flores et al., 2006). Their temporal and geographical distributions and their contribution to the carbonate sedimentation in the Pacific Ocean have frequently been associated with the availability of nutrients; the displacement of nutri-thermocline depth; variations in upwelling, surface temperature and/or salinity. Such oceanographic changes are linked to wind shifting, since they control the thermocline depth, on which nutrient supply and biological production depend (Okada and Honjo, 1973; Okada and Honjo, 1975; Geitzenauer et al., 1977; Molfino and McIntyre, 1990a, b; Beaufort et al., 2001; Beaufort and Buchet, 2003; Hagino and Okada, 2004; Pennington et al., 2006; López-Otálvaro et al., 2008a, b). Thermocline shallowing in the Eastern Tropical Pacific Ocean (ETP) is driven by three processes: 1) a basin-scale eastwest thermocline tilt, which shallows in the east of the basin and deepens to the west of it (Barber and Chávez, 1983); 2) basin-scale thermocline shallowing at the gyre margins (Pennington et al., 2006), and 3) local wind-driven upwelling (Barber and Chavez, 1983; Pennington et al., 2006).

Carbonate sediments preserved in the deep sea represent an excellent climatic record in the ETP that responds to Pleistocene glacial/interglacial changes (Arrhenius, 1952; Archer, 1991a, b; Karlin et al., 1992; Farrell and Prell, 1991, Lyle et al., 2002; among others). It can be correlated along the Pacific and Indian oceans (Farrell and Prell, 1991; Bassinot et al., 1994), and it is 
strongly influenced by $\mathrm{CaCO}_{3}$ production and dissolution (Arrhenius, 1952; Archer, 1991a, b; Lyle et al., 1988; among others), leading to important changes in $\mathrm{PCO}_{2}$ (Rickaby et al., 2007). Fluctuations in the carbonate record of the Pacific Ocean have been explained in terms of two theories: 1) Variations in palaeoproductivity and changes in dissolution intensity. Palaeoproductivity suggests that the calcite/organic carbon ratio was lower due to an enhanced production of biogenic carbonate in surface waters during glacial stages with glacial/interglacial circulation remained unchanged (Arrhenius, 1952; Pedersen, 1983; Archer, 1991a, b). 2) The preservation theory holds that the dissolution intensity was stronger during interglacial rather than glacial events, and that productivity hardly changed in relation to changes in the ocean circulation in the Pacific Ocean (Berger, 1968; Farrell and Prell, 1989) or was associated with an increase in the rain ratio $\left(\mathrm{CaCO}_{3} /\right.$ organic carbon) (Berger, 1973; Thompson and Saito, 1974; Farrell and Prell, 1989).

In this paper, we quantitatively estimate the calcareous nannofossil assemblage and coccolithderived carbonate fluxes in order (1) to assess whether changes in the production of nannofossil carbonate correspond to the preservation pattern or to the palaeoecological signal, and (2) to gain insight into the evolution of the vertical movement of the nutri-thermocline, changes in palaeoproductivity, and mass fluxes in the deep-sea sediments in the ETP in order to reconstruct sea surface dynamic over the past $925 \mathrm{Ka}$ in the warm pool of the ETP.

\subsection{Oceanographic setting}

Oceanic circulation in the ETP is controlled by the Coriolis force across the equator, supporting a climatic asymmetry relative to the equator that is favored by the geometry and topography of the American continent (Philander et al., 1996). The Trades converge onto the off-equatorial Intertropical Convergence Zone (ITCZ) in the north-ETP (Philander et al., 1996) over warmer sea surface waters $\left(>28.5^{\circ} \mathrm{C}\right)$, known as the warm pool (Wang and Fiedler, 2006). This north-south ITCZ-related asymmetry is also revealed in the surface oceanic circulation (Wyrtki, 1967), lower nutrient supply, lower productivity of phytoplankton, lower chlorophyll levels, the location of the oxygen minimum zone (OMZ), lower $\mathrm{CO}_{2}$ fluxes (Pennington et al., 2006) and lower salinity (Wyrtki, 1966; Pak and Zaneveld, 1974; Archer et al., 1997; Benway and Mix, 2004; Benway et al., 2006; Kessler, 2006, Pennington et al., 2006). The Panama Basin is an area of generally high productivity (Pedersen et al., 1991), and it is also the lowest 0-100 m salinity region in the ETP as 
a consequence of high precipitation in comparison with evaporation due to water vapor transfer from the western tropical Atlantic (WTA) during the dominance of the northeast Trade winds and the influence of the northern ITCZ position during the intensification of the southeast Trade winds (Zaucker and Broecker, 1992; Benway and Mix, 2004; Leduc et al., 2007).

Oceanic circulation in the ETP is seen in two closed cells: the cell that comprises the South Equatorial Current (SEC) and the Peru Current (PC), and the cell that involves the North Equatorial Current (NEC) and the North Equatorial Countercurrent (NECC) (Baumgartner and Christensen, 1985). The warm pool is dragged in an easterly direction by the NECC between the NEC and the SEC $\left(3^{\circ} \mathrm{N}-9 \circ \mathrm{N}\right)$ and has no equivalent south of the equator (Philander, 1990), causing a shallowing of the nutri-thermocline south of the equator and a relative deepening of the nutri-thermocline north of it (Philander et al., 1996) (Fig. 6.1).

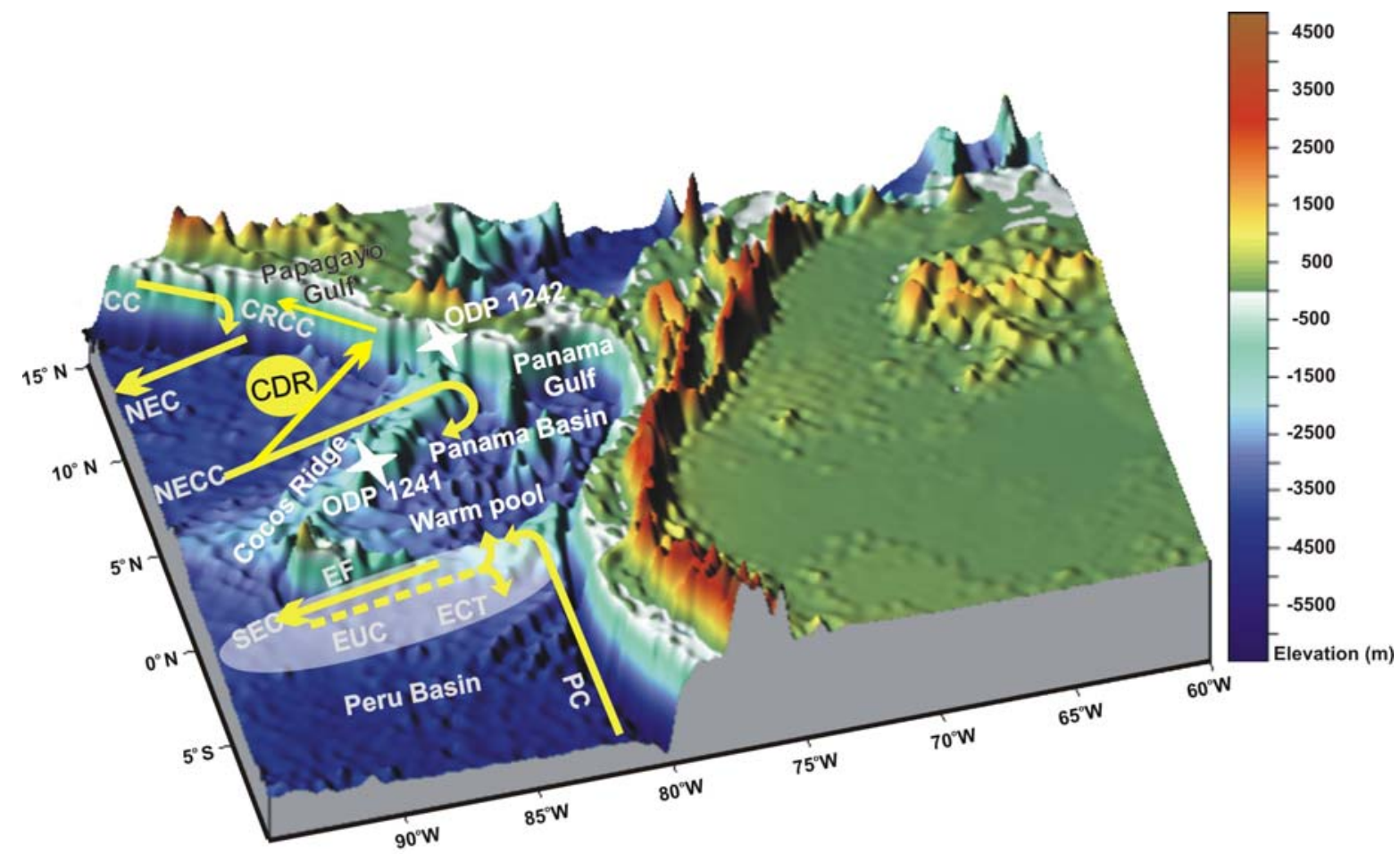

\begin{tabular}{|l|c|c|c|c}
\hline ODP Site & General location & Latitude & Longitude & Water depth \\
\hline $\mathbf{V}_{1241}$ & $\begin{array}{c}\text { Cocos Ridge } \\
\text { (Panama Basin) }\end{array}$ & $5^{\circ} 50.570^{\circ} \mathrm{N}$ & $86^{\circ} 26.676^{\prime} \mathrm{W}$ & $2027 \mathrm{~m}$ \\
$\mathbf{V}_{1242}$ & $\begin{array}{c}\text { Cocos Ridge } \\
\text { (Guatemala Basin) }\end{array}$ & $7^{\prime \prime} 51.352^{\prime} \mathrm{N}$ & $83^{\prime \prime} 36.418^{\prime} \mathrm{W}$ & $1364 \mathrm{~m}$ \\
\hline
\end{tabular}

Fig. 6.1. 3-D main surface and subsurface oceanic circulation features in the Eastern Tropical Pacific Ocean (ETP) in response to atmospheric dynamics. Colour scale highlighting major bathymetric features (taken from the topography database from Smith and Sandwell, 1997). Surface currents are indicated by solid lines, and subsurface currents are indicated by dashed lines. CC: California Current, EUC: Equatorial Undercurrent, NEC: North Equatorial Current, NECC: North Equatorial Counter Current, PC: Peru Current, SEC: South Equatorial Current. Hydrographic features: CDR: Costa Rica Dome, ECT: Equatorial Cold Tongue. 
Dominant northeast Trade winds situate the ITCZ in the southernmost position ( 2०N, during the boreal winter, Rodríguez-Rubio et al., 2003); they weaken the SEC, and they prevent the development of the NECC east of $100^{\circ} \mathrm{W}$. The California Current (CC) reaches $30 \mathrm{~N}$, supplying waters to the NEC (Wyrtki, 1966). When the ITCZ starts to return to its northernmost position during May to July, the CC is still enhanced and the NECC starts to develop. The dominance of the southeast Trade winds (8-10^N, during the boreal summer; Rodríguez-Rubio et al., 2003) situates the ITCZ in the northernmost position, favoring a stronger development of the SEC and the NECC, and a shallowing of the thermocline in the east. When the NECC reaches the Central American coast, it develops a cyclonic gyre that feeds the Costa Rica Dome and provides westward-flowing surface waters to the NEC south of $20{ }^{\circ} \mathrm{N}$. The California Current (CC) turns to drag waters in a westward direction at about $25^{\circ} \mathrm{N}$ and supplies waters to the NECC north of $20^{\circ} \mathrm{N}$ (Wyrtki, 1967).

The cyclonic circulation through the gulfs of Panama, Papagayo and Tehuantepec, the $10^{\circ} \mathrm{N}$ thermocline ridge, and the Costa Rica Dome all characterize the major oceanographic features in the northern cell of ETP. These features are characterised by a high biological production as a consequence of nutrient input (Wyrtki, 1964). Cyclonic circulation in these gulfs responds to the seasonal wind jets (the Panama, Papagayo and Tehuantepec jets, respectively), which produce upwelling, offshore transport, thermocline shallowing, and a mixing of surface waters (Wyrtki, 1964). The $10^{\circ} \mathrm{N}$ thermocline ridge is a region of thermocline shallowing (Fiedler and Talley, 2006; Pennington et al., 2006), termed the "countercurrent thermocline ridge" by Fiedler and Talley (2006), that is caused by divergence across the ITCZ (Kessler, 2006). The Costa Rica Dome represents the end of the $10^{\circ} \mathrm{N}$ thermocline ridge and is driven by the Papagayo wind jet during the boreal winter and by the NECC retroflection during the boreal summer. The NECC retroflection in the western margin of Central America is known as the Costa Rica Coastal Current (CRCC) (Pennington et al., 2006). The Costa Rica Dome is centered at 9oN, 90॰W, and has a diameter between 100-900 Km (Hofmann et al., 1981; Fiedler, 2002, Kessler, 2002, Kessler, 2006, Pennington et al., 2006).

The warm pool is dragged by the warm NECC (Wyrtki, 1966; Pak and Zaneveld, 1974; Archer et al., 1997; Kessler, 2006, Pennington et al., 2006). The southern boundary of the warm pool is the Equatorial Front, which is a thermal and convergent front influenced by the SEC that splits the region characterised by lower temperatures, higher salinity, higher nutrients, higher $\mathrm{CO}_{2}$, and higher chlorophyll concentrations known as the Equatorial Cold Tongue (ECT) (Fig. 6.1). 
Another mode of climate variability in the Pacific Ocean is the El Niño Southern Oscillation (ENSO) system. The ENSO is a basin-scale phenomenon that occurs irregularly on interannual time scales (in the order of 1-10 years) and outweighs seasonal variability. The ENSO is produced by variations in surface winds, which in turn result in interannual fluctuations in sea surface temperatures (Philander, 1995; Wang and Fiedler, 2006). The interannual variability of the ENSO system is high along the equatorial divergence system and in the Peru, Ecuador and California coastal upwellings, and low in the subtropical gyres and in the warm pool in the ETP. The ENSO varies between the warm and the cool phases known as El Niño and La Niña.

El Niño causes a reduction in surface pressure, a relaxation of the winds, a weakening of the westward currents, the eastward extension of the warm sea surface water, and hence, the suppression of upwelling and convection over the equator (Philander, 1995; Koutavas and LynchStieglitz 2003). La Niña follows El Niño, and conversely, produces large differences in surface pressure, a strengthening of the winds and the currents, and an abnormal intensification of equatorial upwelling (Philander, 1995). Disturbances in the biological and geochemical cycles are also associated with the ENSO system, related to alterations in production in the euphotic zone and consequently to carbon cycle sequestration at ENSO scales (Turk et al., 2001b). The reduction in the supply of nutrients in sea surface waters correlates well with the deepening of the nutri-thermocline, the warming of the ocean surface, and a reduction in biological productivity, and consequently with a large decrease in the export of carbon. Conversely, the increase in the availability of nutrients in the euphotic zone is coherent with the shallowing of the nutrithermocline, a strong reduction in sea surface temperatures, an increase in productivity, and hence, a large increase in the export of carbon during La Niña events (Bjerknes, 1969; Philander, 1990; Turk et al., 2001a, b). As an example, the biological effects of El Niño during 1982-1983 and 1997-1998 can be seen in data related to increases in sea surface temperatures (up to $9^{\circ} \mathrm{C}$ ), a nutri-thermocline deepening, and hence a reduction in primary production and chlorophyll, changes in higher trophic level organisms and a reduction of 30\% in carbon export (Barber and Chávez, 1986; Dessier and Donguy, 1987; Sánchez et al., 2000; Turk et al., 2001a, b; Wang and Fiedler, 2006). Orbital scale data show similar but more intense perturbations of the background state, which is especially significant as regards the depth of the nutri-thermocline and biological production. ENSO variability has been proposed to be linked to orbital and millennial scale fluctuations in the Tropical Pacific Ocean (Clement et al. 1999, 2000; Beaufort et al., 2001; Turney et al., 2004; Álvarez, 2005; López-Otálvaro et al., 2008a; Pena et al., 2008). 


\subsection{Materials and Methods}

\subsubsection{Core location and materials}

The sedimentary sequences from sites 1241 and 1242 were drilled during the Ocean Drilling Program (ODP) Leg 202. The complete sedimentary sequences were ascertained by means of composite sections on the basis of physical properties such as colour, magnetic susceptibility, and gamma-ray attenuation porosity evaluator (GRAPE; Mix et al., 2003).

Material from Site $1241\left(5^{\circ} 50.570^{\prime} \mathrm{N}, 86^{\circ} 26.676^{\prime} \mathrm{W}\right.$ at $2027 \mathrm{~m}$ water depth) was recovered from the north flank of the Cocos Ridge in the Panama Basin (Fig. 6.1). The sequence is $447.06 \mathrm{mcd}$ (meters of composite depth) long and spans the Late Miocene-Holocene interval (Mix et al., 2003). The sediments consist of clayey foraminiferal-nannofossil ooze, mainly dominated by nannofossils $(30-60 \%)$. The contributions of foraminifers $(25-45 \%)$, diatoms $(<12 \%)$ and siliciclastics (10-20\%) are also recorded in the sequence (Mix et al., 2003). The upper $17 \mathrm{mcd}$, spanning the past $830 \mathrm{Ka}$, were analysed for calcareous nannofossil studies (Fig. 6.1).

Site $1242\left(7^{\circ} 51.352^{\prime} \mathrm{N}, 8^{\circ} 36.418^{\prime} \mathrm{W}\right)$ was drilled into a graben on the crest of the Cocos Ridge, intersected by the Mesoamerican Trench in the Panama Basin at a water depth of $1364 \mathrm{~m}$ (Fig. 6.1). The sedimentary sequence is $287.74 \mathrm{mcd}$ long and spans the interval from the middle Miocene to the Holocene. Calcareous nannofossils form the dominant biogenic component of the sediment $(30 \%$ to $70 \%)$ and the siliciclastic content derived from the Central America continent gradually increases from the Pleistocene to the Holocene (Mix et al., 2003). The present work addressed the upper $108.01 \mathrm{mcd}$ at Site 1242 which comprises an interval spanning the past 925 Ka (Fig. 6.1).

\subsubsection{Calcareous nannoplankton preparation and counting tec hniques}

Bulk sediment samples were collected every 0.2 to 0.7 meters $(\sim 1.2$ to $7 \mathrm{Ka})$ and treated following the decantation technique outlined in Flores and Sierro (1997). This technique allows estimations of the absolute abundances of coccoliths (liths $\left.{ }^{*} \mathrm{~g}^{-1}\right)$. Initially, $0.2 \mathrm{gr}$ of dry bulk sediment were diluted and sonicated for a few seconds in $10 \mathrm{ml}$ of buffered distilled water. Then, $0.1 \mathrm{ml}$ of this diluted sediment solution was pipetted onto a Petri-dish containing a cover slip at the bottom, together with a solution of buffered distilled water and unflavoured gelatin. This 
solution guarantees homogeneous distribution of the sediment on the slide. After 12 to 24 hours, the cover slip was dried at $40^{\circ} \mathrm{C}$ for a few seconds and mounted on a slide with Canada balsam.

All specimens were identified and counted at species level using a polarized microscope at 1000X magnification. 120 samples corresponding to Site 1241 and 235 samples to Site 1242 were analysed and more than 500 coccoliths (autochthonous and reworked) were counted on each slide to assess quantitatively the absolute and relative coccolith abundances. Reworked Miocene, Pliocene and Pleistocene nannofossils were identified in the same visual fields, but they were always rare. Critical identifications were confirmed by Scanning Electron Microscopy (SEM) analysis on selected samples carried out at the Geological Institute at the University of Kiel.

\subsubsection{Biostratigraphy and age model}

The time-scale of Site 1242 is primarily based on the $\delta^{180}$ stratigraphy performed on fossil shells of a planktonic foraminifer - Globigerinoides ruber white - and visual comparison was made with the $\delta^{180}$ stratigraphy established by Shackleton et al. (1990) at Site 677. Stable isotope analyses were carried out by Benway and Mix (2004) for the upper $30 \mathrm{Ka}$ and Mix et al. (personal communication, 2005) for the interval between 30-925 Ka (Fig. 6.2). The $\delta^{18} \mathrm{O}$ age-depth assigned to the calcareous nannofossil markers at Site 1242 indicate significant reliability and reproducibility with respect to previous data, presenting an improved biostratigraphic framework that was related to palaeomagnetic information of the Brunhes/Matuyama Boundary (Mix et al., 2003). Owing to the absence of $\delta^{18} 0$ data at Site 1241, the biochronological outline from Site 1242 was provided as a possibility of a chronological framework at Site 1241 based on nannofossil biostratigraphy and the Brunhes/Matuyama Event (Table 6.1 and Fig. 6.2).

The Pleistocene sediments at sites 1241 and 1242 afforded further information about a series of calcareous nannofossil events previously reported by Flores et al. (2006). Here, additional samples located close to the most significant bioevents were examined at both sites in order to improve the biostratigraphic resolution. The last-occurrence events of $R$. asanoi and $P$. lacunosa were determined on the basis of their last consistent occurrence higher than $1 \%$. The individual coccoliths of $R$. asanoi are not consistent in the sequence studied at Site 1241 and comprise less than $1 \%$ of the assemblage. We suggest that the specimens found at Site 1241 correspond to reworked individuals, indicating that the sequence is younger than $880 \mathrm{Ka}$ (Fig. 6.2 and Table $6.1)$. 


\begin{tabular}{lcc}
\hline Site 1241 & mcd & Ka \\
\hline & 0 & 0 \\
LCOD G. caribbeanica & 5.3 present work & 254.63 \\
FAD E. huxleyi & 6.25 present work & 286.91 \\
LAD P. lacunosa & 9.7 present work & 459.62 \\
FCOD G. caribbeanica & 12.55 present work & 611.92 \\
Brunhes/Matuyama & 14.85 Mix et al. (2003) & 780 \\
LAD R. asanoi & 20.41 Flores et al. (2006) & 903.49 \\
\hline & & \\
& \multicolumn{2}{c}{ mcd } \\
\hline Site 1242 & Ka \\
\hline & & \\
LCOD G. caribbeanica & 30.34 present work & 254.63 \\
FAD E. huxleyi & 33.73 Flores et al. (2006) & 286.91 \\
LAD P. lacunosa & 52.37 present work & 459.62 \\
FCOD G. caribbeanica & 70.76 present work & 611.92 \\
Brunhes/Matuyama & 91 Mix et al. (2003) & 780 \\
LAD R. asanoi & 104.98 present work & 903.49 \\
\hline
\end{tabular}

Table 6.1. Calcareous nannofossil biostratigraphic events from sites 1241 and 1242 over the past $925 \mathrm{Ka}$. (LCOD: Last Common Occurrence Datum; FAD: First Appearance Datum; LAD: Last Appearance Datum; FCOD: First Common Occurrence Datum).

The Last Appearance Datum (LAD) of $R$. asanoi was dated at the boundary of Marine Isotope Stage (MIS) 22/21 (880 Ka) by Raffi (2002), i.e., slightly above ours. The Last Common Occurrence Datum (LCOD) of G. caribbeanica late in MIS 8, the LAD of $P$. lacunosa at $458 \mathrm{Ka}$ (MIS 12) and the First Appearance Datum (FAD) of E. huxleyi at $268 \mathrm{Ka}$ (MIS 8) were dated by Thierstein et al. (1977); i.e., slightly above ours. The timing of the First Common Occurrence Datum (FCOD) of G. caribbeanica is documented here at MIS 15, although it has been variously reported between MIS 15 to 12 (Flores et al., 1999; Bollman et al., 1998; Baumann and Freitag, 2004; Álvarez, 2005; López-Otálvaro et al., 2008a; among others). The acme of $E$. huxelyi was not identified at sites 1241 and 1242 and has been related to a high abundance of $G$. oceanica in eutrophic waters from the tropical oceans (Okada and Honjo, 1975; Houghton and Guptha, 1991; López-Otálvaro et al., 2008a) or was controlled mainly by temperature (López-Otálvaro et al., 2008a).

Earlier studies have demonstrated that fluctuations in the biogenic carbonate content are isochronous in the ETP; they follow glacial/interglacial variability and may be used as a stratigraphic tool (Arrhenius, 1952; Farrell and Prell, 1991; Thompson and Saito, 1974). The 


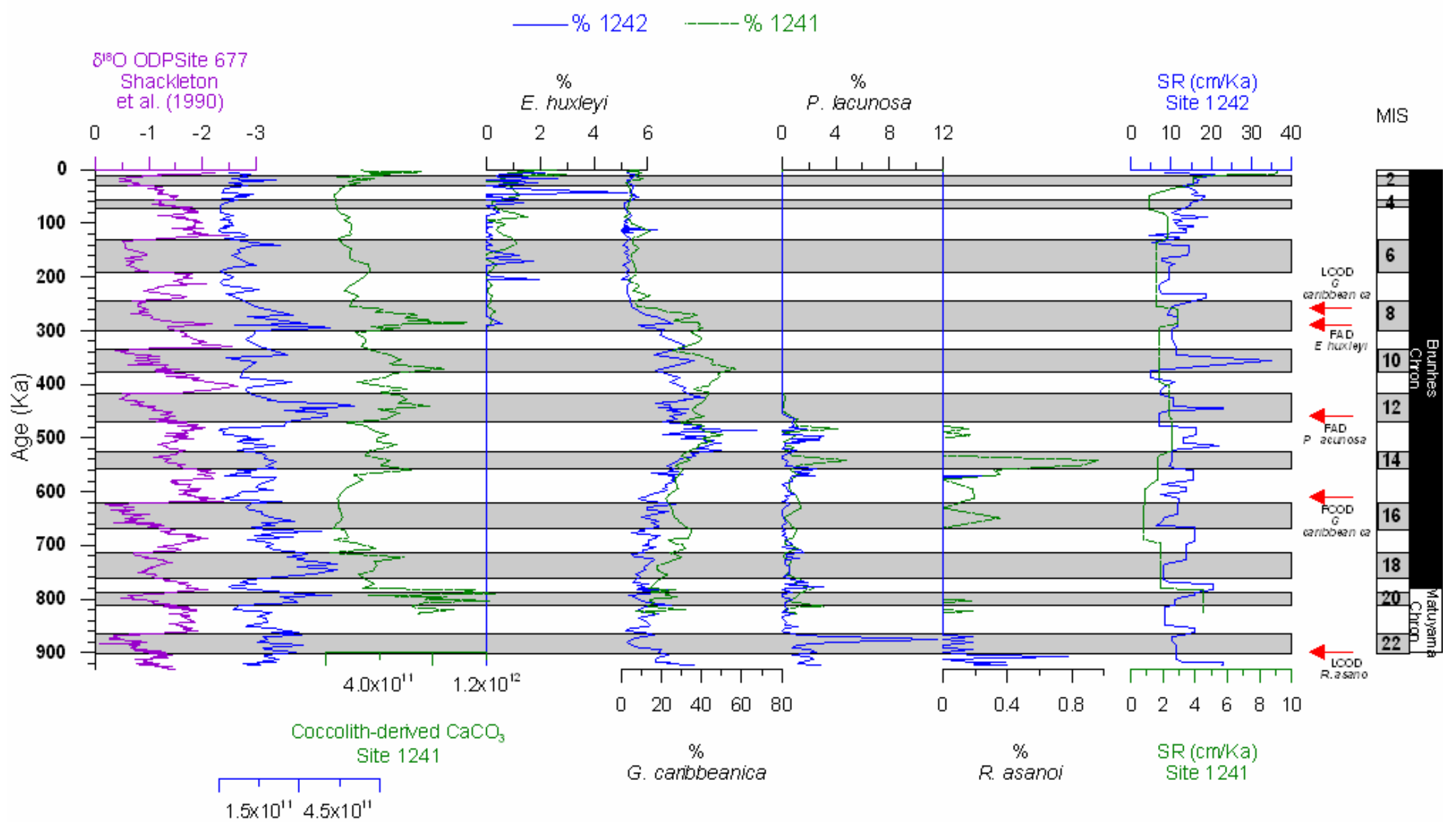

Coccolith-derived $\mathrm{CaCO}_{3}$

$$
\text { Site } 1242
$$

Fig. 6.2. Oxygen Isotope Stack from Site 677 compared to sedimentation rates and biostratigraphic events identified at sites 1241 and 1242: FAD (First Appearance Datum) of Emiliania huxleyi, LCOD (Last Common Occurrence Datum) of G. caribbeanica, LAD (Last Appearance Datum) of Pseudoemiliania lacunosa, FCOD (First Common Occurrence Datum) of G. caribbeanica and LAD of Reticulofenestra asanoi. Grey bars indicate glacial isotope stages 2 through 22 . MIS: Marine Isotope Stage. CAR: coccolith-derived carbonate accumulation rates $\left(\mathrm{pg}^{*} \mathrm{Cm}^{2 *} \mathrm{Ka}^{-1}\right)$. 
isotopic chronology recorded for the nearby Site 1242 was also used for Site 1241 by correlation of the mass flux records of both sites. The calcareous nannofossil biostratigraphy and the palaeomagnetic Brunhes/Matuyama event at Site 1241 confirm the validity of this correlation analysis (Table 6.1 and Fig. 6.2).

\subsubsection{Calcareous nannofossil palaeoproductivity index and coccolith-derived carbonate abundances}

We used a quantitative methodology to estimate the composition of the calcareous nannofossil assemblage, variations in productivity, and coccolith-derived carbonate accumulation rates (CAR) during the Pleistocene. Estimations of palaeoproductivity were obtained by means of a modification of the $\mathrm{N}$ index (modified from Flores et al., 2000; López-Otálvaro et al., 2008a, b). This index provides the relationship between selected upper (Gephyrocapsa aperta, G. ericsonii, G. oceanica and Emiliania huxleyi) and lower photic zone dwellers (Florisphaera profunda); which increases roughly in proportion to the relative abundance of the upper photic zone dwellers, opposite to the case of the $F$. profunda-dominated nannofossil assemblage. A deepening of the nutri-thermocline produces a strong stratification of the euphotic zone and hence, decreases in productivity in sea surface waters and an increase in the relative abundance of $F$. profunda. A shallowing of the nutri-thermocline results in an increase in the productivity in the sea surface water and a drop in the percentages of $F$. profunda. The relative abundance of $F$. profunda serves to monitor the variation in sea surface water productivity and therefore the depth of the nutri-thermocline (Molfino and McIntyre, 1990a, b). High values of the $\mathrm{N}$ ratio indicate increased upwelling intensity, characterising a shallowing nutri-thermocline, while low values of the $\mathrm{N}$ ratio point to evidence of a deeper nutri-thermocline and a strong stratification of the surface waters.

A close relationship between palaeoproductivity at the ocean surface with vertical particle fluxes has been reported (Steinmetz, 1994). Since calcareous nannoplankton is one of the main primary producers and carbonate contributors in the Pleistocene sediments of the ETP, mass coccolith fluxes would be a viable candidate for explaining the variability of their biogenic component and their relationship to biological production. The CAR is the burial flux in terms of mass per unit area per unit time $\left(\mathrm{pg}^{*} \mathrm{~cm}-{ }^{2 *} \mathrm{Ka}-{ }^{-1}\right)$. The relative contribution to carbonate deposition by calcareous nannofossils may provide signals for interpreting CAR values. Taxa count data provide an important basis for the estimation of coccolith-derived carbonate $\left(\mathrm{pg}^{*} \mathrm{~g}^{-1}\right)$ and hence for CAR values. To estimate the coccolith-derived carbonate, we used the technique outlined by Young 
and Ziveri (2000), which includes species-specific mean coccolith length $\left(\mu \mathrm{m}^{3}\right.$; see the Appendix $B)$, the constant dependent on the shape $\left(k_{\mathrm{s}}\right)$, the calcite density $\left(2.7 \mathrm{pg}^{*} \mu \mathrm{m}^{-3}\right.$; see section 3.5 , Table 6.2 and the Appendix B) and the absolute abundances of coccoliths. The total coccolithderived carbonate was broken down into percentages of different coccolithophoral species, and the CAR values were measured on the basis of the coccolith-derived carbonate values, sedimentation rates (cm/Ka), and the GRAPE densities obtained by Mix et al. (2003).

\subsubsection{Taxonomic notes}

The species identified mainly belong to the family of Noelaerhabdaceae and include the genera Gephyrocapsa, Reticulofenestra, Pseudoemiliania and Emiliania (Thierstein et al., 1977; PujosLamy, 1977; Wei, 1993; Raffi et al., 1993; Weaver and Thomson, 1993; Flores et al., 2003; López-Otálvaro et al., 2008a, b). Noelaerhabdaceae placoliths larger than $3 \mu \mathrm{m}$ are represented by Gephyrocapsa oceanica, G. muellerae, Reticulofenestra asanoi, Pseudoemiliania lacunosa. Placoliths smaller than $3 \mu \mathrm{m}$ (known as small Noelaerhabdaceae) are represented by Emiliania huxleyi and G. ericsonii. Small Noelaerhabdaceae also include G. aperta species, whose size is smaller than $2.5 \mu \mathrm{m}$ and which has a closed central area. Individuals of placoliths $>2.5 \mu \mathrm{m}$ with a closed central area were recognised as $G$. caribbeanica. Different sizes of $G$. oceanica were identified in the sedimentary record (medium Gephyrocapsa 3-4 $\mu \mathrm{m}$, and large Gephyrocapsa 4$5 \mu \mathrm{m})$ as regards coccolith-derived carbonate estimations, but these sizes were later lumped as Gephyrocapsa oceanica for palaeoecological purposes (Appendix B). Taxonomic descriptions and equivalences related to the Gephyrocapsa complex were adopted from Flores et al. (2000) are summarised in the Appendix B. R. asanoi species correspond to coccoliths of $>6.5 \mu \mathrm{m}$, subcircular or broadly elliptical. More strongly calcified species such as Calcidiscus leptoporus and Helicosphaera carteri show a continuous record along ODP Site 1242, but in low proportions. Different sizes of these calcified species were also recognised in order to optimize estimations of coccolith-derived carbonate (small $<5 \mu \mathrm{m}$, medium 5-8 $\mu \mathrm{m}$, large $>8 \mu \mathrm{m}$; Knappertsbusch et al., 1997), although their significance at our site is not clear. However, C. leptoporus is mainly represented by the medium morphotype (5-8 $\mu \mathrm{m})$ of Knappertsbusch et al. (1997), and $H$. carteri is mainly represented by the medium $(5-8 \mu \mathrm{m})$ and larger morphotypes $(>8 \mu \mathrm{m})$ of Knappertsbusch et al. (1997). Although specimens of Coccolithus pelagicus were always rare, diverse sizes were identified (6-9 $\mu \mathrm{m}, 10-13 \mu \mathrm{m}$ and 14-16 $\mu \mathrm{m}$; Parente et al., 2004; Narciso et al., 2006) for coccolith-derived carbonate estimation purposes, the smallest size (6-9 $\mu \mathrm{m})$ being the most common morphotype. The warm taxa are mainly represented by Umbilicosphaera 


\begin{tabular}{|c|c|c|c|c|c|}
\hline Species & $K s$ & Ks source & mean length & $\begin{array}{l}\text { Vol at mean } \\
\text { length }(\mu \mathrm{m}) 3\end{array}$ & $\begin{array}{l}\text { Coccolith } \mathrm{CaCO}_{3} \\
\text { at mean length } \\
(\mathrm{pg})\end{array}$ \\
\hline Calcidiscus leptoporus & 0.080 & Young and Ziveri, 2000 & 7.0 & 27.440 & 74.088 \\
\hline Emiliania huxleyi & 0.020 & Young and Ziveri, 2000 & 2.5 & 0.313 & 0.844 \\
\hline Florisphaera profunda & 0.040 & Young and Ziveri, 2000 & 3.3 & 1.437 & 3.881 \\
\hline Gephyrocapsa caribbeanica & 0.060 & $\begin{array}{l}\text { Adopted from G. oceanica's Ks } \\
\text { maximum value }\end{array}$ & 3.0 & 1.620 & 4.374 \\
\hline small Noerlabdaceae (G. ericsonii and G. aperta) & 0.050 & Adopted from G. ericsonii & 2.0 & 0.400 & 1.080 \\
\hline Gephyrocapsa muellerae & 0.050 & Young and Ziveri, 2000 & 3.0 & 1.350 & 3.645 \\
\hline Gephyrocapsa oceanica & 0.050 & Young and Ziveri, 2000 & 3.5 & 2.144 & 5.788 \\
\hline Large Gephyrocapsa & 0.050 & Adopted from G. oceanica & 4.5 & 4.556 & 12.302 \\
\hline Helicosphaera carteri & 0.050 & Young and Ziveri, 2000 & 10.0 & 50.000 & 135.000 \\
\hline Pseudoemiliania lacunosa & 0.040 & $\begin{array}{l}\text { Adopted from E. huxleyi type A } \\
\text { normally calcified }\end{array}$ & 4.5 & 3.645 & 9.842 \\
\hline \multicolumn{6}{|l|}{ Warm taxa: } \\
\hline Calciosolenia murrayi & 0.030 & Young and Ziveri, 2000 & 3.5 & 1.286 & 3.473 \\
\hline Neosphaera coccolithomorpha & 0.015 & Young and Ziveri, 2000 & 6.5 & 4.119 & 11.122 \\
\hline Oolithotus cavum & 0.070 & Adopted from O. cavum & 4.0 & 4.480 & 12.096 \\
\hline Oolithotus fragilis & 0.070 & Young and Ziveri, 2000 & 5.0 & 8.750 & 23.625 \\
\hline Pontosphaera spp. & 0.050 & $\begin{array}{l}\text { Adopted from H. carteri } \\
\text { (Zygodiscales) }\end{array}$ & 8.0 & 25.600 & 69.120 \\
\hline Rhabdosphaera clavigera & 0.025 & Young and Ziveri, 2000 & 8.0 & 12.800 & 34.560 \\
\hline Syracosphaera spp. & 0.030 & Adopted from S. pulchra & 4.0 & 1.920 & 5.184 \\
\hline Umbellosphaera spp. & 0.010 & Young and Ziveri, 2000 & 4.0 & 0.640 & 1.728 \\
\hline Umbillicosphaera sibogae foliosa & 0.060 & Young and Ziveri, 2000 & 4.0 & 3.840 & 10.368 \\
\hline Umbillicosphaera sibogae hulburtiana & 0.050 & $\begin{array}{l}\text { Adopted from U. sibogae } \\
\text { sibogae }\end{array}$ & 3.5 & 2.144 & 5.788 \\
\hline Umbillicosphaera sibogae sibogae & 0.050 & Young and Ziveri, 2000 & 4.0 & 3.200 & 8.640 \\
\hline
\end{tabular}

Table 6.2. Summary of coccolith shape constants used $\left(k_{s}\right)$ and carbonate coccolith calculations for each species (mean length) at sites 1241 and 1242. 
sibogae suggesting that its statistical behavior should be analysed. Other species, such as Calciosolenia murrayi, Neosphaera coccolithomorpha, Oolithotus spp., Pontosphaera spp., Rhabdosphaera clavigera, Syracosphaera spp. and Umbellosphaera spp., Umbilicosphaera foliosa, Umbilicosphaera hulburtiana (Hiramatsu and De Deckker, 1997; Böeckel and Baumann, 2004), belong to the warm taxa but were only considered for coccolith-derived carbonate estimations owing to their lower abundances (below $2 \%$ ). F. profunda has a mean size of around $3.3 \mu \mathrm{m}$. A complete list of the taxa identified at this site is provided in the Appendix A.

\subsection{Calcareous nannofossil presenvation}

The preservation of calcareous nannofossils was estimated qualitatively under Light Microscopy, defining a ranking that indicates the overall degree of sample preservation based on differential resistance of coccolith species to dissolution. The ranking was scaled from good (6), to moderate (4) and poor (2) preservation. Barren samples are marked with (0). This method is reproducible and reliable and has been formulated in previous ODP scientific publications (Raffi and Flores, 1991; Gersonde et al., 1999; Flores et al., 2006). Three extra-dissolution levels with intermediate features (between good to moderate, moderate to poor, and poor to barren samples) were introduced to refine the pattern (Table 6.3).

\begin{tabular}{|c|c|c|}
\hline Preservation ranking & Scale & Characteristics \\
\hline Good & 6 & Specimens exhibit little or no dissolution \\
\hline Good to moderate & 5 & $\begin{array}{l}\text { Specimens exhibit slight to moderate evidence of dissolution or } \\
\text { fragmentation. Diagnostic morphology is fully preserved }\end{array}$ \\
\hline Moderate & 4 & $\begin{array}{l}\text { Specimens exhibit moderate evidence of dissolution but all specimens can } \\
\text { be identified at specific level }\end{array}$ \\
\hline Moderate to poor & 3 & $\begin{array}{l}\text { Primary morphological characteristics partly altered. Nearly all specimens } \\
\text { are identified at specific level }\end{array}$ \\
\hline Poor & 2 & $\begin{array}{l}\text { Stronger dissolution and alteration of morphological characteristics. The } \\
\text { specimens are difficulty identified at specific level }\end{array}$ \\
\hline Very poor & 1 & $\begin{array}{l}\text { Species exhibit extrem dissolution and alteration of morphological } \\
\text { characteristics. The specimens identified correspond to G. oceanica } \\
\text { species. }\end{array}$ \\
\hline Barren & 0 & Coccollith-derived $\mathrm{CaCO}_{3}$ free sediments \\
\hline
\end{tabular}

Table 6.3. Preservation ranking of the calcareous nannofossils in this study.

The overall preservation pattern is moderate to good at both sites, and it is more marked at Site 1241 than at Site 1242. The moderate degree of preservation is supported by the identification of 
the most delicate specimens at species level throughout the record. Preservation declines slightly towards the top of the sites. Site 1242 records a glacial/interglacial pattern in the variability of preservation, indicating good preservation during glacial stages and moderate preservation during interglacial periods. This preservation pattern in turn results in the "Pacific $\mathrm{CaCO}_{3}$ dissolution profile" at Site 1242, but this pattern was not observed at Site 1241. Site 1242 also shows a clearly deteriorated preservation in a few intervals, such as the end of MIS 15; the middle of MIS 13; the end of MIS 7, and along MIS 5 (except MIS 5.3) (Fig. 6.3).

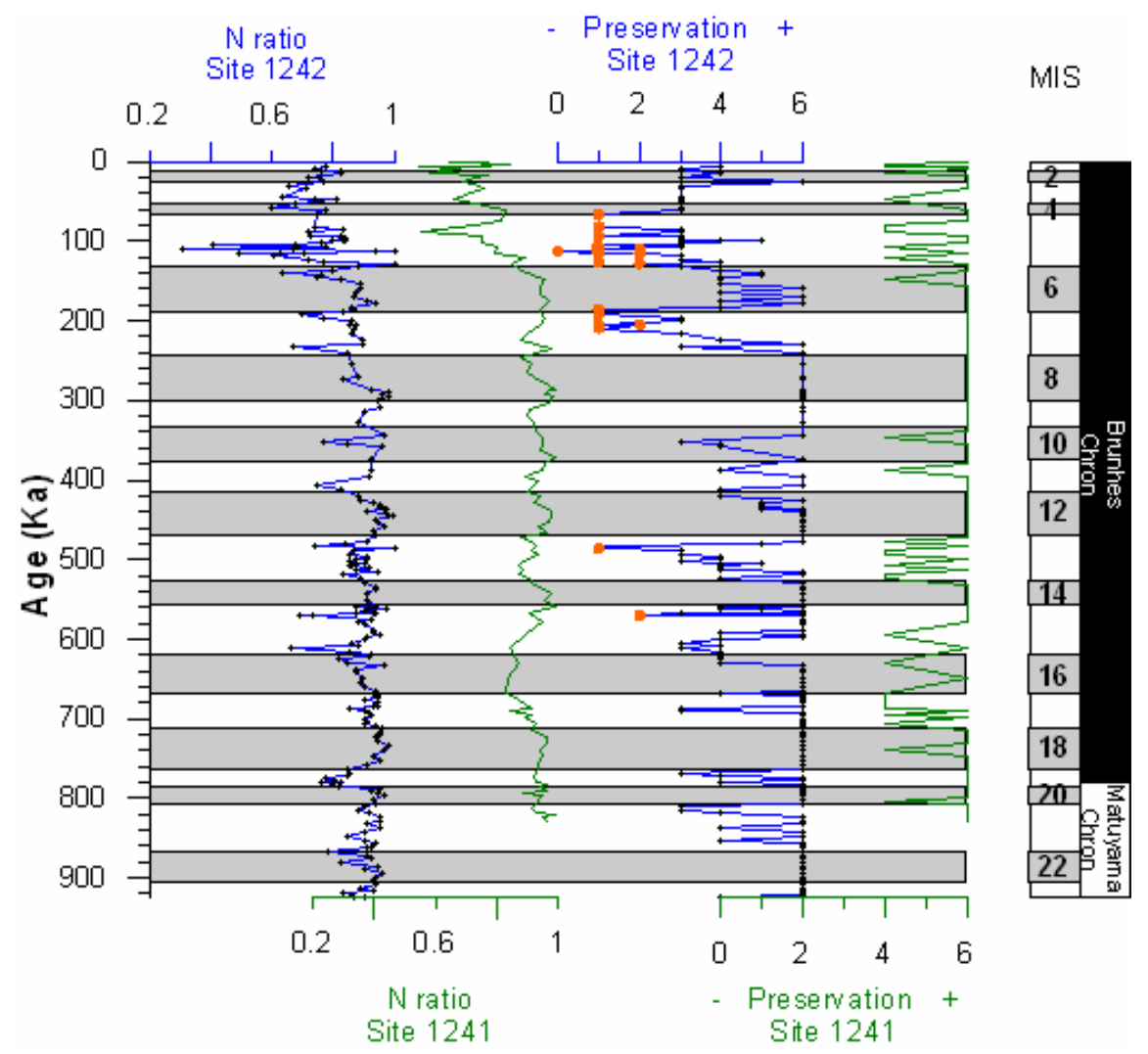

Fig. 6.3. Review of major bioproductivity proxies at sites 1241 and 1242: the $\mathrm{N}$ ratio (proportion of small Noelaerhabdaceae and G. oceanica to $F$. profunda) and the preservation pattern. Grey bars indicate glacial isotope stages 1 through 22. Orange circles in the preservation profile indicate the major dissolution points (poor preservation and barren samples). MIS: Marine Isotope Stage.

\subsection{Results}

\subsubsection{Variations in the assemblage}

Taxa such as the small Noelaerhabdaceae, G. oceanica, G. caribbeanica and Florisphaera profunda are the dominant floral constituents in the sections studied, showing higher relative abundances between $30 \%$ and $70 \%$. F. profunda is recorded at higher relative abundances at Site 1242 than at Site 1241. U. sibogae, C. leptoporus and $H$. carteri are recorded as subordinated species in terms of relative abundances (below $8 \%$ ). C. pelagicus is a taxon 
considered as a minor species in the assemblage (proportions of less than 1\%), but its appearance is more regular between 925 and $420 \mathrm{Ka}$ (MIS 22-12), and more marked at Site 1242 than at Site 1241 (Fig. 6.4).

The major contribution to the carbonate content at both sites (between $1.2 \times 10^{11}$ to $8 \times 10^{11} \mathrm{pg}^{\star} \mathrm{Ka}$ ${ }^{1 *} \mathrm{Cm}^{-2}$ ) is controlled by the most calcified taxa, such as, G. oceanica (up to $75 \%$ ), H. carteri (up to $60 \%$ ) and G. caribbeanica (up to 50\%). The mean contribution of C. leptoporus, the small Noelaerhabadaceae, F. profunda, U. sibogae and C. pelagicus has been lower during the last $925 \mathrm{Ka}$ (below 25\%). F. profunda-specific CAR values are relatively constant, with some fluctuations, and do not follow an increasing pattern over the past $283 \mathrm{Ka}$. The carbonate contribution of the small Noelaerhabdaceae is lower in spite of their dominance in the assemblage and is comparable to that of $F$. profunda. The carbonate contribution of $C$. pelagicus is particularly noteworthy across MIS 14-22 (below 4\%) despite its lowest relative abundance (Figs. 6.5, 6.6).

The species-specific CAR values of the small Noelaerhabdaceae, G. oceanica, G. caribbeanica, $U$. sibogae and $C$. leptoporus reveal higher peaks during glacial stages as compared with their proportions, which do not follow the glacial/interglacial pattern. The increases in the speciesspecific CAR values of the most calcified species - G. oceanica, G. caribbeanica, C. leptoporus and $H$. carteri - are not influenced by selective dissolution, as was confirmed by the good to moderate preservation of the calcareous nannofossils at both sites. The individual CAR values of F. profunda do not reflect preferences during glacial or interglacial events. The species-specific CAR profiles generally show higher values at Site 1241 than at Site 1242 in spite of the lower sedimentation rates at Site 1241 than at Site 1242 (Figs. 6.3, 6.5).

\subsubsection{Pattems of the $\mathbf{N}$ ratio and the total CAR}

The results obtained show that the $\mathrm{N}$ ratio and the total CAR are generally high. The $\mathrm{N}$ ratio and CAR profiles correlate well, indicating a glacial/interglacial trend, with maxima during glacial periods, showing the "typical Pacific $\mathrm{CaCO}_{3}$ profile". The CAR profile records much larger amplitude oscillations than those of the $\mathrm{N}$ ratio. The highest CAR values were estimated at Site 1241 than at Site 1242. Both, $N$ ratio and CAR records also show a distinctive pattern between 925-283 Ka (i.e. early MIS 22 to MIS 8), 283 to $128 \mathrm{Ka}$ (i.e. middle MIS 8 to MIS 6) and the 128 Ka to the Holocene (i.e. MIS 5 to MIS 1) (Figs. 6.2, 6.3). 


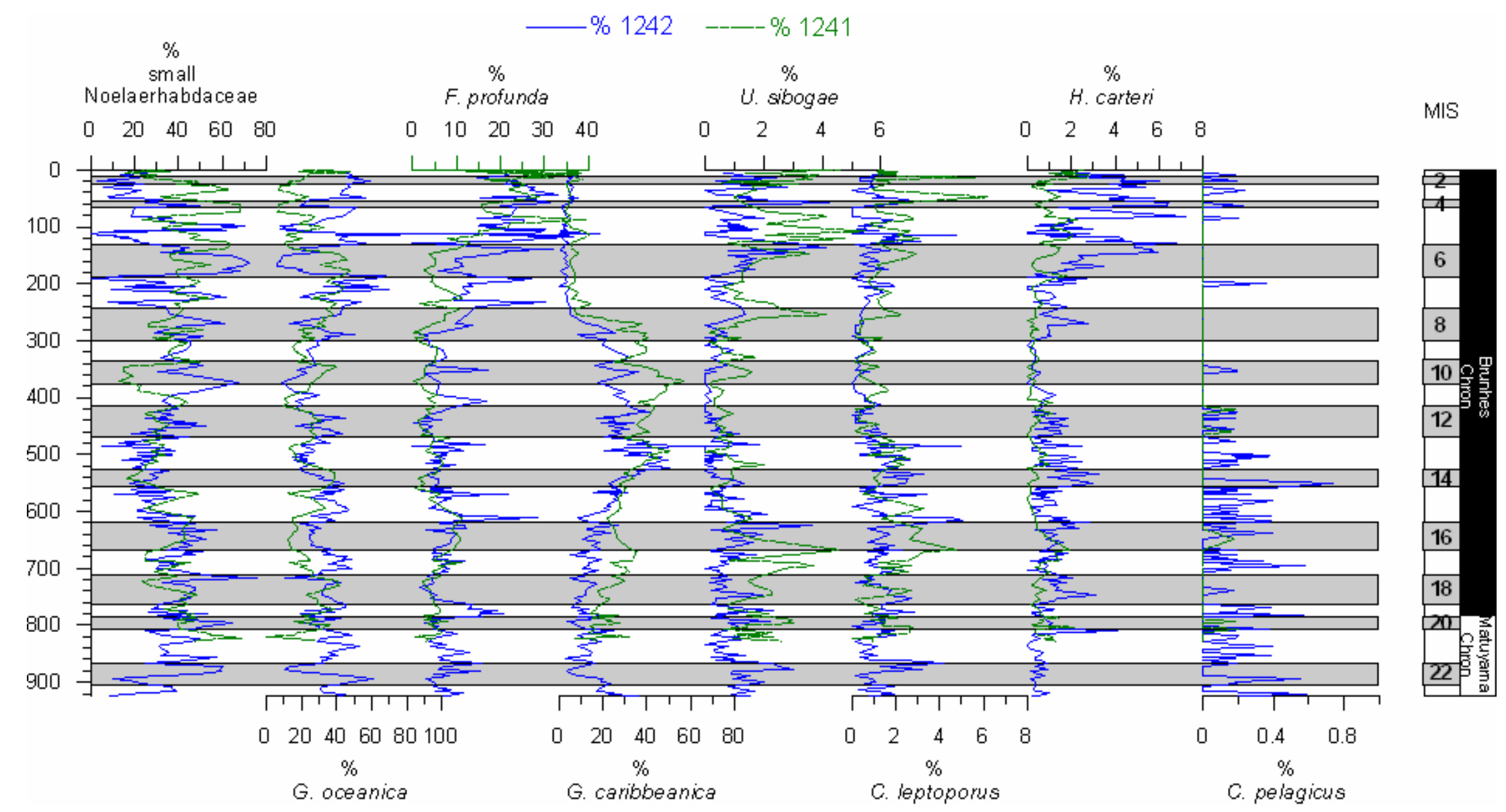

Fig. 6.4. Relative abundances (\%) of the most significant calcareous nannofossil species at sites 1241 and 1242 . Grey bars indicate glacial isotope stages 1 through 22. MIS: Marine Isotope Stage. 


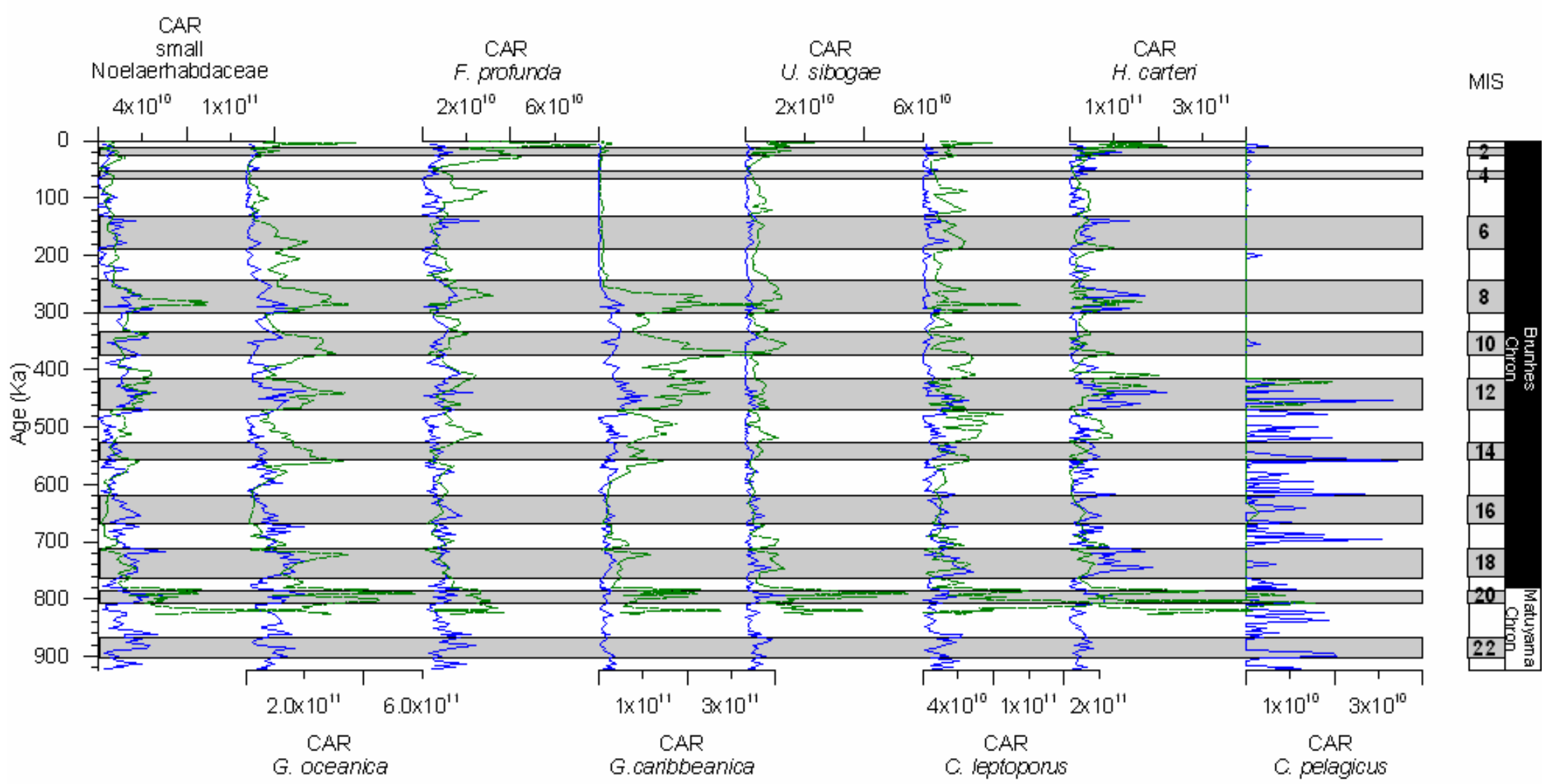

Fig. 6.5. Coccolith-derived carbonate accumulation rates (CAR; $\left.\mathrm{pg}^{\star} \mathrm{Cm}-2^{\star} \mathrm{Ka}-1\right)$ of the most significant calcareous nannofossil species at sites 1241 and 1242 . Grey bars indicate glacial isotope stages 1 through 22. MIS: Marine Isotope Stage. 


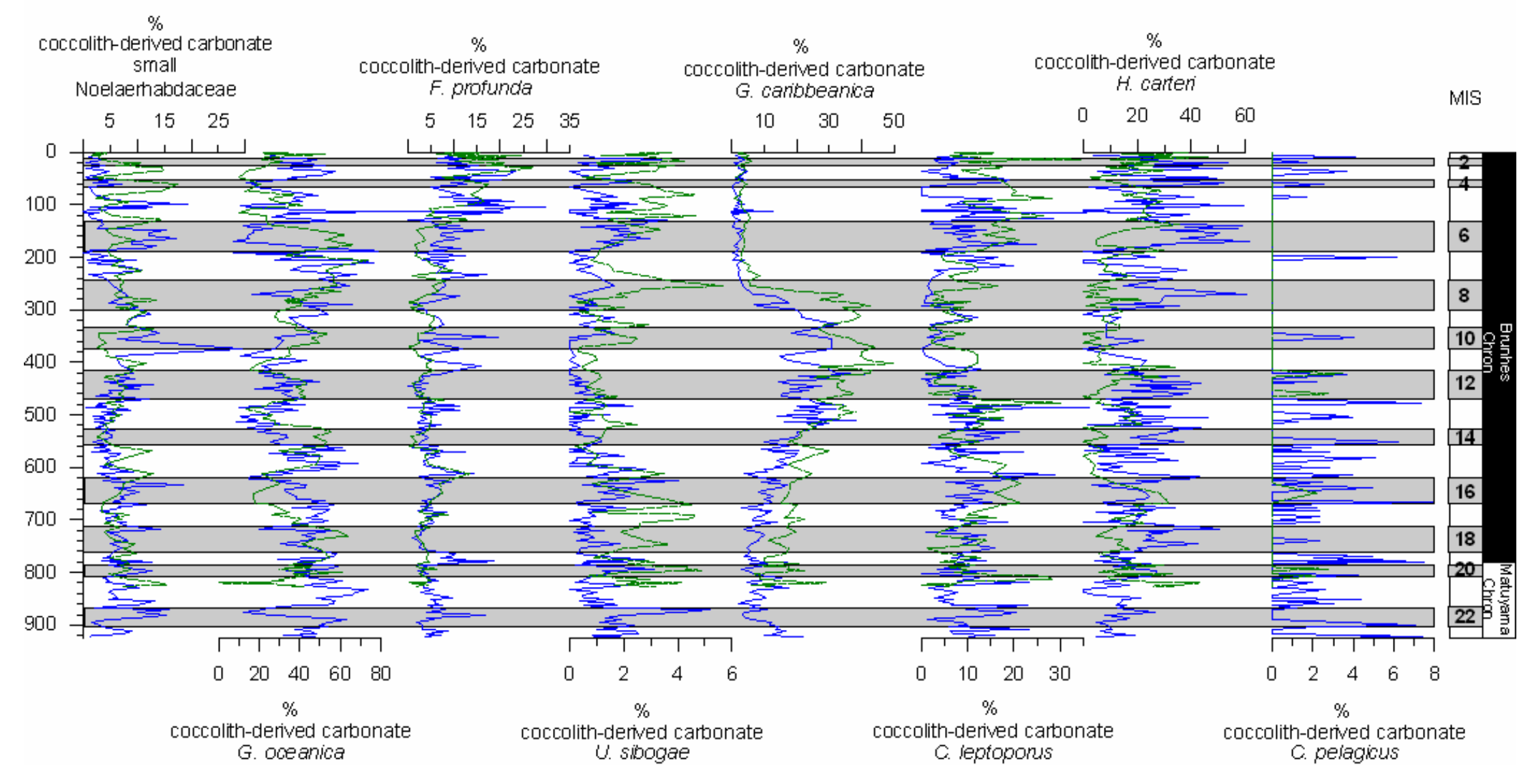

Fig. 6.6. Coccolith-derived carbonate (\%) contributed by the most significant calcareous nannofossil species at sites 1241 and 1242 . Grey bars indicate glacial isotope stages 1 through 22. MIS: Marine Isotope Stage. 
MIS 22 to MIS 6 is the period with the highest $\mathrm{N}$ ratio, total CAR values and species-specific CAR values, showing a maximum during MIS 22 to MIS 8 at Site 1242 (Figs 2, 3). The reconstructed CAR profiles show that $G$. caribbeanica was the most important contributor to the coccolithderived carbonate ( $4 \times 10^{11}$ and $1.2 \times 10^{11} \mathrm{pg}^{*} \mathrm{Ka}^{-1 *} \mathrm{Cm}^{-2}$ at sites 1241 and 1242 respectively) characterising the mid-Brunhes event (MIS 15 to 8 ) at the sites studied. The mid-Brunhes event also shows a clear contribution by G. oceanica species to coccolith-derived carbonate (Fig. 6.5). Another important characteristic of this interval is the low relative abundance of $F$. profunda (below 20\%) with isolated maxima during interglacial periods. During MIS 8 to MIS 6 (283 Ka to $128 \mathrm{Ka}$ ), F. profunda shows a gradual increase in its proportions, giving way to a slow reduction in the $\mathrm{N}$ index at Site 1242. This reduction coincides with the decrease in total and speciesspecific CAR values, and a strong decrease in the abundance of $G$. caribbeanica and in its contribution to coccolith-derived carbonate at both sites (Figs. 6.3, 6.4, 6.5, 6.6).

During MIS 5 to 1, the small Noelaerhabdaceae and G. oceanica still dominate the assemblage. This interval is also characterised by a clear increase in the proportions of $F$. profunda, particularly important during MIS 5, generating a major shift in the $\mathrm{N}$ ratio and CAR values. The reduction in the $\mathrm{N}$ ratio and CAR during MIS 5 also coincides with a deteriorated preservation of the coccolith-derived carbonate at Site 1242 but also with the enhanced preservation pattern observed at Site 1241. The major contribution by F. profunda to the coccolith-derived carbonate has occurred over the past $283 \mathrm{Ka}$, with maxima around $25 \%$, driven by major increases in its abundance (Figs. 6.4, 6.5, 6.6).

\subsection{Disc ussion}

\subsubsection{The $\mathbf{N}$ ratio and coccolith-derived carbonate: presenation or palaeoproductivity profiles?}

The high productivity-carbonate variability in the Eastern Equatorial Pacific (EEP) was first observed by Arrhenius (1952). Its glacial/interglacial pattern was later confirmed by $\delta^{18} 0$ stratigraphy (Emiliani, 1955) and by ${ }^{14} \mathrm{C}$ dating of the carbonates from Arrhenius' research area (Hays et al., 1969). Other authors (Hays et al., 1969, Archer et al., 1991a, b; Karlin et al., 1992; Le and Shackleton, 1992, Lyle et al., 2002, among others) have demonstrated that glacial carbonate maxima are caused by high carbonate production rather than dissolution. However, the possibility of high-productivity carbonate variability has not always been accepted, as stated by 
Berger (1973) and Thompson and Saito (1974), who interpreted that the carbonate profile in the Pacific Ocean reflects preservation rather than a palaeoproductivity pattern.

Variations in carbonate sedimentation have been attributed to variations in the rate of skeletal production by plankton, dissolution, and dilution by non-carbonate content (Dittert et al., 1999). Since changes in the $\mathrm{N}$ ratio and CAR estimations counteract the dilution effect caused by different sedimentary components (biogenic or terrestrial), such proxies are influenced only by production and/or dissolution (Fig. 6.3). First, we assessed whether variations in the $\mathrm{N}$ ratio and CAR profiles might respond to preservation or to a palaeoecological signal. If the $\mathrm{N}$ ratio profile were indeed a preservation record, then palaeoproductivity would only be estimated for glacial events. However, if the $\mathrm{N}$ ratio were a palaeoproductivity signal, it would be expected that calcareous nannofossil production would have been more important than dissolution. Several lines of evidence in the calcareous nannofossil record could elucidate this situation (Figs. 6.2 and 6.3):

Intense dissolution events apply strictly to a few samples during a few interglacial stages, masking the palaeoecological signal at Site 1242; e.g., the end of MIS 15; the middle of MIS 13; the end of MIS 7, and throughout MIS 5 (except MIS 5.3; Fig. 6.3). However, a good to moderate degree of preservation in the rest of the sedimentary sequence at Site 1242 reveals the palaeocological significance, even during the mid-Brunhes event. The mid-Brunhes event was a period when a global increase of carbonate production and severe dissolution occurred (Droxler et al., 2003), although no severe dissolution was identified at sites 1241 and 1242 for that event. The better preservation of the deeper Site 1241 clearly suggests that fluctuations in the $\mathrm{N}$ ratio and in the CAR profiles would have responded to palaeoecological variations in the calcareous assemblage rather than to dissolution in the ETP. The palaeoecological signal is also supported by the enhanced preservation of calcareous nannofossils at Site 1240 over the past $560 \mathrm{Ka}$ (López-Otálvaro et al., 2008a). Site 1240 is situated in the equatorial divergence zone, very close to the modern Galapagos area lysocline (which is located at a depth of $\sim 3000 \mathrm{~m}$; Parker and Berger, 1971). The fact that the sites are located above the lysocline ( 2800 $\mathrm{m}$ in the Panama Basin; Farrell and Prell, 1989) suggests that sediments would not have been strongly affected by dissolution (Luz and Shackleton, 1975).

It could be speculated that dissolution was enhanced in the calcareous nannofossil record when the biogenic carbonate content was lower, although the $\mathrm{N}$ index and the CAR cannot be applied 
to the intervals of poor preservation and barren ones. The reduction in the $\mathrm{N}$ ratio and the CAR values during MIS 13, 7 and 5 at Site 1241 support such an assumption. Furthermore, the higher total CAR values observed reflect the higher carbonate production at Site 1241 than at Site 1242 and hence, account for the differences in preservation at both sites. Adelseck Jr. and Anderson (1978) suggested a decrease in the production of carbonate during MIS 7 and 5 compared to MIS 11 and 9 at a shallow site in the EEP. Furthermore, MIS 5 has been characterised by a lower production of upper photic zone dwellers at other tropical sites in the Pacific and Indian oceans (Beaufort et al., 2001; López-Otálvaro et al., 2008a).

All this evidence points to the idea that the palaeoecological signal was superimposed over the dissolution pattern, reflecting the notion that variations in the CAR paralleled variations in palaeoproductivity. As the CAR increased from low to moderate values, there was a first increase in the production of calcareous nannofossils and their respective tissue carbon. However, even though calcareous nannofossils produced high CAR, remineralization of the organic matter would have delayed the accumulation of carbonate until palaeoproductivity outweighed organic carbon remineralization, causing an increase in the CAR during a period of increases in surface water fertility. According to this model, it may be concluded that the $\mathrm{N}$ ratio and the CAR profiles supported high glacial /low interglacial productivity.

Geochemical evidence has shown that other proxies are directly related to high glacial palaeoproductivity. For example, the alkenone organic proxy was consistently elevated during glacial rather than during interglacial periods throughout the Pleistocene in the ETP (Emeis et al., 1995; Liu and Herbert, 2004); the acummulation rates of barite (BaSO4) have been higher during glacial rather than interglacial events in the Eastern and Central Pacific during the past $450 \mathrm{Ka}$ (BaSO4 is easily preserved in sediments and it is not sensible to burial diagenesis in oxic sediments; Paytan et al., 1996); maximum increases in the bulk of biogenic sedimentary Al/Ti were consistent with increases in biogenic particle fluxes during glacials, reflecting high palaeoproductivity in the Equatorial Central Pacific (ECP) during those events over the past 650 Ka (ECP, Murray et al., 1993); changes in productivity joined fluxes of organic carbon, opal and carbonate, sharing maxima during glacial rather than interglacial periods in three cores located in the ETP at different water depths (the nearby Core VN1-49GC, located at 6032.3N, 92046.2'W, 3580 m water depth; Core V19-28, located at 2028'S, 84039'W, 2670 m water depth; and Core W8402A-14GC, situated at 0057.2'N, 138057.3'W, $4287 \mathrm{~m}$ water depth; Lyle et al., 1988); additional organic carbon measurements in other cores located in the Panama Basin and Peru 
Basin have been interpreted as episodes of increased production during the last glacial maximum (Pedersen, 1983) and glacial stages along the past $600 \mathrm{Ka}$ (Pedersen et al., 1991); phosphorous accumulation rates in the Equatorial Pacific and Southern Ocean show higher glacial values, reflecting increased primary productivity and export production (Filippelli et al., 2007). Additionally, existing dust records indicate higher aerosol levels during the last glacial event, which would have resulted in an enhanced iron supply to surface waters in the equatorial Pacific (Martin, 1990; Prahl, 1991). However, higher Pleistocene values of $\mathrm{NO}_{3}^{-}$(and consequently lower $\delta^{15} \mathrm{~N}$ as the dominant nitrogenous species for new production in the Pacific Ocean) have been observed for glacial events. For the Last Glacial Maximum, these lower values were explained in two records located south of the Panama Basin and one from the Galapagos region, as decreases in nitrate utilization in response to an excess of nitrate supply, over the biological demand (Farrell et al., 1995).

\subsubsection{Glacial/interglacial nutri-themoc line variability}

The high glacial productivity in the euphotic zone, and hence the shallowing of the nutrithermocline in the basin (Figs 6.1, 6.2, 6.3), points to dominant northeast Trade winds that prevented the warm water regime of the NECC in the Panama Basin and displaced the equatorial front southward and generated intensified wind jets in the gulfs of Panama and Papagayo that produced coastal upwelling and located the ITCZ in the southward position. This setting has been suggested by some authors for modern conditions of the northern hemisphere winter (Wyrtki, 1966; McCreary et al., 1989; Chelton et al., 2000a, b; Fiedler, 2002), and a similar association appears to have been important in the ETP on a glacial/interglacial scale. Other authors have demonstrated that Trade wind velocities were markedly enhanced during glacial events, resulting in higher upwelling rates, stronger vertical mixing, and an enhanced downward flux of biogenic sediments (Sarnthein et al., 1988). During interglacial times, the low nutri-thermocline reflected the strengthening of the warm NECC, the northward position of the convergent front between the SEC and the NECC, and the seasonal divergence between the NEC and the NECC at $10^{\circ} \mathrm{N}$ caused by dominant southeast Trade winds that influenced the northernmost position of the ITCZ. The divergence at $10 \mathrm{~N}$ produced an enhancement of the cyclonic circulation of the Costa Rica Dome in the open ocean and generated the upward and lateral displacement of the isotherms associated with it. However, the strengthening of the warm water regime of the NECC depressed -relatively- the nutri-thermocline and reduced productivity in the euphotic zone. Although the sites 
are located in the NECC region, the nutri-thermocline has remained relatively shallow and thus productivity has remained higher over the last $925 \mathrm{Ka}$.

A slight gradient in palaeoproductivity, and thus in the nutri-thermocline deepening, is found between site 1241 and 1242 positions. Our data support a slightly shallower nutri-thermocline at the former site, induced by the eutrophic regime of the EUC. Furthermore, a similar gradient was found between the equatorial divergence zone and the ETP. The coccolith-derived carbonate content at those sites is lower than that observed at the equatorial divergence Site 1240. This is an important result since sites 1241 and 1242 are located outside the more marked eutrophic regime of the ECT (Mix et al., 2003). Hofmann et al. (1981) stated that the thermocline in the divergence thermocline ridge (at $10^{\circ} \mathrm{N}$ ) is as shallow as $10 \mathrm{~m}$. Fiedler (2002, Figs. 1 and 5 ) reported that the thermocline at the equatorial divergence zone is around $40 \mathrm{~m}$ deep, but he did not quote differences in the mean thermocline at our sites, which that author reported as being as shallow as $50 \mathrm{~m}$. The nutri-thermocline in the NECC region is shallower than in other subtropical gyre regions owing to the permanent (although stationary) cyclonic circulation associated with the dome in the region (Holfmann et al., 1981; Fiedler, 2002), the input of nutrients from depth and the lateral supply across the Equatorial Front, and the $10 \mathrm{~N}$ thermocline ridge (Pennington et al., 2006).

\subsubsection{ENSO-like events}

A persistent feature in the palaeoproductivity records is a relatively shallow nutri-thermocline regime over the past $925 \mathrm{Ka}$; it was more apparent during MIS 22 to early MIS 6, and was related to the increase in the wind-driven advection of cool and nutrient-rich water. Productivity was slightly reduced during MIS 8-6, but was still higher and comparable with MIS 22 to 8, suggesting a shallow nutri-thermocline and a La Niña-like state with some oscillations (Figs. 6.1, 6.2 and 6.3). The eutrophic environment recorded during MIS 20 to 6 has been compared to the La Niñalike event by other authors in the Peru upwelling and the equatorial divergence zone (Beaufort et al., 2001; Álvarez, 2005; López-Otálvaro et al., 2008a).

The most striking change in the structure of the upper water column occurred during MIS 5. This resulted in an oligotrophic setting and reveals general sea surface warming, a decrease in sea surface fertility and lower production due to a deepening of the nutri-thermocline (Fig. 6.3). Since the nutri-thermocline in the ETP is subantarctic in origin (Toggweiler et al., 1991; Loubere, 2000; 
Sarmiento et al., 2003; Liu and Herbert, 2004; Loubere et al., 2007; Robinson et al., 2007), a weakening of the Trade wind circulation and a reduction of the subantarctic influence on the ETP would have favored that situation. The oligotrophic environment during MIS 5 has also been characterised in the tropical Pacific and Indian oceans, recording an enhanced abundance of $F$. profunda, low surface palaeoproductivity, and hence a deepening of the nutri-thermocline related to a permanent El Niño-like Event being reported (Beaufort et al., 2001; López-Otálvaro et al., 2008a).

Productivity was lower during MIS 4-1 than that recorded for MIS 22-6, and was also comparable to the productivity reported for MIS 5 . This feature suggests that the weakening of the upwelling in the warm pool was still present during MIS 4-1. In fact, the calcareous nannofossil evidence suggests a general progressive deepening of the nutri-thermocline for the past $283 \mathrm{Ka}$. Liu et al. (2008) observed a gradual increase in the abundances of $F$. profunda and a consequent deepening of the nutri-thermocline in the South China Sea for the past $250 \mathrm{Ka}$. Other authors have suggested a reduction in the Southern Hemisphere circulation over the past $500 \mathrm{Ka}$ (Scharmm, 1985 and Emeis et al., 1995). The extreme eutrophic (MIS 22-6) and oligotrophic (MIS 5) regimes previously indicated by other authors in the Equatorial Pacific Ocean were also recorded in the warm pool in the present work. MIS 4-1 was a period characterised by a less severe eutrophic regime, together with a reduction in the intensity of the Trade winds in the upwelling areas of Peru and the ECT, than those recorded during MIS 22 to 6 , and it has been related to a weaker La Niña-like Event (Beaufort et al., 2001; Álvarez, 2005; López-Otálvaro et al., 2008a). The relatively weaker Southern Hemisphere circulation during MIS 4-1 caused the ETP to be permanently warmer than the equatorial divergence area and therefore induced a deepening of the nutri-thermocline that was more pronounced in the warm pool and that prevented palaeoproductivity in that sector from being recovered as rapidly as the ECT after MIS 5. This evidence allows it to be concluded that the warm pool sector was specifically sensitive to ENSO-like conditions when these events were particularly intense. Sites 1241 and 1242 are not directly under the major upwelling areas (e.g. the equatorial divergence zone and the coastal upwelling of Peru, Mix et al., 2003; Groeneveld, et al., 2006) and so, they provide no direct support for the idea of weaker La Niña-like events during MIS 4-1 in the Eastern EEP. 


\subsubsection{The mid-Brunhes event}

We strongly believe that $G$. caribbeanica represents a robust marker for the mid-Brunhes event during MIS 15 to 8 in the ETP because its higher abundances elicited an important increase in total coccolith-derived carbonate production and hence in enhanced preservation (Figs. 4, 5, 6). The mid-Brunhes event has been closely linked to a global increase in the abundance of $G$. caribbeanica and in total pelagic carbonate production in the Atlantic, Pacific, Indian and Southern oceans (Droxler, 2003; Bollman et al., 1998; Flores et al., 1999; Baumann and Freitag, 2004; Álvarez, 2005; Barker et al., 2006; López-Otálvaro et al., 2008a; Liu et al., 2008). Our calcareous nannofossil records reveal that the mid-Brunhes event was a period related to high productivity and a shallower nutri-thermocline in the ETP, indicating that G. caribbeanica was associated with more fertile water masses. This evidence has also been confirmed by Liu et al. (2008) with the nannofossil $\delta^{13} \mathrm{C}$ record in the South China Sea and with the calcareous nannofossil data of Álvarez, 2005; López-Otálvaro et al., 2008a in the ETP. However, Bollmann et al. (1998) described G. caribbeanica as a taxon that prefers oligotrophic and warm water masses. Other researchers have mentioned an evolutionary overprint to explain its dominance in the assemblage (Bollman et al., 1998; Flores et al., 1999). The present work shows that the midBrunhes event was strongly correlated with the most extreme eutrophic setting in the ETP during the past $925 \mathrm{Ka}$ and was related to a La Niña-like state with some fluctuations.

\subsubsection{Calcareous nannofossil assemblage}

Maximum glacial species-specific CAR peaks of small Noelaerhabdaceae, G. oceanica, $G$. caribbeanica, U. sibogae, C. leptoporus and $H$. carteri suggest their palaeoecological preferences for a eutrophic environment during cooler episodes (Figs. 5, 6). The small Noelaerhabdaceae have been considered as eutrophic indicator in cool waters (Gartner, 1988; Young, 1994; Okada and Wells, 1997; Wells and Okada, 1997; Flores et al., 1999; 2000, 2003; Bollmann et al., 1998; Beaufort et al., 1999; Takahashi and Okada, 2000; Beaufort and Buchet, 2003; López-Otálvaro et al., 2008a). G. oceanica has been shown to have affinities for low-latitude upwelling conditions (Winter, 1982; Mitchell-Innes and Winter, 1987; Hagino et al., 2000; López-Otálvaro et al., 2008a) and for warm, nutrient-rich waters (Roth and Coulbourn, 1982; Gartner, 1988; Houghton and Guptha, 1991; Giraudeau et al., 1993; Kinkel et al., 2000; Cortes et al., 2001; Andruleit and Rogalla, 2002; Boeckel and Baumann, 2004; Hagino and Okada, 2004; Martínez et al., 2005). The connections between U. sibogae (Roth and Berger, 1975; Andruleit et al., 2000; LópezOtálvaro et al., 2008b), H. carteri (Estrada, 1978; Giraudeau, 1992; Pujos, 1992; Flores, 1995) 
and C. leptoporus (Roth and Berger, 1975; Fincham and Winter, 1989; Andruleit and Rogalla, 2002; Flores et al., 2003), with an intensified eutrophic environment, have been confirmed previously by several researchers. The covariance of $C$. leptoporus and H.carteri was also reported by Ziveri et al. (2000) in the Mediterranean Sea.

Our data clearly show that $F$. profunda is a robust marker of the vertical migration of the nutrithermocline in the ETP. The flux of carbonate production in the upper photic zone outweighed carbonate production in the lower photic zone even when palaeoproductivity diminished, as revealed by the $F$. profunda-specific CAR. This evidence reinforces the idea that palaeoproductivity was relatively high in the warm pool during the past $925 \mathrm{Ka}$. In addition, the persistent individual CAR values of $F$. profunda indicate that the nutrient availability in the lower photic zone did not change significantly, as it did in the upper photic zone, and that the variability in the accumulation of the upper photic zone taxa would be related to fluctuations in the regime of the sea surface water. F. profunda has been reported as a deep ocean-dwelling (around 100 and $220 \mathrm{~m}$ ) species in tropical and subtropical oceans that prefers low light and a high nutrient content (Okada and Honjo, 1973; Okada and Mclntyre, 1977; among others), and this suggests changes in the nutri-thermocline and sea surface palaeoproductivity (Molfino and Mclntyre, 1990a, b; Castradori, 1993; Young, 1994; Jordan et al., 1996; Okada and Wells, 1997; Wells and Okada, 1997; Beaufort et al., 1997, 1999, 2001; Ziveri and Thunell, 2000; Beaufort and Buchet, 2003; Flores et al., 2000; Liu and Herbert, 2004; Baumann and Freitag, 2004; López-Otálvaro et al., 2008a, b; Marino et al., 2008; Liu et al., 2008).

The maximum abundances and the most continuous record of $C$. pelagicus were recorded during $925 \mathrm{Ka}$ to $430 \mathrm{Ka}$, during the period of more fertile water massess; however, the subordinate character of $C$. pelagicus suggests that this taxon is outside its palaeoecological affinity area. The palaeoecological preferences of $C$. pelagicus for cooler and nutrient-rich waters have been addressed in several other studies carried out in the North Atlantic (Cachão and Moita, 2000; Parente et al., 2004; Narciso et al., 2006).

\subsection{Conclusions}

A comparative study of calcareous nannofossil preservation between sites 1241 and 1242 reveals good to moderate preservation at those sites, supporting the palaeoecological 
significance of the calcareous nannofossil records used here; i.e. they reflect the notion that the palaeoecological signal is the primary pattern, and that dissolution effects are enhanced when palaeoproductivity is reduced.

The glacial/interglacial variability in the CAR record is closely related to the $\mathrm{N}$ ratio, and hence the CAR record was used here as a palaeoproductivity proxy.

Variations in palaeoproductivity at sites 1241 and 1242 are primarily associated with the variability in the strengthening of the NECC but also with its associated hydrographic and atmospheric features at long-term scales. High glacial productivity reflects a shallowing of the nutri-thermocline related to a weakening of the warm water regime of the NECC, southern displacement of the Equatorial Front, intensification of the wind-jets driving upwellings and the lateral displacement of the isotherms, dominant northeast Trade winds, and southern migration of the ITCZ. Low interglacial productivity reflects a deepening of the nutri-thermocline in response to a piling up of the warmer eastward waters in the ETP dragged by an intensified NECC and the northward position of the convergent Equatorial Front, caused by dominant southeast Trade winds and the northward location of the ITCZ.

A Persistent feature throughout the last $925 \mathrm{Ka}$ is the highest productivity and the shallowest nutri-thermocline during MIS 22 to early MIS 6. This setting suggests a strengthening in the winddriven advection of cool and nutrient-rich water, indicating a period related to a La Niña-like state. A more oligotrophic environment is suggested for MIS 5 by the increase in the abundance of $F$. profunda and a drop in palaeoproductivity as a consequence of a deeper nutri-thermocline. This evidence is indicative of an El Niño-like state. During MIS 4-1 palaeoproductivity was not significantly different from that observed for MIS 5 . This setting suggests that the cross-equatorial wind and upwelling were still less intense during MIS 4-1 than during MIS 22 to 6 . This implies that the warm pool was not recovered from the disturbance during MIS 5 as was the ECT. Our records are not indicative of a prominent La Niña-like state during MIS 4-1, as previously reported for the upwelling regions of the EEP.

The dominant role played by $G$. caribbeanica serves as an exceptional marker of the midBrunhes event during MIS 15 to 8 owing to its higher production, which controlled the stronger accumulation of coccolith-derived carbonate in the ETP. The dominance of this species is thus 
recorded as coinciding with an extreme high-productivity environment, indicating that its high occurrence was very coherent with a La Niña-like Event in the ETP.

Glacial CAR increases in the small Noelaerhabdaceae, G. oceanica, G. caribbeanica, U. sibogae, $C$. leptoporus and $H$. carteri reveal their preference for an eutrophic environment in the warm pool over the past $925 \mathrm{Ka}$. The frequent occurrence of $C$. pelagicus prior to the past $500 \mathrm{Ka}$ suggests a more marked palaeoproductivity pattern in the ETP. The lower values of the $F$. profundaspecific CAR indicate of a relatively lower carbonate production in the lower photic zone as compared to that seen for the shallow photic zone, reflecting a shallowed nutri-thermocline that would have favored a relatively higher palaeoproductivity regime in the ETP over the past $925 \mathrm{Ka}$. The relative steady $F$. profunda-specific CAR values additionally reflect the notion that the nutrient supply to the lower photic zone was relatively steady compared with that occurring in the upper photic zone, indicating that variations in the CAR values of the upper photic zone taxa are related to fluctuations in the upper photic zone palaeoenvironmental regime.

\section{Aknowledgements}

The authors wish to thank the ODP Program Leg 202 for providing samples for calcareous nannofossil analysis. We are also very grateful to Dr. Maria Angela Bassetti and an anonymous reviewer for their careful revision and contributions which have improved this paper considerably. Financial support from the Spanish Ministerio de Educación y Ciencia, through an FPI Fellowship (BES-2003-0010) provided to G.E. López-Otálvaro and from Spanish projects BTE2002-04670 and REN2003-08642-C02/CLI from the Ministerio de Educación y Ciencia, and SA088/04 from the Consejería de Educación de la Junta de Castilla y León is acknowledged.

\section{References}

Adelseck Jr., C.G., Anderson, T.F. 1978. The late Pleistocene record of productivity fluctuaions in the eastern equatorial Pacific Ocean. Geology 6, 388-391.

Alvarez, M.C. 2005. Reconstrucción paleoceanográfica del margen continental del Pacífico durante los últimos 800 Ka mediante el análisis de asociaciones de cocolitóforos. Ph. D Thesis, Universidad de Salamanca, Salamanca, Spain, $151 \mathrm{pp}$.

Andruleit, H., Rogalla, U. 2002. Coccolithophores in surface sediments of the Arabian Sea in relation to environmental gradients in surface waters. Marine Geology 3132, 1-22.

Archer, D.E., 1991a. Equatorial Pacific calcite preservation cycles: production or dissolution?. Paleoceanography 6, 561-571.

Archer, D.E. 1991b. Modelling the calcite lysocline. Journal of Gephysical Research 96, 17037-17050.

Archer, D., Aiken, J., Balch, W., Barber, D., Dunne, J., Flament, P., Gardner, W., Garside, C., Goyet, C., Johnson, E., Kirchman, D., McPhaden, M., Newton, J., Peltzer, E., Welling, L., White, J., Yoder, 
J. 1997. A meeting place of great ocean currents: shipboard observations of a convergent front at ${ }^{\circ} \mathrm{N}$ in the Pacific. Deep-Sea Research II 44, 1827-1849.

Arrhenius, G. 1952. Properties of the sediment and their distribution: Sediment cores from the East Pacific. Swedish Deep-Sea Expedition 1947-1948, 5, 1-228.

Barber, R.T., Chávez, F.P. 1983. Biological consequences of El Niño. Science 222, 1203-1210.

Barber, R.T., Chavez, F.P. 1986. Ocean variability in relation to living resources during the 1982/83 El Niño. Nature 319, 279-285.

Barker, S., Archer, D., Booth, L., Elderfield, H., Henderiks, J., Rickaby, R. 2006. Globally increased pelagic carbonate production during the Mid-Brunhes dissolution interval and the $\mathrm{CO}_{2}$ paradox of MIS 11 . Quaternary Science Reviews 25, 3278-3293.

Baumann, K.-H., Freitag, T. 2004. Pleistocene fluctuations in the northern Benguela Current system as revealed by coccolith assemblages. Marine Micropaleontology 52, 195-215.

Baumgartner, T.R., Christensen, N., Jr. 1985. Coupling of the Gulf of California to large-scale interannual variability. Journal of Marine Research 43, 825-848.

Beaufort, L., Buchet, N. 2003. Variability of specific coccolith $\mathrm{CaCO}_{3}$ weight, and primary production in the western Pacific warm pool during the last glacial cycle. Geophysical Research Abstracts (European Geosciences Union) 5, 13225.

Beaufort, L., Lancelot, Y., Camberlin, P., Cayre, O., Vincent, E., Bassinot, F., Labeyrie, L. 1997. Insolation cycles as a major control of equatorial Indian Ocean primary production. Science 278, 1451-1454

Beaufort, L., Bassinot, F.C., Vincent, E. 1999. Primary production response to orbitally induced variations of the Southern Oscillation in the Equatorial Indian Ocean. In: Abrantes, F., Mix, A.C. (Eds.), Reconstructing Ocean History: a Window into the Future. Kluwer Academic/Plenum, New York, 245-272

Beaufort, L., de Garidel-Thoron, T., Mix, A.C., Pisias, N.G. 2001. ENSO-like forcing on oceanic primary production during the Late Pleistocene. Science 293, 2440-2444.

Benway, H., Mix, A. 2004. Oxygen isotopes, upper-ocean salinity, and precipitation sources in the eastern tropical Pacific. Earth and Planetary Science Letters 224, 493-507.

Benway, H.M., Mix, A.C., Haley, B.A., Klinkhammer, G.P. 2006. Eastern Pacific Warm Pool paleosalinity and climate variability: 0-30 kyr. Paleoceanography 21, 2006. doi: 10.1029/2005PA001208.

Berger, W.H. 1968. Planktonic foraminifera: Selective and paleoclimatic interpretations: Deep-Sea Research 15, 31-43.

Berger, W.H. 1973. Deep-Sea carbonates: Pleistocene dissolution cycles. Journal of Foraminiferal Research 3, 187-195.

Bjerknes, I. 1969. Atmospheric teleconnection from the equatorial Pacific. Monthly Weather Review 97, 163-172.

Böeckel, B., Baumann, K.-H. 2004. Distribution of coccoliths in surface sediments of the south-eastern South Atlantic Ocean: ecology, preservation and carbonate contribution. Marine Micropaleontology 51, 301-320.

Bollmann, J., Baumann, K.-H., Thierstein, H. 1998. Global dominance of Gephyrocapsa coccoliths in the late Pleistocene: selective dissolution, evolution or global environmental change? Paleoceanography $13,517-529$.

Cachão, M., Moita, M.T. 2000. Coccolithus pelagicus, a productivity Proxy related to moderate fronts off Western Iberia. Marine Micropaleontology 39, 131-155.

Castradori, D. 1993. Calcareous nannofossils and the origin of eastern Mediterranean sapropel. Paleoceanography 8, 459-471.

Chelton, D.B., Freilich, M.H., Esbensen, S.K. 2000a. Satellite observations of the wind jets off the Pacific coast of Central America. Part I: Case studies and statistical characteristics. Monthly Weather Review 128, 1993-2018.

Chelton, D.B., Freilich, M.H., Esbensen, S.K. 2000b. Satellite observations of the wind jets off the Pacific coast of Central America. Part II: Regional relationships and dynamical considerations. Monthly Weather Review 128, 2019-2043.

Clement, A.C., Seager, R., Cane, M.A. 1999. Orbital controls on the El Niño/Southern Oscillation and the tropical climate. Paleoceanography 14, 441-456.

Clement, A.C., Seager, R., Cane, M.A. 2000. Suppression of El Niño during the mid-Holocene by changes in the Earths's orbit. Paleoceanography 15, 731-737. 
Cortes, M.Y., Bollmann, J., Thierstein, H. 2001. Coccolithophore ecology at the HOT station ALOHA Hawaii. Deep-Sea Research II 48, 1957-1981.

Dessier, A., Donguy, J.R. 1987. Response to El Niño signals of the epiplanktonic copepod populations in the eastern tropical Pacific. Journal of Geophysical Research 92 (C13), 14393-14403.

Dittert, N., Baumann, K.-H., Bickert, R., Henrich, R., Huber, R., Kinkel, H., Meggers, H. 1999. Carbonate dissolution in the deep-sea: methods, quantification and paleoceanographic application. In: Fischer, G., Wefer, G. (Eds.), Use of Proxies in Paleoceanography: Examples from the South Atlantic. Springer, Berlin, Heidelberg, pp. 255-284.,

Droxler, A.W., Alley, R.B., Howard, W.R., Poore, R.Z., Burckle, L.H. 2003. Unique and exceptionally long interglacial marine isotope stage 11: window into Earth warm future climate. In: Droxler, A.W., Poore, R.Z., Burckle, L.H. (Eds.), Earth's Climate and Orbital Eccentricity. The Marine Isotope Stage 11 Question. . Monograph Series, vol. 137. AGU, Washington, DC, pp. 1-14.

Emeis, K.-C., Doose, H., Mix, A., Schulz-Bull, D. 1995. In: Pisias, N.G., Mayer, L. A., Janecek, T.R., Palmer-Julson, A., van Andel, T.H. (Eds.), Alkenone Sea Surface Temperatures and Carbon Burial at Site 846 (Eastern Equatorial Pacific Ocean): the Last 1.3 M.y. Proc. ODP, Sci. Results, vol. 138. Ocean Drilling Program, College Station, TX, pp. 605-613.

Emiliani, C. 1955. Pleistocene temperature. Journal of Geology 63, 538-578.

Estrada, M. 1978. Mesoscale heterogeneities of the phytoplankton distribution. In: Boje, R., Tomezak, M. (Eds.), The Upwelling Region of northWest Africa. (Upwelling Ecosystems). Springer, Berlin, pp. 15-23.

Farrell, J.W., Prell, W. 1989. Climatic change and $\mathrm{CaCO}_{3}$ preservation: $\mathrm{An} 800,000$ year bathymetric reconstruction from the Central Equatorial Pacific Ocean. Paleoceanography 4, 447-466.

Farrell, J.W., Prell, W. 1991. Pacific CaCO3 Preservation and $\delta^{180}$ Since 4 Ma: Paleoceanic and Paleoclimatic Implications. Paleoceanography 6, 485-498.

Farrell, J.W., Pedersen, T.F., Calvert, S.E., Nielsen, B. 1995. Glacial-interglacial changes in nutrient utilization in the equatoria Pacific Ocean. Nature 377, 514-517.

Fiedler, P.C. 2002. The annual cycle and biological effects of the Costa Rica Dome. Deep-Sea Research I 49, 321-338.

Fiedler, P.C., Talley, L.D. 2006. Hydrography of the eastern tropical Pacific: A review. Progress in Oceanography $69,143-180$.

Filippelli, G.M., Latimer, J., Murray, R.W., Flores, J.A. 2007. Productivity records from the Southern ocean and the equatorial Pacific Ocean: Testing the glacial Shelf-Nutrient Hypothesis. Deep-Sea Research II 54, 2443-2452.

Fincham, M.J., Winter, A. 1989. Paleoceanographic interpretations of coccoliths and oxygen-isotopes from sediments from the surface of the Southwest Indian Ocean. Marine Micropaleontology 13, 325351.

Flores, J.A., Sierro, F.J., Raffi, I. 1995. Evolution of the calcareous nannofossil assemblage as a response to the paleoceanographic change in the Eastern Equatorial Pacific from 4 to $2 \mathrm{Ma}$ (leg 138, Site 849 and 852). Proc. ODP, Initial Rep. 138, 163-176.

Flores, J.A., Sierro, F.J. 1997. Revised technique for calculation of calcareous nannofossil accumulation rates. Micropaleontology 43, 321-324.

Flores, J.A., Gersonde, R., Sierro, F.J. 1999. Pleistocene fluctuations in the Agulhas current retroflection based on the calcareous plankton record. Marine Micropaleontology 37, 1-22.

Flores, J.A., Bárcena, M.A., Sierro, F.J. 2000. Ocean-surface and wind dynamics in the Atlantic Ocean off Northwest Africa during the last 140000 years. Palaeogeography, Palaeoclimatology, Palaeoecology 161, 459-478.

Flores, J.A., Marino, M., Sierro, F.J., Hodell, D.A., Charles, C.D. 2003. Calcareous plankton dissolution pattern and coccolithophore assemblages during the last 600 kyr at ODP Site 1089 (Cape Basin, South Atlantic): paleoceanographic implications. Palaeogeography, Palaeoclimatology, Palaeoecology 196, 409-426

Flores, J.-A., Wei, W., López-Otálvaro, G.E., Alvarez, C., Sierro, F.J. 2006. In: Tiedemann, R., Mix, A.C., Richter, C., Ruddiman, W.F. (Eds.), Data Report: Tropical and Equatorial Calcareous Nannofossil Pleistocene Biostratigraphy, ODP Leg 202. Proc. ODP, Sci. Results, vol. 202. College Station, TX, Ocean Drilling Program, pp. 1-10. doi:10.2973/odp.proc.sr.202.214.2006.

Gartner, S. 1988. Paleoceanography of the Mid-Pleistocene. Marine Micropaleontology 13, 23-46. 
Geitzenauer. K.R., Roche, M.B., Mclntyre, A. 1977. Coccolithophore biogeography of the North Atlantic and Pacific surface sediments. In: Ramsay (Ed.). Oceanic Micropaleontology. Academic Press, New York, pp. 973-1008.

Gersonde, R., Hodell, D.A., Blum, P., et al., 1999. Proc. ODP, Init. Repts., 177. Ocean Drilling Program , College Station, TX. doi:10.2973/odp.proc.ir.177.1999.

Giraudeau, J. 1992. Distribution of Recent nannofossils beneath the Benguela system: Southwest African continental margin. Marine Geology 108, 219-237.

Giraudeau, J., Monteiro, P.M.S., Nikodemus, K. 1993. Distribution and malformation of living coccolithophores in the northern Benguela upwelling system of Namibia. Marine Micropaleontology 22, 93-110.

Groeneveld, J., Steph, S., Tiedemann, R., Garbe-Schönberg, D., Sturm, A. 2006. Pliocene mixed-layer oceanography for Site 1241 , using combined $\mathrm{Mg} / \mathrm{Ca}$ and $\delta^{18}$ Oanalyses of Globigerinoides sacculifer. In: Tiedemann, R., Mix, A.C., Richter, C., Ruddiman, W.F. (Eds.), Data Report: Tropical and Equatorial Calcareous Nannofossil Pleistocene Biostratigraphy, ODP Leg 202. Proc. ODP, Sci. Results, vol. 202. College Station, TX, Ocean Drilling Program, pp. 1-10. doi:10.2973/odp.proc.sr.202.209.2006.

Hagino, K., Okada, H. 2004. Floral response of coccolithophores to progressive oligotrophication in the South Equatorial Current, Pacific Ocean. In: Shiyomi, M., Kawahata, H., Koizumi, H., Tsuda, A., Awaya, Y. (Eds.), Global Environmental Change in the Ocean and on Land. TERRAPUB, pp. 121-132.

Hagino, K., Okada, H., Matsuoka, H. 2000. Spatial dynamics of coccolithophore assemblages in the Equatorial western-central Pacific Ocean. Marine Micropaleontology 39, 53-72.

Hays, J.D., Saito, T., Opdyke, N.D., Burckle, L.H. 1969. Pliocene-Pleistocene sediments of the equatorial Pacific: Their paleomagnetic, biostratigraphic, and climatic record. Geological Society of America Bulletin 8, 1481-1514.

Hiramatsu, C., De Deckker, P. 1997. The calcareous nannoplankton assemblages of surface sediments in the Tasman and Coral seas. Palaeogeography, Palaeoclimatology, Palaeocology 131, 257-285.

Hofman, E., Busalacchi, A.J., Q'Brien J. 1981. Wind generation of the Costa Rica Dome. Science 214, 552-554. doi: $10.1126 /$ science. 214.4520 .552

Houghton, S.D., Guptha, M.V.S. 1991. Monsoonal and fertility controls on recent marginal sea and continental shelf coccolith assemblages from the western Pacific and northern Indian oceans. Marine Geology 97, 251-259.

Hofmann, E.E., Busalacchi, A.J., Q'Brien, J.J. 1981. Wind generation of the Costa Rica Dome. Science $214,552-554$.

Jordan, R.W., Zhao, M., Eglinnton, G., Weaver, P.P.E. 1996. Coccolith and alkenone stratigraphy and paleoceanography at an upwelling site off NW Africa (ODP 658C) during the last 130,000 years. In: Moguilevsky, A., Whatley, R. (Eds.), Microfossils and Oceanic Environments. Aberystwyth Press, University of Wales, pp. 111-130.

Karlin, R., Lyle, M., Zahn, R. 1992. Carbonate variations in the Northeast Pacific during the late Quaternary. Paleoceanography 7, 43-61.

Kessler, W.S. 2002. Mean three-dimensional circulation in the northeast tropical Pacific: Journal of Physical Oceanography 32, 2457-2471.

Kessler, W.S. 2006. The circulation of the eastern tropical Pacific: a review. Progress in Oceanography $69,181-217$.

Kinkel, H., Baumann, K.-H., Cepek, M. 2000. Coccolithophores in the equatorial Atlantic Ocean: response to seasonal and Late Quaternary surface water variability, Marine Micropaleontology 39, 87-112.

Knappertsbusch, M., Cortes, M.Y., Thierstein, H.R. 1997. Morphologic variability of the coccolithophorid Calcidiscus leptoporus in the plankton, surface sediments and from the Early Pleistocene. Marine Micropaleontology 30, 293-317.

Koutavas, A., Lynch-Stieglitz, J. 2003. Interglacial dynamics of the eastern equatorial Pacific cold tongueIntertropical Convergence Zone system reconstructed from oxygen isotope records. Paleoceanography 18, doi:10.1029/2003PA000894.

Le, J., Shackleton, N.J. 1992. Carbonate dissolution fluctuations in the Western Equatorial Pacific during the late Quaternary. Paleoceanography 7, 21-42. 
Leduc, G., Vidal, L., Tachikawa, K., Rostek, F., Sonzogni, C., Beaufort, L., Bard, E. 2007. Moisture transport across Central America as a positive feedback on abrupt climatic changes. Nature 445, 908-911. doi: 10.1038/nature05578.

Liu, Z., Herbert, T.D. 2004. High-latitude influence on the eastern equatorial Pacific climate in the early Pleistocene epoch. Nature 427, 720-723.

Liu, C., Wang, P., Tian, J., Cheng, X. 2008. Coccolith evidence for Quaternary nutricline variations in the southern South China Sea. Marine Micropaleontology, doi:10.1016/j.marmicro.2007.11.008.

Loubere, P. 2000. Marine control of biologial production in the eastern equatorial Pacific Ocean. Nature 406, 497-500.

Loubere, P., Samantha, A., Siedlecki, L., Bradtmiller, L.I. 2007. Organic carbon and carbonate fluxes: Links to climate change. Deep-Sea Research II 54, 437-446.

López-Otálvaro, G.-E., Flores, J.A., Sierro, F.J., Cacho, I. 2008a. Variations in coccolithophorid production inthe Eastern Equatorial Pacific at ODP Site 1240 over the last seven glacial-interglacial cycles. Marine Micropaleontology, doi: 10.1016/j.marmicro.2007.11.009.

López-Otálvaro, G.-E., Flores, J.A., Sierro, F.J., Cacho, I., Grimalt, J.O., Michel, E., Cortijo, E., Labeyrie, L. 2008b. Late Pleistocene paleoproductivity patterns during the last climatic cycle in the Guyana Basin: the calcareous nannoplankton clue. E-Earth. Accepted.

Luz, B., Shackleton, N.J. 1975. $\mathrm{CaCO}_{3}$ solution in the tropical East Pacific during the past 130,000 years. Cushman Foundation for Foraminiferal Research Special Publication 13, 142-150.

Lyle, M., Murray, D.W. 1988. The record of Late Pleistocene biogenic sedimentation in the Eastern Tropical Pacific Ocean. Paleoceanography 3, 39-59.

Lyle, M., Mix, A., Pisias, N. 2002. Patterns of $\mathrm{CaCO}_{3}$ deposition in the eastern tropical Pacific Ocean for the last $150 \mathrm{kyr}$ : Evidence for a southeast Pacific depositional spike during marine isotope stage (MIS) 2. Paleoceanography 17, doi: 10.1029/2000PA000538.

Marino, M., Maiorano, P., Lirer, F. 2008. Changes in calcareous nannofossil assemblages during the midPleistocene Revolution. Marine Micropaleontology, doi:10.1016/j.marmicro.2007.11.010.

Martin, J.H. 1990. Glacial-interglacial $\mathrm{CO}_{2}$ change: the iron hypothesis. Paleoceanography 5, 1-13.

Martínez, I., Rincon, D., Yokoyama, Y., Barrows, T. 2005. Foraminifera and coccolithophorid assemblage changes in the Panama Basin during the last deglaciation: Response to sea-surface productivity induced by a transient climate change. Palaeogeography, Palaeoclimatology, Palaeoecology 234, 114-126.

McCreary, Jr. J.P., Lee, H.S., Enfield, D.B. 1989. The response of the coastal ocean to strong offshore winds: With application to circulations in the Gulfs of Tehuantepec and Papagayo. Journal of Marine Research 47, 81-109.

Mitchell-Innes, B.A., Winter, A. 1987. Coccolithophores: a major phytoplankton component in mature upwelled waters off the Cape Peninsula, South Africa in March, 1983. Marine Biology 95, 25-30.

Mix, A.C., Tiedemann, R., Blum, P., et al., 2003. Proc. ODP, Init. Repts., 202. Ocean Drilling Program, College Station, TX. doi:10.2973/odp.proc.ir.202.2003.

Molfino, B., Mclntyre, A. 1990a. Nutricline variation in the equatorial Atlantic coincident with the Younger Dryas. Paleoceanography 5, 997-1008.

Molfino, B., Mclntyre, A. 1990b. Precessional forcing of nutricline dynamics in the equatorial Atlantic. Science 249, 766-769.

Murray, R.W., Leinen, M., Isern, A.R. 1993. Biogenic flux of Al to sediment in the Central Equatorial Pacific Ocean: Evidence for increased productivity during glacial periods. Paleoceanography 8, 651-670.

Narciso, A., Cachão, M., de Abreu, L. 2006. Coccolithus pelagicus subsp. pelagicus versus Coccolithus pelagicus subsp. braarudii (Coccolithophore, Haptophyta): A proxy for surface subartic Atlantic waters off lberia during the last 200 kyr. Marine Micropaleontology 59, 15-34.

Okada, H., Honjo, S. 1973. The distribution of oceanic coccolithophorids in the Pacific. Deep-Sea Research 20, 355-374.

Okada, H., Honjo, S. 1975. Distribution of coccolithophores in marginal seas along the western Pacific ocean and in the Red Sea. Marine Biology, 31(3):271-286.

Okada, H., Mclntyre, A., 1977. Modern coccolithophores of the Pacific and North Atlantic Oceans. Micropaleontology 23, 1-55. 
Okada, H., Wells, P. 1997. Late Quaternary nannofossil indicators of climate change in two deep-sea cores associated with the Leewin Current off Western Australia. Palaeogeography, Palaeoclimatology, Palaeoecology 131, 413-432.

Pak, H., Zaneveld, J.R. 1974. Equatorial Front in the Eastern Pacific Ocean. Journal of Physical Oceanography 4, 570-578.

Parente, A., Cachão, M., Baumann, K.-H., de Abreu, L., Ferreira, J. 2004. Morphometry of Coccolithus pelagicus s.l. (Coccolithophore, Haptophyta) from offshore Portugal, during the last $200 \mathrm{kyr}$. Micropaleontology 50 (supplement), 107-120.

Parker, F.L., Berger, W.H. 1971. Faunal and solution patterns of planktonic foraminifera in surface sediments of the South Pacific. Deep-Sea Research 18, 73-107.

Paytan, A., Kastner, M., Chavez, F.P. 1996. Glacial to interglacial fluctuations in productivity in the Equatorial Pacific as indicated by marine barite. Science 274, 1355-1357.

Pedersen, T.F. 1983. Increased productivity in the eastern equatorial Pacific during the last glacial maximum (19,000 to 14,000 yr B.P.). Geology 11, 16-19.

Pedersen, T.F., Nielsen, B., Pickering, M. 1991. Timing of Late Quaternary productivity pulses in the Panama Basin and implications for atmospheric $\mathrm{CO}_{2}$. Paleoceanography 6, 657-677.

Pena, L., Cacho, I., Ferreti, P., Hall, M.A. 2008. ENSO-like variability during glacial Terminations and interlatitudinal teleconnections. Paleoceanography, doi:10.1029/2008PA001620, in press.

Pennington, J.T., Mahoney, K.L., Kuwahara, V.S., Kolber, D.D., Calienes, R., Chavez, F.P. 2006. Primary production in the eastern tropical Pacific: a review. Progress in Oceanography 69, 283-317.

Philander, S.G.H. 1990. El Niño, La Niña and the Southern Oscillation. Academic Press, San Diego, CA, $289 \mathrm{pp}$.

Philander, G. 1995. El Niño and La Niña. In: Pirie, R.G. (Ed.), Oceanography: Contemporary Readings in Ocean Sciences, Oxford Univ. Press, Oxford, U.K., 72-87.

Philander, S.G.H., Gu, D., Halpern, D., Lambert, G., Lau, N.-C., Li, T., Pacanowski, R.C. 1996. Why the ITCZ is mostly north of the Equator. Journal of Climate 9, 2958-2972.

Prahl, F.G. 1991. Prospective use of molecular paleontology to test for iron limitation on marine primary productivity. Marine Chemistry 39, 167-185.

Pujos-Lamy, A. 1977. Emiliania et Gephyrocapsa (Nannoplancton calcaire): Biométrie et intéret biostratigraphique dans le Pleistocène supérieur marin des Açores. Revista Española de Micropaleontología 9, 69-84.

Pujos, A. 1992. Calcareous nannofossils of Plio-Pleistocene sediments from the northwestern margin of tropical Africa. In Summerhayesm C.P., Prell, W.L., Emeis, K.C. (Eds.). Upwelling systems: Evolution since the early Miocene. Geological Society Special Publication, London, 64, 343-359.

Raffi, I. 2002. Revision of the early-middle Pleistocene calcareous nannofossil biochronology (1.75-0.85 Ma). Mar. Micropaleontol. 45, 25-55.

Raffi, I., Flores, J.A. 1991. Pleistocene through Miocene calcareous nannofossils from Eastern Equatorial Pacific Ocean (Leg 138). Proc. ODP, Sci. Results, vol. 138. Ocean Drilling Program, College Station, TX, pp. 233-286.

Raffi, I., Backman, J., Rio, D., Shackleton, N.J. 1993. Early Pleistocene and late Pliocene nannofossil biostratigraphy and calibration to oxygen isotope stratigraphies from DSDP Site 607 and ODP Site 677. Paleoceanography 8, 387-404.

Rickaby, R.E.M., Bard, E., Sonzogni, C., Rostek, F., Beaufort, L., Barker, S., Rees, G., Schrag, D.P. 2007. Coccolith chemistry reveals secular variations in the global ocean carbon cycle? Earth and Planetary Science Letters 253, 83-95.

Robinson, R.S., Mix, A., and Martinez, P. 2007. Southern Ocean control on the extent of denitrification in the southeast Pacific over the last 70 ka. Quaternary Science Reviews 26, 201-212. doi:10.1016/j.quascirev.2006.08.005

Rodríguez-Rubio, E., Schneider, W., Abarca del Río, R. 2003. On the seasonal circulation within the Panama Bight derived from satellite observations of wind, altimetry and sea surface temperature. Geophysical Research Letters 30, 1410, doi: 1029/2002GL016794.

Rost, B., Riebesell, U. 2004. Coccolithophore calcification and the biological pump: response to environmental changes. In: Thierstein, H.R., Young, Y.R. (Eds.), Coccolithophores - From Molecular Processes to Global Impact. Springer-Verlag, Berlin, pp. 99-125. 
Roth, P.H., Berger, W.H. 1975. Distribution and dissolution of coccoliths in the South and Central Pacific. Dissolution of Deep-sea Carbonates. Cushman Foundation for Foraminiferal Research, Special Publication 13, 87-113.

Roth, P.H., Coulbourn, W.T. 1982. Floral and solution patterns of coccoliths in surface sediments of the North Pacific. Marine Micropaleontology 7, 1-52.

Sánchez, G., Calienes, R., Zuta, S. 2000. The 1997-1998 El Niño and its effects on the coastal marine ecosystem off Peru. California Cooperative Fisheries Investigations Reports 41, 62-86.

Sarmiento, J.L., Gruber, N., Brzezinski, M.A., Dunne, J.P. 2003. High latitude controls of thermocline nutrients and low latitude biological productivity. Nature 427, 56-60.

Sarnthein, M., Winn, K., Duplessy, J-C., Fonrugne, M.R. 1988. Global variations of surface ocean productivity in low and mid latitudes: influence on $\mathrm{CO}_{2}$ reservoirs of the deep ocean and atmosphere during the last 21,000 years. Paleoceanography 3, 361-399.

Scharmm, C.T. 1985. Implications of radiolarian assemblages for the Late Quaternary Paleoceanography of the eastern equatorial Pacific. Quaternary Research 25, 204-218.

Shackleton, N.J., Berger, A., Peltier, W.R. 1990. An alternative astronomical calibration of the Pleistocene timescale based on ODP Site 677. Transactions of the Royal Society of Edinburgh: Earth Sciences 81, 251-261.

Smith, W.H.F., Sandwell, D.T. 1997. Global sea floor topography from satellite altimetry and ship depth soundings. Science 277, 1956-1962.

Steinmetz, J.C. 1994. Sedimentation of coccolithophores. In: Winter, A., Siesser, W.G. (Eds.). Coccolithophores, Cambridge University Press, Cambridge, pp. 179-197.

Takahashi, K., Okada, H. 2000. The paleoceanography for the last 30, 000 years in the southeastern Indian Ocean by means of calcareous nannofossils. Marine Micropaleontology 40, 83-103.

Thierstein, H.R., Geitzenauer, K.R., Molfino, B., Shackleton, N.J. 1977. Global synchroneity of late Quaternary coccolith datum levels: validation by oxygen isotopes. Geology 5, 400-404.

Thompson, P.R., Saito, T. 1974. Pacific Pleistocene sediments: Planktonic foraminifera dissolution cycles and geochronology. Geology 2, 333-335.

Toggweiler, J., Dixon, K., Broecker, W. 1991. The Peru upwelling and the ventilation of the South Pacific thermocline. Journal of Geophysical Research 96(C11), 20467-20497.

Turk, D., Lewis, M.R., Harrison, W. G., Kawano, T., Asanuma, I. 2001a. Geographical distribution of new production in the western/central equatorial Pacific during El Niño and non-El Niño conditions. Journal of Geophysical Research 106(C3), 4501-4515.

Turk, D., McPhaden, M. J., Busalacchi, A., Lewis, M. R. 2001b. Remotely sensed biological production in the equatorial Pacific. Science 293, 471-474.

Turney, C.S., Kershaw, A.P., Clemens, S.C., Branch, N., Moss, P.T., Fifield, L.K. 2004. Millenial and orbital variations of El Niño/Southern Oscillation and high-latitude climate in the last glacial period. Nature 428, 306-310.

Wang, C., Fiedler, P.C. 2006. ENSO variability in the eastern tropical Pacific: a review. Progress in Oceanography 69, 239-266.

Weaver, P.P.E ., Thomson, J. 1993. Calculating erosion by deep-sea turbidity currents during initiation and flow. Nature $364,136-138$.

Wei, W. 1993. Calibration of upper Pliocene-lower Pleistocene nannofossil events with oxygen isotope stratigraphy. Paleoceanography 8, 85-89.

Wells, P., Okada, H. 1997. Response of nannoplankton to major changes in sea-surface temperature and movements of hydrological fronts over Site DSDP 594 (south Chatham Rise, southeastern New Zealand), during the last $130 \mathrm{kyr}$. Marine Micropaleontology 32, 341-363.

Winter, A., 1982. Paleoenvironmental interpretation of Quaternary coccolith assemblages from the Gulf of Aquaba (Elat), Red Sea. Revista Española de Micropaleontología 14, 291-314.

Wyrtki, K. 1964. Upwelling in the Costa Rica Dome. Fisheri Bulletin 63, 355-372.

Wyrtki, K. 1966. Oceanography of the Eastern Equatorial Pacific Ocean. Oceanography and Marine Biology Annual Review 4, 33-68.

Wyrtki, K. 1967. Circulation and water masses in the eastern equatorial Pacific Ocean. International Journal of Oceanology and Limnology 1, 117-147.

Young, J.R. 1994. Functions of coccoliths. In: Winter, A., Siesser, W.G. (Eds.). Coccolithophores, Cambridge University Press, Cambridge, pp. 63-82. 
Young, J.R., Ziveri, P. 2000. Calculation of coccolith volume and its use in calibration of carbonate flux estimates. Deep-Sea Research A 47, 1679-1700.

Zaucker, F., Broecker, W.S. 1992. The influence of atmospheric moisture transport on the fresh water balance of the Atlantic drainage basin: general circulation model simulations and observations, Journal of Geophysical Research 97, 2765- 2773.

Zentara, S., Kamykowski, D. 1977. Latitudinal relationships among temperature and selected plant nutrients along the west coast of North and South America. Journal of Marine Research 35, 321337.

Ziveri, P., Thunell, R.C. 2000. Coccolithophores export production in Guaymas Basin, gulf of California: response to climatic forcing. Deep-Sea Research II 47, 2073-2100. 


\title{
Late Pleistocene paleoproductivity pattems during the last climatic cycle in the Guyana Basin: the calcareous nannoplankton clue
}

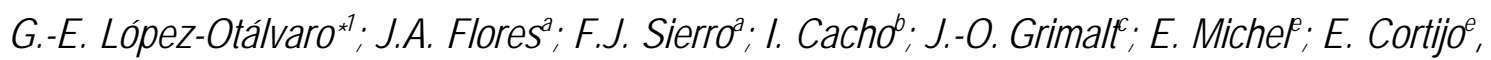 \\ L. Labeyrie \\ ${ }^{a}$ Department of Geology. University of Salamanca. Plaza de la Merced s/n. E-37008 Salamanca, \\ Spain. \\ ${ }^{b}$ Department of Stratigraphy, Paleontology and Marine Geosciences, University of Barcelona, \\ Martí i Franquès, E-08028 Barcelona, Spain \\ 'Department of Environmental Chemistry, Institute of Chemical and Environmental Research \\ (IIQAB-CSIC), Jordi Girona 18, E-08034 Barcelona, Spain \\ 'Laboratoire des Sciences du Climat et de l'Environnement, IPSL, Laboratoire Mixte, CEA-CNRS- \\ UVSQ, Parc du CNRS, F-91198 Gif-sur-Yvette Cedex, France
}

Published in e-Earth, "in press"

Abstract

http://www.electronic-earth-discuss.net/3/11/2008/eed-3-11-2008.html

Key words

7.1. Introduction and Background

7.2. Oceanographic setting

7.3. Materials and methods

7.3.1. Core location and materials

7.3.2. Age model and biostratigraphy

7.3.3. Calcareous nannoplankton prepartion and counting techniques

7.3.4. Taxonomic information

7.4. Preservation

7.5. Results

7.5.1. Variations in the assemblage

7.5.2. Variations in the $\mathrm{N}$ ratio and total NAR profiles

7.6. Discussion

7.6.1. Productivity of calcareous nannoplankton: the end of MIS 6 to MIS 5

7.6.2. Productivity of calcareous nannoplankton: MIS 4 to the early Holocene

7.6.3. The nutri-thermocline dynamic and the Northern Hemisphere connection

7.6.4. River runoff

7.6.5. Dissolution of calcareous nannoplankton

7.7. Conclusions

Aknowledgements

References

*Correspondence to: G.-E. López-Otálvaro (gatsbyemperatriz@usal.es) 


\section{Abstract}

Variations in the assemblage and abundance of calcareous nannoplankton have revealed changes in oceanic and atmospheric dynamics in the Guyana Basin over the last climatic cycle, mainly linked to variations in the southeast Trade winds. Records of the palaeoproductivity index of coccolithophores ( $\mathrm{N}$ ratio) allowed us to monitor nutri-thermocline fluctuations. Nannofossil accumulation rates (NAR) vary closely with the $\mathrm{N}$ ratio, indicating a strong correlation between these two palaeoproductivity proxies. Lower palaeoproductivity during glacial substages of the last climatic cycle indicates a deep nutri-thermocline (deep stratification of the mixed layer) as a consequence of the piling up of warm water dragged by the NEC. This setting was favored by the southern shift of the ITCZ and Trade winds that blew onshore in the Guyana Basin. On the contrary, intervals of higher palaeoproductivity during interglacial substages of the last climatic cycle, suggest a shoaling of the nutri-thermocline promoted by the developement of the NECC that dragged the warm water regime piled up in the Guyana Basin by the NEC. This scenario is favored by the northern displacement of the Intertropical Convergence Zone (ITCZ) that causes the southeast Trade winds blew alongshore. Additionally, higher peaks of palaeoproductivity occur during interglacial substages of MIS 6-5 compared with those ocurred during interglacial substages of MIS 4-2 and the early Holocene, indicating more eutrophic conditions and a more northern position of the ITCZ during the former interglacials. Palaeoproductivity and the nutrithermocline dynamics follow the insolation curve at high northern latitude $(65 \circ \mathrm{N})$, suggesting a Northern Hemisphere latitudinal connection and thus indicating a link between the Northern Hemisphere insolation, the ITCZ and the Trade wind dynamics during the last climatic cycle.

Key words: Calcareous nannoplankton, palaeoproductivity, late Pleistocene, Guyana Basin, Western Tropical Atlantic, Guyana Current, Amazon River.

\subsection{Introduction and Background}

Coccolithophorids are photosynthetic planktonic algae living in the photic layer of the ocean (Hibberd, 1976, Brand, 1994; Young, 1994; Winter et al., 1994). They play a significant role in carbonate precipitation (Siesser and Winter, 1994; Steinmetz, 1994). The temporal and spatial distribution of coccolithophores may respond to major environmental factors in the upper oceanic layer such as temperature, nutrients, the trophic regime and sunlight levels (Brand, 1994; Young, 1994). For example, F. profunda, a phytoplankton species, thrives in the lower photic layer (Okada and Honjo, 1973; Okada and McIntyre, 1977) at times of high nutrient concentration, 
pointing to a deep nutricline (e.g., Molfino and Mclntyre, 1990a, b; de Menocal, 1995; Beaufort et al., 1997; Wells and Okada, 1997; Flores et al., 1999; 2000; Kinkel et al., 2000; Beaufort et al., 2001; Gibbs et al., 2004). The relationship between dwellers from the upper photic zone and those from the lower photic zone has been used to monitor nutri-thermocline depth in the tropical Atlantic (Molfino and Mclntyre, 1990a, b), in the Western Tropical Atlantic (Kinkel et al., 2000; Bassinot et al., 1997), as well as in the other parts of the ocean (Okada and Honjo, 1973; Young, 1994; Okada and Wells, 1997; Wells and Okada, 1997; Beaufort et al., 1997, 1999, 2001, 2003; Beaufort and Buchet, 2003; Flores et al., 2000; Liu and Herbert, 2004; Baumann and Freitag, 2004; López-Otálvaro et al., 2008).

The production and sedimentation of coccolith-derived calcite in the deep ocean plays an important role in the carbon cycle through photosynthesis and calcification (Westbroek et al., 1993; Marsh, 2003; Brand, 1994, Young, 1994; Steinmetz, 1994). These organisms are one of the most important contributors to carbonate sedimentation in the open ocean and in continental slopes (Milliman, 1993; Steinmetz, 1994), such as in the Guyana Basin (Shipboard Scientific Party, 2003), an area controlled by a setting of hemipelagic deposition. The Guyana Basin is located in the western side of the Tropical Atlantic Ocean. This region has been documented as the main route of interhemispheric heat flux exchange from the South to the North Atlantic.

Several published contributions have documented the significance of the calcareous nannofossil assemblages and other micropalaeontological groups as proxies of surface water mass dynamic and palaeoproductivity in the Western Tropical Atlantic (WTA). Molfino and McIntyre (1990a, b) proposed a model whereby the nutri-thermocline topography is related to the Trade wind dynamic in the equatorial Atlantic. They used the relative abundances of $F$. profunda species, with higher proportions of this species associated with a deep nutri-thermocline and lower proportions a shallow nutri-thermocline. The downcore records of coccolithophores from the Western Tropical Atlantic from Kinkel et al (2000) indicated a high interglacial palaeoproductivity and a shallow thermocline compared to glacial stages and substages during the last climatic cycle. Bassinot et al. (1997) used comparative records of carbonate accumulation rates and the relative abundance of $F$. profunda from Kinkel et al. (2000) to interpret that a nutri-thermocline displacement drove palaeoproductivity and carbonate concentration, with the thermocline displacement responding to the close relationship between Trade wind and the Intertropical Convergence Zone (ITCZ) dynamics from $930 \mathrm{Ka}$ to $685 \mathrm{Ka}$ in the Ceara Rise. Also in the Ceara Rise, Rühlemann et al. (2001) reconstructed the thermocline depth from planktonic foraminifer isotope gradients, and 
compared this to the terrigenous sediment content and the relative abundance of $F$. profunda (Kinkel et al., 2000); they suggested low accumulation rates during periods of shallower thermocline indicate a higher primary production during interglacial substages of MIS 5 . Vink et al. (2001) presented new evidence of interlatitudinal teleconnections between Tropical and North Atlantic Ocean during the past $58 \mathrm{Ka}$ using high resolution calcareous dinoflagellate cyst and organic carbon records. The authors found that high accumulation rates of calcareous dinoflagellate cysts occur slightly before the northern Atlantic Heinrich Events indicating that shifts in the zonality of the low-latitude winds would be partly responsible for the climatic variability related with those events. Vink et al. (2002) show high interglacial accumulation rates of Thoracosphaera heimii and calcareous dinocysts in the Western and Eastern Tropical Atlantic when the proportions of $F$. profunda were reduced, the nutri-thermocline was shallowed and the nutrient supply was higher over the past $150 \mathrm{Ka}$.

Our work focuses on a high-resolution micropalaeontological analysis of calcareous nannoplankton over the last climatic cycle (the last $155 \mathrm{Ka}$ ). The primary goal is to reconstruct the patterns of calcareous nannoplankton production and their relationship with fluctuations in the nutri-thermocline and ocean dynamics in the Guyana Basin (Fig. 7.1).

\subsection{Oceanographic setting}

The Guyana Basin is located in the area of annual shift of the Intertropical Convergence Zone (ITCZ) that moves between $10^{\circ} \mathrm{N}$ and $5 \circ \mathrm{S}$ (Müller-Karger, 1989). The northward or southward position of the ITCZ causes a significant impact on water discharge from the Amazon and Orinoco rivers, as well as variations in the direction and speed of the Guyana Current (GC) and the southeast Trade winds (Müller-Karger, 1989; Wilson et al., 2002; Ffield, 2005; Stramma et al., 2005) (Fig. 7.1).

During the boreal summer, the southeast Trade winds are stronger and, the South Equatorial Current (SEC) and the North Brazilian Undercurrent (NBUC, which brings salty water from the South Atlantic) are well developed (da Silveira et al., 1994; Stramma et al., 1995 and Masson and Delecluse, 2000). During that season, the westward displacement of the SEC pileup surface waters along the eastern coast of South America deepening the nutri-thermocline in the Western Tropical Atlantic (WTA) (Hastenrath and Merle, 1987). When the SEC reaches South America, it 
splits into two branches: one turns southward, supplying the Brazilian current (BC), and the other one takes off towards the north and feeds the North Brazilian Current (NBC), which is called the Guyana Current (GC) when it reaches the Guyana Basin (Fig. 7.1).

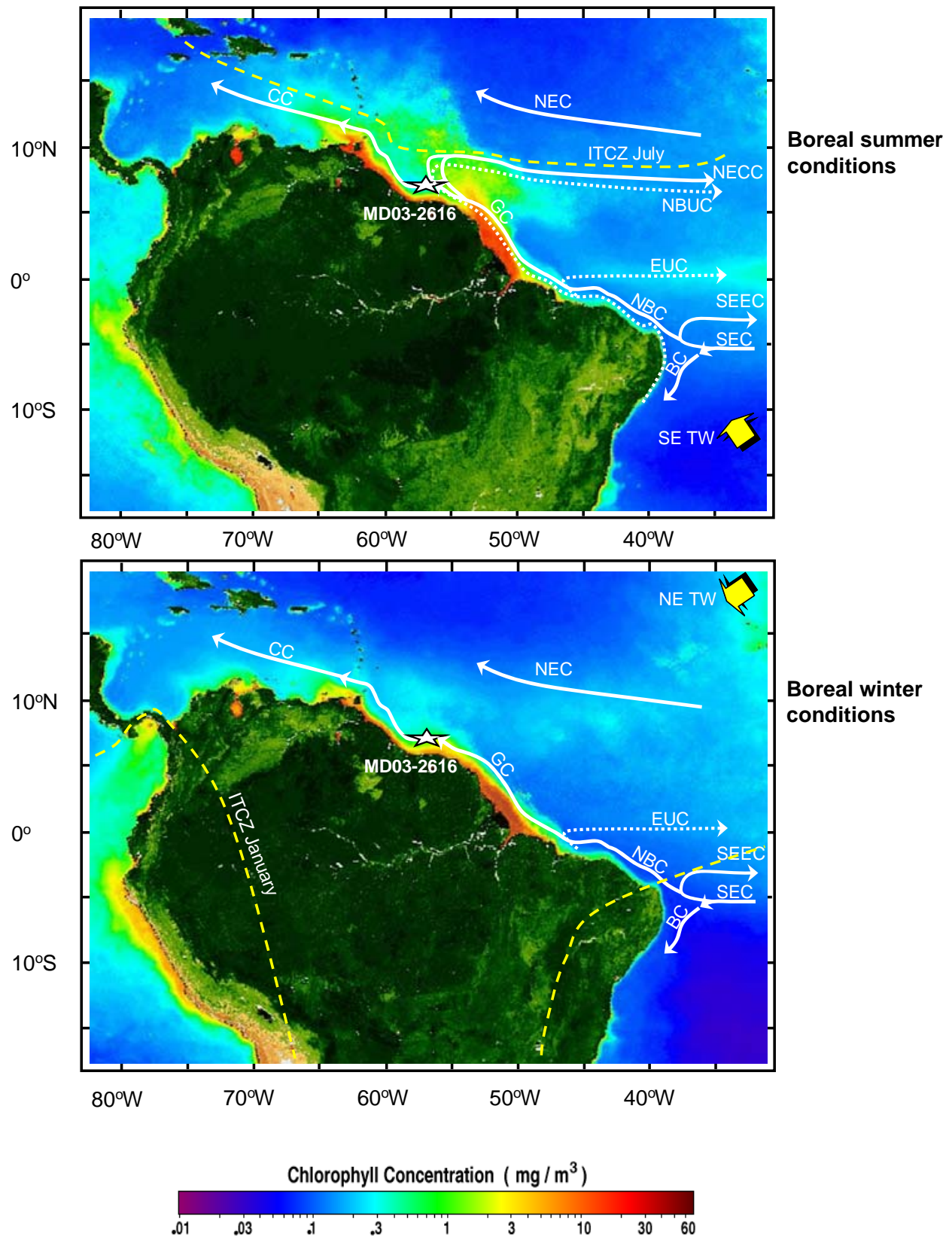

Fig. 7.1. Satellite images acquired by the SEAWIFS Program at the location of the Core MD03-2616. Hydrographic and atmospheric features in WTA: Surface currents are indicated by continuous lines and subsurface currents are indicated by dotted lines. SEC: South Equatorial Current, BC: Brazilian Current, NBC: North Brazilian Current, CC: Caribbean Current, NECC: North Equatorial Counter Current, SECC: South Equatorial Counter Current, EUC: Equatorial Undercurrent, NBCU: North Brazilian Countercurrent, ITCZ: Intertropical Convergence Zone. (The hydrographic features were adopted from Richardson et al., 1994 and Haug et al., 2003. The image background was taken from http://oceancolor.gsfc.nasa.gov/SeaWiFS).

The Intertropical Convergence Zone (ITCZ) is displaced to the northernmost position $\left(6^{\circ} \mathrm{N}-10^{\circ} \mathrm{N}\right)$, generating a wet season north of $\sim 5^{\circ} \mathrm{N}$ (Müller-Karger and Aparicio-Castro, 1994). The NBC (or 
GC) is mostly retroflexed, contributing to the North Equatorial Countercurrent (NECC) between $5 \circ \mathrm{N}-10 \circ \mathrm{N}$ (Müller-Karger et al., 1988), allowing anticyclonic rings to enter the continental margin and pass northwestward to merge with the Orinoco River plume (Müller-Karger, 1989; Ffield, 2005). These eddies transport and advect the Amazon River plume and, together with the NBUC, develop a zone of minimum surface salinity, warm temperatures and a shallower and stronger halocline, where the river flow is dispersed (Masson and Delecluse, 2000). The other component of the GC is directed to the North Atlantic, transporting relatively warm and fresher waters via the Caribbean Sea (feeding Caribbean current, CC) and the Gulf of Mexico (where it supports the Gulf Stream, GS) (Showers and Bevis, 1988; Schmitz, 1995; Stramma and Schott, 1996; LynchStieglitz et al., 1999).

During the boreal winter the northeast Trade winds dominate and push the ITCZ to the southermost position ( $\left.0^{\circ}-5 \circ S\right)$, causing dry conditions in the north of South America. River plumes decrease in intensity, and together with the GC, are mostly driven into the coastal area, carrying relatively colder and saltier waters to the North Atlantic. During this season, the NBUC mainly feeds the Equatorial Undercurrent (EUC) at the equator and cannot cross the equator to the North Atlantic (Masson and Delecluse, 2000 and references therein). This atmosphere-ocean dynamic in the WTA plays a major role in the cross-equatorial heat flux to the North Atlantic (Ffield, 2005), producing relatively cooler surface waters south of the equator, and warmer surface waters north of the equator $\left(>24^{\circ} \mathrm{C}\right)$ in the WTA.

\subsection{Materials and Methods}

\subsubsection{Core location and materials}

Core MD03-2616 was recovered in the Guyana Basin in the Western Tropical Atlantic (WTA) $\left(7048.75^{\prime} \mathrm{N}, 53000.80^{\prime} \mathrm{W}\right)$ at a water depth of $1233 \mathrm{~m}$, during the PICASSO Cruise by the Marion Dufresne R/N (Fig. 7.1). This area is characterized by the influence of the Guyana current at the continental margin of French Guyana. The core shows an undisturbed high-quality sedimentary section of $39 \mathrm{~m}$ spanning the Pleistocene. It consists mainly of olive green silt and clay, rich in foraminifera and calcareous nannoplankton with slight bioturbation levels and high organic matter remains (Shipboard Scientific Party, 2003). Our focus here is on the uppermost $18 \mathrm{~m}$ (a detailed description was reported by the Shipboard Scientific Party, 2003). 


\subsubsection{Age model and biostratigraphy}

The age-depth assignments are primarily based on 16 oxygen isotope control points determined on tests of the benthonic foraminifer Uvigerina peregrina. Stable isotope analyses were performed using an automated carbonate preparation line coupled to a Finningan MAT 251 mass spectrometer at the Laboratoire des Sciences du Climat et de l'Environnement in Gif-sur-Yvette (France). Isotopic events were identified up to Marine Isotope Stage 6, MIS 6 ( $\sim 155 \mathrm{Ka})$, by comparison of the benthic $\delta^{180}$ from the Core MD03-2616 (Unpublished data) and the benthic $\delta^{180}$ stack of Lisiecki and Raymo (2005) (Fig. 7.2).

We analyzed the $>150 \mu \mathrm{m}$ fraction to detect the qualitative abundance of planktonic foraminifer species typical of the WTA. Qualitative study of planktonic foraminifer biostratigraphy allowed the identification of the Ericson Climatic Biozones W1, X, Y, Z of Ericson and Wollin (1956) and the $Y_{\text {P.oblia }}$ of Kennett and Huddlestun (1972). In our record, Biozone W1 was identified by the consistent and lower occurrence of the Globorotalia menardii group from the bottom (155 Ka) of the section studied up to $133 \mathrm{Ka}$. This event was dated by Kenneth and Huddlestun (1972) from $150 \mathrm{Ka}$ to Termination II (128 Ka); i.e., slightly above ours. Biozone X was dated from $133 \mathrm{Ka}$ to the middle phase of substage 5.1 (84.3 Ka). Prell and Damuth (1978) reported the top of the Biozone $X$ at $85 \mathrm{Ka}$; i.e. slightly below ours. The $G$. menardii complex is absent from Biozone $Y$ (Ericson and Wollin, 1956; Kennett and Huddlestun, 1972), as we report here. The disappearance datum of Pulleniatina obliquiloculata $\left(Y_{P \text {. obliq }}\right)$ was recognized at $\sim 39 \mathrm{Ka}$, but this datum is diachronous across the tropical Atlantic (Prell and Damuth, 1978; Kennett and Huddlestun, 1972). Biozone $Z$ was identified close to the end of MIS 2 (over the latest Wisconsin) in agreement with Kennett and Huddlestun (1972), although other authors have placed its bottom at the MIS 1/2 boundary (Ericson and Wollin, 1956; Maslin and Mikkelsen, 1997) (Fig. 7.2).

According to currently available data, the reversal in the abundance of the coccolithophore species E. huxleyi and Gephyrocapsa muellerae is not clear. Thierstein et al. (1977) placed this datum at around 73-85 $\mathrm{Ka}$. The absence of Pseudoemiliania lacunosa, the relatively low abundance of $G$. caribbeanica species (less than $3 \%$ ), and the occurrence of both $E$. huxleyi and G. muellerae along the interval studied suggest that our sediment sequence is younger than 268 Ka (Fig. 7.2). 


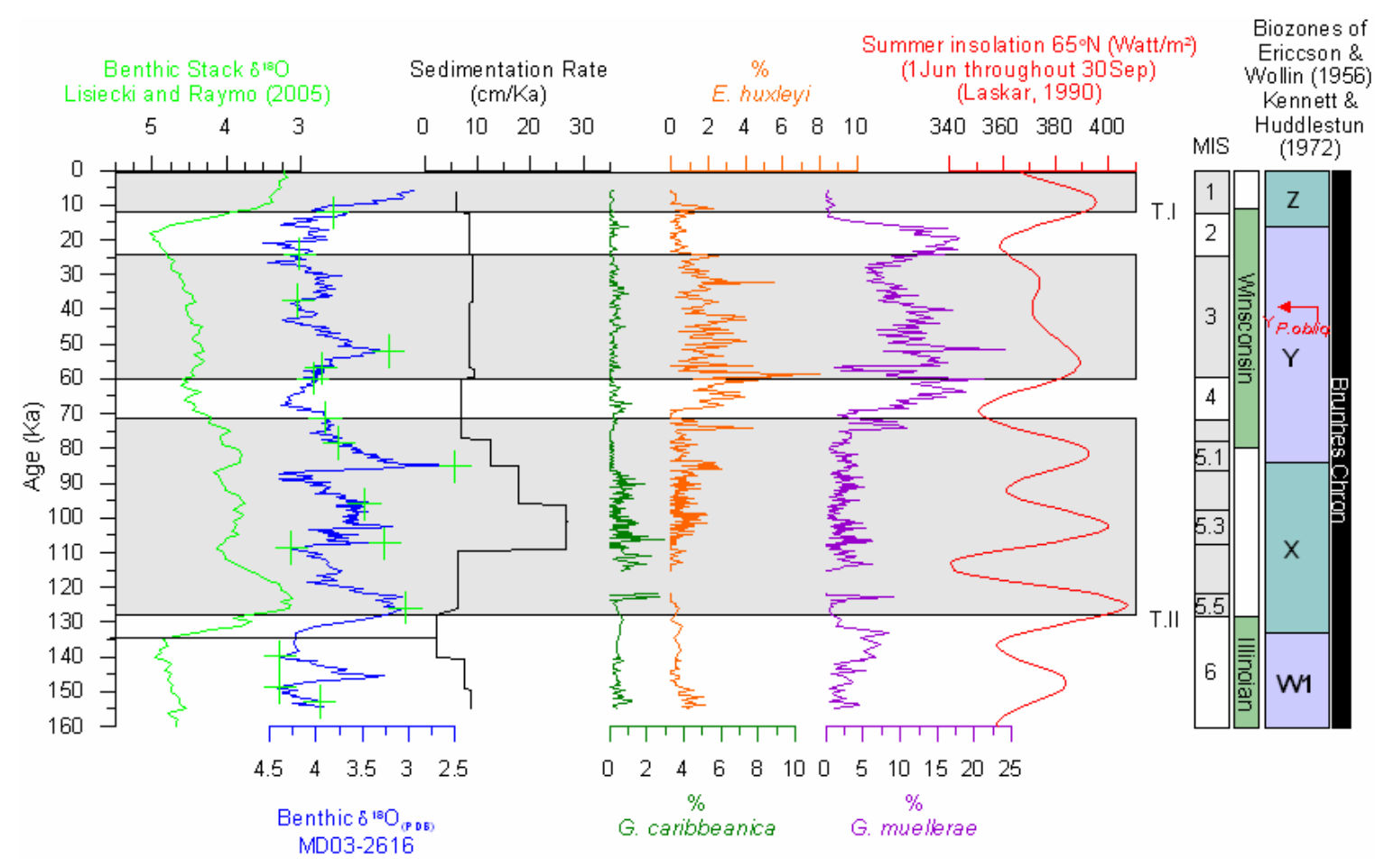

Fig. 7.2. Benthic $\delta^{180}$ stack record (Lisiecki and Raymo, 2005), benthic $\delta^{180}$ record from Core MD03-2616 (Unpublished data), sedimentation rates at Core MD03-2616 and the relative abundance of the specific index species from calcareous nannofossils at Core MD03-2616. The biozones $X, Y, Z$ and $W$ represent the biostratigraphic events from planktonic foraminifers identified at Core MD03-2616. The $Y_{\text {P.oblia }}$ biozone corresponds to the disappearance of Pulleniatina obliquiloculata. The green crosses in the $\delta^{18} O$ record from Core MD03-2616 correspond to the control points used for constructing the chronostratigraphic framework at Core MD03-2616. Grey bars indicate interglacial marine isotope stages 1 throughout 5. MIS 5.1, 5.2, 5.3, 5.4, 5.5 correspond to the substages of MIS 5. Wisconsin and Illinoian episodes correspond to the Ice Ages between $12 \mathrm{Ka}$ and $80 \mathrm{Ka}$ in the former, and $128 \mathrm{Ka}$ and $310 \mathrm{Ka}$ in the later. T.I=Termination I, T.II= Termination II. MIS: Marine Isotope Stage.

\subsubsection{Calcareous nannoplakton preparation and counting techniques}

Slides were prepared following the decantation technique outlined by Flores and Sierro (1997) to obtain quantitative measurements (coccoliths per gram, accumulation rates of coccoliths and coccolith-derived carbonate). These slides were analysed at a 4-cm spacing (representing approximately $\sim 0.120 \mathrm{Ka}$ to $\sim 0.700 \mathrm{Ka}$ ) with a polarized microscope at $1000 \mathrm{X}$ magnification. 380 samples were examined, and more than 500 coccoliths were counted on each slide in order to identify the coccolith assemblage quantitatively.

We estimated variations in the $\mathrm{N}$ ratio (modified from Flores et al., 2000; López-Otálvaro et al., 2008) which is a function based on the relative proportion of taxa usually living in the upper photic zone (small Noelaerhabdaceae, E. huxleyi and G. oceanica; e.g., Okada and Honjo, 1973; Okada and McIntyre, 1979; Giraudeau, 1992; Young, 1994; Okada and Wells, 1997; Wells and Okada, 
1997; Flores et al., 1999; 2000, 2003; Bollman et al., 1998; Beaufort et al., 1999; Beaufort and Buchet, 2003; Hagino and Okada, 2004) versus the lower photic zone dwellers (F. profunda; e.g., Okada and Honjo, 1973; Okada and McIntyre, 1977; Molfino and McIntyre, 1990a, b). High N ratio values (close to 1 ) indicate a high production of upper photic species against the production of lower photic species, as result of a shallow nutri-thermocline. Low $\mathrm{N}$ ratio values (close to 0 ) reveal a proliferation of $F$. profunda, in agreement with a deep stratification and a deep nutrithermocline (Okada and Honjo, 1973; Okada, 1980; Molfino and Mclntyre, 1990a, b; Wells and Okada, 1997; Flores et al., 2000). The accumulation rate of coccoliths (NAR, liths ${ }^{*} \mathrm{Cm}^{-2^{*}} \mathrm{Ka}^{-1}$ ) has been widely used by several authors as a reference for high palaeoproductivity of coccolithophores and particle flux out of the mixed layer (Steinmetz, 1994 and references therein; Su, 1996; Baumann et al., 2004). This parameter was estimated following the standard method of Flores and Sierro (1997), and it involves knowledge of the absolute abundances of coccoliths, the sedimentation rate and the dry sediment bulk density.

\subsubsection{Taxonomic information}

The systematic classification of coccoliths is based on their morphology. The morphological differences used for the genus Gephyrocapsa were adopted from Flores et al. (2000) and are summarised in Appendix of chapter 8. Small placoliths (such as Gephyrocapsa aperta and G. ericsonii, small Reticulofenestra and Emiliania huxleyi) with an open or closed central area and smaller than $3 \mu \mathrm{m}$ were classified together in the group of small Noelaerhabdaceae. Gephyrocapsa spp. contain Gephyrocapsa species larger than $3 \mu \mathrm{m}$ such as G. oceanica, $G$. muellerae, and $G$. caribbeanica. Different sizes of $G$. oceanica were also considered for carbonate calculations (medium Gephyrocapsa 3-4 $\mu \mathrm{m}$ and large Gephyrocapsa 4-5 $\mu \mathrm{m}$ ), but later on these sizes were lumped together as G. oceanica for palaeoecological purposes. Additionally, Oolithotus spp., Pontosphaera spp., Rhabdosphaera clavigera, Syracosphaera spp., Umbellosphaera spp. and Umbilicosphaera spp. were included as the group of warm taxa (Hiramatsu and De Deckker, 1997; Böeckel and Baumann, 2004). The genus Umbilicosphaera includes $U$. hulburtiana, $U$. foliosa and $U$. sibogae. The marked dominance of Umbilicosphaera sibogae in the group of the warm taxa suggests that its palaeoecological behaviour in Guyana Basin should be analyzed. Calcidiscus leptoporus and Helicosphaera spp. species are characterized by their lower abundances and poor consistence along the record studied. Helicosphaera spp. mainly include Helicosphaera carteri and in a very lower proportion $H$. pavimentum $(<0.5 \%)$, but we only used the record of $H$. carteri for palaeoecological purposes. 


\subsection{Presenvation}

The dissolution of the calcareous nannofossil assemblage was estimated through the CEX' index (Fig. 7.3). That index provides a qualitative record of the coccolith lysocline position (Dittert et al., 1999; Böeckel and Baumann 2004; Böeckel et al., 2006). Higher CEX' (values close to 1) point to a better preservation of the coccoliths, while lower CEX' (values close to 0 ) point to coccolith carbonate dissolution (Fig. 7.3).

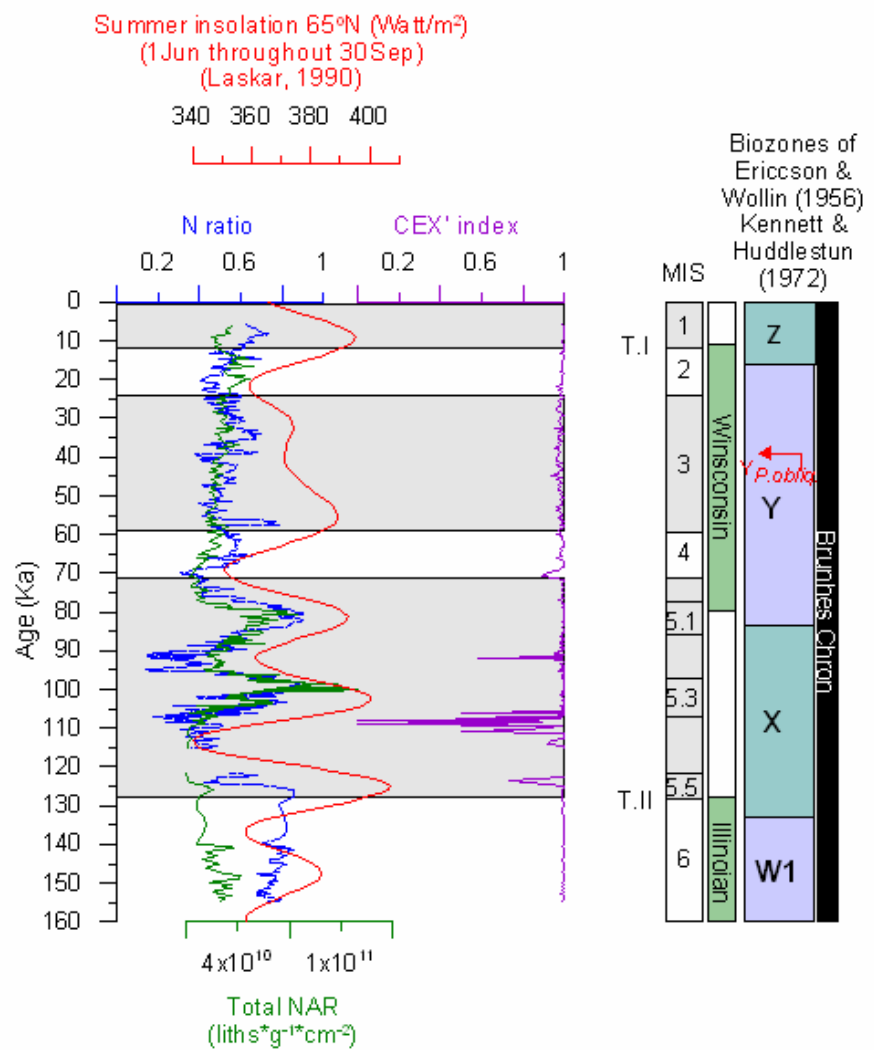

Fig. 7.3. Review of the bioproductivity proxies used for Core MD03-2616: $\mathrm{N}$ ratio vs. total nannofossil accumulation rates (NAR, liths* ${ }^{*} \mathrm{gr}-1^{*} \mathrm{Cm}-2$ ). Grey bars indicate interglacial marine isotope stages 1 throughout 5. MIS 5.1, 5.2, 5.3, 5.4, 5.5 correspond to the substages of MIS 5. Wisconsin and Illinoian episodes correspond to the Ice Ages between $12 \mathrm{Ka}$ and $80 \mathrm{Ka}$ in the former, and $128 \mathrm{Ka}$ and $310 \mathrm{Ka}$ in the later. T.I=Termination I, T.II= Termination II.

This ratio was originally based on the relationship of delicate coccoliths of Emiliania huxleyi versus the stronger calcified coccoliths of Calcidiscus leptoporus (CEX index, Dittert et al., 1999). Later, CEX index was modified by Böeckel and Baumann (2004) including other fragile species such as, Gephyrocapsa ericsoni and was called CEX'. We employed CEX' index adding the more fragile species such as: G. ericsoni, E. huxleyi and small Reticulofenestra $(<3 \mu \mathrm{m})$, since calcareous nannofossil analysis under the light microscope does not allow identification of the specimens smaller than $3 \mu \mathrm{m}$ at the species level. It is difficult to separate $G$. ericsoni that has lost its bridge from small Reticulofenestra. Although the highly calcified Gephyrocapsa aperta 
species was not included in the CEX' index, we still maintained the original concept of relating delicate coccoliths versus $C$. leptoporus.

The overall preservation of coccoliths was good along the last climatic cycle, except during the end of MIS 5.5 and MIS 5.4, which show stronger dissolution and barren intervals; and during middle of MIS 5.2, which shows an intermediate level between moderate to poor preservation. Decreases in the CEX' index and marked increases in the relative abundances of the most resistant species in the assemblage (G. oceanica and $F$. profunda) confirmed severe dissolution during later MIS 5.5 and during MIS 5.4 (Fig. 7.3)

\subsection{Results}

\subsubsection{Variations in the assemblage}

The small Noelaerhabdaceae, F. profunda and G. oceanica proved to be the dominant species in the calcareous nannoplankton assemblage (Fig. 7.4; Appendices A, B, C and Appendix of chapter 8). Gephyrocapsa muellerae and Umbilicosphaera sibogae species were identified as important secondary components. Helicosphaera spp. and C. leptoporus were considered subordinate species because they were present in lower proportion (lesser than 2\%) along the record studied

The most prominent feature of the nannoplankton record was the inverse relationship between small Noelaerhabdaceae and F. profunda. Maximum proportions and NAR values of the small Noelaerhabdaceae were found during interglacial substages $\left(80 \%\right.$ and $1 \times 10^{11}$ liths $^{*} \mathrm{~g}^{-{ }^{*}} \mathrm{~cm}^{-2}$ respectively). Higher proportions of $F$. profunda occur during glacial substages of MIS $5(80 \%)$ but its specific NAR values were observed between MIS 5.3 to $5.1\left(3 \times 10^{10}\right.$ liths $\left.^{*} \mathrm{~g}^{-1 *} \mathrm{~cm}^{-2}\right)$. Conversely, during MIS 4 and extending to the early Holocene, those taxa exhibit less amplitude (lower gradients) but more frequent variability in their relative abundances and their specific lower NAR values. F. profunda dominates the assemblage during MIS 4 to the middle of MIS 2, with mean values close to $45 \%$. F. profunda-specific NAR values show a slight increasing trend towards MIS 2 which declines during the early Holocene. Small Noelaerhabdaceae reach higher proportions from the middle of MIS 2 to the early Holocene (up to $60 \%$ ). 
G. oceanica, U. sibogae, C. leptoporus and H. carteri also show common stronger NAR gradients during MIS 5. Their specific NAR values follow those of small Noelaerhabdaceae during that stage. The relative abundances of $G$. oceanica exceed $30 \%$ when the assemblage underwent stronger dissolution (the end of MIS 5.5 and during MIS 5.4; Fig. 7.4). Other important proportion peaks of $G$. oceanica were recorded during MIS 3.1. U. sibogae also shows higher relative abundances during MIS 4 and 3 , and C. leptoporus during MIS 3.

The overall trend of all species-specific NAR values show more steady values during MIS 4 to MIS 2 and evidence a declining trend during early Holocene (except those of small Noelaerhabdaceae).

\subsubsection{Variations in the $\mathbf{N}$ ratio and total NAR profiles}

The variation in total NAR parallels that of the $\mathrm{N}$ ratio, showing higher amplitude changes between the interglacial and glacial substages of MIS 5 , with values between 0.9 to 0.2 for the $\mathrm{N}$ ratio and, $1.2 \times 10^{11}$ and $1 \times 10^{10}$ liths $^{*} \mathrm{Cm}^{-2 *} \mathrm{Ka}^{-1}$ for the total NAR. Frequent and mild fluctuations in the $\mathrm{N}$ ratio and total NAR occur throughout MIS 4-2 and the early Holocene. Those profiles are comparable to those observed at the end of MIS 5.2 (Fig. 7.4). These parameters are strongly influenced by the dominant species in the assemblage and in consequence display low gradients during glacial MIS 4-2 (Figs. 7.3 and 7.4).

The variation in palaeoproductivity reveals two different hydrographic scenarios during the last climatic cycle: the first starts at the end of MIS 6 to MIS 5, and the second spans MIS 4 to the early Holocene.

\subsection{Disc ussion}

\subsubsection{Productivity of calcareous nannoplankton}

Our data indicate lower primary production and a deep stratified photic layer during the glacial substages of the last climatic cycle (Figs. 7.1, 7.3 and 7.4). This scenario is linked to a southward displacement of the ITCZ and northeast Trade winds blowing perpendicularly to the Guyana coast, favouring the piling up of warm surface waters dragged by the NEC along this coast. In consequence, the nutri-thermocline is depressed, preventing the coastal upwelling and thus, the 
Summer insolation 65N (Watt/m²)

(1.Jun throughout 30Sep)

(Laskar, 1990)

340360380400

$\llcorner, \perp, \perp, \perp$ $\%$

small Noelaerhabdaceae

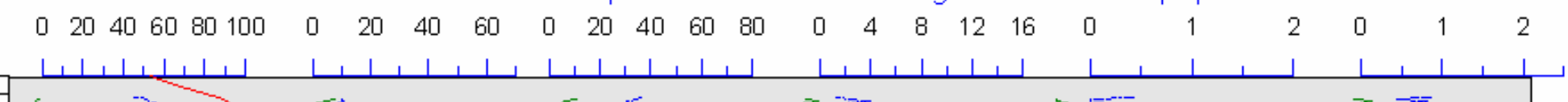

$\%$ $\%$ $\%$

F. profunda

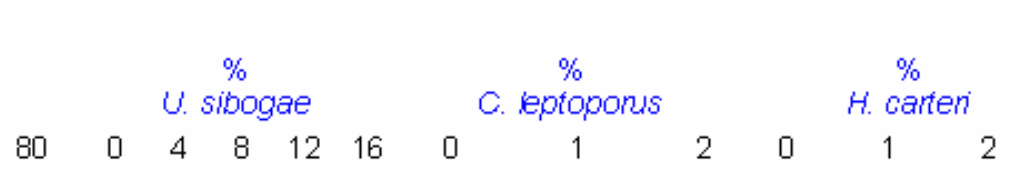
Biozones of
Ericcson \&
Wollin (1956)
Kennett \&

MIS

(1972)
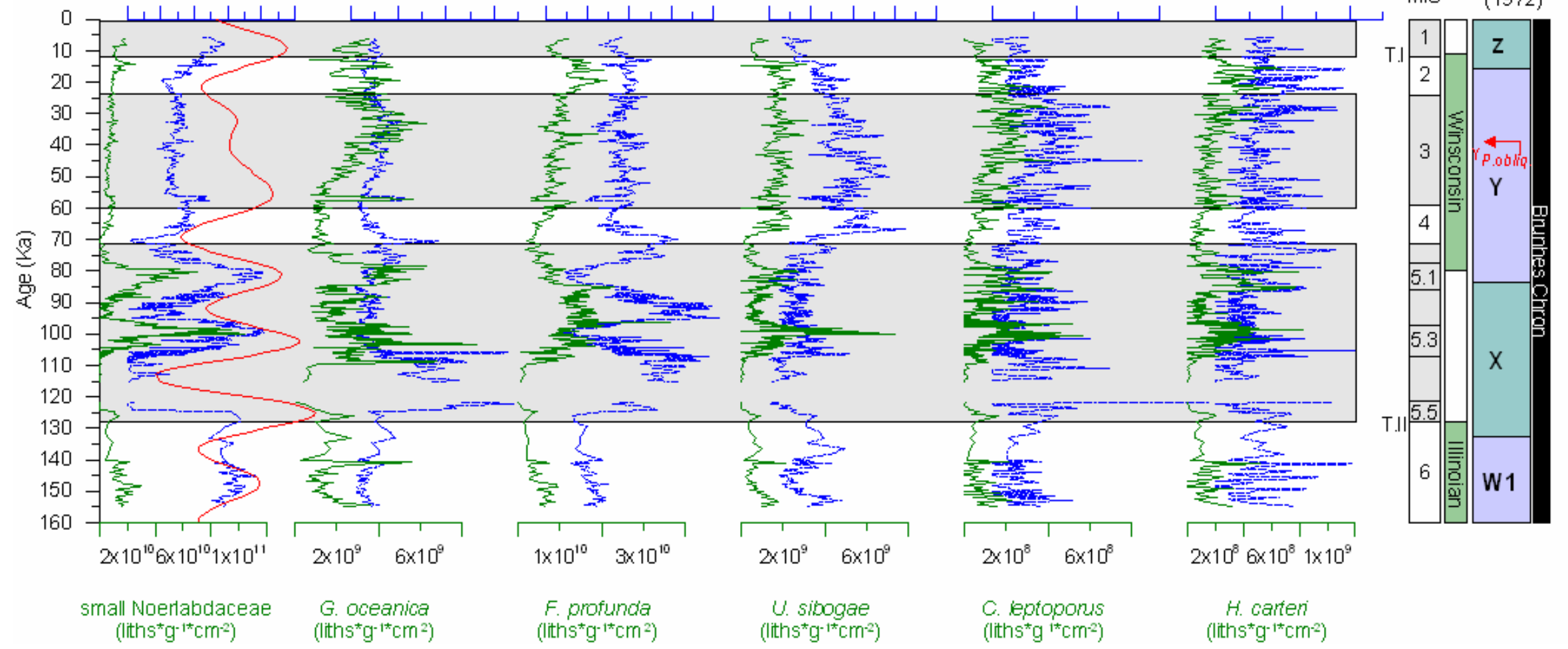

Fig. 7.4. Review of the bioproductivity proxies used for Core MD03-2616: $N$ ratio vs. total nannofossil accumulation rates $\left(\mathrm{NAR}^{2}\right.$, liths $\left.{ }^{\star} \mathrm{gr}^{1 \star} \mathrm{Cm}^{2}\right)$. Grey bars indicate interglacial marine isotope stages 1 throughout 5. MIS 5.1, 5.2, 5.3, 5.4, 5.5 correspond to the substages of MIS 5. Wisconsin and Illinoian episodes correspond to the Ice Ages between 12 Ka and 80 Ka in the former, and $128 \mathrm{Ka}$ and $310 \mathrm{Ka}$ in the later. T.I=Termination I, T.II= Termination II. 
inflow of nutrient rich and cooler waters to the euphotic zone. These atmospheric and hydrological conditions are similar to those prevailing today during boreal winter when the ITCZ is placed between $0^{\circ}$ to $5^{\circ} S$ (Müller-Karger, 1989; well south of the Guyana basin) and the northeast Trade winds blow onshore. Several authors have indicated severe Northeast Trade winds and a southward latitudinal migration of the atmospheric and hydrographic circulation in the WTA during glacial substages of MIS 3 and during MIS 2 (Balsam et al., 1995; Harris and Mix, 1999; Broccoli, 2000; Peterson et al., 2000; Vink et al., 2001, 2002). Even a southward displacement of $8^{\circ}$ for the ITCZ during MIS 2 was reported (Kolla et al., 1979).

In contrast, higher primary production and eutrophic conditions were recorded during the interglacial substages of the last climatic cycle, which are primarily the result of coastal upwelling, induced by a northward migration of the ITCZ. The ITCZ shift would follow parallel to the northeastern Southamerica coast, channelling the southeast Trade winds along the coast (Figs. 7.1, 7.3 and 7.4). That along-shore wind vector favored the eastward water transport through a well-developed NECC (Fig. 7.1). During those episodes, the piling up of warmer waters in the Guyana Basin was reduced because the studied area was located outside the influence of the northeast Trade winds. This scenario is similar to that prevailing today in the Guyana basin during boreal summer. The northward displacement of the ITCZ $\left(6^{\circ} \mathrm{N}-10^{\circ} \mathrm{N}\right)$ and the dominance of the southeast Trade winds induce coastal upwelling in the Guyana basin during summer, favoring the development of the NBC retroflection (Ryther et al., 1967; Hulburt and Corwin, 1969; MüllerKarger et al., 1989, 1995) and the formation of the NECC between $5^{\circ} \mathrm{N}-8^{\circ} \mathrm{N}$, transporting warm water offshore (Busalacchi and Picaut, 1983). Gibbs (1980), Pujos and Froidefond (1995, and references therein) have reported seasonal scenarios where the southeast Trade winds prevail over northeast Trades and blow alongshore the Guyana coast during boreal summer, promoting coastal upwelling in the basin. Color reflectance and chemistry data from the Cariaco Basin (Peterson et al., 2000) and micropalaeontological data from the Tobago Basin (Vink et al., 2001) are consistent with a northward shift of the ITCZ during interglacial substages of MIS 3. Titanium and iron content in sediments from the Cariaco Basin also evidence a northward position of the ITCZ during the early Holocene (Haug et al., 2001).

Following the same line of evidence, the micropalaeontological signal also indicates that the maximum palaeoproductivity and hence, a shallower nutri-thermocline occurred during the interglacial substages of MIS 6-5 compared with those of MIS 4-2 and with the early Holocene, 
pointing to a relative stronger coastal upwelling in the Guyana Basin during interglacial substages of MIS 6-5 that was induced by a more northern position of the ITCZ during the same episodes.

\subsubsection{The nutri-themocline dynamic and the Northem Hemisphere connection}

The section above cited that maximum palaeoproductivity is in agreement with the timing of prevailing southeast Trade winds which cause the northward movement of the ITCZ. Productivity phases are coincident with the northern insolation curve (mean insolation, June 1 to September $30,65^{\circ} \mathrm{N}$ ) in timing and amplitude, since a shallow nutri-thermocline follows the maximum in insolation at $65^{\circ} \mathrm{N}$. This correlation suggests a close connection between the shifts of the nutrithermocline, Trade wind dynamics, and the latitudinal migration of the ITCZ. Northward shift of the ITCZ and the rain belt embedded in it are influenced by the meridional wind component which in turn is the response of the sea surface temperature and salinity gradients between high and low latitudes in the Northern Hemisphere (Hastenrath and Druyan, 1993; Hastenrath and Greischar, 1993). Those gradients are induced by insolation change controlling the ice sheet volume in the Arctic (Khodri et al., 2003), which in turn control Trade wind intensity (Chiang et al., 2003). In a similar manner, changes in the temperature/pressure gradients would also explain larger fluctuations in palaeoproductivity during warmer MIS 5 compared to colder MIS 4-2.

Molfino and Mclntyre (1990a, b) and Wolff et al. (1999) found that variations in the position of the nutri-thermocline are controlled by the Trade winds in the eastern tropical Atlantic (but in contrast with the present findings in the WTA, they reported a shallow nutri-thermocline during glacial periods). Kinkel et al. (2000) recorded a shallow nutricline and high coccolithophore productivity along the interglacial times and a deeper nutri-thermocline along the glacial stages and substages in the WTA during the last $150 \mathrm{Ka}$. Geochemical and sedimentological evidence from the Ceara Rise also support a shallow nutri-thermocline during interglacial substages during the last climatic cycle (Rühlemann et al., 2001) which is in good agreement with our data.

Variations in the position of the thermocline have been reported by Hüls and Zahn (2000) and Vink et al. (2001) in a nearby core taken from the Tobago Basin. They found the shallowest thermocline for MIS 3 ( $\sim 50 \mathrm{~m}$ depth) in comparison with the glacial MIS 2 ( 80 m depth) and the Holocene (150 m depth). Pailler et al. (1999) reported that the main thermocline is currently located at $150 \mathrm{~m}$ deep in a section of the WTA, where our study was performed. 


\subsubsection{River nunoff}

The main freshwater source that affects the WTA is the Amazon River, which is mostly responsible for the low sea surface salinity in the WTA (Masson and Delecluse, 2000), since the waters of the Orinoco flow downstream towards the Caribbean Sea (Ffield, 2005). Several authors (Müller-Karger et al., 1989 and references therein; Peterson et al., 2000) have indicated that high abundances of phytoplankton are associated with higher seasonal river runoff and lower salinities in the northern Caribbean Sea $\left(14^{\circ} \mathrm{N}\right.$ and $\left.18^{\circ} \mathrm{N}\right)$ than in the typical waters of the WTA.

The association in temperature and pressure gradients between low and high latitudes, and the ITCZ position cited above, accounts for the relationship between cold/warm periods in high latitudes in the North Atlantic and drier/wetter conditions over northern South America (Haug et al., 2001). The northward position of the ITCZ during the interglacial substages over the last climatic cycle favors the influx of river nutrients to the ocean and the configuration of an oceanic system that supports a superficial nutri-thermocline and consequently increased biological productivity in the Cariaco Basin (Peterson et al., 2000), Trinidad Basin (Vink et al., 2001), and Caribbean Sea (Martínez et al., 2007). Similarly, Showers and Bevis (1988) documented an increase in the Amazon River runoff during Termination I until 5-6 Ka B.P. ago. Other authors have reported the southward geographic situation of the ITCZ over the last glacial and consequently drier conditions over northern South America (Kolla et al., 1979; Balsam et al., 1995; Peterson et al., 2000), and decreases in the influence of the river flow system and a deeper nutri-thermocline that reduced bioproductivity (Peterson et al., 2000; Vink et al., 2000; 2001; 2002).

According with these suggestions and the chlorophyll concentration data (primary productivity distribution) presented by SeaWIFS Project imagery (Fig. 7.1), the influence of an enhanced nutrient supply by rivers in the production of calcareous nannoplankton cannot be completely ruled out. However, there is no clear indicator within the association of calcareous nannoplankton able to account for a greater discharge from the Amazon during the interglacials, e.g., reworked individuals or even freshwater-related species (phytoliths or freshwater diatoms), and there is no evidence in the association of low sea surface salinity. $U$. sibogae has been directly related to surface water salinity in the North Pacific (Roth and Coulbourn, 1982), and has been found in warm oligotrophic waters (Okada and Mclntyre, 1979; Roth, 1994; Young, 1994; Böeckel and Baumann, 2004; Ziveri et al., 2004), but also in medium-to-high fertility waters (Roth and Berger, 
1975 ; Roth and Coulbourn, 1982) with temperatures ranging from $18^{\circ} \mathrm{C}$ to $25^{\circ} \mathrm{C}$ (Mclntyre and Bé, 1967; Okada and McIntyre, 1979). G. oceanica has also been observed in relatively warm, highly saline and fertile waters (Winter, 1982, 1985; Mitchell-Innes and Winter, 1987). H. carteri has been recorded during low salinity and turbid water settings (Colmenero-Hidalgo et al., 2004) and also in eutrophic (Ziveri et al., 1995; Ziveri et al., 2000; Andruleit and Rogalla, 2002; Findlay and Giraudeau, 2002; Ziveri et al., 2004; Baumann et al., 2005) and oligotrophic (Haidar and Thierstein, 1997; Giunta et al., 2003) environments. The palaeocological preference of $C$. leptoporus has been related to elevated nutrient levels (Roth and Berger, 1975; Fincham and Winter, 1989; Andruleit and Rogalla, 2002; Flores et al., 2003) and also with more oligotrophic and warmer conditions (Flores et al., 1999; Baumann et al., 2004; Ziveri et al., 2004; Marino et al., 2008). However, U. sibogae, G. oceanica, H. carteri and C. leptoporus do not show a systematic correspondence with temperature or salinity. This implies that $U$. sibogae, $G$. oceanica, $\mathrm{H}$. carteri and $\mathrm{C}$. leptoporus respond to a productivity pattern rather than to a pattern of temperature or salinity, especially significant in MIS 4-2 (Fig. 7.3).

\subsubsection{Dissolution of calcareous nannoplankton}

Coccolith dissolution episodes (Figs. 7.3 and 7.4) suggest either a shallowing of the lysocline or dissolution above the lysocline. Curry and Cullen (1997) have documented high carbonate dissolution during glacial periods in response to deep water mass changes at greater depths in the WTA (>3000 m water depth). Owing to the shallow depth position of Core MD03-2616, dissolution below the lysocline is ruled out, because aragonite and calcite lysoclines have been reported at 2200 and $4000 \mathrm{~m}$ respectively (in Vink et al., 2001). Dissolution above the lysocline may be a response to the acidification of interstitial waters due to the oxidation of organic matter within the sediments. However, this hypothesis is not supported by the available observations, and hence its confirmation requires detailed benthonic isotope $\delta^{13} \mathrm{C}$ data, sedimentological studies, and further oceanographic reconstructions in the Guyana Basin.

\subsection{Conclusions}

The evolution of the calcareous nannoplankton assemblage is mainly a response to a productivity pattern in the Guyana Basin. The data on the NAR record resemble those of the N ratio when dissolution is lacking, suggesting that NAR is also indicative of variations in the palaeoproductivity pattern. 
A high palaeoproductivity and a shallow nutri-thermocline during interglacial substages over the past $155 \mathrm{Ka}$ result from the dominant southeast Trade winds that displaced the ITCZ to a more northern position and blew parallel to the coast, causing upwelling of cool waters from depth. Decreases in palaeoproductivity during glacial substages would have resulted from dominant northeast Trade winds that moved the ITCZ southward and blew perpendicular to the coast causing piling up of the NEC waters and hence, the deepening of the nutri-thermocline in the Guyana Basin.

The micropalaeontological data reveals that the highest palaeoproductivity and hence, the shallowest nutri-thermocline occurred during the interglacial substages of MIS 6-5 compared with those interglacial episodes of MIS 4-2 and with the early Holocene. This record indicates more eutrophic conditions during the interglacial events of MIS 6-5 which were favored by a more northern position of the ITCZ during the same episodes.

The production of calcareous nannoplankton and the shifting of the nutri-thermocline covary with summer insolation at high northern latitudes. Low palaeoproductivity was recorded during periods of low northern summer insolation (glacial substages of the last climatic cycle) indicating that during times of higher ice volume, the northeast Trade winds were dominant, moving the ITCZ southward and causing the deepening of the nutri-thermocline in the Guyana Basin. High palaeoproductivity was recorded during times of high Northern Hemisphere insolation (interglacial substages over the last climatic cycle) suggesting that southeast Trade winds prevailed over the northeast Trades, moved the ITCZ northward, permitted the development of the coastal upwelling and hence, the shallowing of the nutri-thermocline.

Higher palaeoproductivity during the northern position of the ITCZ coincide with the timing of episodes of larger runoff. Calcareous nannoplankton data did not record variability related to the larger river discharges. However, the influence of continental-derived nutrients and a freshening of surface water masses in the ocean through Amazon River runoff cannot be discounted.

\section{Aknowledgements}

The research discussed here was supported by Spanish projects CGL2005-00642/BTE, CGL2006-10593 from the Ministerio de Educación y Ciencia, A008C05 from the Consejería de Educación de la Junta de Castilla y León and by the Spanish Grant BES-2003-0010 from the 
Ministerio de Educación y Ciencia to G.-E. López-Otálvaro. We also gratefully thank M. A.

Bassetti, Gabriel Filippelli and one anonymous reviewer for their useful comments.

\section{References}

Andruleit, H.A., Rogalla, U. 2002. Coccolithophores in surface sediments of the Arabian Sea in relation to environmental gradients in Surface waters. Marine Geology 186, 505-526.

Balsam, W.L., Otto-Bliesner, B.L., Deaton, B.C. 1995. Modern and last glacial maximum eolian sedimentation patterns in the Atlantic Ocean interpreted from iron oxide content, Paleoceanography, 10, 493-507.

Bassinot, F. C., Beaufort, L., Vincent, E., Labeyrie, L. 1997. Changes in the dynamics of Western Equatorial Atlantic surface currents and biogenic productivity at the "Mid-Pleistocene Revolution" ( 930 Ka): Leg 154. In: Shackleton, N. J., Curry, W. B., Richter, C., Bralower, T. J. (Eds.). Proc. ODP, Sci. Results, 154: College Station, TX (Ocean Drilling Program), pp. 269-284.

Baumann, K.-H., Freitag, T. 2004. Pleistocene fluctuations in the northern Benguela Current systems as revealed by coccolith assemblages. Marine Micropaleontology 52, 195-215.

Baumann, K.-H., Böeckel, B., Frenz, M. 2004. Coccolith contribution to South Atlantic carbonate sedimentation. In: Thierstein, H., Young, J. (Eds.), Coccolithophores - From Molecular Processes to Global Impact. Springer-Verlag, 367-402.

Baumann, K.-H., Andruleit, H., Bockel, B., Geisen, G., Kinkel, H. 2005. The significant of extant coccolithophores as indicators of ocean water masses, surface water temperature, and paleoproductivity: a review. Paläontologische Zeitschrift 79, 93-112.

Beaufort, L., Buchet, N. 2003. Variability of specific coccolith $\mathrm{CaCO}_{3}$ weight, and primary production in the western Pacific warm pool during the last glacial cycle. Geophysical Research Abstracts (European Geosciences Union) 5, 13225.

Beaufort, L, Lancelot, Y., Camberlin, P., Cayre, O., Vincent, E., Bassinot, F., Labeyrie, L. 1997. Insolation Cycles as a Major Control of Equatorial Indian Ocean Primary Production. Science 278, 14511454.

Beaufort, L., Bassinot, F.C., Vincent, E. 1999. Primary production response to orbitally induced variations of the Southern Oscillation in the Equatorial Indian Ocean. In: Abrantes, F., Mix, A.C. (Eds.), Reconstructing Ocean History: a Window into the Future. Kluwer Academic/Plenum, New York, pp. 245-272.

Beaufort, L. de Garidel-Thoron, T., Mix, A.C., Pisias, N.G. 2001. ENSO-like Forcing on Oceanic Primary Production During the Late Pleistocene. Science 293, 2440-2444.

Beaufort, L., de Garidel-Thoron, T., Linsley B., Oppo D., Buchet N. 2003. Biomass burning and oceanic primary production estimates in the Sulu Sea area over the last $380 \mathrm{Ky}$ and the East Asian monsoon dynamics. Marine Geology 201, 53-65.

Böeckel, B., Baumann, K.-H. 2004. Distribution of coccoliths in surface sediments of the south-eastern South Atlantic Ocean: ecology, preservation and carbonate contribution. Marine Micropaleontology 51, 301-320.

Böeckel, B., Baumann, K.-H., Henrich, R., Kinkel, H. 2006. Coccolith distribution patterns in South Atlantic and Southern Ocean surface sediments in relation to environmental gradients. Deep-Sea Research 53, 1073-1099.

Bollmann, L., Baumann, K.-H., Thierstein, H.R. 1998. Global dominance of Gephyrocapsa coccoliths in the Late Pleistocene: Selective dissolution, evolution or global environment change. Paleoceanography $13,517-529$.

Brand, L.E. 1994. Physiological ecology of marine coccolithophores. In: Winter, A., Siesser, W.G. (Eds.), Coccolithophores. Cambridge Univ. Press, Cambridge, U.K., 39-49.

Broccoli, A.J. 2000. Tropical cooling at the Last Glacial Maximum: an atmosphere-mixed layer ocean model simulation. Journal of Climate 13, 951-976.

Busalacchi, A., Picaut, J. 1983. Seasonal variability from a model of the tropical Atlantic Ocean. Journal of Physical Oceanography 13, 1564-1588. 
Chiang, J.C., Biasutti, M., Battisti, D.S. 2003. Sensitivity of the Atlantic Intertropical Convergence Zone to Last Glacial Maximum boundary conditions. Paleoceanography 18, doi: 10.1029/2003PA000916.

Colmenero-Hidalgo, E., Flores, J.A., Sierro, J., Barcena, M.A., Lowemark, L., Schonfeld, J., Grimalt, J.O. 2004. Ocean surface water response to shortterm climate changes revealed by coccolithophores from the Gulf of Cadiz (NE Atlantic) and Alboran Sea (W Mediterranean). Palaeogeography, Palaeoclimatology, Palaeoecology 205, 317-336.

Curry, W.B., Cullen, J.L. 1997. Carbonate production and dissolution in the western equatorial Atlantic during the last 1 M.Y.: Leg 154. In: Shackleton, N.J., Curry, W.B., Richter, C. Bralower, T.J. (Eds.). Proc. ODP, Sci. Results, 154: College Station, TX (Ocean Drilling Program), pp. 189-199.

da Silveira, I. C. A., de Miranda, L. B., Brown, W. S. 1994. On the origins of the North Brazil Current, Journal of Geophysical Research 99, 22501-22512.

de Menocal, P.B. 1995. Plio-Pleistocene African Climate. Science 270, 53-59.

Dittert, N., Baumann, K.-H., Bickert, R., Henrich, R., Huber, R., Kinkel, H., Meggers, H. 1999. Carbonate dissolution in the deep-sea: methods, quantification and paleoceanographic application. In: Fischer, G. and Wefer, G., Editors, 1999. Use of Proxies in Paleoceanography: Examples from the South Atlantic, Springer Verlag, Berlin, Heidelberg, 255-284.

Ericson, D.B., Wollin, G. 1956. Correlation of six cores from the equatorial Atlantic and the Caribbean. Deep-Sea Research 3, 104-125.

Ffield, A. 2005. North Brazil current rings viewed by TRMM Microwave Imager SST and the influence of the Amazon Plume. Deep-Sea Research I 52, 137-160.

Fincham, M.J., Winter, A. 1989. Paleoceanographic interpretation of coccoliths and oxygen isotopes from sediments from the surface of Southwest Indian Ocean. Marine Micropaleontology 13, 325-351.

Findlay, C.S., Giraudeau, J. 2000. Extant calcareous nannoplankton in the Australia sector of the southern ocean (Austral summer 1994 and 1995). Marine Micropaleontology 40, 417-439.

Flores, J.A., Sierro, F.J. 1997. Revised technique for calculation of calcareous nannofossil accumulation rates. Micropaleontology 43, 321-324.

Flores, J.-A., Gersonde, R., Sierro, F.-J. 1999. Pleistocene fluctuations in the Agulhas Current Retroflection based on the calcareous plankton record. Marine Micropaleontology 37, 1-22.

Flores, J.A., Bárcena, M.A., Sierro, F.J. 2000. Ocean-surface and wind dynamics in the Atlantic Ocean off Northwest Africa during the last 140,000 years. Palaeogeography, Palaeoclimatology, Palaeoecology 161, 459-478.

Flores, J.A., Marino, M., Sierro, F.J., Hodell, D.A., Charles, C.D. 2003. Calcareous plankton dissolution pattern and coccolithophore assemblages during the last 600 kyr at ODP Site 1089 (Cape Basin, South Atlantic): paleoceanographic implications. Palaeogeography, Palaeoclimatology, Palaeoecology 196, 409-426.

Gibbs, R.J. 1980. Wind-controlled coastal upwelling in the western equatorial Atlantic. Deep-Sea Research 27A, 857-866.

Gibbs, S, Shackleton, N, Young, J. 2004. Orbitally forced climate signals in mid-Pliocene nannofossil assemblages. Marine Micropaleontology 51, 9-56.

Giraudeau, J. 1992. Distribution of Recent nannofossils beneath the Benguela system: Southwest African continental margin. Marine Geology 108, 219-237.

Giunta, S., Negri, A., Morigi, C., Capotondi, L., Combourieu-Nebout, N., Emeis, K.C., Sangiorgi, F., Vigliotti, L. 2003. Coccolithophorid ecostratigraphy and multi-proxy paleoceanographic reconstruction in the Southern Adriatic Sea during the last deglacial time (Core AD91-17). Palaeogeography, Palaeoclimatology, Palaeoecology 190, 39-59.

Hagino, K., Okada, H. 2004. Floral response of coccolithophores to progressive oligotrophication in the South Equatorial Current, Pacific Ocean. In: Shiyomi, M., Kawahata, H., Koizumi, H., Tsuda, A., Awaya, Y. (Eds.), Global Environmental Change in the Ocean and on Land. TERRAPUB, 121132.

Haidar, A.T., Thierstein, H.R. 1997. Calcareous phytoplankton dynamics at Bermuda (N. Atlantic). EGU 9 Abstr. Suppl. 1. Terra Nova 9, p. 602.

Harris, S., Mix, A.C. 1999. Pleistocene precipitation balance in the Amazon Basin recorded in deep sea sediments. Quaternary Research 51, 14-26. 
Hastenrath, S., Druyan, L. 1993. Circulation anomaly mechanisms in the tropical Atlantic sector during the Northeast Brazil rainy season: results from the GISS General Circulation Model. Journal of Geophysical Research-Atmospheres 98 (D8), 14017-14923.

Hastenrath, S., Greischar, L. 1993. Circulation mechanisms related to Northeast Brazil rainfall anomalies. Journal of Geophysical Research-Atmospheres 98 (D5), 5093-5102.

Hastenrath, S., Merle, J. 1987. Annual cycle of subsurface thermal structure in the tropical Atlantic ocean. Journal of Physical Research 17, 1518-1538.

Haug, G. H., Hughen, K. A., Sigman, D. M., Peterson, L.C., Röhl, U. 2001. Southward migration of the Intertropical Convergence Zone through the Holocene. Science 293, 1304-1308.

Haug, G.H., Günther, D., Peterson, L.C, Sigman, D.M., Hughen, K.A. Aeschlimann, B. 2003. Climate and the collapse of Maya Civilization. Science 299, 1731-1735.

Hibberd, D. J. 1976. The ultrastructure and taxonomy of the Chrysophyceae and Prymnesiophyceae (Haptophyceae): a survey with some new observations on the ultrastructure of the Chrysophyceae. Botanical Journal of the Linnean Society 72, 55-80.

Hiramatsu, C., De Deckker, P. 1997. The late Quaternary calcareous nannoplankton assemblages from three cores from the Tasman Sea. Palaeogeography, Palaeoclimatology, Palaeoecology 131, $391-412$.

Hulburt, E.M., Corwin, N. 1969. Influence of the Amazon River outflow on the ecology of the western tropical Atlantic: III. The planktonic flora between the Amazon River and the Windward islands. Journal of Marine Research 27, 55-72.

Hüls, M., Zahn, R. 2000. Millennial-scale sea surface temperature variability in the western tropical North Atlantic from planktonic foraminiferal census counts. Paleoceanography, 15, 659- 678.

Kennett, J.P., Huddlestun, P. 1972. Late Pleistocene Paleoclimatology, foraminiferal biostratigraphy and tephrochronology, western Gulf of Mexico. Quaternary Research 2, 38-69.

Khodri, M., Ramstein, G., de Noblet-Ducoudré, N. 2003. Sensitivity of the northern extratropics hydrological cycle to the changing insolation forcing at 126 and $115 \mathrm{ky}$ BP. Climate Dynamics 21, 273-287. doi: 10.1007/s00382-003-0333-5.

Kinkel, H., Baumann, K.-H., Čepek, M. 2000. Coccolithophores in the equatorial Atlantic Ocean: response to seasonal and Late Quaternary surface water variability. Marine Micropaleontology 39, 87-112.

Kolla, V., Biscaye, P. E., Hanley, A. F. 1979. Distribution of quartz in late Quaternary Atlantic sediments in relation to climate. Quaternary Research 11, 261- 277.

Lazar, A., Inui, T., Malanotte-Rizzoli, P., Busalacchi, A.J., Wang, L., Murtugudde, R. 2002. Seasonality of the ventilation of the tropical Atlantic thermocline in an ocean general circulation model. Journal of Geophysical Research 107, (C8), doi:10.1029/2000JC000667.

Lisiecki, L.E., Raymo, M.E. 2005. A Pliocene-Pleistocene stack of 57 globally distributed benthic $\delta^{18} \mathrm{O}$ records. Paleoceanography 20, doi:10.1029/2004PA001071.

Liu, Z., Herbert, T. 2004. High-latitude on the Eastern Equatorial Pacific climate in the early Pleistocene epoch. Nature 427, 720-723.

López-Otálvaro, G.-E., Flores, J.-A., Sierro, F.-J., Cacho, I. 2008. Variations in coccolithophorid production in the Eastern Equatorial Pacific at ODP Site 1240 over the last seven glacial-interglacial cycles. Marine Micropaleontology. doi: 10.1016/j.marmicro.2007.11.009.

Lynch-Stieglitz, J., Curry, W.B., Slowey, N. 1999. Weaker Gulf Stream in the Florida Straits during the Last Glacial Maximum. Nature 402, 644-648.

Marino, M., Maiorano, P., Lirer, F. 2008. Changes in calcareous nannofossil assemblages during the MidPleistocene Revolution. Marine Micropaleontology doi:10.1016/j.marmicro.2007.11.010.

Marsh, M.E. 2003. Regulation of $\mathrm{CaCO}_{3}$ formation in coccolithophores. Comparative Biochemistry and Physiology 136, 743-754.

Martínez, J. I., Mora, G., Barrows, T.T. 2007. Paleoceanographic conditions in the western Caribbean Sea for the last $560 \mathrm{Ka}$ as inferred from planktonic foraminifera. Marine Micropaleontology 64, 177188.

Masson, S. Delecluse, P. 2000. Influence of the Amazon river runoff on the tropical Atlantic. Physics and Chemistry of the Earth 26, 137-142.

Maslin, M., Mikkelsen, N. 1997. Amazon Fan mass transport deposits and underlying interglacial deposits: age estimates and fan dynamics: Leg 155. In: Flood, R.D., Piper, D.W.J., Klaus, A., Peterson, 
L.C. (Eds.). Proc. ODP, Sci. Results, 155: College Station, TX (Ocean Drilling Program), 353365.

McIntyre, A., Bé, A.W.H. 1967. Modern Coccolithophoridae of the Atlantic Ocean-1. Placoliths and cyrtoliths. Deep-Sea Research 14, 561-597.

Milliman, J.D. 1993. Production and accumulation of calcium carbonate in the ocean: budget of an unsteady state. Global Biogeochemical Cycles 7, 927-957.

Mitchell-Innes, B.A., Winter, A. 1987. Coccolithophores: a major phytoplankton component in mature upwelled waters off the Cape Peninsula, South Africa in March, 1983. Marine Biology 95, 25-30.

Molfino, B., Mclntyre, A. 1990a. Precessional forcing of nutricline dynamics in the equatorial Atlantic. Science 249, 766-769.

Molfino, B., Mclntyre, A. 1990b. Nutricline variation in the equatorial Atlantic coinciding with the Younger Dryas. Paleoceanography 5, 997-1008.

Müller-Karger, F.E., McClain, C.R., Richardson, P.L. 1988. The dispersal's of the amazon water. Nature 333, 56-59.

Müller-Karger, F.E, McClain, C.R., Fisher, T.R., Esaias, W.E., Varela, R. 1989. Pigment distribution in the Caribbean Sea. Progress in Oceanography 23, 23-64.

Müller-Karger, F.E., Aparicio-Castro, R. 1994. Mesoscale processes affecting phytoplancton abundance in the southern Caribbean Sea. Continental Shelf Research 14, 199-221.

Müller-Karger, F.E., Richardson, P.L., McGillicuddy, D. 1995. On the offshore dispersal of the Amazon's Plume in the North Atlantic: Comments on the paper by A. Longhurst, "Seasonal cooling and blooming in tropical oceans". Deep-Sea Research I 42, 2127-2137.

Okada, H. 1980. Calcareous nannofossils from Deep Sea Drilling Project Sites 442 through 446. Philippines Sea. Init. Rep. DSDP 58, 549-565.

Okada, H., Honjo, S. 1973. The distribution of oceanic coccolithophorids in the Pacific. Deep-Sea Research 20, 355-374.

Okada, H., Mclntyre, A. 1977. Modern coccolithophores of the Pacific and North Atlantic Oceans. Micropaleontology 23, 1-55.

Okada, H., McIntyre, A. 1979. Seasonal distribution of modern Coccolithophores in the Western North Atlantic ocean. Marine Biology 54, 319-328.

Okada, H., Wells, P. 1997. Late Quaternary nannofossil indicators of climate change in two deep-sea cores associated with the Leeuwin Current off Western Australia. Palaeogeography, Palaeoclimatology, Palaeoecology 131, 413-432.

Pailler, K., Bourlès, B., Gouriou, Y. 1999. The Barrier layer in the Western tropical Atlantic Ocean. Geophysical Research Letters 26, 2069-2072.

Peterson, L.C., Haug, G.H., Hughen, K.A., Röhl, U. 2000. Rapid changes in the hydrologic cycle of the Tropical Atlantic during the Last Glacial. Science 290, 1947-1951.

Prell, W.L., Damuth, J.E. 1978. The climate-related diachronous disappearance of Pulleniatina obliquiloculata in Late Quaternary sediments of the Atlantic and Caribbean. Marine Micropaleontol. 3, 267-277.

Pujos, M., Froidefond, J.-M. 1995. Water masses and suspended matter circulation on the French Guiana continental shelf. Continental Shelf Research 15, 1157-1171.

Richardson, P.L., Hufford, G., Limeburner, R., Brown, W. 1994. North Brazil Current retroflection eddies. Journal of Geophysical Research 99, 5081-5093.

Roth, P.H. 1994. Distribution of coccoliths in ocean sediments. In: Winter, A., Siesser, W.G. (Eds), Coccolithophores. Cambridge University Press, Cambridge, 199-218.

Roth, P.H., Berger, W.H. 1975. Distribution and dissolution of coccoliths in the South and central Pacific. Special Publications vol. 13. Cushman Foundation for Foraminiferal Research, 87-113.

Roth, P.H., Colbourn, W.T. 1982. Floral and solution patterns of coccoliths in surface sediments of the North Pacific. Marine Micropaleontology 7, 1-52.

Rühlemann, C., Dickmann, B., Mulitza, S., Frank, M. 2001. Late Quaternary changes of western equatorial Atlantic surface circulation and Amazon lowland climate recorded in Ceara Rise deep-sea sedimentos. Paleoceanography 16, 293-305.

Ryther, J.H., Menzel, D.W., Corwin, N. 1967. Influence of the Amazon River outflow on the ecology of the western tropical Atlantic: I. Hydrography and nutrient chemistry. Journal of Marine Research 25, 69-83. 
Schmitz, W.J. Jr. 1995. On the interbasin-scale thermohaline circulation. Reviews of Geophysics 33, 151 173.

Shipboard Scientific Party. 2003. MD132-PICASSO. IMAGES XI. Cruise Report. 76p.

Showers, W.J., Bevis, M. 1988. Amazon cone isotopic stratigraphy: evidence for the source of the tropical freshwater spike. Palaeogeography, Palaeoclimatology, Palaeoecology 64, 189-199.

Siesser, W., Winter, A. 1994. Composition and morphology of coccolithophore skeletons. In: A. Winter and W.G., Siesser (Eds.), Coccolithophores. Cambridge Univ. Press, Cambridge, U.K., pp. 51-62.

Steinmetz, J.C. 1994. Sedimentation of coccolithophores. In: Winter, A., Siesser, W.G. (Eds), Coccolithophores. Cambridge University Press, Cambridge, 179-197.

Stramma, L., Fischer, J., and Reppin, J. 1995. The North Brazil Undercurrent, Deep-Sea Research 42, 773-795.

Stramma, L., Schott, F. 1996. Western equatorial circulation and interhemispheric exchange. In: Krauss, W. (Ed.), The Warm water sphere of the North Atlantic Ocean. Gebrüder Borntraeger, Berlin, Stuttgart, 195-227.

Stramma, L., Rhein, M., Brandt, P., Dengler, M., Böning, C., Walter, M. 2005. Upper ocean circulation in the western tropical Atlantic in boreal fall 2000. Deep-Sea Research I 52, 221-240.

$\mathrm{Su}, \mathrm{X} .1996$. Development of late tertiary and quaternary coccolith assemblages in the Northeast Atlantic. GEOMAR-Report 48, 119.

Thierstein, H.R., Geitzenauer, K., Molfino, B., Shackleton, N.J. 1977. Global synchroneity of late Quaternary coccolith datum levels: validation by oxygen isotopes. Geology, 5, 400-404.

Vink, A., Zonneveld, K.A.F., Willems, H. 2000. Distributions of calcareous dinoflagellate cysts in surface sediments of the western equatorial Atlantic Ocean, and their potential use in paleoceanography. Marine Micropaleontology 38, 149-180.

Vink, A., Rühlemann, C., Zonneveld, K., Mulitza, S., Hüls, M., Willems, H. 2001. Shifts in the position of North Equatorial current and rapid productivity changes in the western Tropical Atlantic during the last glacial. Paleoceanography 16, 1-12.

Vink, A., Brune, C.H., Zonnevveld, K.A.F., Willems, H. 2002. On the response of calcareous dinoflagellates to oligotrophy and stratification of the upper water column in the equatorial Atlantic Ocean. Palaeogeography, Palaeoclimatology, Palaeoecology 178, 53-76.

Wells, P., Okada, H. 1997. Response of nannoplankton to major changes in sea-surface temperature and movements of hydrological fronts over Site DSDP 594 (south Chatham Rise, southeastern New Zeland), during the last $130 \mathrm{Kyr}$. Marine Micropaleontology 32, 341-363.

Westbroek, P., Brown, C.W., Van Bleijswijk, J., Brownlee, C., Brummer, G.J., Conte, M., Egge, J., Fernández, E., Jordan, R., Knappertsbusch, M., Stefels, J., Veldhuis, M., Van Der Waal, P., Young, J.R. 1993. A model system approach to biological climate forcing. The example of Emiliania huxleyi. Global Planetary Change 8, 27-46.

Wilson, W.D., Johns, W.E., Garzioli, S.L. 2002. Velocity structure of North Brazil current rings. Gephysical Research Letters 29, 8. doi: 1029/2001GL013869.

Winter, A. 1982. Paleoenvironmental interpretation of Quaternary coccolith assemblages from the Gulf of Aqaba (Elat), Red Sea. Revista Española de Micropaleontología 14, 291-314.

Winter, A. 1985. Distribution of living coccolithophores in the California Current system, southern California borderland. Marine Micropaleontology 9, 385-393.

Winter, A., Jordan, R.W., Roth, P. 1994. Biogeography of living coccolithophores in ocean waters. In: Winter, A., Siesser, W.G. (Eds), Coccolithophores. Cambridge University Press, Cambridge, 161-177.

Wolff, T.. Multiza, S., Rühlemann, C., Wefer, G. 1999. Response of the tropical Atlantic thermocline to late Quaternary Trade wind changes. Paleoceanography 14, 374-383.

Young, J.R. 1994. Functions of coccoliths. In: Winter, A., Siesser, W.G. (Eds), Coccolithophores. Cambridge University Press, Cambridge, 63-82.

Ziveri, P., Thunell, R.C., Rio, D. 1995. Export production of coccolithophores in an upwelling region: results from San Pedro Basin, Southern California Borderlands. Marine Micropaleontology 24, 335-358.

Ziveri, P., Thunell, R.C. 2000. Coccolithophores export production in Guaymas Basin, gulf of California: response to climatic forcing. Deep-Sea Research II 47, 2073-2100. 
Ziveri, P., Baumann, K.-H., Böeckel, B., Bollman, J., Young, J. 2004. Present day coccolithophorebiogeography in the Atlantic Ocean. In: Thierstein, H., Young, J. (Eds.). Coccolithophores - From Molecular Processes to Global Impact. Springer-Verlag, 403-428. 


\title{
Síntesis y Perspectiva Regional
}

\author{
8.1. Introducción
}

8.2. Modelo de edad y bioestratigrafía

8.2.1. Océano Pacífico oriental tropical

8.2.2. Océano Atlántico occidental tropical

8.3. Preservación

8.4. La variabilidad de la nutri-termoclina: el patrón glacial/interglacial en la cuenca de Panamá 8.5. Eventos análogos a "El Niño" a escala milenaria?

8.6. Influencia de la insolación en la posición de la nutri-termoclina en el Atlántico occidental tropical

8.7. Consideraciones finales

Apéndice: Consideraciones paleoecológicas de la asociación cocolitoforal Referencias 


\subsection{Introducción}

En este capítulo se presenta una síntesis y una perspectiva regional de las investigaciones presentadas en los capítulos 5, 6 y 7 que conforman los objetivos de esta Tesis Doctoral. La comparación interlatitudinal de la evolución de la asociación de los cocolitóforos en los sectores tropicales de los océanos Pacífico oriental (cuenca de Panamá) y Atlántico occidental (cuenca de la Guayana). El análisis de los cocolitos en los testigos estudiados (1240, 1241, 1242 y MD03-2616) forma parte de una serie de estudios que permitirán lograr los objetivos científicos de las campañas oceanográficas del "Ocean Drilling Program" (ODP) 202 y PICASSO llevadas a cabo en los océanos Pacífico oriental y Atlántico occidental respectivamente.

Los testigos fueron seleccionados con el fin de proporcionar información sobre la historia de la circulación oceánica superficial en los sectores tropicales estudiados. La interrelación de las cuencas de Panamá y de la Guayana localizadas en los sectores tropicales mencionados arriba respectivamente, facilitará una perspectiva regional de los patrones temporales y espaciales del registro de paleoproductividad y los mecanismos asociados a ella. Dichos mecanismos hacen referencia a los cambios estructurales de la zona eufótica, como la topografía de la nutritermoclina, y su relación con la dinámica hidrológica y atmosférica. La localización de los testigos en los sectores tropicales antes mencionados es muy apropiada para apreciar los cambios surnorte e inter-cuenca de paleoproductividad y de la topografía de la nutri-termoclina a ambos lados de Suramérica, asociados a la circulación oceánica superficial.

En esta Tesis, el estudio de la variabilidad de la dinámica de las masas de agua superficiales entre los dos sectores, está condicionado por la diferencia en resolución de estudio entre los testigos (entre 4 y 6 Ka para los testigos de la cuenca de Panamá, y 120 a 700 años para el de la cuenca de la Guayana). A lo anterior se le agrega el hecho de que el registro de cocolitos en la cuenca de Panamá llega hasta los $925 \mathrm{Ka}$, mientras que en la Guayana sólo alcanza el último ciclo climático (155 Ka). No obstante, la variabilidad milenaria de los perfiles de dichos testigos hace posible llevar a cabo una interpretación general del gradiente de productividad y de la variación general de la nutri-termoclina entre las cuencas de Panamá y de la Guayana durante los últimos $155 \mathrm{Ka}$.

Aunque la asociación de cocolitóforos es muy similar en los cuatro testigos, los factores que controlan la distribución temporal y espacial de los taxones, difieren entre las cuencas y 
dependen de la intensidad y sentido de los vientos alisios, los cambios en la posición latitudinal de la Zona de Convergencia Intertropical ("Intertropical Convergence Zone", ITCZ) y de los elementos atmosféricos e hidrográficos asociados a ellos. En este capítulo se explica la evolución del escenario paleoceanográfico y el sistema de circulación atmosférica durante el Pleistoceno medio y tardío en los sectores tropicales del océano Pacífico oriental y Atlántico occidental, la manera cómo se reflejan esos cambios paleoceanográficos en la asociación de cocolitóforos y la sensibilidad de esta asociación a los eventos análogos a "El Niño" a escala milenaria. Para este fin fue necesario introducir en este capítulo nuevos perfiles obtenidos en esta Tesis Doctoral y que no fueron incluidos en las publicaciones referidas en los capítulos 5, 6 y 7 . Estos perfiles corresponden a la tasa de acumulación del carbonato derivado de los cocolitos (CAR) para los testigos 1240 y MD03-2616, y a la tasa de acumulación de cocolitos (NAR) para los testigos 1241 y 1242. La incorporación de estos nuevos registros se realizó con el ánimo de facilitar las correlaciones entre los testigos. En el caso del testigo 1240, el producto de los valores NAR y del carbonato total aportado por los cocolitos previamente estimados en el capítulo 5, permitió la estimación de los valores CAR (capítulo 4). Para el testigo MD03-2616, los valores NAR (capítulo 7) fueron convertidos a valores CAR a través de su multiplicación por los valores del carbonato total de los cocolitos (capítulo 4). La reconstrucción del registro NAR para los testigos 1241 y 1242 se obtuvo a través de la división de los valores CAR entre el carbonato total de los cocolitos (capítulos 4 y 6 ).

\subsection{Cronología y bioestratigrafía}

\subsubsection{Océano Pacífico oriental y tropical}

Las secuencias estratigráficas estudiadas en los testigos del Pacífico 1240, 1241 y 1242 comprenden un rango de edad que va desde el Pleistoceno medio hasta el Holoceno. La estratigrafía de isótopos estables $\delta^{18} 0$, los biohorizontes de los cocolitos y el paleomagnetismo conforman un excelente conjunto de datos que respalda los puntos de control edad-profundidad para construir los modelos de edad en los diferentes testigos (capítulos 5 y 6 ).

Los isótopos estables fueron medidos en las conchas del foraminífero planctónico Globigerinoides ruber (blanco) en el testigo 1240 durante los últimos 329 Ka (Cacho et al., comunicación personal, 2006) y en el testigo 1242 durante los últimos 925 Ka (Benway y Mix, 2004; Mix et al., comunicación personal, 2005). La estratigrafía $\delta^{180}$ de ambos testigos se 
correlacionó gráficamente con la estratigrafía $\delta^{18} \mathrm{O}$ de G. ruber (blanco) del testigo 677 (Shackleton et al., 1990) localizado en el Océano Pacífico oriental tropical (capítulos 5, 6).

La excelente datación de toda la secuencia estudiada en el testigo 1242 fue utilizada como una referencia estratigráfica potencial para establecer el marco cronoestratigráfico del testigo 1241 junto con el evento Brunhes/Matuyama, ya que la estratigrafía $\delta^{18} O$ de este último testigo aún se encuentra en preparación. Por ende, la biocronología del testigo 1242 fue transferida a los eventos bioestratigráficos identificados en el testigo 1241. Adicionalmente, valiéndonos del patrón glacial/interglacial en el registro del carbonato en el Pacífico, así como del flujo del carbonato aportado por los cocolitos en el testigo 1242, se pudieron establecer puntos de control intermedios a los bioeventos en el testigo 1241, permitiendo así optimizar la cronoestratigrafía de este último testigo (capítulos 5 y 6 ).

La bioestratigrafía de los testigos del Pacífico fue documentada por Flores et al. (2006), a la que posteriormente, se le agregaron muestras adicionales localizadas estratégicamente para incrementar la resolución de los intervalos estudiados en cada testigo (capítulos 5 y 6) (Fig. 8.1).

Los siguientes biohorizontes de cocolitóforos del Pleistoceno fueron identificados en los testigos del Pacífico. El Último Registro ("Last Occurrence Datum" LOD) de Reticulofenestra asanoi (880 Ka; Raffi, 2002), el Primer Registro Común ("First Common Occurrence Datum”, FCOD) de Gephyrocapsa caribbeanica (el cual, de acuerdo con varios autores, se ha identificado entre los MIS 15 a 12, Flores et al., 1999; Bollman et al., 1998; Baumann y Freitag, 2004; Álvarez, 2005; entre otros), la LOD de Pseudoemiliania lacunosa (458 Ka; Thierstein et al., 1977), el Primer Registro ("First Occurrence Datum", FOD) de Emiliania huxleyi (268 Ka; Thierstein et al., 1977) el Último Registro Común ("Last Common Occurrence Datum”, LCOD) de G. caribbeanica (en el límite de los estadios isotópicos 8/7, Thierstein et al., 1977) (Capítulos 5 y 6).

La LOD de P. lacunosa y el evento paleomagnético Brunhes/Matuyama (Mix et al., 2003) fueron los puntos de control utilizados desde la base de la secuencia estudiada hasta los $329 \mathrm{Ka}$ en el testigo 1240, indicando que la máxima edad de estos sedimentos era inferior a $780 \mathrm{Ka}$. De igual manera, la LOD de R. asanoi en 880 Ka (Raffi, 2002) y el evento Brunhes/Matuyama (Mix et al., 2003) establecieron que la secuencia del testigo 1241 tiene una edad inferior a los $880 \mathrm{Ka}$ (Capítulos 5 y 6 ). 


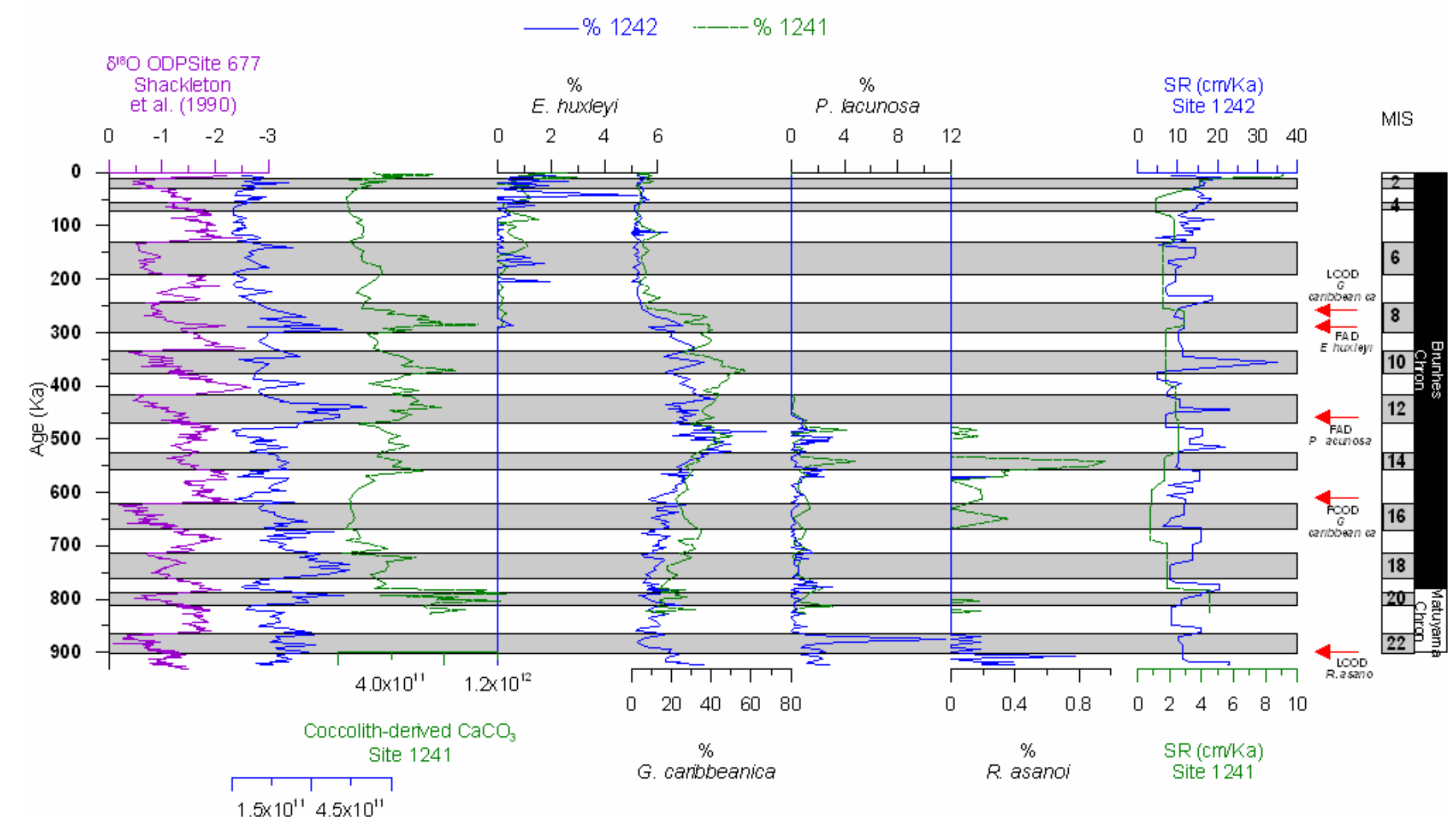

Coccolith-derived $\mathrm{CaCO}_{3}$

$$
\text { Site } 1242
$$

Fig. 8.1. Síntesis de la biocronología de los testigos 1240, 1241 y 1242 del océano Pacífico oriental. MIS: Estadio Isotópico Marino, "Marine Isotope Stage; MIS pares corresponden a los episodios glaciales y los MIS impares corresponden a los episodios interglaciales. LCOD: Último Registro Común ("Last Common Occurrence Datum'); FCOD: Primer Registro Común (“Frst Common Occurrence Datum"); FAD: Primer Registro (“Frst Appearance Datum"). 


\subsubsection{Océano Atántico occidental tropical}

El modelo de edad del testigo MD03-2616 está basado en una correlación gráfica de la estratigrafía isotópica del foraminífero bentónico Uvigerina peregrina del testigo con la curva de $\delta^{180}$ de foraminíferos bentónicos de Lisiecki y Raymo (2005) durante los últimos $155 \mathrm{Ka}$ (Capítulo 7).

El estudio bioestratigráfico de los microfósiles calcáreos del testigo MD03-2616, pone de manifiesto que la secuencia estudiada es más joven que $268 \mathrm{Ka}$. Este dato está avalado por el registro continuo aunque poco abundante de E. huxleyi y de la Biozona W de Ericsson y Wollin (1956). Los biohorizontes de los foraminiferos planctónicos identificados en el testigo de la cuenca de la Guayana mostraron una buena correlación con la Biozonación Climática descrita por Ericson y Wollin (1956) y Kenneth y Huddlestun (1972), la cual incluye la aparición, desaparición y cambios en la abundancia de los foraminíferos planctónicos del grupo de Globorotalia menardii y del taxón Pulleniatina obliquiloculata. Estas biozonas han sido utilizadas tradicionalmente como herramientas cronoestratigráficas en el océano Atlántico occidental y el mar Caribe, las cuales en nuestro testigo confirmaron la cronoestratigrafía de la secuencia (Figura 2).

Los principales eventos bioestratigráficos de los cocolitos durante los últimos $155 \mathrm{Ka}$, tales como el acme de E. huxleyi y la inversión de las abundancias relativas entre E. huxleyi y G. muellerae (Thierstein et al., 1977) no fueron identificados en la cuenca de la Guayana ni en la cuenca de Panamá. La baja abundancia relativa de E. huxleyi limitó la identificación de estos eventos, lo cual fue atribuido a las altas abundancias de G. oceanica o a los efectos de la temperatura superficial (Capítulos 5, 6 y 7).

\subsection{Presenvación}

La buena preservación del carbonato de los cocolitos en los testigos más profundos (1240 y 1241) apunta a que estos testigos estuvieron siempre por encima de la lisoclina (lo cual es especialmente significativo en el testigo 1240 localizado muy cerca de la lisoclina en el área de Galápagos) y a que no sufrieron procesos postsedimentarios que determinaran su alteración (Capítulos 5 y 6$)$. 
La variación de la disolución del carbonato del testigo 1242 pone de manifiesto una buena preservación durante los eventos glaciales y moderada preservación durante los interglaciales, siguiendo el patrón glacial/interglacial de la preservación del carbonato característica del océano Pacífico (Arrhenius, 1952; Archer, 1991a, b; Karlin et al., 1992; Farrell and Prell, 1991, Lyle et al., 2002; entre otros). Algunos intervalos del registro del testigo 1242 mostraron evidencias de una preservación muy deteriorada (el final del estadio 15, la mitad del estadio 13, el final del estadio 7 y el estadio 5, excepto el subestadio 5.3) enmascarando ligeramente el significado micropaleontológico del registro (Capítulo 6). Sin embargo, la preservación moderadamente buena del carbonato durante la mayor parte del testigo 1242 y los incrementos en la abundancia relativa de las especies más calcificadas $G$. oceanica, G. caribbeanica, C. leptoporus y $H$. carteri que no se vieron afectadas por la disolución selectiva de los cocolitos, indican que la señal micropaleontológica no se vio perturbada por la de disolución (Capítulo 6).

La preservación buena a moderada observada a lo largo de los testigos 1240 y 1241, y las diferencias en el contenido del carbonato de los cocolitos, que es más alto en los testigos $1240 \mathrm{y}$ 1241 y más bajo en el testigo 1242, sustenta la idea de que el carbonato de los cocolitos fue más propenso a la disolución cuando su producción fue más reducida (Capítulos 5 y 6 ).

El testigo MD03-2616 puso en evidencia una preservación general buena a moderada a lo largo de la secuencia estudiada, con excepción del final del subestadio 5.5 y todo el subestadio 5.4 , en los que la preservación se deterioró significativamente enmascarando el carácter micropaleontológico del registro durante esos eventos. El deterioro en la preservación durante los eventos 5.5 y 5.4 fue avalado por el desproporcionado incremento en las abundancias relativas de $G$. oceanica y $F$. profunda, especies relativamente resistentes a la disolución (Capítulo 7).

Basándonos en estas líneas de evidencia se puede afirmar que los registros de cocolitos indican una alta preservación en la zona de divergencia ecuatorial la cual se deteriora progresivamente hacia el norte de la cuenca de Panamá. Adicionalmente, se destaca el hecho de que en términos generales la preservación del carbonato es más elevada en la cuenca de Panamá respecto a la cuenca de la Guayana. La profundidad de los testigos 1242 y MD03-2616 indica que la disolución del carbonato en estos testigos está controlada por la degradación de la materia orgánica en el fondo del océano. 


\subsection{La variabilidad de la nutri-termoclina: el patrón glacial/interglacial en la cuenca de Panamá}

El registro temporal de la asociación de cocolitóforos muestra que la señal estuvo predominantemente dominada por cambios en la abundancia relativa de los habitantes de la zona fótica superior "small" Noelaerhabdaceae y G. oceanica. Las altas proporciones de $F$. profunda indican que esta especie fue también importante en términos cuantitativos a partir de los últimos $283 \mathrm{Ka}$ (estadio 8). En los cuatro testigos, el predominio general de los "small" Noelaerhabdaceae y G. oceanica sobre los habitantes de la zona fótica inferior representados por $F$. profunda, refleja un índice $\mathrm{N}$ relativamente alto y por ende, una paleoproductividad relativamente alta y una nutri-termoclina relativamente somera, con algunas oscilaciones (Capítulos 5 y 6; Fig. 8.2).

Las variaciones en el flujo total del carbonato aportado por los cocolitos (CAR), son paralelas al índice $\mathrm{N}$ en los testigos 1241 y 1242. Esto implica, que estos indicadores han respondido al mismo mecanismo oceanográfico/atmosférico, por lo que el registro del CAR fue utilizado también como un indicador de paleoproductividad. El CAR mostró máximos durante los estadios glaciales, lo cual es consistente con el patrón observado en el perfil típico del carbonato en el océano Pacífico. El registro del carbonato del Pacífico indica que la máxima producción de carbonato ocurrió durante los episodios glaciales (Arrenhius, 1952; Pedersen, 1983; Farrell y Prell, 1989; Archer, 1991a, b; entre otros). Por consiguiente, la paleoproductividad refleja un patrón glacial/interglacial en los testigos 1241 y 1242, con máximos durante los eventos glaciales (Capítulo 6).

Los cambios en la paleoproductividad a escala glacial/interglacial en la "warm pool" de la cuenca de Panamá han estado asociados a los cambios en la estructura de la capa de mezcla, que a su vez se deben a la intensificación o debilitamiento de la corriente Norecuatorial ("North Equatorial Countercurrent", NECC). Durante los eventos glaciales, se redujo la afluencia de aguas cálidas empujadas por la NECC hacia la cuenca de Panamá, favoreciendo la somerización de la nutritermoclina; sin embargo, durante los eventos interglaciales, se intensificó la advección de aguas cálidas arrastradas por la NECC haciendo que la capa de mezcla se hiciera más profunda y la nutri-termoclina descendiera (Capítulo 6). 


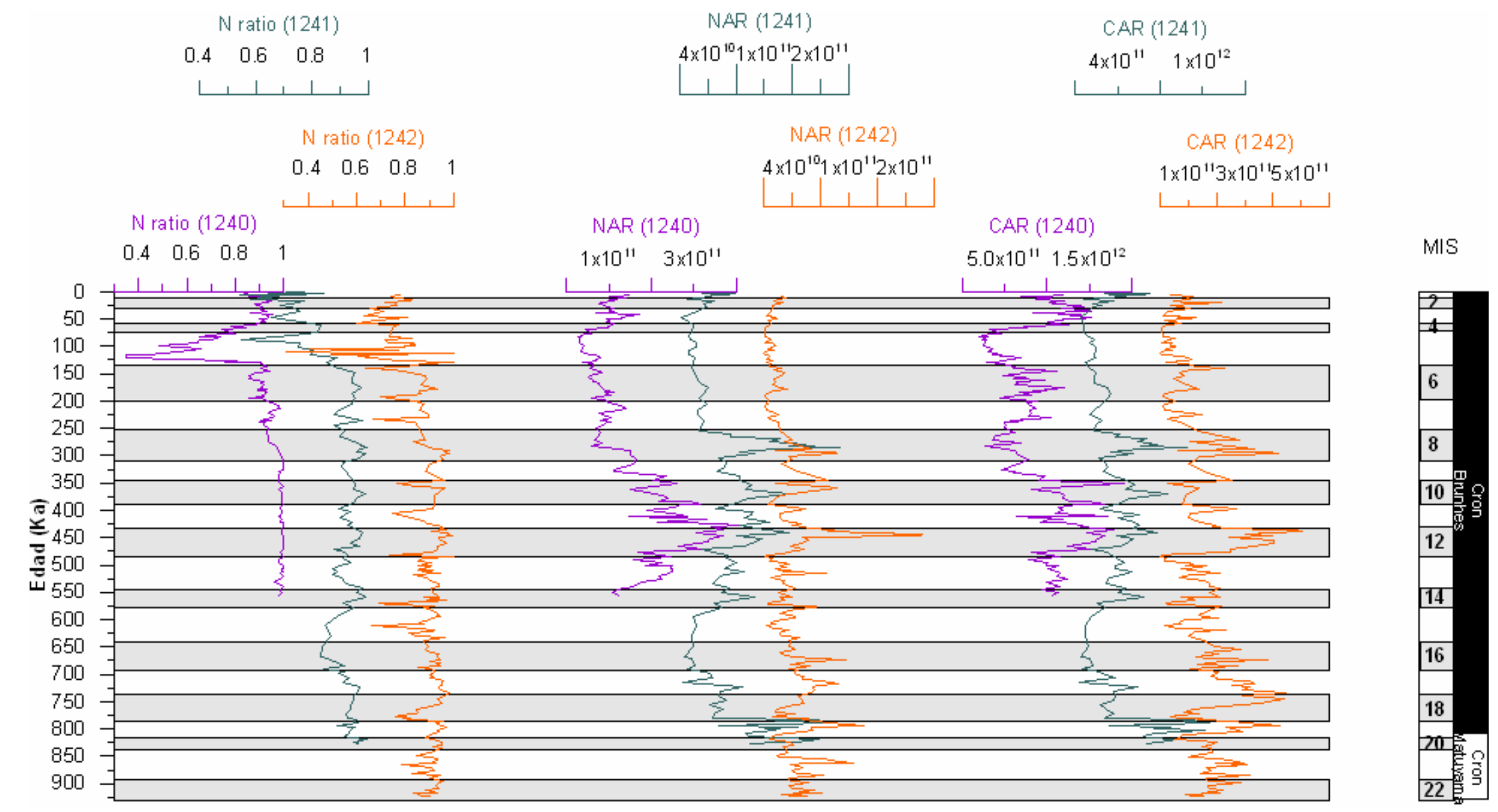

Fig. 8.2. Comparación de los registros indicadores de paleoproductividad (índice N; flujos de cocolitos $-\mathrm{NAR}$ - y flujo del carbonato de los cocolitos-CAR), para los testigos 1240, 1241 y 1242. NAR: cocolitos ${ }^{*} \mathrm{Cm}^{2 *} \mathrm{Ka}^{-1}$. CAR: $\mathrm{pg}^{*} \mathrm{Cm}^{2 *} \mathrm{Ka}^{-1}$. MIS: Estadio Isotópico Marino, "Marine Isotope Stage"; Los MIS pares corresponden a los episodios glaciales y los MIS impares corresponden a los episodios interglaciales. 
La variabilidad glacial/interglacial no fue evidente en los indicadores de paleoproductividad en el testigo 1240, sugiriendo que la ITCZ y el frente ecuatorial siempre permanecieron al norte del testigo 1240, lo cual permitió que la zona de divergencia ecuatorial mantuviera continuamente una célula de surgencia intensa para sostener la nutri-termoclina somera aunque con ligeras fluctuaciones. La permanente somerización de la nutri-termoclina también sugiere que la EUC se mantuviera somera y ésta, junto con la SEC, fueran lo suficientemente intensas (capítulo 5). Por ello, se podría concluir que la sensibilidad en la producción de los cocolitóforos en la zona de divergencia ecuatorial está relacionada principalmente con otras causas, no con la variabilidad glacial/interglacial, por ejemplo, con los eventos análogos a "El Niño"/"La Niña" a escala milenaria (capítulo 4).

En el Pacífico oriental tropical, los valores del índice $\mathrm{N}$ del testigo 1240 son los más altos y significativamente más constantes que en los demás testigos del Pacífico oriental. Por ejemplo, la paleoproductividad máxima ocurrió en la zona de divergencia y fue disminuyendo paulatinamente hacia el norte del Pacífico oriental tropical. Esto indica que la paleoproductividad revela un patrón sur-norte en el Pacífico oriental tropical durante el Pleistoceno medio y todo el Pleistoceno tardío (capítulos 5 y 6). Este cambio de la paleoproductividad también se observa entre el Pacífico oriental y Atlántico occidental tropicales, y es el resultado de las diferencias en la profundidad de la nutri-termoclina en las localidades de los testigos, poniendo de manifiesto una nutri-termoclina más elevada en el testigo 1240 y por lo tanto, una tasa de surgencia de aguas y de disponibilidad de nutrientes superior en este testigo que las de los otros testigos. Todo ello vinculado a la influencia directa de una de las áreas de surgencia de aguas más intensa en la zona de divergencia ecuatorial. De esta manera, la disponibilidad de aguas ricas en nutrientes se redujo hacia el norte de la cuenca de Panamá; y en la cuenca de la Guayana respecto a la cuenca de Panamá. Estas diferencias en la posición de la nutri-termoclina y la disponibilidad de nutrientes en la zona fótica superior son concordantes con la posición media anual de la nutri-termoclina en la actualidad. La profundidad actual de la nutri-termoclina en la zona de divergencia es de $\sim 40 \mathrm{~m}$, en la "warm pool" es de $\sim 50 \mathrm{~m}$ (Fiedler, 2002) y en la cuenca de la Guayana es de $\sim 150$ m (Pailler et al., 1999), la cual también revela un gradiente general sur-norte en la Cuenca de Panamá y un gradiente general entre los océanos Pacífico oriental y Atlántico occidental tropicales. 


\section{5. ¿Eventos análogos a “G Niño" a escala milenaria?}

El patrón general del índice $\mathrm{N}$ en los testigos 1240, 1241 y 1242 revela tres intervalos comunes de paleoproductividad (Capítulos 5 y 6; Fig. 8.2):

El primero, caracterizado por los más altos valores de paleoproductividad, comienza en la base de las secuencias estudiadas (MIS $14-560 \mathrm{Ka}$; MIS 20 -830 Ka y MIS 22 -925 Ka; para los testigos 1240, 1241 y 1242 respectivamente) y se extiende hasta el MIS 6 (128 Ka). Sin embargo, este intervalo muestra una ligera reducción de paleoproductividad desde comienzos del MIS 8 (283 Ka) hasta MIS 6 (128 Ka), aunque ésta sigue siendo relativamente alta y puede ser comparable a la del MIS 22 al 8 (830 Ka a $283 \mathrm{Ka}$ ). La reducción de la paleoproductividad desde los 283 Ka en la cuenca de Panamá coincidió con la reducción en la abundancia de G. caribbeanica, especie fuertemente calcificada, habitante de la zona fótica superior. Esta especie fue dominante en términos de abundancia y del carbonato aportado durante el período del Evento del "mid"-Brunhes (MIS 15 al 8; $615 \mathrm{Ka}$ a $283 \mathrm{Ka}$ respectivamente). De esta manera la alta paleoproductividad observada durante el período comprendido entre la base de las secuencias estudiadas y el MIS 6 es congruente con un período dominado por una intensificación generalizada de la surgencia de aguas frías y ricas en nutrientes desde las profundidades intermedias, lo cual mantuvo la nutri-termoclina siempre muy superficial en la cuenca de Panamá, situación muy similar a las condiciones de "La Niña" con algunas fluctuaciones.

El segundo intervalo coincide con el más fuerte incremento en la abundancia relativa de $F$. profunda y por consiguiente una reducción marcada de los valores de paleoproductividad durante el MIS 5 (128 Ka a $71 \mathrm{Ka}$ ). La variación de la paleoproductividad durante el MIS 5 se debió probablemente a una fuerte estratificación del océano superficial, con la consiguiente reducción del contenido promedio de nutrientes en la zona fótica superior en la cuenca de Panamá. Por consiguiente, este MIS se relaciona con un evento análogo al evento "El Niño" actual, debido al colapso de la célula de surgencia de aguas, y la subsecuencte profundización de la nutri-termoclina, que afectó no solo la zona de divergencia ecuatorial, sino a todo el sector que está fuera de ella en el Pacífico oriental tropical norte (capítulos 5 y 6). Lea et al. (2006) afirmó que las más altas temperaturas superficiales del océano en el área de Galápagos ocurrieron en el subestadio 5 e y luego fueron decreciendo gradualmente hasta el subestadio $5 \mathrm{a}$, 
lo cual es consistente con la variabilidad de la nutri-termoclina observada en el testigo 1240. Este patrón característico en la variación de la paleoproductividad indica que las propiedades de las masas de agua del océano superficial son comparables en los tres testigos del Pacífico durante los intervalos 1 y 2 respectivamente.

El último intervalo comprende los MIS 4 al 1 (últimos $71 \mathrm{Ka}$ ), muestra una tendencia hacia el aumento de la paleoproductividad en el testigo 1240; mientras que en los testigos 1241 y 1242 estos valores siguen siendo bajos y comparables con aquellos observados durante el MIS 5, con algunas fluctuaciones. El registro de productividad durante el período del MIS 4 al 1 indica que la nutri-termoclina en la zona de divergencia ecuatorial volvió a somerizarse, aunque en menor medida si se compara con el intervalo previo al MIS 5 y por lo tanto, el incremento en la paleoproductividad en la zona de divergencia ecuatorial, puede correlacionarse con un evento análogo a "La Niña" con algunas oscilaciones. A pesar de ello, la productividad en la "warm pool" no se recuperó durante los MIS 4 al 1, sugiriendo que aunque los vientos alisios se intensificaron tal como lo indica el aumento en la paleoproductividad en la zona de divergencia ecuatorial, éstos no fueron lo suficientemente intensos como para somerizar igualmente la nutri-termoclina en la "warm pool", tal como sucedió durante el primer intervalo (MIS 22 al 6).

\subsection{Influencia de la insolación en la posición de la nutri- termoc lina en el Atlántico occidental tropical}

La alta resolución del estudio de la asociación cocolitoforal en el Atlántico occidental tropical permitió el análisis de los taxones y de la paleoceanografía superficial en esta cuenca a escala centenial. La asociación nanofósil está representada principalmente por la alternancia en la abundancia de "small" Noelaerhabdaceae frente a la especie F. profunda, mientras que la abundancia de G. oceanica fluctúa a un nivel intermedio entre las primeras especies. Estos taxones son los más importantes en términos cuantitativos de la asociación. La alternancia y los cambios en estas especies permitieron la estimación del índice de paleoproductividad (índice N) y la tasa de acumulación de cocolitos ("Nannofossil Accumulation Rate"-NAR; Fig. 8.3). La comparación de estos indicadores sugiere un paralelismo en su variabilidad, lo cual indica que la tasa de acumulación de cocolitos es una buena imagen de lo que fue la producción en las masas de agua superficiales de la cuenca durante los últimos $155 \mathrm{Ka}$. La fluctuación en la producción en esta zona estuvo controlada por la migración vertical de la nutri-termoclina, que fue a su vez 
reflejo de la intensidad de la surgencia costera. La variabilidad en la paleoproductividad también sugiere que el período estudiado puede dividirse en dos intervalos (Capítulo 7).

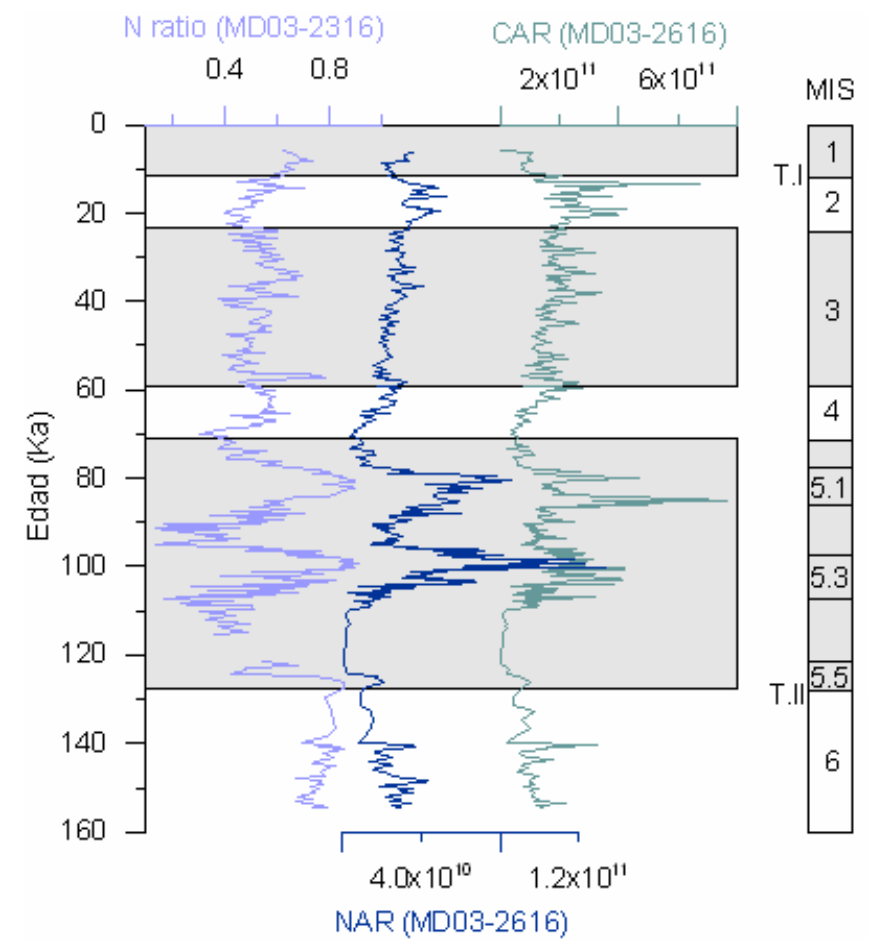

Fig. 8.3. Comparación de los registros indicadores de paleoproductividad (índice $\mathrm{N}$, flujos de cocolitos -NAR- y flujo del carbonato de los cocolitos-CAR), para el testigo MD03-2616. T.I y T.II: Terminaciones I y II. MIS: Estadio Isotópico Marino, "Marine Isotope Stage; MIS pares corresponden a los episodios glaciales y los MIS impares corresponden a los episodios interglaciales. 5.1, 5.2, 5.3, subestadios interglaciales del MIS 5. NAR: cocolitos ${ }^{*} \mathrm{~cm}^{-2 *} \mathrm{Ka}^{-1}$. CAR: $\mathrm{pg}^{*} \mathrm{Cm}^{-2^{*}} \mathrm{Ka}^{-1}$.

El primer intervalo se extiende desde el MIS 6 hasta el MIS 5 y refleja una variabilidad con fuertes cambios de paleoproductividad, producidos por oscilaciones en la dinámica de la circulación del océano superficial, regulados por la intensidad y dirección de los vientos alisios. Se ha registrado un máximo de paleoproductividad durante los subestadios interglaciales del MIS 5 como respuesta a una somerización de la nutri-termoclina, debida a la intensificación de la surgencia costera en la cuenca de la Guayana y a la dirección de los vientos alisios que soplaban paralelamente a la costa. Esto permitió que la masa de agua cálida de la NEC se desplazara hacia el Atlántico oriental debido a una intensificación de la NECC. Durante los subestadios glaciales, esta relación hidrológico-atmosférica cambió, debido a una intensificación de los vientos alisios del noreste sobre los del sureste, debilitando la célula de surgencia costera y la NECC, favoreciendo con ello el apilamiento de aguas cálidas traídas por la NEC desde el Atlántico oriental.

El segundo intervalo se extiende desde el MIS 4 hasta el Holoceno. La asociación de cocolitóforos está constituida principalmente por una alta abundancia relativa de F. profunda, comparable o ligeramente inferior a la de los "small" Noelaerhabdaceae y G. oceanica. Este 
intervalo se caracteriza por valores de paleoproductividad más bajos y más constantes que los del intervalo previo, indicando que las variaciones en la posición de la nutri-termoclina fueron más moderadas. Todo ello indica que la célula de surgencia costera se debilitó en la cuenca de la Guayana debido a su desplazamiento hacia el sur siguiendo la migración latitudinal de la ITCZ como consecuencia de la intensificación de los vientos alisios del noreste. Estos vientos soplaron perpendicularmente a la costa causando la acumulación de una masa de agua cálida arrastrada por la NEC y el debilitamiento de la NECC. Sin embargo, algunos picos de paleoproductividad coinciden con los substadios interglaciales 3.3, 3.1 y el comienzo del MIS 1 (Holoceno), poniendo de manifiesto una activación gradual de la surgencia costera en la cuenca de la Guayana durante esos episodios.

La variabilidad de la paleoproductividad refleja una relación notablemente consistente con la curva de insolación de verano en el Hemisferio Norte $(65 \circ \mathrm{N})$ sugiriendo máximos de paleoproductividad durante los máximos de insolación a 65N. La variabilidad de la insolación a $65^{\circ} \mathrm{N}$ ejerció una influencia directa en los cambios de volumen del hielo en el Hemisferio Norte, lo cual tuvo a su vez un impacto importante en la intensidad de los vientos alisios del noreste y por consiguiente en la migración latitudinal de la ITCZ y en la circulación oceánica (Hastenrath y Greischar, 1993; Hastenrath y Druyan, 1993; Khodri et al., 2003). De esta manera la variabilidad de la paleoproductividad y por tanto en la migración vertical de la nutri-termoclina, revelan una conexión entre las altas latitudes del norte del océano Atlántico y las bajas latitudes del mismo océano.

La cuenca de la Guayana ha estado asociada al patrón de drenaje de los ríos Amazonas y Orinoco, disminuyendo la salinidad en ese sector del océano Atlántico occidental durante los períodos en los que la ITCZ se localiza en su posición norte (Showers y Bevis et al., 1988; Pailler et al., 1999; Masson y Delecluse, 2001). La posición norte de la ITCZ también favorece el aumento del influjo de sedimentos terrígenos (Pailler et al., 1999; Pujos y Froidefond, 1995), la llegada masiva de nutrientes provenientes del continente (Peterson et al., 2000; Vink et al., 2001; Martínez et al., 2007) y con ello el aumento en la concentración de clorofila en el océano (SeaWIFS Project Imagery; http://oceancolor.gsfc.nasa.gov/SeaWiFS). No obstante, la asociación de cocolitóforos no revela evidencias de cambios en la influencia de agua dulce en el océano superficial, aunque éstas no se descartan (Capítulo 7). 
Aunque el índice $\mathrm{N}$ del testigo del Atlántico occidental tropical registre un patrón completamente diferente al del Pacífico oriental tropical, la evolución de la productividad puso de manifiesto un fuerte gradiente en el índice $\mathrm{N}$ entre ambos sectores durante el último ciclo climático (capítulos 5, 6 y 7). Asimismo, el índice $N$ se vio disminuido en el testigo MD03-2616 en relación a los índices en los testigos del Pacífico, demostrando que la paleoproductividad en el Atlántico occidental tropical es inferior a la del Pacífico oriental tropical durante la mayor parte del último ciclo climático, con excepción de los subestadios 5.3 y 5.1 , en donde la nutri-termoclina fue especialmente somera en el Atlántico occidental tropical, mientras que se profundizaba en el Pacífico oriental tropical (Fig. 8.2 y 8.3).

\subsection{Consideraciones finales}

Los océanos Pacífico y Atlántico tropicales comparten características climatológicas comunes tales como la dinámica de los vientos alisios, la somerización de la nutri-termoclina en el sector oriental de estos océanos, la zona de divergencia ecuatorial y la situación norte de la ITCZ. No obstante, al comparar la variabilidad climática entre estos dos sectores tropicales, se revelan más diferencias que similitudes (Xie et al., 1999), teniendo en cuenta que ambos sectores se localizan en las regiones oriental y occidental (del Pacífico y Atlántico respectivamente), las cuales presentan dinámicas opuestas, tal como lo indica la dinámica oceanográfica actual. El Pacífico está dominado por el fenómeno de ENSO mientras la ITCZ en el Atlántico está controlada por cambios interhemisféricos en los gradientes de las temperaturas superficiales del océano ("sea surface temperatures", SST). Un evento "El Niño" en el Pacífico puede desplazar la ITCZ hacia el ecuador, mientras que en el Atlántico la amplitud del gradiente de las SST se debilita muy poco como para afectar la posición de la ITCZ (Servain et al., 1999). El desplazamiento sur-norte de la ITCZ en el Atlántico es más pronunciado durante la primavera boreal, cuando ocurre el aumento estacional de las temperaturas en el ecuador. De acuerdo con ésto, la variabilidad de la ITCZ y la dinámica atmosférica a gran escala, provocan una reorganización de la variabilidad climática tanto en los sectores tropicales como en los extratropicales (Xie et al., 1999).

Las investigaciones realizadas en esta Tesis Doctoral han puesto de manifiesto un evento análogo a "La Niña" persistente durante los MIS 22 al 6 (925 Ka a $128 \mathrm{Ka}$ ) y 4 al 1 (últimos 71 Ka), y un evento análogo a "El Niño" durante el MIS 5 (128 Ka a $71 \mathrm{Ka}$ ) en el Pacífico oriental. El 
Atlántico occidental tropical en el MIS 5 por ejemplo, experimentaba los más fuertes cambios de paleoproductividad y de somerización/profundización de la nutri-termoclina. Sin embargo, las diferencias de resolución y de longitud temporal de los testigos estudiados en esta Tesis no revela evidencias de una conexión interocéanica entre los sectores tropicales estudiados durante un evento "El Niño", aunque no se descarta la influencia de los eventos ENSO del Pacífico sobre el Atlántico tropical.

Esta hipótesis puede ser abordada incrementando la resolución de muestreo en todos los testigos (centenial a ser posible), y aumentando el intervalo temporal estudiado al menos para los últimos $925 \mathrm{Ka}$ en los testigos 1240, 1241 y MD03-2616 con el fin de establecer comparaciones de carácter micropaleontológico (nanoplancton calcáreo) y geoquímico (isótopos estables, elementos traza y alquenonas) entre todos los testigos a la misma escala y para los mismos ciclos climáticos. La correlación entre los diferentes indicadores permitiría un análisis comparativo entre los cambios en la productividad y las fluctuaciones de la nutri-termoclina reveladas por la asociación florística y aquellos revelados por otros indicadores geoquímicos, como las alquenonas y los elementos traza e isótopos estables en foraminíferos planctónicos. $\mathrm{E}$ análisis geoquímico de los foraminíferos bentónicos permitiría establecer una relación entre la dinámica superficial y profunda (en ambos sectores tropicales influidos por la AAIW) y su vínculo con la circulación termohalina, tal como explicó Pena (2008) para el Pacífico oriental ecuatorial para los últimos $250 \mathrm{Ka}$.

\section{Apéndice: Consideraciones paleoecológicas de la asoc iación cocolitoforal}

La asociación está representada principalmente por ciertas especies de la Familia Noelaerhabdaceae, las cuales corresponden a los géneros Gephyrocapsa, Reticulofenestra, Emiliania and Pseudoemiliania (Thierstein et al., 1977; Pujos-Lamy, 1977; Wei, 1993; Raffi et al., 1993). La clasificación de los individuos de esta Familia está basada en una subdivisión morfométrica, así por ejemplo, los taxones de tallas superiores a las $3 \mu \mathrm{m}$ incluyen Gephyrocapsa oceanica, Gephyrocapsa muellerae, Reticulofenestra asanoi y Pseudoemiliania lacunosa. Los individuos de tallas inferiores a las $3 \mu \mathrm{m}$, llamados comúnmente "small" Noelaerhabdaceae, están representados por Emiliania huxleyi y Gephyrocapsa ericsonii, y también incluyen Gephyrocapsa aperta, la cual está caracterizada por un área central cerrada y 
una talla inferior a las $2.5 \mu \mathrm{m}$. De esta manera, aquellos gephyrocapsidos que tienen un área central cerrada y una talla superior a las $2.5 \mu \mathrm{m}$ fueron identificados como Gephyrocapsa caribbeanica (Apéndices A y B). Florisphaera profunda también forma parte de las especies dominantes dentro de la asociación, mientras otros taxones como Calcidiscus leptoporus, Helicosphaera carteri, "warm taxa" y Coccolithus pelagicus ocupan un lugar secundario.

"Small" Noelaerhabdaceae representan parte de los taxones que dominan la asociación porque registra abundancias muy altas. Su abundancia está controlada de manera significativa por los gephyrocapsidos, mas que E. huxleyi. Diversos autores han citado altísimas concentraciones en la abundancia de E. huxleyi en el océano global a partir de los últimos $73 \mathrm{Ka}$ (Thierstein et al., 1977), sin embargo en los sectores estudiados, su abundancia estaría limitada por la temperatura superficial de las masas de agua y/o por la altísima concentración de $G$. oceanica. La abundancia de los "small" Noelaerhabdaceae define extraordinariamente los períodos de intensificación de las células de surgencia en los testigos del Pacífico oriental y Atlántico occidental. Los "small" Noelaerhabdaceae han sido considerados como unos excelentes indicadores de células de surgencia en el océano (Gartner, 1988; Okada y Wells, 1997; Takahashi y Okada, 2000), masas de agua ricas en nutrientes (Gartner et al., 1987; Gartner, 1988; Takahashi y Okada, 2000; Flores et al., 2005) y descensos en la temperatura de las masas de agua eutróficas (Gartner, 1988) y por ello se les ha incluido dentro del grupo de estrategas de la R (Gartner et al., 1987; Gartner, 1988; Okada y Wells, 1997), aunque en esta investigación, el incremento en sus abundancias no supuso un cambio en la diversidad.

Gephyrocapsa oceanica fue separada en dos grupos morfométricos por motivos de estimación del carbonato derivado de los cocolitos (3-4 $\mu \mathrm{m}$ y $4-5 \mu \mathrm{m}$ ), siendo los dos morfotipos igualmente frecuentes dentro de la asociación. Al igual que los "small" Noelaerhabdaceae, G. oceanica también fue considerada como un taxón indicador de la célula de surgencia en los sectores tropicales estudiados. G. oceanica ha caracterizado áreas de surgencia en bajas latitudes (Mitchell-Innes y Winter, 1987; Ziveri et al., 1995; Broerse et al., 2000; Andruleit et al., 2000); otras masas de agua ricas en nutrientes (Winter, 1982; Mitchell-Innes y Winter, 1987; Gartner, 1988; Houghton y Guptha, 1991; Henriksson, 2000; Kinkel et al., 2000; Cortes et al., 2001; Andruleit y Rogalla, 2002; Böeckel y Baumann, 2004). También ha mostrado preferencias por masas de agua poco salinas (Tanaka, 1991; Klejine, 1993; Knappertsbusch, 1993; Jordan y Winter, 2000; Di Stefano e Incarbona, 2004), aguas cálidas (Okada y Honjo, 1973; Honjo y Okada, 1974; Geitzenauer et al., 1976; Thierstein et al., 1977; Okada y McIntyre, 1979; Gartner, 
1988; Kleijne et al., 1989; Giraudeau, 1992; Jordan y Chamberlain, 1997; Flores et al., 1999; Jordan y Winter, 2000; Hagino et al., 2000; Findlay y Giraudeau, 2000; Sprovieri et al., 2003; Di Stefano e Incarbona, 2004) o empobrecidas en nutrientes (Hagino et al., 2000).

Gephyrocapsa caribbeanica caracteriza de manera especial el Evento "mid"-Brunhes por su altísima concentración en número de individuos y en el carbonato aportado por ella. $G$. caribbeanica está asociada a una alta nutri-termoclina y una alta concentración del carbonato total aportado por los cocolitos en el océano Pacífico oriental tropical, revelando así un fuerte patrón paleoecológico. Varios autores han sugerido que su máxima concentración durante el Evento "mid"-Brunhes se debe a un patrón paleoecológico indicador de aguas eutróficas (Álvarez, 2005; Liu et al., 2008) o de aguas oligotróficas (Bollman et al., 1998), mientras que otros han hecho referencia a un patrón evolutivo (Bollman et al., 1998; Flores et al., 1999).

Florisphaera profunda reveló un patrón relacionado con masas de agua superficiales empobrecidas en nutrientes en las cuencas de Panamá y de la Guayana tal como lo demuestra el patrón inverso de sus abundancias relativas frente a las de los "small" Noelaerhabdaceae y G. oceanica. Esta especie ha sido citada como un habitante usual de la zona fótica inferior (Okada y Honjo, 1973; Okada y Mclntyre, 1977). La fluctuación de su abundancia relativa indica cambios en la producción de la zona fótica superior y en la migración vertical de la nutri-termoclina (Molfino y McIntyre, 1990a, b; Castradori, 1993; Jordan et al., 1996; Beaufort et al., 1997; Ziveri y Thunell, 2000). La relación de su abundancia relativa respecto a la de los "small" Noelaerhabdaceae y $G$. oceanica han permitido caracterizar las fluctuaciones de la paleoproductividad y de la nutri-termoclina durante el Pleistoceno medio y tardío, tal como ha sido previamente citado por varios autores (Beaufort et al., 1997; Takahashi y Okada, 2000; Flores et al., 2000; Álvarez, 2005; entre otros). Otros investigadores han correlacionado su alta abundancia con la transparencia de las aguas superficiales en el océano Pacífico noroccidental (Ahagon et al., 1993).

Gephyrocapsa muellerae revela un máximo aumento en sus proporciones (siempre por debajo del 20\%) en el testigo MD03-2616 durante los estadios fríos 4 al 2, caracterizados por un ambiente mesotrófico. En el resto de los testigos su tendencia no es clara en la asociación y además registra bajas abundancias, lo cual puede estar condicionado por la temperatura superficial de las masas de agua al igual que E. huxleyi, ya que G. muellerae ha sido descrita en 
ambientes de aguas relativamente frías (Weaver y Pujol, 1988; Bollmann, 1997; Flores et al., 1999, 2003).

Pseudoemiliania lacunosa está asociada a un evento fuertemente eutrófico entre los $925 \mathrm{Ka}$ y 458 Ka en la cuenca de Panamá. Varios autores han destacado su afinidad por ambientes con alta variabilidad en las condiciones del flujo de nutrientes y de salinidad (Marino et al., 2008).

Reticulofenestra asanoi corresponde a cocolitos mayores de $6.5 \mu \mathrm{m}$, subcirculares 0 elítpicos. Su última aparición ("Last Appeareance Datum”, LAD) datada los 880 Ka (Raffi, 2002) sólo fue registrada en el testigo 1242, debido a que las secuencias sedimentarias estudiadas en los testigos 1240, 1241 y MD03-2616 únicamente alcanzaron los últimos $560 \mathrm{Ka}, 830 \mathrm{Ka}$ y $155 \mathrm{Ka}$, respectivamente. Algunos trabajos han caracterizado la afinidad de $R$. asanoi por aguas de salinidad normal (Maiorano y Marino, 2004).

Calcidiscus leptoporus mostró afinidades por un ambiente más eutrófico en la "warm pool" del océano Pacífico oriental y en el Atlántico occidental tropicales, donde el flujo de nutrientes no es continuo en el océano superficial y depende de la intensidad y dirección de los vientos alisios. Mientras que en la zona de divergencia ecuatorial, caracterizada por un flujo continuo de nutrientes en la zona eufótica, esta especie no mostró un patrón paleoecológico característico. $C$. leptoporus ha sido caracterizado por su afinidad a ambientes eutróficos (Roth y Berger, 1975; Fincham y Winter, 1989; Andruleit y Rogalla, 2002; Flores et al., 2003), a ambientes oligotróficos tropicales a subtropicales y a las masas de agua correspondientes a la zona fótica superior y media (Mclntyre y Bè, 1967; Mclntyre et al., 1970; Okada y Honjo, 1973; Blasco et al., 1980; Klejine, 1993; Winter et al., 1994; Flores et al., 1999; Baumann et al., 2004; Ziveri et al., 2004; Marino et al., 2008). Esta especie ha sido clasificada en varios grupos (Lohmann, 1920; Knappertsbusch et al., 1997; Baumann y Sprengel, 2000; Quinn et al., 2004; entre otros) basados en esquemas relacionados con el diámetro del cocolito, en el número de elementos y/o el diámetro de la cocosfera. En esta tesis, $C$. leptoporus fue separado morfométricamente en los tres grupos de Knappertsbusch et al. (1997), para establecer una mejor aproximación en las estimaciones del carbonato aportado por los cocolitos, siendo el morfotipo intermedio (5-8 $\mu \mathrm{m})$ el más común en las observaciones realizadas. Sin embargo, el carácter paleoecológico de cada uno de estos morfotipos no es claro en los registros y además no se pudo diferenciar otro rasgo morfológico independiente del tamaño, así que se consideró más pertinente agrupar los tres morfotipos en una sola especie como Calcidiscus leptoporus s.l. 
Helicosphaera carteri mostró preferencias por masas de agua fértiles en áreas donde el flujo de nutrientes está influido por la intensidad y dirección de los vientos alisios. El flujo de esta especie se incrementó cuando la nutri-termoclina se hizo más somera en la "warm pool" de la cuenca de Panamá y en la cuenca de la Guayana. Sin embargo en la zona de divergencia ecuatorial donde el flujo de nutrientes es constante, su registro no es claro. $H$. carteri ha sido registrada durante períodos de alta paleoproductividad (Flores et al., 1995), en zonas de surgencia (Estrada, 1978; Giraudeau, 1992), en ambientes mesotróficos (Ziveri et al., 1995; Ziveri et al., 2000; Andruleit y Rogalla, 2002; Findlay y Giraudeau, 2002; Ziveri et al., 2004; Baumann et al., 2005), en ambientes oligotróficos (Haidar y Thierstein, 1997; Giunta et al., 2003), en masas de agua cálidas (McIntyre y Bè, 1967; Gard y Backman, 1990; Brand, 1994; Haidar y Thierstein, 1997; Baumann et al., 2005), en sapropeles (Müller, 1985; Castradori, 1993; Negri et al., 1999; Negri y Giunta, 2001; Negri et al., 2003). Esta especie igualmente fue separada de acuerdo a su morfometría en tres grupos (Knappertsbusch et al., 1997) por motivos de las estimaciones del carbonato aportado por los cocolitos, siendo el morfotipo intermedio (5-8 $\mu \mathrm{m})$ y el grande $(>8 \mu \mathrm{m})$ los más comunes en los registros de los cocolitos. Sin embargo, para el análisis de la señal paleoecológica, se ha creído más oportuno seguir el criterio clásico de considerar los tres morfotipos como una sola especie, Helicosphaera carteri s.l.

Coccolithus pelagicus ha sido relacionado con el ambiente eutrófico que registró la mínima abundancia de $F$. profunda y la máxima somerización de la nutri-thermoclina durante el Pleistoceno medio en los testigos 1240 y 1242 (desde $925 \mathrm{Ka}$ hasta $500 \mathrm{Ka}$ ). No obstante, su bajísima abundancia a lo largo de estos dos testigos y su ausencia en los testigos 1241 y MD032616 ha permitido concluir que este taxón está fuera de la zona paleoecológica correspondiente, la cual está estrechamente vinculada a la temperatura superficial del océano. Diversos autores han explicado las afinidades de esta especie con las masas de agua de las células de surgencia y otras masas de agua enriquecidas en nutrientes (Blasco et al., 1980; Giraudeau y Baley, 1995; Cachao y Moita, 2000; Boeckel y Baumann, 2004). Esta especie fue identificada en tres tamaños: 6-9 $\mu \mathrm{m}, 10-13 \mu \mathrm{m}$ and 14-16 $\mu \mathrm{m}$ (Baumann, 1995; Baumann et al., 2000; Cachão y Moita, 2000; Parente et al., 2004; Narciso et al., 2006) por motivos de las estimaciones del carbonato aportado por los cocolitos, siendo el morfotipo pequeño $(6-9 \mu \mathrm{m})$ el más común en la asociación a pesar de su baja abundancia relativa. Para el análisis paleoecológico, se consideró más pertinente agrupar los tres morfotipos en una sola especie como Coccolithus pelagicus s.l. 
"Warm taxa" es un grupo de especies representado principalmente por Umbilicosphaera sibogae. También se incluyen aunque en muy bajas abundancias a U. foliosa, U. hulburtiana, Oolithotus spp., Pontosphaera spp., Umbellosphaera spp., Neosphaera coccolithomorpha, Syracosphaera spp., Rhabdosphaera clavigera y Calciosolenia murrayi. Este grupo está caracterizado por sus preferencias por masas de agua cálidas y oligotróficas en el testigo 1240. Varios autores han indicado igualmente las afinidades de este grupo por un ambiente oligotrófico y de temperaturas superficiales relativamente altas (Hiramatsu and De Deckker, 1997; Böeckel y Baumann, 2004; Marino et al., 2008). En los testigos de la 1241, 1242 y MD03-3626, el comportamiento paleoecológico de $U$. sibogae mereció ser analizado separadamente del resto del grupo, indicando su afinidad por un ambiente eutrófico; mientras que en el testigo 1240 estuvo relacionada con un patrón más oligotrófico y aguas relativamente cálidas. Roth y Berger (1975), Roth y Coulbourn (1982), Andruleit et al. (2000), Flores et al. (2003) indicaron una relación positiva entre los picos en la abundancia de $U$. sibogae y aguas fértiles. Esta especie también ha caracterizado masas de aguas oligotróficas y relativamente cálidas (Okada y Mclntyre, 1979; Roth, 1994; Young, 1994; Böeckel y Baumann, 2004; Ziveri et al., 2004), y masas de aguasrelativamente salinas y eutróficas (Winter, 1982; Mitchell-Innes y Winter, 1987). Los demás taxones de este grupo no revelaron algún comportamiento característico a escala milenial posiblemente debido a su bajísima abundancia. Sin embargo, Syracosphaera spp. también ha sido citada como especie indicadora de aguas ricas en nutrientes (Estrada, 1978; Giraudeau, 1992) y en aguas relativamente poco salinas (Weaver and Pujol, 1988; Flores et al., 1997; Colmenero-Hidalgo et al., 2004).

\section{Referencias}

Ahagon, N., Tananka, Y., Ujiié, H. 1993. Florisphaera profunda, a possible nannoplankton indicator of Late Quaternary changes in sea-water turbidity at the northwestern margin of the Pacific. Marine Micropaleontology 22, 255-273.

Alvarez, M.C. 2005. Reconstrucción paleoceanográfica del margen continental del Pacífico durante los últimos 800 Ka mediante el análisis de asociaciones de cocolitóforos. Ph. D Thesis, Universidad de Salamanca, Salamanca, Spain, $151 \mathrm{pp}$.

Andruleit, H.A., von Rad, U., Bruns, A., Ittekkot, V. 2000. Coccolithophores fluxes from sediment traps in the north eastern Arabian Sea off Pakistan. Marine Micropaleontology 38, 285308.

Andruleit, H.A., Rogalla, U. 2002. Coccolithophores in surface sediments of the Arabian Sea in relation to environmental gradients in Surface waters. Marine Geology 186, 505-526.

Archer, D.E. 1991a. Equatorial Pacific calcite preservation cycles: production or dissolution?. Paleoceanography 6, 561-571.

Archer, D.E. 1991b. Modelling the calcite lysocline. Journal of Gephysical Research 96, 17037-17050. 
Arrhenius, G. 1952. Properties of the sediment and their distribution: Sediment cores from the East Pacific. Swedish Deep-Sea Expedition 1947-1948, 5, 1-228.

Baumann, K.-H., Sprengel, C. 2000. Morphological variations of selected coccolith species in a sediment trap north of the Canary Islands. Journal of Nannoplankton Research 22, 185-193.

Baumann, K.-H., Freitag, T. 2004. Pleistocene fluctuations in the northern Benguela Current system as revealed by coccolith assemblages. Marine Micropaleontology 52, 195-215.

Benway, H., Mix, A. 2004. Oxygen isotopes, upper-ocean salinity, and precipitation sources in the eastern tropical Pacific. Earth and Planetary Science Letters 224, 493-507.

Baumann, K.-H., Andruleit, H., Samtleben, C. 2000. Coccolithophores in the Nordic Seas: comparison of living communities with surface sediment assemblages. Deep-Sea Research II 47, 1743-1772.

Baumann, K.-H., Böckel, B., Frenz, M. 2004. Coccolith contribution to South Atlantic carbonate sedimentation. In: Thierstein, H.R., Young, J.R. (Eds.), Coccolithophores - from molecular processes to global impact. Springer-Verlag, Berlin-Heidelberg, pp. 367-402.

Baumann, K.-H., Andruleit, H., Böeckel, B., Geisen, M., Kinkel, H. 2005. The significance of extant coccolithophores as indicators of ocean water masses, surface water temperature, and paleoproductivity: a review. Paläontologishce Zeitschrift 79, 93-112.

Beaufort, L., Lancelot, Y. Camberlin, P., Cayre, O., Vincent, E., Bassinot, F., Labeyrie, L. 1997. Insolation cycles as a major control of Equatorial Indian Ocean primary production. Science 278, 14511454.

Blasco, D., Estrada, M., Jones, B. 1980. Relationship between the phytoplankton distribution and composition and the hydrography in the North-West African upwelling region near Cabo Corbeiro. Deep-Sea Research 27, 799-819.

Böeckel, B., Baumann, K.-H. 2004. Distribution of coccoliths in surface sediments of the south-eastern South Atlantic Ocean: ecology, preservation and carbonate contribution. Marine Micropaleontology 51, 301-320.

Bollmann, J. 1997. Morphology and biogeography of Gephyrocapsa coccoliths in Holocene sediments. Marine Micropaleontology 29, 319-350.

Bollmann, L., Baumann, K.-H., Thierstein, H.R. 1998. Global dominance of Gephyrocapsa coccoliths in the Late Pleistocene: Selective dissolution, evolution or global environment change. Paleoceanography $13,517-529$.

Brand, L.E. 1994. Physiological ecology of marine coccolithophores. In: Winter, A., Siesser, W.G. (Eds.). Coccolithophores, Cambridge University Press, Cambridge, pp. 39-62.

Broerse, A.T.C., Brummer, G.J.A., van Hinte, J.E. 2000. Coccolithophore export production in response to monsoonal upwelling off Somalia (northwestern Indian Ocean). Deep-Sea Research Part 2, Topical Studies in Oceanography 47, 2179-2205.

Cachão, M., Moita, M. T. 2000. Coccolithus pelagicus, a productivity proxy related to moderate fronts off Western Iberia. Marine Micropaleontology 39, 131-155.

Castradori, D. 1993. Calcareous nannofossils and the origin of eastern Mediterranean sapropel. Paleoceanography 8, 459-471.

Colmenero-Hidalgo, E., Flores, J.A., Sierro, J., Barcena, M.A., Lowemark, L., Schonfeld, J., Grimalt, J.O., 2004. Ocean surface water response to short-term climate changes revealed by coccolithophores from the Gulf of Cadiz (NE Atlantic) and Alboran Sea (W Mediterranean). Paleogeography, Paleoclimatology, Paleoecology 205, 317-336.

Cortes, M.Y., Bollmann, J., Thierstein, H. 2001. Coccolithophore ecology at the HOT station ALOHA Hawaii. Deep-Sea Research II 48, 1957-1981.

Di Stefano, E., Incarbona, A. 2004. High-resolution paleoenvironmental recostruction of ODP Hole 963 D (Sicily Channel) during the last deglaciation based on calcareous nannofossils. Marine Micropaleontology 52, 241-254.

Ericson, D.B., Wollin, G. 1956. Correlation of six cores from the equatorial Atlantic and the Caribbean. Deep-Sea Research 3, 104-125.

Estrada, M., 1978. Mesoscale heterogeneities of the phytoplankton distribution. In: Boje, R., Tomezak, M. (Eds.), The Upwelling Region of northWest Africa. (Upwelling Ecosystems). Springer, Berlin, pp. 15-23.

Farrell, J.W., Prell, W. 1989. Climatic change and $\mathrm{CaCO}_{3}$ preservation: $\mathrm{An} 800,000$ year bathymetric reconstruction from the Central Equatorial Pacific Ocean. Paleoceanography 4, 447-466. 
Farrell, J.W., Prell, W. 1991. Pacific $\mathrm{CaCO}_{3}$ Preservation and $\delta^{180}$ Since 4 Ma: Paleoceanic and Paleoclimatic Implications. Paleoceanography 6, 485-498.

Fiedler, P.C. 2002. The annual cycle and biological effects of the Costa Rica Dome. Deep-Sea Research I 49, 321-338.

Fincham, M.J., Winter, A., 1989. Paleoceanographic interpretations of coccoliths and oxygen-isotopes from sediments from the surface of the Southwest Indian Ocean. Marine Micropaleontology 13, 325-351.

Findlay, C.S., Giraudeau, J. 2002. Movement of oceanic fronts south Australia during the last $10 \mathrm{ka}$ interpretation of calcareous nannoplankton in surface sediments from the Southern Ocean. Marine Micropaleontology 46, 431-444.

Flores, J.A., Sierro, F.J., Raffi, I., 1995. Evolution of the calcareous nannofossil assemblage as a response to the paleoceanographic change in the Eastern Equatorial Pacific from 4 to $2 \mathrm{Ma}$ (leg 138, Site 849 and 852). Proc. ODP, Initial Rep. 138, 163-176.

Flores, J.A., Sierro, F.J., Frances, G., Vazquez, A., Zamarreno, I., 1997. The last 1000.000 years in the western Mediterranean: sea surface water and frontal dynamics as revealed by coccolithophores. Marine Micropaleontology 29, 351-366.

Flores, J.A., Gersonde, R., Sierro, F.J. 1999. Pleistocene fluctuations in the Agulhas current retroflection based on the calcareous plankton record. Marine Micropaleontology 37, 1-22.

Flores, J.A., Bárcena, M.A., Sierro, F.J., 2000. Ocean-surface and wind dynamics in the Atlantic Ocean off Northwest Africa during the last 140000 years. Palaeogeography, Palaeoclimatology, Palaeoecology 161, 459-478.

Flores, J.A., Marino, M., Sierro, F.J., Hodell, D.A. y Charles, C.D., 2003. Calcareous plankton dissolution pattern and coccolithophore assemblages during the last $600 \mathrm{kyr}$ at ODP Site 1089 (Cape Basin, South Atlantic): paleoceanographic implications. Palaeogeography, Palaeoclimatology, Palaeoecology 196, 409-426.

Flores, J.-A., Sierro, F.J., Filippelli, G.M., Bárcena, M.Á., Pérez-Folgado, M., Vázquez, A., Utrilla, R., 2005. Surface water dynamics and phytoplankton communities during deposition of cyclic late Messinian sapropel sequences in the western Mediterranean. Marine Micropaleontology 56, 5079.

Flores, J.-A., Wei, W., López-Otálvaro, G.E., Alvarez, C., Sierro, F.J. 2006. In: Tiedemann, R., Mix, A.C., Richter, C., Ruddiman, W.F. (Eds.), Data Report: Tropical and Equatorial Calcareous Nannofossil Pleistocene Biostratigraphy, ODP Leg 202. Proc. ODP, Sci. Results, vol. 202. College Station, TX, Ocean Drilling Program, pp. 1-10. doi:10.2973/odp.proc.sr.202.214.2006.

Gard, G., Backman, J., 1990. Synthesis of Arctic and Sub-Arctic coccolith biochronology and History of North Atlantic drift water influx during the last 500,000 years. In: Bleil, U., Thiede, J. (Eds.), Geological History of the Polar Oceans: Arctic Versus Antarctic. Kluwer, pp. 417-436.

Gartner, S. 1988. Paleoceanography of the mid-Pleistocene. Marine Micropaleontology 12, 23-46.

Gartner, S., Chow, J., Stanton, R.J. 1987. Late Neogene paleoceanography of the eastern Caribbean, the Gulf of Mexico, and the eastern Equatorial Pacific. Marine Micropaleontology 12, 255-304.

Geitzenauer, K.R., Roche, M.B., Mclntyre, A., 1976. Modern Pacific coccoliths assemblages: derivation and application to Late Pleistocene paleotemperature analysis. In: Cline, R.M., Hays, J.D. (Eds.), Investigation of Late Quaternary Paleoceanography and Paleoclimatology. Geological Society of America, vol. 145, pp. 423-448

Giraudeau, J. 1992. Distribution of Recent nannofossils beneath the Benguela system: Southwest African continental margin. Marine Geology 108, 219-237.

Giraudeau, J., Baley, G.W. 1995. Spatial dynamics of coccolithophore communities during an upwelling event in the Southern Benguela system. Continental Shelf Research 15, 1825-1852.

Giunta, S., Negri, A., Morigi, C., Capotondi, L., Combourieu-Nebout, N., Emeis, K.C., Sangiorgi, F., Vigliotti, L. 2003. Coccolithophorid ecostratigraphy and multi-proxy paleoceanographic reconstruction in the Southern Adriatic Sea during the last deglacial time (Core AD91-17). Paleogeography, Paleoclimatology, Paleoecology 190, 39-59.

Hagino, K., Okada, H., Matsuoka, H. 2000. Spatial dynamics of coccolithophore assemblages in the Equatorial western-central Pacific Ocean. Marine Micropaleontology 39, 53-72.

Haidar, A.T., Thierstein, H.R. 1997. Calcareous phytoplankton dynamics at Bermuda (North Atlantic). European Geosciences Union (EGU) 9 Abstract Supplement 1. Terra Nova 9, p. 602. 
Hastenrath, S., Druyan, L. 1993. Circulation anomaly mechanisms in the tropical Atlantic sector during the Northeast Brazil rainy season: results from the GISS General Circulation Model. Journal of Geophysical Research-Atmospheres 98 (D8), 14017-14923.

Hastenrath, S., Greischar, L. 1993. Circulation mechanisms related to northeast Brazil rainfall anomalies. Journal of Geophysical Research 98 (D3), 5093-5102.

Henriksson, A.S. 2000. Coccolithophore response to oceanographic changes in the equatorial Atlantic during the last 200,000 years. Paleogeography, Paleoclimatology, Paleoecology 156, 161-173.

Hiramatsu, C., De Deckker, P. 1997. The calcareous nannoplankton assemblages of surface sediments in the Tasman and Coral Seas. Palaeogeography, Palaeoclimatology, Palaeoecology 131, 257-285.

Honjo, S., Okada, H. 1974. Community structure of coccolithophores in the photic layer of the mid-Pacific. Micropaleontology 20, 209-230

Houghton, S.D. and Guptha, M.V.S. 1991. Monsoonal and fertility controls on Recent marginal sea and continental fertility controls on Recent marginal sea and continental northern Indian oceans, Marine Geology 97, 251-259.

Jordan, R.W., Chamberlain, A.H.L. 1997. Biodiversity among Haptophyte algae. Biodiversity and Conservation 6, 131-152.

Jordan, R.W., Winter, A. 2000. Assemblages of coccolithophorids and other living microplankton off the coast of Puerto Rico during January-May 1995. Marine Micropaleontology 39, 113-130.

Jordan, R.W., Zhao, M., Eglinnton, G., Weaver, P.P.E. 1996. Coccolith and alkenone stratigraphy and paleoceanography at an upwelling site off NW Africa (ODP 658C) during the last 130,000 years. In: Moguilevsky, A., Whatley, R. (Eds.), Microfossils and Oceanic Environments. Aberystwyth Press, University of Wales, pp. 111-130.

Karlin, R., Lyle, M., Zahn, R. 1992. Carbonate variations in the Northeast Pacific during the late Quaternary. Paleoceanography 7, 43-61.

Kennett, J.P., Huddlestun, P. 1972. Late Pleistocene Paleoclimatology, foraminiferal biostratigraphy and tephrochronology, western Gulf of Mexico. Quaternary Research 2, 38-69.

Khodri, M., Ramstein, G., de Noblet-Ducoudré, N. 2003. Sensitivity of the northern extratropics hydrological cycle to the changing insolation forcing at 126 and $115 \mathrm{ky}$ BP. Climate Dynamics 21, 273-287. doi: 10.1007/s00382-003-0333-5.

Kinkel, H., Baumann, K.-H., Cepek, M. 2000. Coccolithophores in the equatorial Atlantic Ocean: response to seasonal and Late Quaternary surface water variability, Marine Micropaleontology 39, 87-112.

Klejine, A., 1993. Morphology, Taxonomy and Distribution of extant Coccolithophorids (calcareous nannoplankton). Ph. D. Thesis, Vrije Universiteit, Amsterdam, $321 \mathrm{p}$.

Kleijne, A., Kroon, D., Zevemboom,W.,1989. Phytoplankton and foraminiferal frequencies in northern Indian Ocean and Red Sea surface waters. Netherlands Journal of Sea Research 24, 531-539.

Knappertsbusch, M. 1993. Geographic distribution of living and Holocene coccolithophores in the Mediterranean Sea. Marine Micropaleontology 21, 219-247.

Knappertsbusch, M., Cortes, M.Y., Thierstein, H.R. 1997. Morphologic variability of the coccolithophorid Calcidiscus leptoporus in the plankton, surface sediments and from the Early Pleistocene. Marine Micropaleontology 30, 293-317.

Lea, D.W., Pak, D.K., Belanger, C.L., Spero, H.J., Hall, M.A., Shackleton, N.J. 2006. Paleoclimate history of Galápagos surface waters over the last 135,000 yr. Quaternary Science Reviews 25, 11521157.

Liu, C., Wang, P., Tian, J., Cheng, X. 2008. Coccolith evidence for Quaternary nutricline variations in the southern South China Sea. Marine Micropaleontology, doi:10.1016/j.marmicro.2007.11.008.

Lohmann, H. 1920. Die Coccolithophoridae, eine Monographie der Coccolithen bildenden Flagellaten, zugleich ein Beitrag zur Kenntnis des Mittelmeerauftriebs, Arch. Protistenkd. 1, pp. 89-165.

Lyle, M., Mix, A., Pisias, N. 2002. Patterns of $\mathrm{CaCO} 3$ deposition in the eastern tropical Pacific Ocean for the last $150 \mathrm{kyr}$ : Evidence for a southeast Pacific depositional spike during marine isotope stage (MIS)2. Paleoceanography 17, doi: 10.1029/2000PA000538.

Lisiecki, L.E., Raymo, M.E. 2005. A Pliocene-Pleistocene stack of 57 globally distributed benthic $\delta^{18} \mathrm{O}$ records. Paleoceanography 20, doi:10.1029/2004PA001071.

Maiorano, P., Marino, M. 2004. Calcareous nannofossil bioevents and environmental control on temporal and spatial patterns at the early middle Pleistocene. Marine Micropaleontology 53, 405-422. 
McIntyre, A., Bè, A.H.W. 1967. Modern coccolithophores of the Atlantic Ocean-I. Placolith and cyrtoliths. Deep-Sea Research 14, 561-597.

McIntyre, A., Bè, A.H.W., Roche, M.B. 1970. Modern Pacific Coccolithophorida: a paleontological thermometer. Transactions of the New York Academy of Science Series II 32, 720-731.

Marino, M., Maiorano, P., Lirer, F. 2008. Changes in calcareous nannofossil assemblages during the midPleistocene Revolution. Marine Micropaleontology, doi:10.1016/j.marmicro.2007.11.010.

Martínez, J. I., Mora, G., Barrows, T.T. 2007. Paleoceanographic conditions in the western Caribbean Sea for the last $560 \mathrm{Ka}$ as inferred from planktonic foraminifera. Marine Micropaleontology 64, 177188.

Masson, S. Delecluse, P. 2000. Influence of the Amazon River runoff on the tropical Atlantic. Phys. Chem. Earth 26, 137-142.

Mitchell-Innes, B. A., Winter, A., 1987. Coccolithophores: a major phytoplankton component in mature upwelled waters off Cape Peninsula, South Africa in March, 1983. Marine Biology 95, 25-30.

Mix, A.C., Tiedemann, R., Blum, P., et al., 2003. Proc. ODP, Init. Repts., 202. Ocean Drilling Program, College Station, TX. doi:10.2973/odp.proc.ir.202.2003.

Molfino, B. R., Mclntyre, A. 1990. Nutricline variation in the equatorial Atlantic coincident with the Younger Dryas. Paleoceanography 5, 997-1008.

Molfino, B., McIntyre, A. 1990. Precessional forcing of nutricline dynamics in the equatorial Atlantic. Science 249, 766-769.

Müller, C. 1985. Late Miocene to recent Mediterranean biostratigraphy and paleoenvironments based on calcareous Nannoplankton. In: Stanley, D.J., Wezel, F.C. (Eds.), Geological Evolution of the Mediterranean Basins. Springer, New York, pp. 471-485.

Narciso, A., Cachão, M., de Abreu, L., 2006. Coccolithus pelagicus subsp. pelagicus versus Coccolithus pelagicus subsp. braarudii (Coccolithophore, Haptophyta): A proxy for surface subartic Atlantic waters off Iberia during the last 200 kyr. Marine Micropaleontology 59, 15-34.

Negri, A., Giunta, S. 2001. Calcareous nannofossil paleoecology in the sapropel S1 of the eastern lonian Sea: paleoceanographic implications. Paleogeography, Paleoclimatology, Paleoecology 169, 101-112.

Negri, A., Capotondi, L., Keller, J. 1999. Calcareous nannofossils, planktonic foraminifera and oxygen isotopes in the late Quaternary sapropels of the Ionian Sea. Marine Geology 157, 89-103.

Negri, A., Morigi, G., Giunta, S., 2003. Are productivity and stratification important to sapropel deposition? Microfossil evidence from late Pliocene insolation cycle 180 at Vrica, Calabria. Paleogeography, Palaeoclimatology, Palaecology 190, 243-255.

Okada, H., Honjo, S., 1973. The distribution of oceanic coccolithophorids in the Pacific. Deep-Sea Research 20, 355-374.

Okada, H., Mclntyre, A., 1977. Modern coccolithophores of the Pacific and North Atlantic Oceans. Micropaleontology 23, 1-55.

Okada, H., McIntyre, A., 1979. Seasonal distributions of Modern coccolithophores in the western Atlantic ocean. Marine Biology 54: 319-328.

Okada, H., Wells, P., 1997. Late Quaternary nannofossil indicators of climate change in two deep-sea cores associated with the Leeuwin Current off Western Australia. Palaeogeography, Palaeoclimatology, Palaeoecology 131, 413-432.

Pailler, K., Bourlès, B., Gouriou, Y. 1999. The Barrier layer in the Western tropical Atlantic Ocean. Geophysical Research Letters 26, 2069-2072.

Parente, A., Cachão, M., Baumann, K.-H., de Abreu, L., Ferreira, J. 2004. Morphometry of Coccolithus pelagicus s.l. (Coccolithophore, Haptophyta) from offshore Portugal, during the last $200 \mathrm{kyr}$. Micropaleontology 50 (supplement), 107-120.

Pedersen, T.F. 1983. Increased productivity in the eastern equatorial Pacific during the last glacial maximum (19,000 to 14,000 yr B.P.). Geology 11, 16-19.

Peterson, L.C., Haug, G.H., Hughen, K.A., Röhl, U. 2000. Rapid changes in the hydrologic cycle of the Tropical Atlantic during the Last Glacial. Science 290, 1947-1951, 2000.

Pujos-Lamy, A. 1977. Emiliania et Gephyrocapsa (Nannoplancton calcaire): Biométrie et intéret biostratigraphique dans le Pleistocène supérieur marin des Açores. Revista Española de Micropaleontología 9, 69-84. 
Pujos, M., Froidefond, J.-M. 1995. Water masses and suspended matter circulation on the French Guiana continental shelf. Continental Shelf Research 15, 1157-1171.

Quinn, P.S., Saez, G.A., Baumann, K.-H., Steel, B.A., Sprengel, C., Medlin, L. 2004. Coccolithophorid biodiversity: evidence from the cosmopolitan species Calcidiscus leptoporus. In: Thierstein, H.R., Young, Y.R. (Eds.), Coccolithophores from Molecular Processes to Global Impact. SpringerVerlag, Berlin, pp. 299-326.

Raffi, I., 2002. Revision of the early-middle Pleistocene calcareous nannofossil biochronology (1.75-0.85 Ma). Marine Micropaleontology 45, 25-55.

Raffi, I., Backman, J., Rio, D., Shackleton, N.J. 1993. Early Pleistocene and late Pliocene nannofossil biostratigraphy and calibration to oxygen isotope stratigraphies from DSDP Site 607 and ODP Site 677. Paleoceanography 8, 387-404.

Roth, P.H. 1994. Distribution of coccoliths in oceanic sediments. In: Winter, A., Siesser, W.G. (Eds.), Coccolithophores, Cambridge University Press, Cambridge, pp. 199-218.

Roth, P.H., Berger, W.H. 1975. Distribution and dissolution of coccoliths in the South and Central Pacific. Dissolution of Deep-sea Carbonates. Cushman Foundation for Foraminiferal Research, Special Publication 13, 87-113.

Roth, P.H., Coulbourn,W.T. 1982. Floral and solution patterns of coccoliths in surface sediments of the North Pacific. Marine Micropaleontology 7, 1-52.

Servain, J., Wainer, J., McCreary, Jr., Dessier, A. 1999. Relationship between the equatorial and meridional modes of climatic variability in the tropical Atlantic. Geophysical Research Letters 26, 485-488.

Shackleton, N.J., Berger, A., Peltier, W.R., 1990. An alternative astronomical calibration of the Pleistocene timescale based on ODP Site 677. Transactions of the Royal Society of Edinburgh: Earth Sciences 81, 251-261.

Showers, W.J., Bevis, M. 1988. Amazon cone isotopic stratigraphy: evidence for the source of the tropical freshwater spike. Palaeoecology, Palaeogeography, Palaeoclimatology 64, 189-199.

Sprovieri, R., Di Stefano, E., Incarbona, A., Gargano, M.E. 2003. A High resolution record of the last deglaciation in the Sicily channel based on foraminifera and calcareous nannofossil quantitative distribution. Paleogeography, Paleoclimatology, Paleoecology 202, 119-142.

Takahashi, K., Okada, H. 2000. The paleoceanography for the last 30, 000 years in the southeastern Indian Ocean by means of calcareous nannofossils. Marine Micropaleontology 40, 83-103.

Tanaka, Y., 1991. Calcareous nannoplankton thanatoconosis in surface sediments from seas around Japan. Sci. Rep. Tohoku University, 2nd Ser. (Geol.) 61, 127-198.

Thierstein, H.R., Geitzenauer, K.R., Molfino, B., Shackleton, N.J., 1977. Global synchroneity of late Quaternary coccolith datum levels: validation by oxygen isotopes. Geology 5, 400-404.

Vink, A., Rühlemann, C., Zonneveld, K., Mulitza, S., Hüls, M., Willems, H. 2001. Shifts in the position of North Equatorial current and rapid productivity changes in the western Tropical Atlantic during the last glacial. Paleoceanography 16, 1-12.

Weaver, P.P.E., Pujol, C., 1988. History of the last deglaciation in the Alboran Sea (western Mediterranean) and adjacent North Atlantic as revealed by coccolith floras. Paleogeography, Paleoclimatology, Paleoecology 64, 35-42.

Wei, W. 1993. Calibration of upper Pliocene-lower Pleistocene nannofossil events with oxygen isotope stratigraphy. Paleoceanography 8, 85-89

Winter, A. 1982. Paleoenvironmental interpretation of Quaternary coccolith assemblages from the Gulf of Aquaba (Elat), Red Sea. Revista Española de Micropaleontología 14, 291-314.

Winter, A., Jordan, R.W., Roth, P.H. 1994. Biogeography of living Coccolithophores in ocean waters. In: Winter, A., Siesser, W.G. (Eds.), Coccolithophores. Cambridge University Press, Cambridge, pp. 161-178.

Xie, S.-P., Tanimoto, Y., Noguchi, H., Matsuno, T. 1999. How and why climate variability differs between the tropical Atlantic and Pacific. Gephysical Research Letters 26, 1609-1612.

Young, J.R., 1994. Functions of coccoliths. In: Winter, A. Siesser, W.G. (Eds.), Coccolithophores. Cambridge University Press, Cambridge, pp. 63-82.

Ziveri, P., Thunell, R.C. 2000. Coccolithophores export production in Guaymas Basin, Gulf of California: response to climatic forcing. Deep-Sea Research II 47, 2073-2100. 
Ziveri, P., Thunell, R.C., Rio, D. 1995. Export production of coccolithophores in an upwelling region: results from San Pedro Basin, Southern California Borderlands. Marine Micropaleontology 24, 335-358.

Ziveri, P., Baumann, K.-H., Boeckel, B., Bollmann, J., Young, J. 2004. Biogeography of selected Holocene coccoliths in the Atlantic Ocean. In: Thierstein, H.R., Young, Y.R. (Eds.), Coccolithophores from Molecular Processes to Global Impact. Springer-Verlag, Berlin, pp. 403-428. 
Conclusiones 
1. Los resultados obtenidos en esta tesis doctoral sugieren que el registro de la asociación de cocolitóforos muestra una variabilidad temporal e interlatitudinal considerable, caracterizando la respuesta de los sectores tropicales de los océanos Pacífico oriental y Atlántico occidental a los cambios en las condiciones paleoceanográficas y paleoatmosféricas durante los últimos ciclos climáticos.

2. El análisis de la preservación pone de manifiesto que en términos generales ésta varía entre buena a moderada en todos los testigos. Algunos intervalos relativamente cortos en los testigos 1242 y MD03-2616 evidencian una preservación pobre, llegando a la desaparición completa de cocolitos en algunos casos, enmascarando la señal micropaleontológica de los registros. Sin embargo, la preservación buena a moderada predomina en el resto de las secuencias, sugiriendo que los ejemplares estudiados son fiel reflejo de la asociación de los cocolitóforos dominante en cada momento, lo que es especialmente significativo en los testigos ODP 1242 y MD03-2616.

3. La combinación de la estratigrafía isotópica, los datos paleomagnéticos, la definición de una serie de biohorizontes de cocolitóforos en el Pacífico oriental tropical, la Biozonación Climática de foraminíferos planctónicos en el Atlántico occidental tropical y la estratigrafía del carbonato de los cocolitos permitieron la elaboración de un marco cronoestratigráfico extraordinario para los testigos estudiados, el cual es correlacionable en sus respectivas regiones tropicales.

4. La variabilidad del carbonato aportado por los cocolitos siguió el patrón del índice $\mathrm{N}$ de paleoproductividad, indicando que el perfil de carbonato de los cocolitos puede ser utilizado como un indicador adicional de paleoproductividad.

5. Los perfiles de paleoproductividad revelaron que la zona de divergencia ecuatorial está caracterizada como una zona de altísima paleoproductividad en correspondencia con una máxima somerización de la nutri-termoclina. La variabilidad de la paleoproductividad de los cocolitóforos en el Pacífico oriental ecuatorial no siguió un patrón glacial/interglacial, indicando que la dinámica de los vientos alisios permitió la llegada continua de aguas enriquecidas en nutrientes a través de la célula de surgencia, observándose algún cambio drástico influido por otros eventos no directamente vinculados a los ciclos glacial/interglacial, por ejemplo, los eventos "El Niño". 
6. De otro lado, el registro micropaleontológico en la "warm pool" del Pacífico oriental tropical, sugirió que aunque la paleoproductividad es alta, es menor que la registrada en la zona de divergencia ecuatorial. La paleoproductividad en el sector de la "warm pool" siguió el patrón glacial/interglacial, indicando máximos durante los glaciales. El incremento en la paleoproductividad durante los glaciales en el Pacífico oriental tropical estuvo favorecido por la somerización de la nutri-termoclina debida a la intensificación de los vientos alisios del noreste. Esta dinámica de los vientos alisios del noreste activó las células de surgencia costera producidas por los "wind jets" de Panamá, Papagayo y Tehuantepec que impidieron además, la llegada masiva de aguas cálidas desplazadas por la contracorriente Norecuatorial (NECC) hacia el Pacífico oriental. Por el contrario, la intensificación de los vientos alisios del sureste durante los interglaciales, favorecieron la llegada del régimen cálido de la NECC, profundizando la nutritermoclina en el Pacífico oriental tropical. La intensificación de los vientos alisios del sur, también favoreció el desarrollo del Domo de Costa Rica, impidiendo que la nutri-termoclina en el Pacífico oriental tropical se profundizara aún más por la llegada del régimen cálido de la NECC. De esta manera se puede decir que aunque la paleoproductividad y la nutri-termoclina fluctuaron a escala glacial/interglacial, el océano Pacífico oriental tropical estuvo caracterizado por un régimen relativamente eutrófico. Este patrón de paleoproductividad es correlacionable con el patrón glacial/interglacial de producción de carbonato en los océanos Pacífico e Índico previamente sugerido por otros autores. Adicionalmente se pudo observar que la paleoproductividad fue disminuyendo hacia el norte del Pacífico oriental en la cuenca de Panamá, lo cual concuerda con la topografía actual de la nutri-termoclina.

7. El evento "mid-Brunhes" fue caracterizado rigurosamente por la ocurrencia del taxón G. caribbeanica, un componente extra, mayoritario y dominante en términos de abundancia y masa de carbonato aportado durante el intervalo comprendido entre los MIS 15 a 8 (615 Ka a $283 \mathrm{Ka}$ ) en la cuenca de Panamá.

8. Un análisis comparativo de la variación de la paleoproductividad en la cuenca de Panamá permitió identificar tres intervalos análogos a los eventos de la El Niño - la Oscilación del Sur": El Intervalo 1 (MIS 22 -925 Ka al 6 -128 Ka) registró la máxima paleoproductividad producida por una fuerte somerización de la nutri-termoclina, la intensificación de los vientos alisios y la posición de la ITCZ siempre en el Hemisferio Norte. Este intervalo fue asociado con un evento análogo a "La Niña" con algunas fluctuaciones. El Intervalo 2 (MIS 5; $128 \mathrm{Ka}$ a $71 \mathrm{Ka}$ ) está representado por una drástica reducción en la productividad producida por la profundización de 
la nutri-termoclina, indicando el colapso de la surgencia de aguas en la zona de divergencia ecuatorial y en la "warm pool" debido al debilitamiento de los vientos alisios y el desplazamiento de la ITCZ hacia el ecuador; lo cual pudo ser comparado con un evento análogo a "El Niño" con algunas oscilaciones. El Intervalo 3 (MIS 4 a 1; últimos $71 \mathrm{Ka}$ ) está representado por el incremento en la productividad superficial y la somerización de la nutri-termoclina en la zona de divergencia ecuatorial, mostrando una clara analogía con las condiciones observadas durante un evento "La Niña" con ciertas oscilaciones, pero más moderado que el registrado en el Intervalo 1. Los testigos 1241 y 1242 no pudieron recuperar la estructura del océano superficial previa al MIS 5 , indicando que la intensificación de los vientos alisios no fue tan extrema como para favorecer la productividad en la "warm pool" del Pacífico oriental tropical.

9. Los cambios en el registro de paleoproductividad en la cuenca de la Guayana fueron mayores durante el MIS 5 que durante los estadios relativamente más fríos 4 al 2 y al comienzo del Holoceno (71 Ka a $5 \mathrm{Ka}$ ). El patrón de paleoproductividad además, mostró máximos durante los subestadios interglaciales de los MIS 5 y 3 . Esta variabilidad indica que durante los interglaciales, los vientos alisios del sureste se intensificaron, desplazaron la ITCZ hacia el norte y soplaron paralelos a la costa, favoreciendo la activación de la surgencia costera y la somerización de la nutri-termoclina debido a la retirada de aguas cálidas a través de la NECC. El dominio de los alisios del noreste durante los eventos fríos indica la reducción de la paleoproductividad y la profundización de la nutri-termoclina debido al debilitamiento de la célula de surgencia costera y de la NECC; mientras provocaban el desplazamiento de la ITCZ hacia el sur. Esta dinámica paleoceanográfica siguió el patrón de insolación de verano a los $65^{\circ} \mathrm{N}$ (indicando máximos de paleoproductividad durante máximos de insolación), y sugiere una conexión interlatitudinal entre la insolación de verano en el Hemisferio Norte, la ITCZ y la dinámica de los vientos alisios del sureste durante el último ciclo climático. 
$\Delta$ péndices 


\section{Apéndice A: Lista de los taxones estudiados en esta Tesis Doctoral}

Calcidiscus leptoporus (Murray and Blackman, 1898) Loeblich and Tappan, 1978

Calciosolenia murrayi Gran, 1912

Coccolithus pelagicus (Wallich, 1877) Schiller, 1930

Emiliania huxleyi (Lohmann, 1902) Hay and Mohler in Hay et al., 1967

Florisphaera profunda Okada and Honjo, 1973

Gephyrocapsa aperta Kamptner, 1963

Gephyrocapsa ericsonii McIntyre and Bé, 1967

Gephyrocapsa caribbeanica Boudreaux and Hay, 1967

Gephyrocapsa muellerae Bréhéret, 1978

Gephyrocapsa oceanica Kamptner, 1943

Hayaster perplexus (Bramlette and Riedel 1954) Bukry 1973

Helicosphaera carteri (Wallich, 1877) Kamptner, 1954

Neosphaera coccolithomorpha Lecal-Schlauder, 1950

Oolithotus (Cohen, 1964) Reinhardt, in Cohen and Reinhardt, 1968

Oolithotus antillarum Reinhardt, in Cohen and Reinhardt, 1968

Pontosphaera Lohmann, 1902

Pseudoemiliania lacunosa (Kamptner 1963) Gartner 1969

Rhabdosphaera clavigera (Murray and Blackman, 1898)

Reticulofenestra Hay, Mohler and Wade 1966

Syracosphaera Lohmann, 1902

Syracosphaera lamina Lecal-Schlauder 1951

Syracosphaera pulchra Lohmann, 1902

Umbellosphaera Paasche, in Markali and Paasche, 1955

Umbilicosphaera hulburtiana Gaarder, 1970

Umbilicosphaera sibogae var. foliosa (Kamptner, 1963) Okada and Mclntyre, 1977

Umbilicosphaera sibogae var. sibogae (Weber-van Bosse, 1901) Gaarder, 1970 
Apéndice B: Notas taxonómicas y diferencias morfológicas utilizadas en esta Tesis Doctoral para el género Gephyrocapsa (Adoptada de Fores et al., 2000)

\begin{tabular}{|c|c|c|c|c|}
\hline Esta Tesis Doctoral & $\begin{array}{l}\text { Small Gephyrocapsa (incluye G. } \\
\text { ericsonii y G.aperta) }\end{array}$ & $\begin{array}{l}\text { G. muellerae (incluye } G . \\
\text { margereli) }\end{array}$ & G. caribbeanica & $\begin{array}{l}\text { G. oceanica (incluye G. oceanica } \\
\text { y "large" Gephyrocapsa) }\end{array}$ \\
\hline Longitud del cocolitos & $\begin{array}{l}<3 \mu \mathrm{m} \text { (G. ericsonii); }<2.5 \mu \mathrm{m}(G . \\
\text { aperta) }\end{array}$ & $>3 \mu \mathrm{m}$ & $>3 \mu \mathrm{m}$ & $\begin{array}{l}\text { 3-4 } \mu \mathrm{m} \text { (G. oceanica) y } 4-5 \mu \mathrm{m} \\
\text { ("large" Gephyrocapsa) }\end{array}$ \\
\hline Ángulo del puente & & $5^{\circ}-40^{\circ}$ & Área central cerrada & $>50^{\circ}$ \\
\hline \multicolumn{5}{|l|}{ Autor } \\
\hline Thierstein et al., 1977 & & G. caribbeanica & & \\
\hline Raffi et al., 1993 & Small Gephyrocapsa & Small Gephyrocapsa & Small Gephyrocapsa & Medium Gephyrocapsa \\
\hline Bollmann, 1997 & G. minute & G. cold & G. oligotrophic, G. transitional & G. large, $G$. equatorial \\
\hline Flores et al., 2000 & Small Gephyrocapsa & G. muellerae & G. caribbeanica & G. oceanica \\
\hline Baumann y Freitag, 2004 & G. ericsonii / G. aperta & G. muellerae / G. margereli & G. caribbeanica & G.oceanica \\
\hline
\end{tabular}




\section{Apéndice C: Láminas fotográficas}
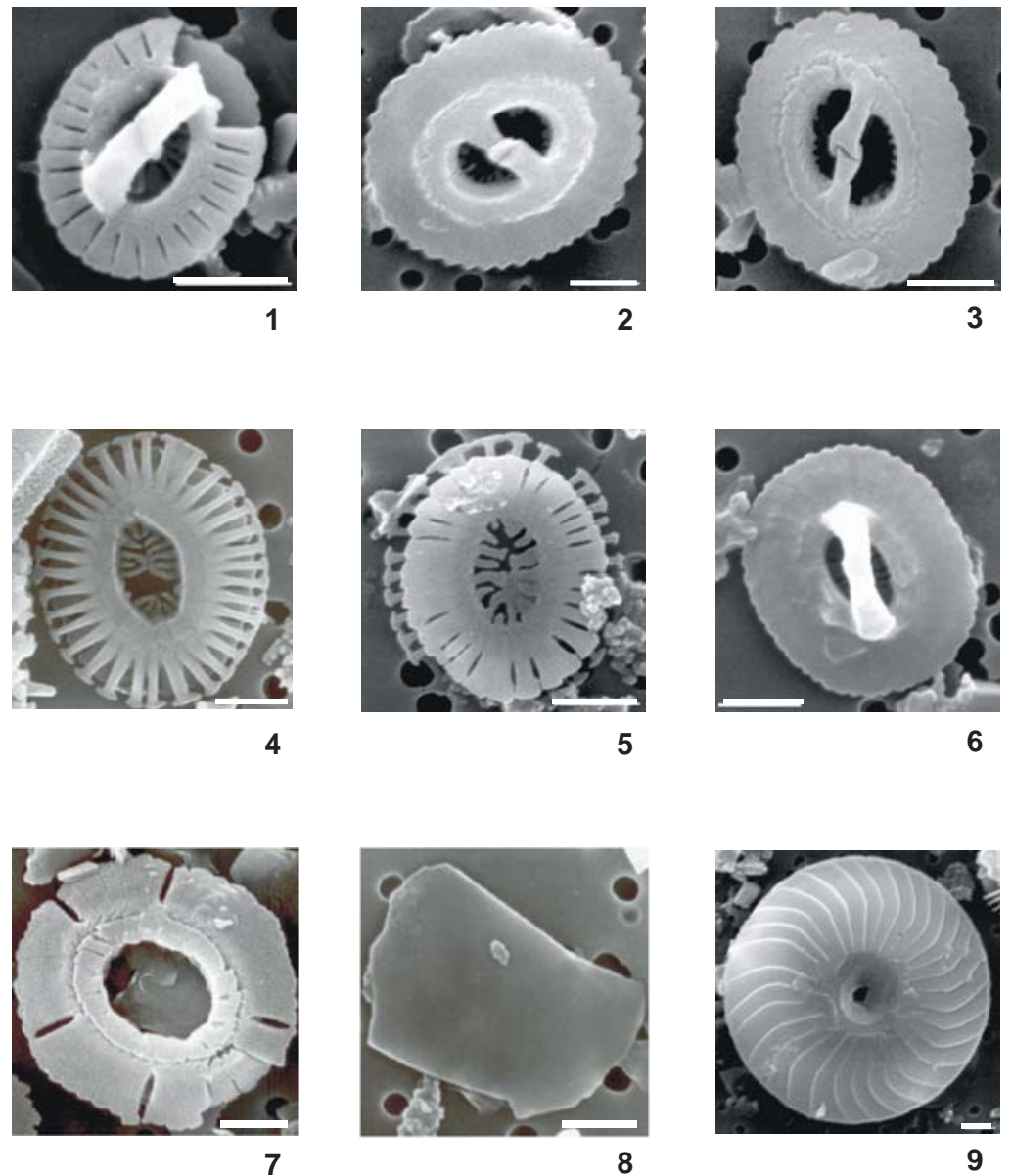

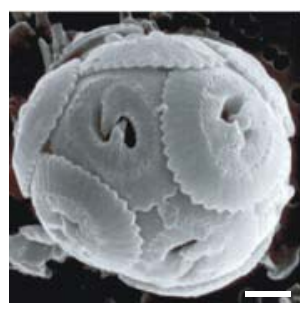

10

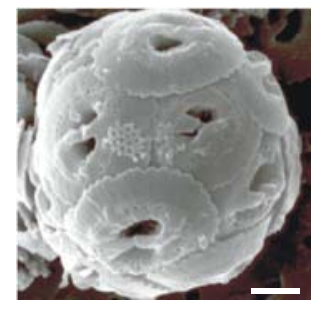

11

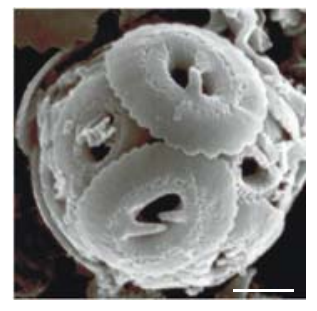

12

\section{Lámina I}

Las micrografías fueron tomadas con el microscopio electrónico de barrido ("Scanning Electron Microscope", SEM) en el Instituto de Geociencias de la Universidad de Kiel. (Escala de las barras: $1 \mu \mathrm{m}$ )

1. Gephyrocapsa ericsonii (ODP 1240; mcd 19.97). 2. Gephyrocapsa oceanica (ODP 1240; mcd 13.97). 3. Gephyrocapsa muellerae (ODP 1240; mcd 41.13). 4. Emiliania huxleyi (ODP 1240; mcd 1.53). 5. Emiliania huxleyi (ODP 1240; mcd 1.53). 6. Gephyrocapsa cf. caribbeanica (ODP 1240; mcd 12.48). 7. Pseudoemiliania lacunosa (ODP 1241; mcd 10.3). 8. Florisphaera profunda (ODP 1241; mcd 1.4). 9. Calcidiscus leptoporus (ODP 1240; mcd 5.34). 10. Gephyrocapsa caribbeanica - cocosfera (ODP 1240; mcd 41.68). 11. Gephyrocapsa oceanica - cocosfera (ODP 1240; mcd 41.68). 12. Gephyrocapsa oceanica - cocosfera (ODP 1240; mcd 41.68) 

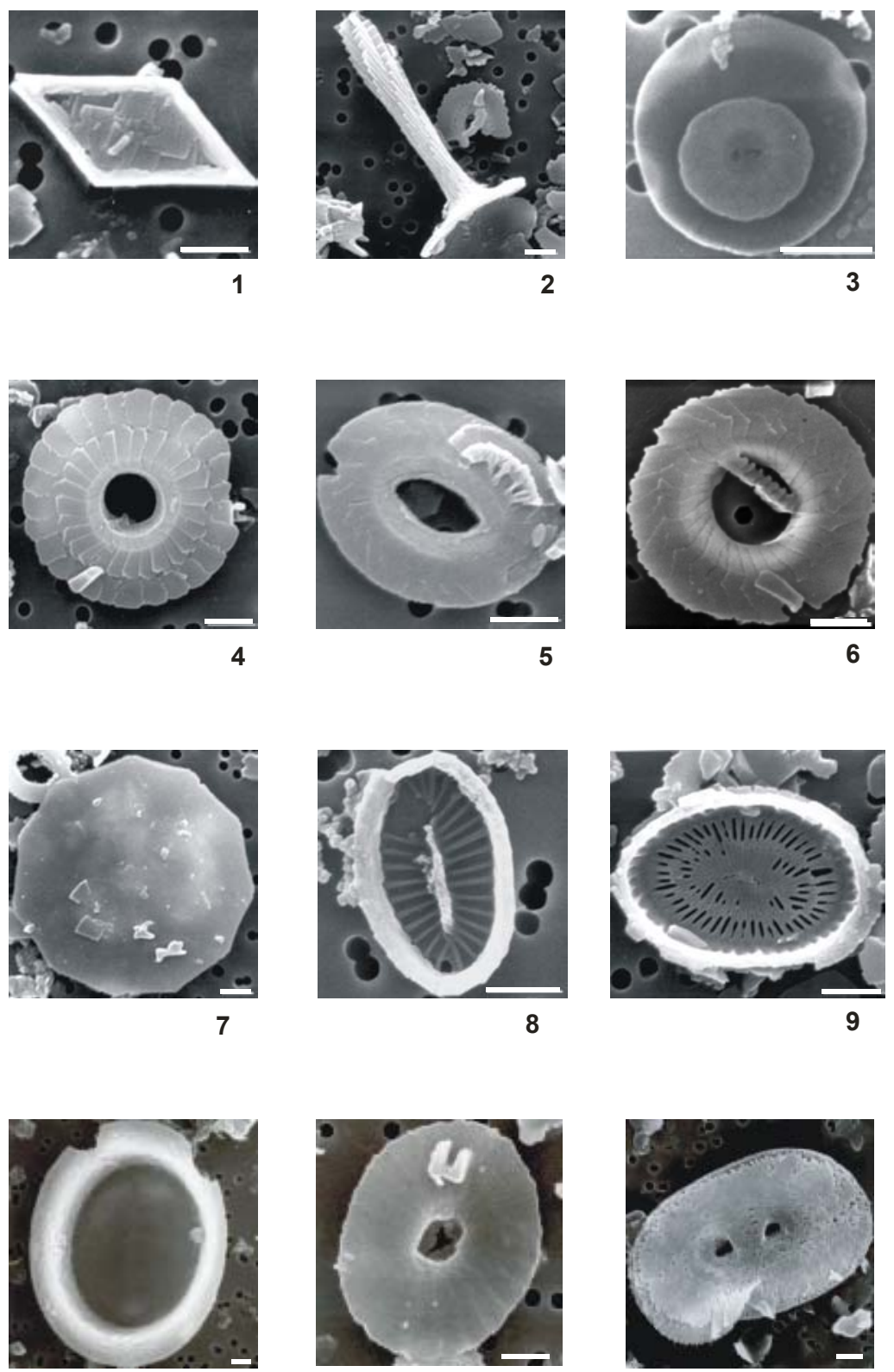

10

11

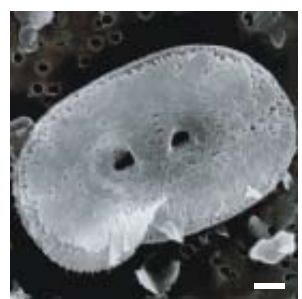

\section{Lámina II}

Las micrografías fueron tomadas con el microscopio electrónico de barrido ("Scanning Electron Microscope", SEM) en el Instituto de Geociencias de la Universidad de Kiel. (Escala de las barras: $1 \mu \mathrm{m}$ )

1. Calciosolenia murrayi (ODP 1240; mcd 19.97). 2. Rhabdosphaera clavigera (ODP 1240; mcd 41.68). 3. Oolithotus antillarum (ODP 1240; mcd 1.53). 4. Umbilicosphaera. foliosa (ODP 1240; mcd 41.68). 5. Umbilicosphaera hulburtiana (ODP 1240; mcd 8.12). 6. Umbilicosphaera sibogae (ODP 1240; mcd 12.48). 7. Hayaster perplexus (ODP 1240; mcd 41.68). 8. Syracosphaera lamina (ODP 1240; mcd 1.53). 9. Syracosphaera pulchra (ODP 1240; mcd 41.68). 10. Pontosphaera syracusana (ODP 1241; 9.4). 11. Umbellosphaera irregularis (ODP 1241; mcd 9.4). 12. Helicosphaera carteri (ODP 1242; mcd 2.11) 


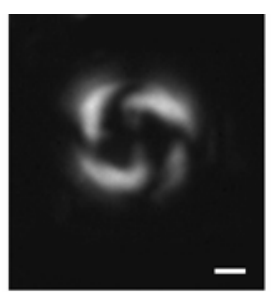

1

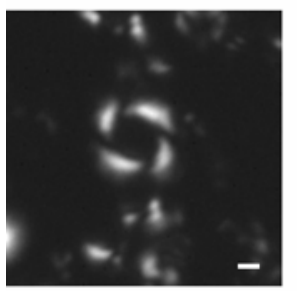

4

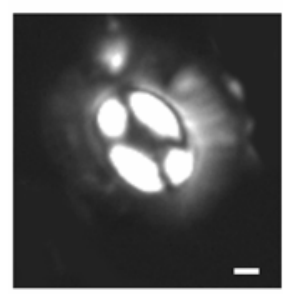

7

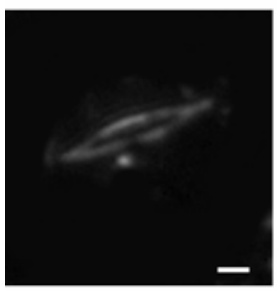

10

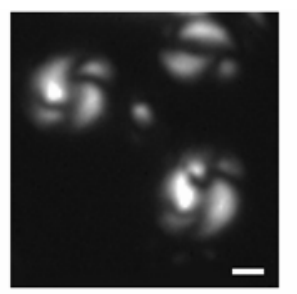

2

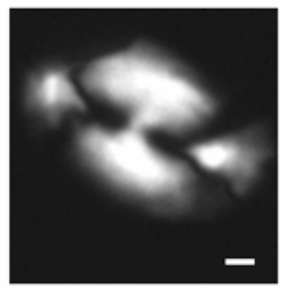

5

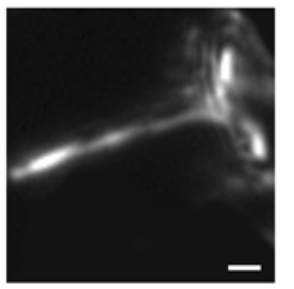

8

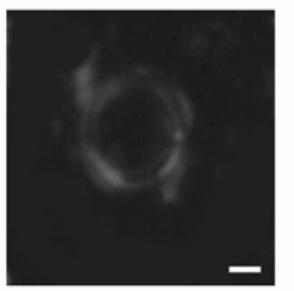

11

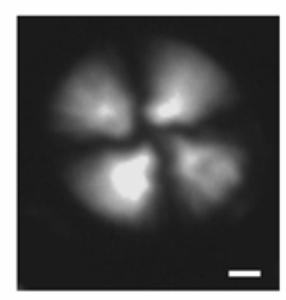

6

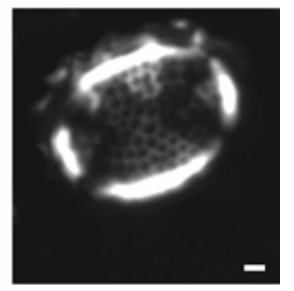

9

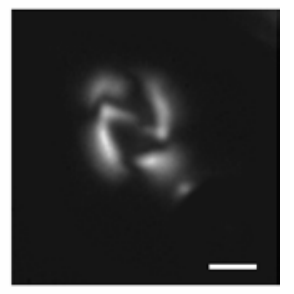

3

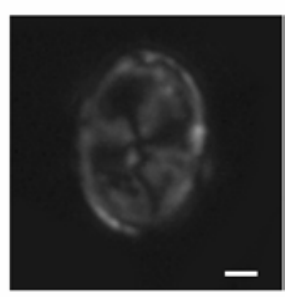

12

\section{Lámina III}

Las micrografías fueron tomadas con el microscopio óptico en el Laboratorio de Micropaleontología de la Universidad de Salamanca.

(Escala de las barras: $1 \mu \mathrm{m}$ )

1. Gephyrocapsa oceanica (ODP 1240; mcd 16.97). 2. Gephyrocapsa caribbeanica (ODP 1240; mcd 12.40). 3. Gephyrocapsa muellerae (ODP 1241; mcd 3.65). 4. Pseudoemiliania lacunosa (ODP 1240; mcd 41.68). 5. Helicosphaera carteri (ODP 1242; mcd 9.54). 6. Calcidiscus leptoporus (ODP 1240; mcd 16.97). 7. Coccolithus pelagicus (ODP 1241; mcd 12.40). 8. Rhabdosphaera clavigera (ODP 1240; mcd 4.68). 9. Pontosphaera multipora (ODP 1241; mcd 9.10). 10. Calciosolenia murrayi (ODP 1240; mcd 16.97). 11. Umbilicosphaera sibogae (ODP 1240; mcd 16.97). 12. Syracosphaera spp. (ODP 1241; mcd 2.90). 


\section{Apéndice D: Lista de acrónimos}

AABW: "Antarctic Bottom Water", Agua Antártica de Fondo

AAIW: "Antarctic Intermediate Water", Agua Intermedia Antártica, formada en el océano del Sur

ACP: "Advanced Hydraulic Piston Corer", una de las técnicas empleadas para la extracción de testigos en las campañas del ODP

AMOC: "Atlantic Meridional Overturning Circulation", Circulación Termohalina (ver THC)

ANI: "Antarctic Intermediate", término genérico para designar al agua intermedia tipo Antártico

BC: "Brazil Current", corriente del Brazil

CAR: "Carbonate Accumulation Rate", tasa de acumulación del carbonato aportado por los cocolitos, también llamado flujo del carbonato de los cocolitos

CC: "Caribe Current", corriente del Caribe (también corresponde al acrónimo de la corriente de California en los capítulos 5 y 6 , tal como ha sido utilizado en los estudios de ciencias oceanográficas)

Cca: "California Current", corriente de California (CCa, en el Capítulo 4; CC en los capítulos 5 y 6; ver CC)

CCD: "Calcite Compensation Depth", profundidad de compensación de la calcita

CPDW: "Circumpolar Deep Water", Agua Profunda Circumpolar, formada en el océano del Sur.

DIC: "Dissolved Inorganic Carbon", carbono inorgánico disuelto

ECT: "Equatorial Cold Tongue", lengua fría ecuatorial (corresponde al área de la zona de surgencia ecuatorial en el océano Pacífico oriental)

EF: "Equatorial Front", frente ecuatorial (frente convergente entre las corrientes SEC y NECC

ENSO: "El Niño Southern Oscillation", "El Niño- la Oscilación del Sur"

EUC: "Equatorial Undercurrent", corriente subsuperficial Ecuatorial

GAIW: "Gulf Alaska Intermediate Water", masa de agua intermedia formada en el golfo de Alaska

GC: "Guyana Current", Corriente de la Guayana"

GS: "Gulf Stream", corriente del Golfo

IMAGES: "Internacional Marine Past Global Change Study"

ITCZ: "Intertropical Convergence Zone", Zona de Convergencia Intertropical

JOIDES: "Joint Oceanographic Institutions for Deep Earth Sampling"

Ka: Mil años. "a" del latin "annos", corresponde al sistema internacional de unidades que se recomienda utilizar en las publicaciones de las Ciencias de la Tierra. Esta unidad fue sugerida por el Grupo IUPAC-IUGS para referir el tiempo o la edad (Rose, 2007)

Leg 202: Campaña 202 
LNADW: "Lower North Atlantic Deep Water", Agua Noratlántica Profunda inferior

LR04: "Stack" de Lisiecki y Raymo (2005)

LSW: "Labrador Sea Water", masa de agua del Labrador

Ma: Millón de años, ver Ka.

MBE: "mid-Brunhes event", evento del Brunhes medio

mbsf: "meters below sea floor", metros bajo el nivel del mar

mcd: "meters composite depth", metros de profundidad compuesta

MIS: "Marine Isotopic Stage", Estadio Isotópico Marino

MIW: "Mediterranean Intermediate Water", masa de agua intermedia del Mediterráneo

MPT: "mid-Pleistocene Transition", transición del Pleistoceno medio

MST: "Multisensor Track"

NADW: "North Atlantic Deep Water", Agua Noratlántica Profunda

NAR: "Nannofossil Accumulation Rate", tasa de acumulación de nanofósiles, también llamado flujo de cocolitos

NBC: "North Brazil Current", corriente Norte del Brazil

NPIW: "North Pacific Intermediate Water", masa de agua Intermedia del Pacífico norte

NEC: "North Equatorial Current", corriente Norecuatorial

NECC: "North Equatorial Countercurrent", contracorriente Norecuatoria

NGRIP : "North Greenland Ice Core Project"

ODP: "Ocean Drilling Program"

OIW: "Okhostk Intermediate Water", masa de agua intermedia formada en el mar de Okhotsk

OMZ: "Oxygen Minimun Zone", zona de mínimo oxígeno

PBW: "Pacific Bottom Water", masas de aguas de fondo del Pacífico

PC: "Peru Current", corriente del Perú

PICASSO: "Paleoclimatologie, Isotopes, Chimie, Atlantique, Séries Sedimentaires Océaniques"

SATFZ: "Subarctic-tropical Frontal Zone", zona del frente subártico-tropical localizado en el Pacífico Norte

SEC: "South Equatorial Current", corriente Surecuatorial

SEM: "Scanning Electron Microscope", Microscopio Electrónico de Barrido

SPECMAP: "Mapping Spectral Variability in Global Climate Project"

THC: "Thermohaline Circulation", Circulación Termohalina (ver AMOC)

UNADW: "Upper North Atlantic Deep Water", Agua Noratlántica Profunda superior

XCB: "Double Extended Core Barrel", una de las técnicas empleadas para la extracción de testigos en las campañas del ODP 